\title{
Lehrerüberzeugungen zum Lehren und Lernen
}

\author{
Dissertation \\ zur Erlangung des Doktorgrades \\ der Sozialwissenschaftlichen Fakultät \\ der Georg-August-Universität Göttingen
}

\author{
vorgelegt von \\ Natalia Schlichter \\ (geb.Tchoudova) \\ geboren in Oktjabrskij (Russland)
}

Göttingen 2012 
Erstgutachter: Prof. Dr. Rainer Watermann

Zweitgutachter: $\quad$ Prof. Dr. Matthias Nückles 


\section{Danke}

Diese Dissertation wurde durch ein Stipendium im Rahmen des Graduiertenkollegs „Passungsverhältnisse schulischen Lernens“ von der Deutschen Forschungsgemeinschaft gefördert. Für die finanzielle und materielle Unterstützung bedanke ich mich sehr.

Mein besonderer Dank gilt meinen beiden Betreuern Herrn Prof. Dr. Rainer Watermann und Herrn Prof. Dr. Matthias Nückles. Herrn Watermann danke ich insbesondere für die wertvollen methodischen und inhaltlichen Hinweise und Anregungen während der gesamten Promotionszeit. Bei Herrn Nückles bedanke ich mich für seine Expertise auf dem Themengebiet und seine inhaltlichen Anregungen.

Ebenso bedanke ich mich bei meinen Kolleginnen und Kollegen am Graduiertenkolleg für die gemeinsame Zeit in Göttingen. Danke euch allen. Besonders möchte ich mich bei Isabell Paulick für die Unterstützung und die vielen Gespräche bedanken. Mit ihrem ausgeglichenen Charakter, ihrer Loyalität und aufbauender Unterstützung leistete sie sowohl inhaltliche als auch persönliche Hilfestellung und hat stets zu mir gehalten. Herzlichen Dank!

Ein großer Dank geht auch an meine kanadischen Gastgeber Dan Pratt und John Collins für die großartigen Monate an der University of British Columbia (Vancouver, BC). Dadurch hatte ich die Möglichkeit, einen Einblick in internationale Forschung zu bekommen und mich mit ihnen und ihrem Team auszutauschen. Vielen Dank für die Gastfreudschaft und für die vielen Eindrücke. Es war eine unvergesslich schöne Zeit.

Ganz besonders danke ich meiner Mutter Rimma Alexandrowna Tschudova für die Unterstützung und Hilfe in allen Bereichen meines Lebens und meinem guten Freunden Rainer Kohl für sein Glauben an meine Fähigkeiten und sein zukunftsweisenden Blick auf meinen beruflichen und auch privaten Lebensweg. Schließlich möchte ich mich bei all meinen Freunden für die Unterstützung und Abwechslung bedanken. 
Inhalt

Verzeichnis der Tabellen ................................................................................................

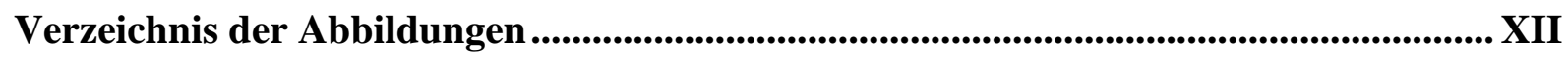

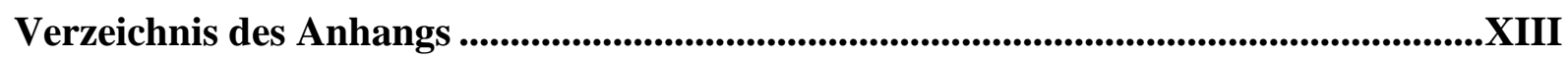

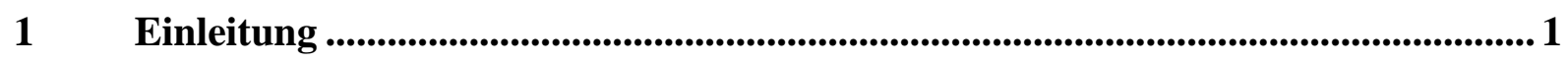

2 Theoretischer Hintergrund.......................................................................................................... 8

2.1 Lehrerüberzeugungen zum Lehren und Lernen.................................... 8

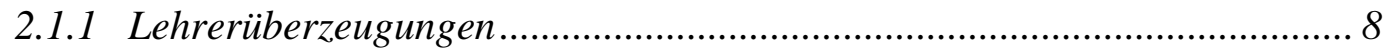

2.1.2 Lehrerüberzeugungen zum Lehren und Lernen ...................................... 9

2.1.3 Lehrerprofessionsdebatte und Lehrerüberzeugungen im Handlungskompetenzmodell der Lehrkräfte.......................................... 10

2.1.4 Lehrerüberzeugungen zum Lehren und Lernen. Eine Begriffsklärung ..... 12

2.1.5 Verschiedene Lehr-Lern-Überzeugungen............................................ 13

2.2 Überblick über den aktuellen Forschungsstand

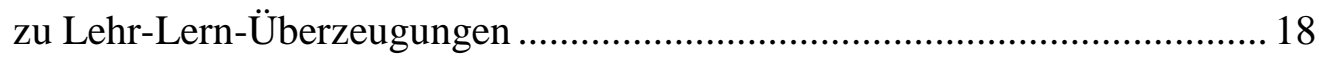

2.2.1 Entwicklung von Lehr-Lern-Überzeugungen während

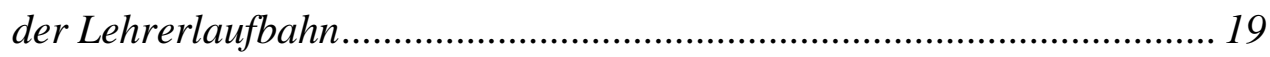

2.2.2 Einfluss der Lehr-Lern-Überzeugungen auf den Unterricht und die Schülerleistung ....................................................................... 25

2.3 Erfassung von Lehr-Lern-Überzeugungen ............................................... 29

2.3.1 Methodische Zugänge zu Lehr-Lern-Überzeugungen .............................. 29

2.3.2 Messinstrumente zur Erfassung von Lehr-Lern-Überüberzeugungen....... 30

2.3.3 Dimensionalität der Überzeugungen..................................................... 34

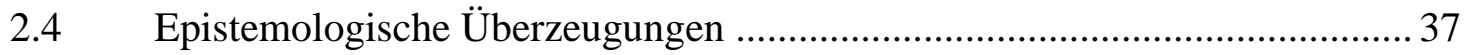

2.4.1 Epistemologische Überzeugungen der Lehrkräfte.

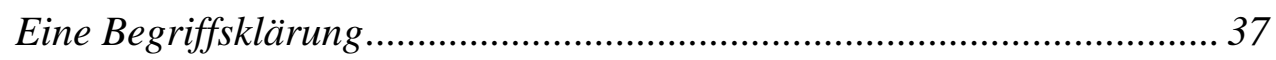

2.4.2 Modelle epistemologischer Überzeugungen.......................................... 37

2.4.3 Überblick über die Forschung zu epistemologischen Überzeugungen ..... 42

2.4.4 Messinstrumente zur Erfassung epistemologischer Überzeugungen ........ 45

2.5 Epistemologische Überzeugungen und lehr-/lernbezogene Überzeugungen . 50

3 Ableitung der Fragestellungen

3.1 Überzeugungen zum Lehren und Lernen zu unterschiedlichen Zeitpunkten in der Lehrerlaufbahn (Studie 1)

3.2 Konstruktion des Fragebogens zur Messung der vier Lehrerüberzeugungen zum Lehren und Lernen (Studie 2) 
3.3 Überprüfung des Zusammenhangs zwischen epistemologischen und den lehr/lernbezogenen Überzeugungen Transmission, Konstruktion, Partizipation und Schülerorientierung (Studie 3)

4 Teachers' beliefs about learning and teaching at different stages of teachers' career (Study 1) .........................................................................................................6 63

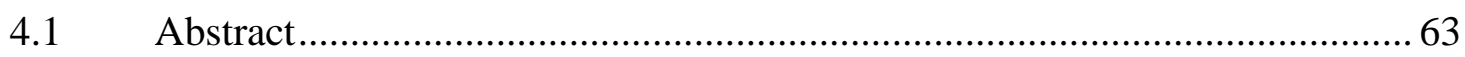

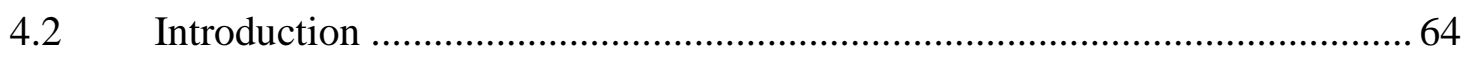

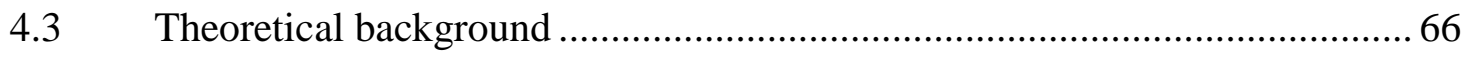

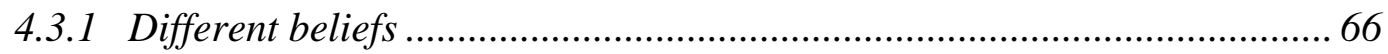

4.3.2 Variability of beliefs about learning and teaching ................................... 68

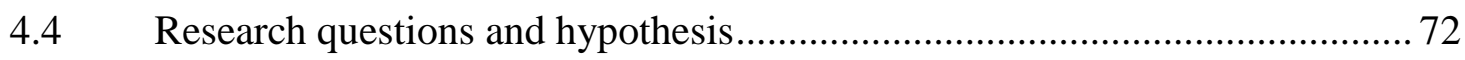

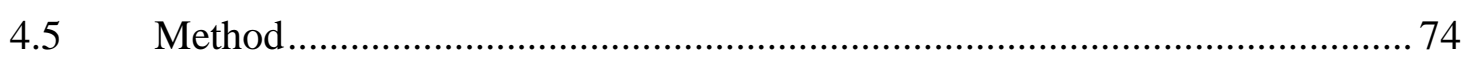

4.5.1 Participants - design ....................................................................... 74

4.5.2 Interview procedure .......................................................................... 74

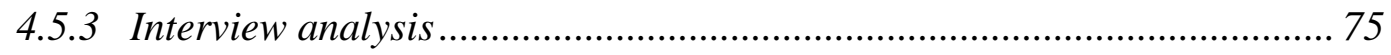

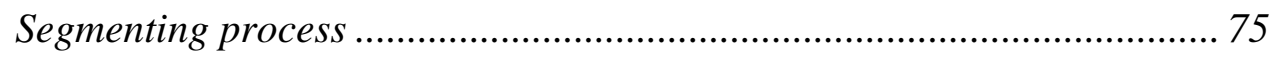

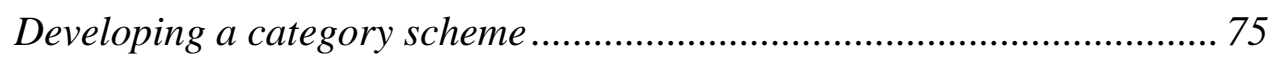

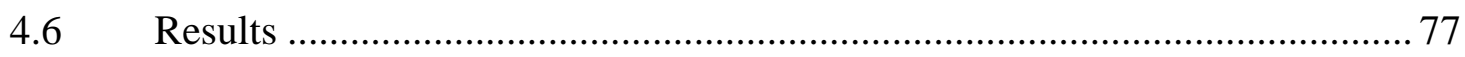

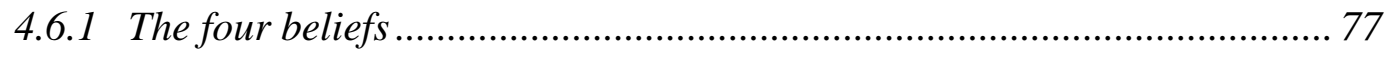

4.6.2 Differences between different stages of teacher career regarding beliefs about learning and teaching............................................................... 78

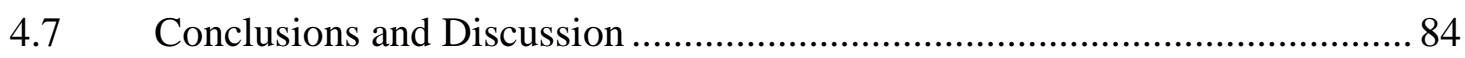

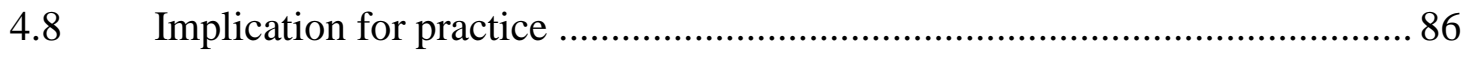

4.9 Study limitation and implication for further research................................ 87

$5 \quad$ Konstruktion und Validierung des Fragebogens zur Messung der vier Lehrerüberzeugungen zum Lehren und Lernen (Studie 2) ....................................89

5.1 Zusammenfassung und Abstract................................................................ 89

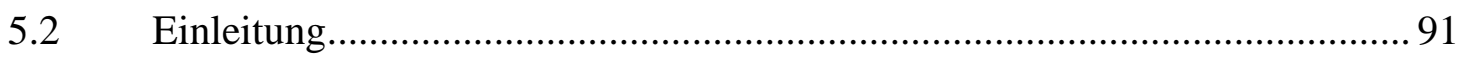

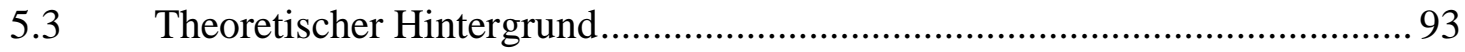

5.3.1 Lehrerüberzeugungen zum Lehren und Lernen:

Definition und Begriffsklärung der vier Überzeugungen ......................... 93

5.3.2 Stand der Forschung zur Erfassung von Überzeugungen zum Lehren und

Lernen. 94

5.3.3 Überblick über die Forschung zur Veränderung der Überzeugungen zum Lehren und Lernen................................................................................. 97

5.3.4 Epistemologische Überzeugungen und Lehr-Lern-Überzeugungen.......... 99 
5.3.5 Geschlechtsspezifität der Überzeugungen zum Lehren und Lernen........ 100

5.3.6 Fachspezifität der Überzeugungen zum Lehren und Lernen................... 100

5.4 Ableitung der Fragestellung ................................................................. 102

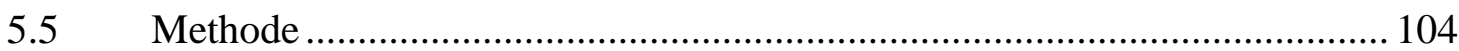

5.5.1 Skalenkonstruktion des Fragebogens zur Messung der vier

Lehrerüberzeugungen zum Lehren und Lernen

(engl. The Questionnaire of Four Teachers' Beliefs - Q4TB) ................ 104

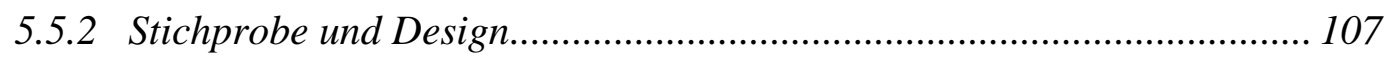

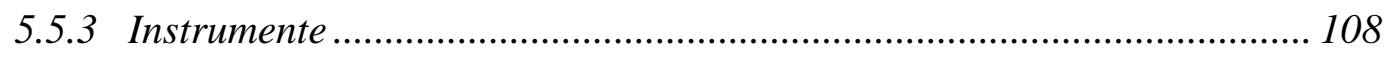

Wertehaltungen und Überzeugungen:

Lern- und Lehrbegriffsskalen, Erziehungsziele .................................... 108

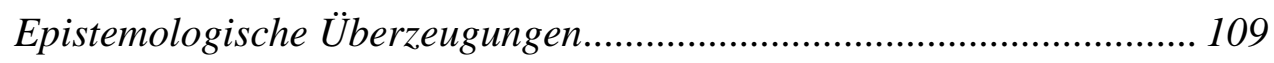

5.5.4 Umgang mit fehlenden Werten ............................................................ 109

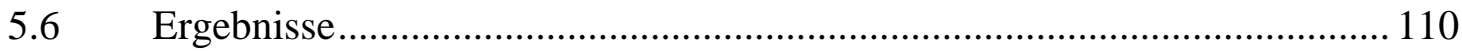

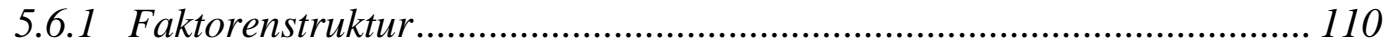

Faktorlösung ohne Vorgabe der Faktorenanzahl ................................. 110

Die 4-Faktoren-Lösung ................................................................ 115

5.6.2 Konstruktvalidierung ..................................................................... 121

Zusammenhänge zwischen den Skalen der vier Überzeugungen zum

Lehren und Lernen und den Skalen zu Lern-/Lehrbegriffen sowie

Erziehungszielen

Zusammenhänge zwischen den Skalen zu Überzeugungen zum Lehren und

Lernen und den Skalen zu epistemologischen Überzeugungen.

Geschlechts- und fächerkombinationsspezifische Unterschiede

in den Überzeugungen zum Lehren und Lernen.

Unterschiede in den Überzeugungen zum Lehren und Lernen zwischen Bachelor- und Masterstudierenden

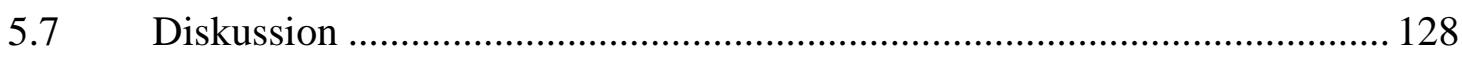

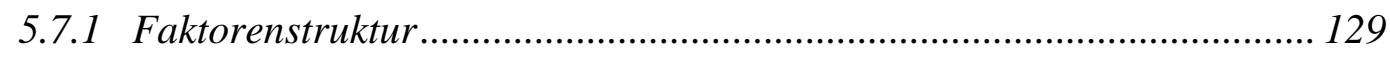

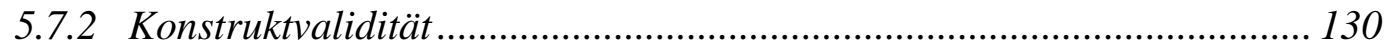

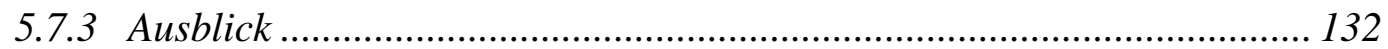

6 Überprüfung des Zusammenhangs zwischen epistemologischen Überzeugungen und den lehr-/lernbezogenen Überzeugungen Transmission, Konstruktion, Partizipation und Schülerorientierung (Studie 3) ................................................. 135

6.1 Zusammenfassung und Abstract............................................................ 135

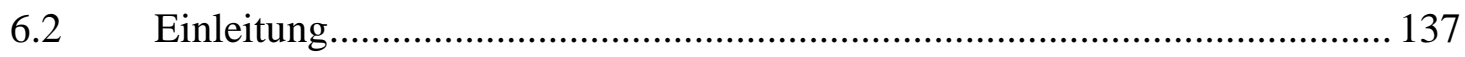


6.3 Theoretischer Hintergrund

6.3.1 Lehr-/lernbezogene Überzeugungen von Lehrkräften.

6.3.2 Überblick über die Forschung zur Veränderung von lehr-/lernbezogenen

Überzeugungen.

6.3.3 Epistemologische Überzeugungen. 143

6.3.4 Diskrepanzen in der Definition und Operationalisierung von epistemologischen und lehr-/lernbezogenen Lehrerüberzeugungen

6.3.5 Überblick über die Forschung zum Zusammenhang zwischen epistemologischen Überzeugungen und lehr-/lernbezogenen Überzeugungen. 149

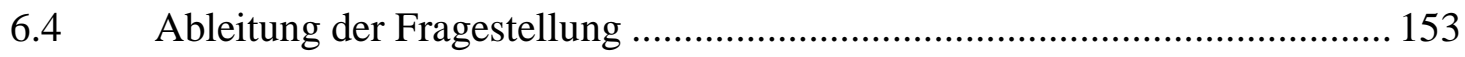

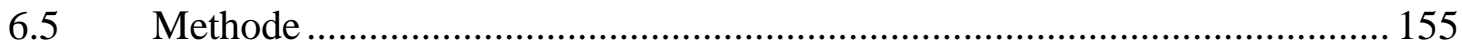

6.5.1 Stichprobe und Untersuchungsdesign ............................................... 155

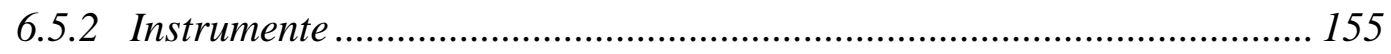

Fragebogen zur Erfassung der vier Überzeugungen zum Lehren und Lernen(engl. The Questionnaire of Four Teachers 'Beliefs - Q4TB)..... 155

Epistemologische Überzeugungen...................................................... 157

6.5.3 Umgang mit fehlenden Werten ......................................................... 159

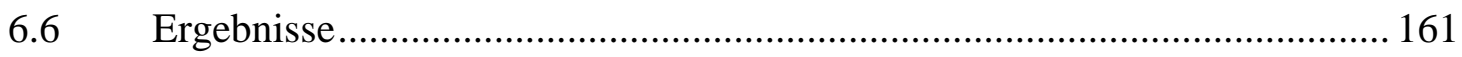

6.6.1 Zusammenhang zwischen epistemologischen Überzeugungen und den lehr/lernbezogenen Überzeugungen Transmission, Konstruktion, Partizipation und Schülerorientierung.

6.6.2 Unterschiede in den Überzeugungen zum Lehren und Lernen in Abhängigkeit vom Zeitpunkt im Studium .............................................. 162

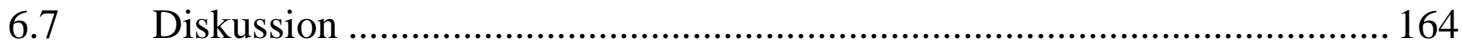

6.7.1 Fragebogen zur Messung der vier Lehrerüberzeugungen zum Lehren und Lernen. 164

6.7.2 Zusammenhang zwischen epistemologischen Überzeugungen und den lehr/lernbezogenen Überzeugungen Transmission, Konstruktion, Partizipation und Schülerorientierung 166

6.7.3 Unterschiede in den Überzeugungen zum Lehren und Lernen in Abhängigkeit vom Zeitpunkt im Studium 168

6.8 Grenzen der Studie und zukünftige Forschungsfragen................................ 169

$7 \quad$ Zusammenfassende Schlussdiskussion und Ausblick ............................................. 171

7.7 Zusammenfassung der zentralen Ergebnisse .............................................. 171

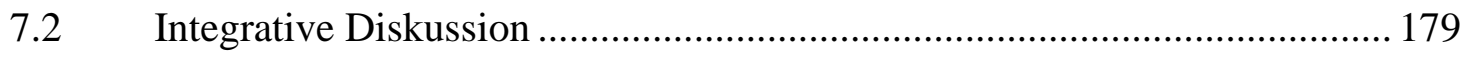

7.3 Grenzen der Arbeit und Ansätze für die weitere Forschung ........................ 186 
$8 \quad$ Zusammenfassung ............................................................................................................ 190

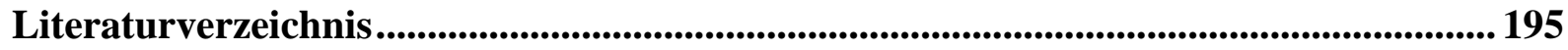

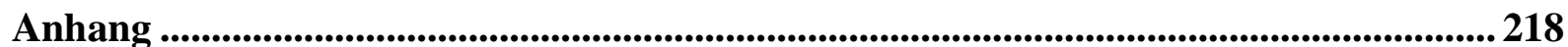




\section{Verzeichnis der Tabellen}

Tabelle 1 Beispielitem für die Itemgruppen aus dem SEQ (1990). 46

Table 2 Examples of differentiation categories and segments for each belief and for non-classified statements

Table 3 Frequencies of categories, segments and average segments in each interview for each belief . 78

Table $4 \quad$ Numbers of participants with one belief as their dominant belief. 79

Table 5 Percentages of the four beliefs (means and standard deviation) for each group and the whole sample.

Tabelle 6 Facetten der vier Überzeugungen, die Quelle der Facetten, Beispielitems sowie Anzahl der verwendeten Items (n). 106

Tabelle 7 Mittelwerte (M) und Standardabweichungen (SD) der Skalen sowie die Interkorrelationen zwischen den Skalen..... 114

Tabelle 8 Faktorladungsmatrix, gebildete Skalen (Partizipation - P; Schülerorientierung - SO; Transmission - T; Konstruktion-K), Anzahl der Items (n) und Cronbachs Alpha $(\alpha)$

Tabelle 9 Mittelwerte (M) und Standardabweichungen(SD) und Interkorrelationen der Skalen

Tabelle 10 Korrelationen zwischen den vier Skalen der Überzeugungen zum Lehren und

Lernen und den Lern- und Lehrbegriffen sowie Erziehungszielen 122

Tabelle 11 Korrelationskoeffizienten zwischen den Skalen Dualismus und Relativismus und den vier lehr-/lernbezogenen Überzeugungen - Partizipation, Schülerorientierung, Transmission und Konstruktion 124

Tabelle 12 Mittelwerte (M) und Standardabweichungen (SD) der Skalen in Abhängigkeit vom Geschlecht und von der studierten Fächerkombination

Tabelle 13 Mittelwerte (M) und Standardabweichungen (SD) der Skalen für Bachelor- und Masterstudierende 126

Tabelle 14 Skalen der lehr-/lernbezogenen Überzeugungen, Beispielitems, Anzahl der verwendeten Items (n) sowie Reliabilitäten $(\alpha)$ 156

Tabelle 15 Mittelwerte (M) und Standardabweichungen (SD) und Interkorrelationen der Skalen

Tabelle 16 Skalen der epistemologischen Überzeugungen, Beispielitems, Anzahl der verwendeten Items $(\mathrm{n})$ sowie Reliabilitäten $(\alpha)$ 
Tabelle 17 Korrelationskoeffizienten der Überzeugungen zum Lehren und Lernen und den Skalen sowie der Gesamtskala zu epistemologischen Überzeugungen.......... 161

Tabelle 18 Mittelwerte (M) und Standardabweichungen (SD) der vier Überzeugungen für die drei Gruppen: Studierenden am Anfang, in der Mitte und am Ende des Studiums 


\section{Verzeichnis der Abbildungen}

Abbildung 1 Das professionelle Handlungskompetenzmodell von Lehrkräften von COACTIV (Kunter et al., 2011, S. 32).

Figure 2 Counts (means) of the segments regarding learning and teaching for the five

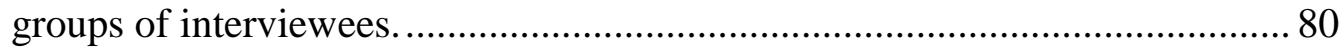

Figure 3 Distribution of the percentages of the beliefs for the five groups of the interviewees. 


\section{Verzeichnis des Anhangs}

Anhang A Überblick über die verschiedenen Überzeugungen in ihrer Relation zueinander.

Anhang B Mittelwerte (M) und Standardabweichungen (SD) der Items der 14-Faktoren-

Lösung aus der Studie 2

Anhang C Faktorladungsmatrix, gebildete Skalen (Partizipation - P; Schülerorientierung

- SO; Transmission - T; Konstruktion-K), Anzahl der Items (n) und Cronbachs Alpha $(\alpha)$ aus der Studie 3 225

Anhang D Mittelwerte (M) und Standardabweichungen (SD) der Items aus der Studie 3 





\section{$1 \quad$ Einleitung}

Etwa seit Beginn der 1980er Jahre beschäftigt sich die Lehrerforschung mit der Frage, welche Einstellungen und Überzeugungen Lehrkräfte zum Lehren und Lernen haben und in welchem Zusammenhang diese mit ihrem unterrichtlichen Handeln stehen (z.B. Clark, 1988; Cole, 1989; Fenstermacher, 1979; Nespor, 1987; Pintrich, 1990). Während das sogenannte Prozess-Produkt-Paradigma (Bromme \& Rheinberg, 2006) einzelne Verhaltensmuster der Lehrkräfte mit ihrer Wirkung auf das schulische Lernen analysierte, folgt die zeitgenössische empirische Forschung eher einem Expertenmodell, das die Bedeutung verschiedener Facetten der Lehrerkompetenz - wie etwa das Professionswissen und die Lehrerüberzeugungen - in den Mittelpunkt rückt (Baumert \& Kunter, 2006; Bromme \& Rheinberg, 2006). Dieser Paradigmenwechsel beruht auf der generellen Annahme, die Entscheidungen, die Personen in ihrem täglichen Leben fällen, ausgehend von deren Überzeugungen, vorhersagen und so ihr Handeln erklären zu können (Bandura, 1986; Dewey, 1933).

In Bezug auf lehr-/lernbezogene Überzeugungen werden in der jüngsten quantitativen Forschung meist knowledge transmission (dt. Transmission) und cognitive construction (dt. Konstruktion) unterschieden (z.B. Kunter, Baumert, Blum, Klusmann, Krauss \& Neubrand, 2011; OECD, 2009; Peterson, Fennema, Carpenter, \& Loef, 1989; Staub \& Stern, 2002). Dabei wird das Lernen nach der transmissiven Überzeugung als Aufnahme von objektiv gegebenen Wissenseinheiten verstanden sowie das Lehren als ein einfacher Transfer dieses Wissens. Nach der konstruktivistischen Vorstellung wird das Lernen als eine aktive und eigenständige Wissenskonstruktion aufgefasst und das Lehren als eine Unterstützung dieser Konstruktionsprozesse (z.B. Bereiter, 1994; Dubberke, Kunter, McElvany, Brunner \& Baumert, 2008; Kunter et al., 2011; Pajares, 1992; Peterson et al., 1989; Staub \& Stern, 2002). Seidel, Schwindt, Rimmele und Prenzel (2008) sprechen in diesem Kontext von rezeptiven und konstruktivistischen, Chan und Elliott (2004) von traditionellen und konstruktivistischen und Handal (2003) von traditionellen und progressiven Überzeugungen (vgl. auch Meirink, Meijer, Verloop \& Bergen, 2009). Somit wird die transmissive Überzeugung, auch rezeptiv genannt, als die mit der längeren Tradition bzw. als die ältere der beiden präsentiert. Dagegen basiert die konstruktivistische Überzeugung auf einer jüngeren Lerntheorie, welche das Lernen als einen aktiven Prozess definiert. Solch eine Gegenüberstellung der beiden Überzeugungen birgt leicht die Gefahr, vergleichend interpretieren zu wollen. Dagegen weist zum Beispiel Sfard (1998) in ihren theoretischen Ausführungen über Metaphern des Lehrens und Lernens auf die Gefahr hin, eine der beiden Vorstellungen vom Lehren und Lernen zu 
bevorzugen. Ausgehend von der Kritik der subjektiv gegebenen Objektivität des Wissens beschreibt Sfard (1998) eine partizipatorische Vorstellung vom Lehren und Lernen (engl. participation). Anders als in der konstruktivistischen Perspektive als Eigenkonstruktion oder in der transmissiven Vorstellung als persönlicher Besitz wird das Wissen in der partizipatorischen Vorstellung als ein in der Gesellschaft geteiltes Gut verstanden. Diese Vorstellung basiert auf dem Lerngemeinschaftsansatz (engl. learning as participation in a learning community) (Lave \& Wenger, 1991; Sfard, 1998). In der empirischen Forschung wurde diese Vorstellung als teaching as engaging in community (Alger, 2009) und als situative/sociohistoric view (Martínez, Sauleda \& Huber, 2001) untersucht. In der partizipatorischen Sicht wird das Lernen als Resultat einer authentischen Teilhabe an den Aktivitäten einer Lerngemeinschaft verstanden, die das Wissen durch die ständige Zusammenarbeit gemeinsam aushandelt. Ergebnisse dieser qualitativen Studien zeigen, dass gerade unter Lehrkräften, die entweder in der Ausbildung sind oder über erste, jedoch wenige Berufserfahrungen verfügen, partizipatorische Überzeugungen überwiegen (Alger, 2009; Martínez et al, 2001).

Pratt (2002), der ähnlich wie Sfard (1998) eine pluralistische Position in Bezug auf Lehrerüberzeugungen vertritt, beschreibt neben den drei genannten Überzeugungen die Perspektive nurturing (dt. Schülerorientierung ${ }^{1}$ ). Diese bezieht sich auf das Lehrer-SchülerVerhältnis (siehe auch student-teacher-interaction bei Kember, 1997; establishing interpersonal relationship bei Dunkin \& Precians, 1992). Die inhaltliche Ausrichtung der Perspektive auf die Schülerpersönlichkeit spiegelt motivationstheoretische Lernansätze (Deci \& Ryan, 1987, 1993) sowie das Konstrukt des unterstützenden Lernklimas wider (Klieme, Lipowsky, Rakoczy \& Ratzka, 2006). Ähnliche Aspekte finden sich auch bei den professionellen Lehrerstandards nach Oser (1998, 2009). So wird beispielsweise bei der Berufsethik - Fürsorge und Gerechtigkeit - explizit der Umgang der Lehrkraft mit den Schülerinnen und Schülern thematisiert und die Wichtigkeit der Lehrer-Schüler-Beziehung für den Lehrerberuf unterstrichen. In der großen Meta-Analyse von Hattie (2009) mit 50000 Studien wurde gezeigt, dass im Vergleich zur direkten Instruktion $(d=.59)$, zum kooperativen ( $d=.41)$ oder zum entdeckenden Lernen $(d=.31)$ das Lehrer-Schüler-Verhältnis einen besonders wichtigen Faktor für das erfolgreiche Lernen $(d=.72)$ darstellt. Dieses Ergebnis stützt die meisten Studien zu Überzeugungen von Lehramtsstudierenden, welche zeigten, dass die Lehrer-Schüler-Beziehung für angehende Lehrkräfte von großer Bedeutung ist (z.B.

\footnotetext{
1 Der Begriff „Schülerorientierung“ wird als deutsche Entsprechung des Begriffes „nurturing“ verwendet. Siehe dazu Kapitel 2.1.5.
} 
Brookhart \& Freeman, 1992; Rathbone \& Pierce, 1990; Weinstein, 1989; Wideen, MayerSmith \& Moon, 1998).

Insgesamt wird den Lehrerüberzeugungen sowohl in theoretischen als auch in empirischen Arbeiten eine Bedeutung für das unterrichtliche Handeln und für Unterschiede im Instruktionsverhalten zugeschrieben (vgl. Fennema, Carpenter \& Loef, 1990; Fischler, 2000; Köller, Baumert \& Neubrand, 2000; Schönfeld, 1983, 2000; Stipek, Givvin, Salmon \& MAcGyvers, 2001). Empirisch wurde in einigen Studien der Einfluss der lehr-/lernbezogenen Überzeugungen auf das Lehrerhandeln, die Schülerwahrnehmung des Unterrichts und die Schülerleistung demonstriert (vgl. Brunner et al., 2006; Hartinger, Kleickmann \& Hawelka, 2006; Peterson et al., 1989; Staub \& Stern, 2002). Lehrkräfte mit weniger transmissiven Überzeugungen sorgten zum Beispiel für ein höheres Ausmaß an kognitiver Aktivierung im Unterricht und gaben den Schülerinnen und Schülern häufiger konstruktive Unterstützung als Lehrkräfte mit stärker transmissiven Überzeugungen (Dubberke et al., 2008), was sich wiederum positiv auf die Schülerleistungen in Mathematik auswirkte (Dubberke et al., 2008; Staub \& Stern 2002). Gerade in den jüngeren Untersuchungen konnte dieser Effekt auch unter Kontrolle konkurrierender Lehrer- und Schülermerkmale (z.B. Berufserfahrung und fachdidaktisches Wissen bei Lehrkräften, Vorwissen und Geschlecht sowie Jahrgangstufe bei Schülerinnen und Schülern) nachgewiesen werden (Dubberke et al., 2008; Hartinger et al., 2006). Somit deuteten einige Befunde der jüngeren Lehrerforschung darauf hin, dass es tatsächlich eine Verbindung zwischen Lehr-Lern-Überzeugungen von Lehrkräften, deren Unterrichtsgestaltung und dem Lernen der Schülerinnen und Schüler gibt. Aus diesem Grund sind Lehrerüberzeugungen für die Lehrerforschung und -ausbildung von Bedeutung (Hartinger et al., 2006; Richardson, 1996). Besteht Kenntnis über deren Funktionsweisen, kann eine Verbesserung der unterrichtlichen Praxis und eine Unterstützung der angehenden Lehrkräfte bei der Unterrichtsplanung und -gestaltung bewirkt werden (z.B. Ashton, 1990; Brookhart \& Freeman, 1992; Richardson, 1996). Bei der Annahme des Zusammenhangs zwischen Lehrerüberzeugungen und dem Verhalten der Lehrkräfte ließe sich auch vermuten, dass sich durch eine Veränderung der Überzeugungen eine gezielte Veränderung des Handelns erreichen ließe. Dies wäre ein ökonomischer Weg zur Intervention des Lehrerhandelns.

Seit den ersten Arbeiten zu Lehrerüberzeugungen (Berliner, 1988; Borko \& Livington, 1989; Calderhead, 1981; Cooney, 1985; Dann, Müller-Fohrbrodt \& Cloetta, 1981; Hollingsworth, 1989; Munby, 1987; Nespor, 1987) steht die Frage nach deren Entwicklung im Mittelpunkt der Aufmerksamkeit. Die empirische Forschung zu dieser Frage lieferte 
einerseits zahlreiche Hinweise auf die sich mit steigenden akademischen und praktischen Berufserfahrungen verändernden Lehrerüberzeugungen (Alger, 2009; Leavy, McSorley \& Bote, 2007; Martínez et al., 2001). Andererseits wurde das Ausbleiben von Veränderungen festgestellt (Tilemma \& Knoll, 1997; Weinstein, 1990). Diese Diskrepanzen der Ergebnisse können durch die unterschiedliche Operationalisierung der Überzeugungen erklärt werden. Zum Teil werden Überzeugungen mithilfe eines kurzen Textes, der metaphorisch eine Überzeugung darstellt, präsentiert (Alger, 2009), zum Teil werden in Interviews Einzelaspekte, beispielsweise „der gute Lehrer“, erfragt (Ng, Nicholas \& Williams, 2010). Zudem sind die meisten Studien qualitativ und beziehen sich auf unterschiedliche theoretische Hintergründe. Daher können die bisherigen empirischen Ergebnisse wenig aufeinander bezogen werden und führen nicht zu eindeutigen Erkenntnissen. Weiterhin beschränken sich existierende Querschnittstudien auf Vergleiche von meist nur zwei Zeitpunkten in der Lehrerlaufbahn, zum Beispiel auf den Anfang und das Ende der universitären Lehrerausbildung (z.B. Nettle, 1998) oder die Zeit vor und während der Schulpraxis (z.B. Martínez et al, 2001). Längsschnittstudien wurden bislang kaum durchgeführt. Einen Versuch in diese Richtung stellt die retrospektive Befragung von Lehrkräften (Alger, 2009) darüber dar, wie sie ihre Überzeugungen zu der Zeit, als sie noch am Anfang ihrer beruflichen Karriere standen, einschätzten. Insgesamt scheint es jedoch notwendig, die Lehr-LernÜberzeugungen der Lehrkräfte zu mehreren Zeitpunkten ihrer beruflichen Laufbahn zu untersuchen, vor allem, wenn man Aussagen über zwei Zeitpunkte hinaus treffen möchte. Eine solche Untersuchung würde nicht nur die Frage nach der Entwicklung bzw. Veränderung von Überzeugungen zum Lehren und Lernen beantworten, sondern andere, beispielsweise partizipatorische und schülerorientierte Lehr-Lern-Überzeugungen mit deren Bedeutung zu unterschiedlichen Zeitpunkten der Lehrerlaufbahn aufzeigen.

Neben den Überzeugungen zum Lehren und Lernen wird in der Lehr-Lern-Forschung auch die Bedeutung epistemologischer Überzeugungen in Lernkontexten diskutiert. Epistemologische Überzeugungen beinhalten Auffassungen über die Natur von Wissen, über dessen Struktur und Genese. In Bezug auf epistemologische Überzeugungen wird ebenfalls angenommen, dass sie die Art der Begegnung mit der Welt vorstrukturieren (Duell \& Schommer-Aikins, 2001; Hofer, 2000, 2001; Hofer \& Pintrich, 1997; Howard, McGee, Schwartz \& Purcell, 2000; Schommer, 1990, 1994b). Verglichen mit der Forschung zu LehrLern-Überzeugungen hat die Forschung zu epistemologischen Überzeugungen eine lange Tradition und weist einige gut evaluierte Messinstrumente vor (Schommer, 1990; Hofer \& Pintrich, 2002; Pajares, 1992). Allerdings wurde dabei das Hauptaugenmerk auf die 
epistemologischen Überzeugungen von Schülerinnen und Schülern gerichtet und deren Einfluss beispielsweise auf das Lernen untersucht (Baumert et al., 2000; Trautwein \& Lüdtke, 2004). Insgesamt überrascht das geringe Interesse an den epistemologischen Überzeugungen von Lehrkräften (Maggioni \& Parkinson, 2008). Epistemologische Überzeugungen wurden oft als Teil von lehr-/lernbezogenen Überzeugungen betrachtet (Dubberke et al., 2008). Dahinter steht offenbar die Annahme, dass eine bestimmte Vorstellung von Wissen eine bestimmte Vorstellung von Lernen mit sich bringt. Zum Beispiel hieß es, dass Lehrkräfte, die Wissen als einfach und gesichert auffassen, eine rezeptive Überzeugung zum Lernen besitzen und Lehrkräfte, die das Wissen als ein Netzwerk von Theorien verstehen, der Überzeugung sind, dass Schülerinnen und Schüler ihr Wissen durch Verständigungs- und Aushandlungsprozesse selbst konstruieren (Olafson \& Schraw, 2006). Während einige empirische Studien diese Annahme belegen konnten (Chen \& Elliott, 2004; Seidel et al., 2008), lieferten andere gegensätzliche Hinweise. So demonstrierten Cheng, Chan, Tang und Cheng (2009) in ihrer Studie, dass fortgeschrittene epistemologische Überzeugungen entgegen den Erwartungen mit traditionellen Lehr-Lern-Vorstellungen zusammenhängen können. Somit scheint die Frage nach dem Zusammenhang zwischen epistemologischen Überzeugungen und lehr-/lernbezogenen Überzeugungen von der empirischen Forschung bislang nicht hinreichend beantwortet. Vor allem in Bezug auf die bisher wenig untersuchten partizipatorischen und schülerorientierten Lehr-Lern-Überzeugungen besteht weiterer Forschungsbedarf.

Hinsichtlich der lehr-/lernbezogenen Überzeugungen ist der bestehende Forschungsbedarf darauf zurückzuführen, dass sich die bisherige empirische Forschung auf das Überzeugungspaar knowledge transmission vs. cognitive construction konzentrierte (Dubberke et al., 2008; Fennema et al., 1990; OECD; 2009; Staub \& Stern, 2002). Sowohl theoretische als auch empirische Hinweise auf die Notwendigkeit der Ausweitung des Konstrukts auf andere Überzeugungen wurden bislang weitestgehend ignoriert. Mit Ausnahme vereinzelter Studien (Alger, 2009; Martínez et al., 2001) fanden partizipatorische und schülerorientierte Vorstellungen zum Lehren und Lernen in der empirischen Forschung zu Lehrerüberzeugungen kaum Beachtung, obwohl diese offenbar von besonderer Wichtigkeit für angehende Lehrkräfte sind (Brookhart \& Freeman, 1992; Hollingsworth, 1989; Wan Ng, Nicholas \& Williams, 2010). Der pluralistischen Position von Pratt (2002) folgend, der sich gegen einen „konstruktivistischen Dogmatismus“ ausspricht, wird in der vorliegenden Arbeit von einer Komplexität der Lehrerüberzeugungen ausgegangen: Es wird angenommen, dass 
mehr als die zwei Überzeugungen knowledge transmission und cognitive construction in den Lehr-Lern-Vorstellungen der Lehrkräfte existieren.

Das zentrale Anliegen dieser Arbeit wird demnach wie folgt definiert: Über die beiden Überzeugungen - Transmission und Konstruktion - hinaus sollen weitere Überzeugungen zum Lehren und Lernen untersucht werden. In der vorliegenden Arbeit wird der Frage nachgegangen, inwieweit sich diese verschiedenen Überzeugungen in den Interviews mit (angehenden) Lehrkräften identifizieren und mithilfe eines quantitativen Messinstruments erfassen lassen. Das Hauptziel der Dissertation liegt somit in der empirischen Erfassung weiterer Überzeugungen zum Lehren und Lernen. Zugleich wird in den Analysen der Fokus darauf gelegt, welche Bedeutung den verschiedenen Überzeugungen zu unterschiedlichen Zeitpunkten in der Lehrerlaufbahn zukommt. Es soll analysiert werden, in welcher Konstellation sich die verschiedenen Überzeugungen im Verlauf der Lehrerausbildung und im Lehrerberuf identifizieren lassen. Mit dieser Fragestellung wird versucht, Hinweise auf die Entwicklung bzw. die Veränderung der Überzeugungen zu erzielen, ohne eine aufwendige Längsschnittstudie durchzuführen. Dafür werden im Rahmen der ersten Interviewstudie (Studie 1) Lehramtsstudierende zu unterschiedlichen Zeitpunkten ihres Studiums, Referendare und erfahrene Lehrkräfte zu ihren Überzeugungen zum Lehren und Lernen befragt. Auf der Basis der Interviewergebnisse sowie in Anlehnung an entsprechende Lehr-Lern-Theorien und existierende Instrumente wird der Versuch unternommen, die identifizierten Überzeugungen mithilfe eines Fragebogens zu erfassen. Der so entwickelte Fragebogen wird dann in der zweiten Studie mit Studierenden des Lehramtes eingesetzt und evaluiert (Studie 2). Dabei stehen die Fragen im Fokus, inwieweit die in den Interviews und der Theorie identifizierten Dimensionen lehr-/lernbezogener Überzeugungen auch in einem quantitativen Zugriff identifiziert und empirisch voneinander getrennt werden können und, ob sich Unterschiede in den Überzeugungen je nach dem Zeitpunkt im Studium zeigen. In einer weiteren Befragung mit Studierenden (Studie 3) soll die Frage nach dem Zusammenhang zwischen verschiedenen lehr-/lernbezogenen und epistemologischen Überzeugungen analysiert werden.

In Kapitel 2 der vorliegenden Arbeit wird der theoretische Rahmen vorgestellt: Hier werden zuerst die Begriffe „Lehrerüberzeugungen“ und „Lehrerüberzeugungen zum Lehren und Lernen“ erläutert und deren Einbettung in das Modell professioneller Handlungskompetenzen der Lehrkräfte dargestellt (Baumert \& Kunter, 2006). Es werden die aus der Forschung bekannten Lehr-Lern-Überzeugungen und deren Beziehung zueinander skizziert. Des Weiteren wird ein Überblick über die Forschung zur Entwicklung bzw. Veränderbarkeit von Lehr-Lern-Überzeugungen sowie zum Einfluss der Lehr-Lern- 
Überzeugungen auf den Unterricht und die Schülerleistung gegeben. Zudem werden die methodischen Zugangsmöglichkeiten erörtert sowie existierende Instrumente zur Erfassung von Lehrerüberzeugungen zum Lehren und Lernen präsentiert. Weiterhin wird der Begriff „epistemologische Überzeugungen“ genauer beleuchtet: Es werden eine Definition des Begriffs sowie Modelle epistemologischer Überzeugungen vorgestellt, ein Überblick über die Forschung zu epistemologischen Überzeugungen gegeben sowie Instrumente zur Messung dieser präsentiert. Zudem wird ein Überblick über die Forschung zum Zusammenhang zwischen epistemologischen Überzeugungen und lehr-/lernbezogenen Überzeugungen dargestellt. Daraus werden in Kapitel 3 die Fragestellungen abgeleitet.

Die darauf folgenden Kapitel 4, 5 und 6 beinhalten die drei empirischen Studien dieser Arbeit.

Kapitel 4 beschreibt die Studie 1, in deren Rahmen Interviews mit Lehramtsstudierenden und Lehrkräften zu ihren Überzeugungen zum Lehren und Lernen durchgeführt werden.

Kapitel 5 stellt die Studie 2 dar, in welcher die Reliabilität und die Konstruktvalidität des neu entwickelten Fragebogens sowie geschlechts- und fachkombinationsspezifische Unterschiede in den Lehr-Lern-Überzeugungen untersucht werden sollen. Zudem sollen Überzeugungen von Bachelor- und Masterstudierenden miteinander verglichen werden, um deren Bedeutung in Abhängigkeit vom Zeitpunkt im Studium zu analysieren.

In Kapitel 6 wird die dritte empirische Studie vorgestellt, in der der Zusammenhang zwischen epistemologischen und lehr-/lernbezogenen Überzeugungen untersucht werden soll.

Diese drei Studien stellen den Kern der vorliegenden Arbeit dar und werden so verfasst, dass sie in Fachzeitschriften zur Veröffentlichung eingereicht werden können. Daher soll Kapitel 4 (Studie 1) auf Englisch verfasst werden, um einen Beitrag bei der Zeitschrift Teaching and Teacher Education einzureichen. Kapitel 5 und 6 (Studien 2 und 3) wurden auf Deutsch verfasst, zur Einreichung bei deutschsprachigen Fachzeitschriften.

In Kapitel 7 werden die Ergebnisse der Studien noch einmal zusammengefasst und diskutiert. Dabei werden weitergehende methodische Überlegungen erörtert sowie Implikationen für die Lehrerbildung dargestellt. Die Arbeit schließt mit einem Ausblick auf zukünftige Forschungsfragen ab. 


\section{Theoretischer Hintergrund}

\subsection{Lehrerüberzeugungen zum Lehren und Lernen}

Im Folgenden wird der zentrale Begriff der Arbeit definiert. Es wird zuerst allgemein auf Lehrerüberzeugungen und dann eingrenzend auf Lehrerüberzeugungen zum Lehren und Lernen eingegangen. Es wird zudem die Lehrerprofessionsdebatte kurz umrissen, die für das Thema der Arbeit von besonderer Wichtigkeit ist. Anschließend wird die Definition des Begriffs präsentiert sowie ein Überblick über die verschiedenen Lehr-Lern-Überzeugungen gegeben.

\subsubsection{Lehrerüberzeugungen}

Definitionen von Lehrerüberzeugungen wurden oft in Abgrenzung zu Definitionen von Wissen vorgenommen (Abelson, 1979; Nespor, 1987). Dabei wurden Überzeugungen allgemein als auf Bewertung oder Beurteilung basierend definiert und Wissen mit Fakten gleichgesetzt. In seinem Überblicksartikel zu Lehrerüberzeugungen beschrieb Pajares (1992) die beiden Begriffe als untrennbar. Ebenfalls wurden Überzeugungen und Wissen nach Verloop, van Driel und Meijer (2001) als ,an overarching, inclusive concept, summarizing a large variety of cognition, from conscious and well-balanced opinions to unconscious und unreflected intuitions" bezeichnet (S. 446). In neueren Arbeiten wird die Trennung beibehalten, wobei aufgrund fließender Übergänge zwischen den beiden Begriffen eine klare Unterscheidung nicht ohne weiteres zu vollziehen ist (Borko \& Putnam, 1996). Gleichwohl beanspruchen Wissen und Überzeugungen jeweils einen unterschiedlichen epistemologischen Status. Im Gegensatz zum Wissen verlangen Überzeugungen keine Rechtfertigung oder Widerspruchsfreiheit (Fenstermacher, 1994) und sind evaluativ (Nisbett \& Ross, 1980) sowie affektiv (Nespor, 1987). Sie werden als mehr oder weniger klare Annahmen über Charakteristika von Objekten (Nisbett \& Ross, 1980) oder als mentale Repräsentationen gesammelter Erfahrungen verstanden (Sigel, 19985). Harvey (1986) definiert Überzeugungen als persönliche Repräsentationen der Realität, welche von einer Person für gültig, wahr und glaubwürdig gehalten werden, sodass diese dementsprechend denkt und handelt. Überzeugungen beeinflussen die Interpretationen konkreter Situationen (Calderhead \& Robson, 1991). Nach Brown und Cooney (1982) sind Überzeugungen Dispositionen, die das Handeln am meisten bestimmen. Martínez et al. (2001) verwenden die Bezeichnung 
„,blueprint of thinking“ (S. 965), um den wenig sichtbaren, aber immer währenden Einfluss der Überzeugungen auf das Handeln zu demonstrieren. Auch im COACTIV-Projekt (Kognitiv Aktivierender Mathematikunterricht) (z.B. Kunter et al., 2011), deren Studien für die vorliegende Arbeit in ihrer Relevanz insgesamt hervorzuheben sind, werden handlungssteuernde Eigenschaften von Lehrerüberzeugungen thematisiert. Zusammenfassend lässt sich festhalten, dass in allen theoretischen Arbeiten Konsens besteht, dass Lehrerüberzeugungen sowohl die Wahrnehmung der Umwelt als auch das Handeln der Lehrkräfte beeinflussen (z.B. Kunter et al., 2011).

\subsubsection{Lehrerüberzeugungen zum Lehren und Lernen}

Trotz vieler Bemühungen, den Begriff Lehrerüberzeugungen zum Lehren und Lernen (engl. teachers' beliefs about teaching and learning) zu definieren, bleibt es ein „messy construct“ (Pajares, 1992, S. 307). Allein begrifflich findet sich in der Literatur eine beträchtliche Menge an Parallelbezeichnungen: Überzeugungen (engl. beliefs) (z.B. Aguirre $\&$ Speer, 2000; Peterson et al., 1989), Konzeptionen (engl. conceptions) (z.B. Boulton-Lewis, Smith, McCrindle, Burnett \& Campbell, 2001; Cheng et al., 2009; Kember, 1997; Martin \& Balla, 1991), subjektive Theorien (engl. personal theories) (Levin \& He, 2008), Überzeugungen und Absichten (engl. beliefs and intentions) (Norton, Richardson, Hartley, Newstead \& Mayes, 2005), dominierende und rezessive Perspektiven (engl. dominant and recessive perspectives) bestehend aus Handlungen, Absichten und Überzeugungen (engl. actions, intentions and beliefs) (Pratt, 2005), Lehransätze (approaches to teaching), bestehend aus Lehrstrategien und Absichten (engl. teaching strategies and intentions) (Trigwell \& Prosser, 2004), Auffassungen über Lehren und Lernen (engl. view of learning and teaching) (z.B. Dahlgren \& Chiriac, 2009), fachdidaktische Überzeugungen (engl. pedagogical content beliefs) (Staub \& Stern, 2002) und schließlich konzeptuelle Metaphern (engl. conceptual metaphors), wie diese bei Lakoff und Johnson (1980) definiert werden (Alger, 2009; Martínez et al., 2001; Saban, 2010; Sfard, 1998). Im deutschsprachigen Raum finden sich die Begriffe Überzeugungen zum und Vorstellungen über Lehren und Lernen, die weitgehend synonym zu verstehen sind (Überzeugungen bei Dubberke et al., 2008; Seidel et al., 2008; und Lehrervorstellungen bei Hartinger et al., 2006). Es kann festgehalten werden, dass innerhalb der Forschung zu Lehrerüberzeugungen viele Bezeichnungen verwendet wurden, die alle ähnliche Inhalte beschreiben, allerdings jene Sichtweise in den Vordergrund stellen, die nach Meinung der Autoren am besten den Zugang zu Lehrerkognitionen erlaubt. So sind es bei 
Trigwell und Prosser (2004) „Lehransätze mit Strategien und Absichten“ und bei Pratt (2005) „dominierende und rezessive Lehrperspektiven“. Dennoch, so die Feststellung von Speer (2005), ist den unterschiedlichen Bezeichnungen gemein, dass sie alle Lehrerkognitionen bezeichnen. Ähnlich der Feststellung von Kember, dass „,the meaning [of belief] seems to be largely synonymous with the [...] definition of conceptions" (Kember, 1997, S. 257), werden in der vorliegenden Arbeit die unterschiedlichen Bezeichnungen synonym verstanden und die entsprechenden Studien als theoretischer Hintergrund der eigenen, nachfolgend präsentierten Studien behandelt.

Für die Eingrenzung des Begriffs wurden mögliche Objekte der Überzeugungen verwendet. So können Lehrereinstellungen zu (Aus-) Bildung, zum Lehren, Lernen und zu Lernenden (Rokeach, 1968) ausgemacht werden, zu Erwartungen, die Leistungen der Schülerinnen und Schüler beeinflussen zu können (Lehrerwirksamkeit), zum Wesen des Wissens (epistemologische Überzeugungen) oder $\mathrm{zu}$ den Ursachen von Lehrer- und Schülerleistung (Attributionen). In neueren Arbeiten (z.B. Speer, 2005) werden ähnlich wie schon bei Keber (1997) und bei Sfard (1998) folgende Aspekte genannt: die Lehrkraft und das Lehren sowie die Lernenden und das Lernen. Ausgehend von diesen Arbeiten konzentriert sich die vorliegende Arbeit ebenfalls auf beide Aspekte: auf das Lehren - also auf die Lehrkraft und ihre Rolle bzw. ihre Aufgaben - sowie auf das Lernen und die Rolle und Aufgaben der Lernenden.

\subsubsection{Lehrerprofessionsdebatte und Lehrerüberzeugungen im Handlungskompetenzmodell der Lehrkräfte}

Lehrerüberzeugungen haben nicht nur Eingang in die empirische Forschung, sondern auch in die theoretische Debatte um Lehrerprofessionalität gefunden. Gerade in den letzten Jahren nahm die Diskussion um den Lehrerberuf durch den Bericht des Panel Research and Teacher Education „Studying Teacher Education“ (vgl. Cochran-Smith \& Zeichner, 2005) der American Educational Research Association (AERA) und des Panel „Preparing Teachers for a Changing World" (vgl. Darling-Hammond \& Bransford, 2005) der National Academy of Education internationales Ausmaß an. Aus dem deutschsprachigen Raum sind zwei zentrale Arbeiten zu nennen: der Abschlussbericht der Lehrerbildungskommission der Kultusministerkonferenz „Perspektiven der Lehrerbildung in Deutschland“ (vgl. Terhart, 2000) und der schweizerische Forschungsbericht „Die Wirksamkeit der Lehrerbildungssysteme“ (vgl. Oser \& Oelkers, 2001). 
Hierzulande verläuft die Debatte um Lehrerprofession unter der Kontroverse des strukturtheoretischen Ansatzes des Lehrerhandelns (Oevermann, 1996) und des generischen Modells professioneller Handlungskompetenzen der Lehrkräfte (Baumert \& Kunter, 2006). Ersterer beschreibt das Unterrichtsgeschehen als sozialisatorische Interaktion zwischen der Lehrkraft und ihren Schülerinnen und Schülern (vgl. Helsper, 2004; Oevermann, 1996; Radtke, 2004; Kolbe, 2004). Diese durch emotionale Zuwendung gekennzeichnete Beziehung betrachtet den Schüler in seiner ganzen Persönlichkeit. Im Gegensatz dazu werden im generischen Modell die unterrichtsbezogenen Aufgaben betont. Der Schüler wird nicht vor seinem biografischen Hintergrund, also ganzheitlich, wahrgenommen, sondern als Teilnehmer eines Bildungsprogramms (Fend, 2006; Leschinsky \& Cortina, 2008). Das Unterrichten selbst stellt hier die zentrale Aufgabe der Lehrkraft dar, wodurch das systematische Lernen erreicht werden soll, welches die kognitiven und motivationalen Voraussetzungen beruflicher, politischer und kultureller Teilhabe am Gesellschaftsleben für die nachwachsende Generation sichern soll (Baumert \& Kunter, 2006). Im Handlungskompetenzmodell der COACTIVStudie wird Lehrerkompetenz als das ,dynamische Zusammenwirken von Aspekten des Professionswissens, Überzeugungen, motivationalen Orientierungen und selbstregulativen Fähigkeiten“ definiert (Brunner et al., 2006, S. 523) (siehe Abb. 1).

\section{Abbildung 1:}

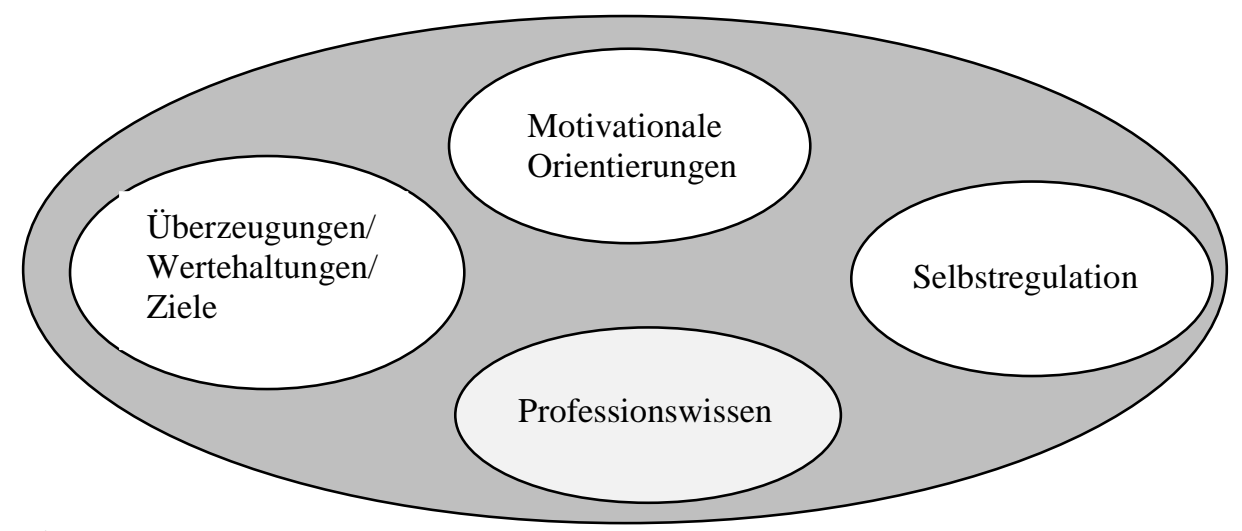

Das professionelle Handlungskompetenzmodell von Lehrkräften von COACTIV (Kunter et al., 2011, S. 32).

Lehrerüberzeugungen werden hier explizit als Teil von Lehrerkompetenz behandelt, wobei, ähnlich wie in den früheren Definitionen (vgl. Nisbett \& Ross, 1980; Nespor, 1987), eine kategoriale Trennung der Facetten Wissen und Werthaltungen/Überzeugungen vorgenommen wird (Kunter et al., 2011). Zu den Überzeugungen zählen: 1) epistemologische Überzeugungen, die sich auf die Struktur, Genese und Validierung von Wissen beziehen, 2) 
Überzeugungen über das Lernen (subjektive Lerntheorien), 3) subjektive Theorien über das Lehren und 4) selbstbezogene Überzeugungen hinsichtlich des Lernens und Lehrens selbstbezogene Fähigkeitskognitionen (Kunter et al., 2011, S. 41). Hinzu kommen Zielvorstellungen von Curriculum und Unterricht (Aebli, 1983; Klafki, 1996; Klauer \& Leutner, 2007; Reusser, 2008) sowie Wertbindungen, die die besondere Berufsethik mit Fürsorge, Gerechtigkeit und Wahrhaftigkeit im Sinne einer Berufsmoral wiedergeben (Oser, 1998, 2009). Dabei stehen zum Beispiel Fürsorge sowohl für Nachsicht (Fördern) als auch für Voraussicht (Fordern) und Gerechtigkeit für Verteilungsgerechtigkeit, Gleichheit, Billigung und Reziprozität (Oser, 1998, 2009; Rechenbach, 1994). Baumert und Kunter (2006) weisen explizit darauf hin, dass bis heute ein klarer forschungsfundierter Wissensstand darüber fehlt, welche Bedeutung spezifische Wertbindungen für Lehrkräfte haben.

\subsubsection{Lehrerüberzeugungen zum Lehren und Lernen. Eine Begriffsklärung}

Die Lehrerprofessionsdebatte ist im Kontext der vorliegenden Arbeit von besonderer Wichtigkeit, weil sie Lehrerüberzeugungen zum Lehren und Lernen explizit als Teil von Lehrerprofessionskompetenz definiert und so die Bedeutung der Lehrerüberzeugungen für den Lehrerberuf unterstreicht. Insgesamt sind in Bezug auf die vorliegende Arbeit die Studien aus dem COACTIV-Projekt hervorzuheben (Baumert \& Kunter, 2006), die dem Thema Lehrerüberzeugungen mehr Aufmerksamkeit gewidmet haben. In erster Linie sind im COACTIV-Projekt und auch bei Shulman (1986, 1987), auf dessen Konzeptualisierung des Professionswissens das COACTIV-Modell basiert, die fachlichen und fachdidaktischen Facetten des Fachwissens von zentraler Bedeutung. Durch die Definition von Lehrerüberzeugungen als ein Teil professioneller Kompetenz von Lehrkräften gewinnen sie an Bedeutung im Kontext empirischer Forschung, so dass diese in einigen groß angelegten empirischen Studien untersucht wurden (z.B. Dubberke et al., 2008; Hartinger et al., 2006; Seidel et al., 2008).

So wird in der definitorischen Frage an die Arbeiten aus dem COACTIV-Projekt angeknüpft. Im Rahmen der vorliegenden Arbeit werden Lehrerüberzeugungen als implizite und explizite, subjektiv für wahr gehaltene Konzeptionen verstanden, welche die Wahrnehmung der Umwelt und das Handeln beeinflussen (Kunter et al., 2011). Zusätzlich wird diese Definition durch die Benennung der dazu gehörigen Aspekte präzisiert: Lehrerüberzeugungen zum Lehren und Lernen sind subjektive, implizite und explizite 
Vorstellungen über das Lehren, also über die Lehrkraft und ihre Rolle bzw. ihre Aufgaben sowie über das Lernen mit den Rollen und den Aufgaben der Lernenden.

\subsubsection{Verschiedene Lehr-Lern-Überzeugungen}

Im Vorfeld der empirischen Arbeiten in der vorliegenden Dissertation wurde eine umfassende Literaturrecherche zu Lehrerüberzeugungen durchgeführt. Auffallend waren dabei zum einen die vielen Parallelbezeichnungen und zum anderen eine beträchtliche Menge an verschiedenen Überzeugungen zum Lehren und Lernen. Oft werden verschiedene Begriffe für die Bezeichnung ähnlicher Inhalte verwendet: „Comparison of the labels and descriptions [...] indicates a high degree of commonality in [them]. Precisely the same wording or terminology may not be used, but it seems reasonable to interpret [beliefs] as common if the descriptors appear to have the same or similar meanings." (Kember, 1997, S. 259) So wurden zum Beispiel in der Arbeit von Alger (2009) und Kember (1997) die in der Literatur identifizierten Konzeptionen verschiedener Studien miteinander verglichen und aufgrund ihrer inhaltlichen Ähnlichkeit zu einigen wenigen zusammengefasst. In Anlehnung an diese Vorgehensweise, wurden in der vorliegenden Arbeit die bei der Literaturrecherche identifizierten Überzeugungen gegenübergestellt und deren Inhalte miteinander verglichen. Es wurde eine Reduktion der Anzahl verschiedener Überzeugungen angestrebt, ohne die Inhalte der einzelnen Überzeugungen zu reduzieren. An dieser Stelle sei betont, dass die entdeckten Relationen keinen Anspruch auf Ausschließlichkeit der Vergleiche erheben. Einen ausführlichen Überblick über die vorgefundenen Überzeugungen in ihrer Relation zueinander bietet eine Tabelle im Anhang A.

Die meisten Studien, die sich mit Lehrerüberzeugungen zum Lehren und Lernen befassen, unterscheiden zwei Überzeugungen: knowledge transmission und cognitive construction (Chan \& Elliott, 2004; Dubberke et al., 2008; Norton et al., 2005; OECD, 2009; Sfard, 1998; Kunter et al., 2011; Peterson et al., 1989; Staub \& Stern, 2002).

Bei der Überzeugung knowledge transmission richtet sich der Fokus auf Lerninhalte und Kenntnisse, die zum Eigentum eines Schülers werden. Als Metapher dieser Überzeugung wird das Bild des „Nürnberger Trichters“ verwendet, der menschliche Geist wird dabei mit einem „Container“ verglichen (Sfard, 1998). Synonym zur Transmission sind zu verstehen: knowledge transmission (Norton et al., 2005; Pratt, 1992, 2005), transmitting structured knowledge (Kember, 1997) oder auch acquisition (Sfard, 1998) und acquisition and reproduction of content (Boulton-Lewis et al., 2001), wobei die letzteren Begriffe die 
Lernerseite ansprechen. Die Überzeugung Transmission geht auf die Lehr-Lern-Theorie des Behaviorismus oder die assoziationistische Theorie zurück (Staub \& Stern, 2002), wonach Reiz-Reaktions-Modelle das Lernen erklären und Verstärkerpläne als zentrales Element des Lernens aufgefasst wurden (Skinner, 1973, 1978). Das Lehren wird im Sinne eines SenderEmpfänger-Modells als Übertragung von (strukturierten) Lerninhalten von der Lehrkraft zu den Schülerinnen und Schülern verstanden: Die Lehrkraft präsentiert Lerninhalte, und die Schülerinnen und Schüler nehmen diese auf. Dabei ist die Rolle der Schülerinnen und Schüler eine passiv-rezeptive (imparting information bei Kember, 1997; structuring learning bei Dunkin \& Precians, 1992).

Die andere, gut erforschte Überzeugung zum Lehren und Lernen geht auf die konstruktivistische Lehr-Lern-Theorie zurück (Staub \& Stern, 2002) und wird als constructivist view (Staub \& Stern, 2002) oder als cognitive view (Martínez et al., 2001) bezeichnet. Hier wird Lernen als ein aktiver und selbstverantwortlicher Prozess der EigenKonstruktion des Wissens durch den Schüler verstanden, und das Lehren wird aus der Sicht des Schülers und seiner Entwicklung gesteuert. Konzeptentwicklung stellt dabei einen zentralen Aspekt des Lernens im Konstruktivismus dar (Ginsburg \& Otto, 1991; Piaget, 1978), entsprechend finden sich Bezeichnungen wie developmental (Pratt, Collins \& Jarvis Selinger, 2001) oder conceptual change (Kember, 1997). Verglichen mit der TransmissionsAuffassung verschiebt sich der Fokus vom Lehrer zum Schüler (Trigwell et al., 1994), von Wissensübernahme zum Verstehen der Lerninhalte wie in facilitation understanding (Kember, 1997) oder learning facilitation (Norton et al., 2005).

Ein großer Teil der Studien, die sich mit Transmission und Konstruktion befassen, ist quantitativ, was den Vorteil hat, die Überzeugungen reliabel und valide an großen Stichproben messen, sowie Zusammenhänge der beiden Überzeugungen mit für den Kontext des schulischen Lernens relevanten Variablen analysieren zu können, wie zum Beispiel zum Lehrerhandeln und zur Schülerleistung (vgl. Dubberke et al., 2008; Hartinger et al., 2006; Seidel et al., 2006; Staub \& Stern, 2002; Peterson et al., 1989).

Verglichen mit den Überzeugungen Transmission und Konstruktion fanden andere Überzeugungen zum Lehren und Lernen bisher nur vereinzelt Beachtung und wurden meist nur qualitativ untersucht (Pratt et al., 2001; Martínez et al., 2001). Schülerorientierung (Klieme et al., 2006) und Partizipation (Lave \& Wenger, 1991) stellen solche wenig untersuchten Überzeugungen dar. Gleichwohl wurden deren zentrale Gedanken, wenn auch unter anderen Bezeichnungen, in einigen theoretischen und empirischen Arbeiten $\mathrm{zu}$ Lehrerüberzeugungen beschrieben (participation bei Sfard, 1998; establishing interpersonal 
relationship bei Dunkin \& Precians, 1992). Sfard (1997) legt dar, dass in der Wissenschaft eine allgemeine Veränderung des Verständnisses vom Lernen stattfand: Während in der transmissiven Sicht auf das Lernen eines Individuums das Besitzen dieses Wissens im Zentrum stand, wurden Prozesse der Teilhabe an Aktivitäten einer Lerngemeinschaft in der partizipatorischen Sicht auf das Lernen entscheidender. Theoretisch lässt sich das Lernen als Partizipation in den Lerngemeinschaftsansatz (engl. Legitimate Peripheral Participation/Community of Practices) nach Lave und Wenger (1991) sowie in die interaktive Lernmethode Apprenticeship einordnen (Collins, Brown \& Newman, 1989; Palincsar \& Brown, 1984; Rogoff, 1990; Scardamalia \& Bereiter, 1987). Diese Lernansätze der Theorie des situierten Lernens (Brown, Collins \& Duguid, 1989) legen den Fokus auf die soziale Dimension des Lernens. So wird das Lernen in der partizipatorischen Vorstellung als Mitglied-Werden in einer Gemeinschaft definiert, als Enkulturation eines neuen Mitglieds durch Übernahme von Verhaltensregeln und durch die Verwendung einer mit dieser Gemeinschaft gemeinsamen Sprache (Lave \& Wenger, 1991). Es wird vom Interesse des Lerners an den Aktivitäten der Gemeinschaft ausgegangen, anstatt vom Bestreben nach einer persönlichen Bereicherung durch Wissensaneignung (Lave \& Wenger, 1991; Sfard, 1998). So ist auch nicht das Endresultat des Lernens von Bedeutung, sondern die ständige authentische Teilhabe an den Aktivitäten einer Arbeits- und Lerngemeinschaft, in der das Wissen durch den immer währenden Dialog, den Austausch und die Zusammenarbeit unter den Mitgliedern gemeinsam ausgehandelt wird (Martínez et al., 2001).

Die meisten Studien, die die partizipatorische Überzeugung darstellen, sind qualitativ (Alger, 2009; Martínez et al., 2001). Ihre Ergebnisse können nur eingeschränkt miteinander verglichen werden. Zudem ist es schwierig, den Einfluss dieser Überzeugung auf relevante Variablen aus dem schulischen Kontext, wie zum Beispiel das Lehrerhandeln im Unterricht oder die Schülerleistung, zu untersuchen. Die Arbeit von Pratt et al. (2001) stellt hier eine Ausnahme dar. Die Autoren entwickelten einen Fragebogen, den „Teaching Perspective Inventory“ (TPI). Mithilfe dieses Fragebogens wurden verschiedene - unter anderem auch partizipatorische und schülerorientierte - Perspektiven untersucht. Allerdings fehlen für das TPI entsprechende Validierungsstudien und auch der Versuch der Übersetzung ins Deutsche blieb erfolglos (Hanke \& Schwarz, 2011). Pratt et al. (2001) definieren Schülerorientierung (engl. nurturing) als eine psychologisch, emotional und auch physikalisch sichere, das Selbstwertgefühl des Schülers unterstützende Lernumgebung. Dabei sind ein warmes, unterstützendes Lehrerverhalten und eine individuelle Unterstützung des Schülers wichtig (Klieme et al., 2006), ähnlich dem in den 1960er Jahren entstandenen pädagogischen Ansatz 
des Humanismus (Huitt, 2009; Rogers, 1969; Rogers \& Freiberg, 1994). In diesem wird der Lernende in seiner ganzen Person aufgefasst und seine persönliche Entwicklung zum Ziel des Lernens erklärt. Emotionen treten dabei als Regulationsmechanismen zwischen der Lernumgebung und den Lernprozessen auf (Huitt, 2009): Fehlerangst zum Beispiel beeinflusst das Lernverhalten negativ, dagegen ist ein produktiver Umgang mit Schülerfehlern empfehlenswert (Assor, Kaplan \& Roth, 2002; Deci \& Ryan, 1987; Gruehn, 2000; Klieme et al, 2006).

Nach Klieme et al. (2006) ist ein Lernklima unterstützend und schülerorientiert, wenn die Bedürfnisse der Schülerinnen und Schüler hinsichtlich der Autonomie, dem Erleben eigener Kompetenz und der sozialen Eingebundenheit beachtet werden (siehe auch Selbstbestimmungstheorie der menschlichen Motivation von Deci \& Ryan, 1993). Dem Autonomiebedürfnis kann im Unterricht durch eine Mitsprachemöglichkeit der Schülerinnen und Schüler bei der Auswahl von Unterrichtsthemen und Aufgaben begegnet werden (Huitt, 2009; Klieme et al., 2006; vgl. auch Dunkin \& Precians, 1992: encouraging activity and selfdirected learning). Durch das eigenständige Lernen gewinnen die Schülerinnen und Schüler die Einsicht, dass ihr eigener Einsatz für den Lernerfolg entscheidend ist (Pratt et al., 2001), was den Glauben an die eigene Lernfähigkeit stärkt (Deci \& Ryan, 1985).

Das Bedürfnis nach dem Erleben der eigenen Kompetenz entspricht dem Gefühl, den sich stellenden Anforderungen gewachsen zu sein (Deci \& Ryan, 1987). Wenn der Schüler den Unterschied zwischen eigenem Wissensstand und dem Schwierigkeitsgrad der Aufgabe als zu bewältigen einschätzt, ist eine optimale Herausforderung ohne Angstgefühle und Druckempfinden gegeben (Pratt et al., 2001; Pratt, 2005; Waldis, Grob, Pauli \& Reusser, 2010). Somit ist wichtig, dass die Lehrkraft das Leistungsniveau ihrer Schülerinnen und Schüler gut kennt, um sie entsprechend zu fördern und zu fordern (Pratt, 2002).

Das Bedürfnis nach befriedigenden Sozialkontakten und sozialer Eingebundenheit bezieht sich auf die subjektiv wahrgenommene Beziehung zu wichtigen Personen und Gruppen. Wird diese als zufriedenstellend empfunden, engagieren sich Schülerinnen und Schüler für schulische Ziele und Anforderungen (Deci \& Ryan, 1987). Die Lehrkraft hat daher sowohl für einen respektvollen und wertschätzenden Umgang mit den Schülerinnen und Schülern zu sorgen als auch dafür, dass der Umgang der Schülerinnen und Schüler untereinander respektvoll verläuft (Ryan \& Patrick, 2001). In der Studie von Waldis et al. (2010) wird ein demokratischer Interaktionsstil der Lehrkraft als wertschätzend, respektvoll und einfühlsam beschrieben (engl. caring). Eine gute Lehrkraft gibt Rückmeldung unter Berücksichtigung der persönlichen Situation des Schülers, ist ehrlich, gerecht, 
vertrauenswürdig und fürsorglich ihren Schülerinnen und Schülern gegenüber (Assor et al., 2002; Ryan \& Deci, 2000; Wentzel, 1997).

Zusammenfassend können vier Gruppen der lehr-/lernbezogenen Überzeugungen genannt werden, welche auf unterschiedliche theoretische Paradigmen zurückführen: 1) die Überzeugung knowledge transmission auf die behavioristische Lehr-Lern-Theorie, 2) die cognitive construction oder developmental Überzeugung auf den Konstruktivismus, 3) participation auf den Lerngemeinschaftsansatz (Lave \& Wenger, 1991) und den Apprenticeship-Lernansatz (Collins et al., 1989; Palincsar \& Brown, 1984; Scardamalia \& Bereiter, 1987) sowie 4) nurturing auf das Konstrukt der Schülerorientierung nach Klieme et al. (2006) oder auch auf die humanistische Lerntheorie (Huitt, 2009). Die in der empirischen qualitativen und quantitativen Forschung unter den unterschiedlichsten Bezeichnungen dargestellten Perspektiven, Konzeptionen und Metaphern lassen sich auf diese vier Gruppen zurückführen. Im Weiteren werden dafür deutschsprachige Begriffe verwendet: Transmission, Konstruktion, Partizipation und Schülerorientierung. Für die letzte Überzeugung ist es schwierig, eine eindeutige deutsche Übersetzung $\mathrm{zu}$ finden. Es kämen folgende Bezeichnungen in Frage: Pflege, Erziehung oder Bildung. Diese Begriffe bergen jedoch das Risiko, irreführende Konnotationen hervorzurufen. Daher wird diese Überzeugung in Anlehnung an Klieme et al. (2006) mit dem Begriff Schülerorientierung übersetzt. 


\section{2 Überblick über den aktuellen Forschungsstand zu Lehr-Lern-Überzeugungen}

Den Lehr-Lern-Überzeugungen von Lehrkräften wurde vor allem in der amerikanischen Forschung viel Aufmerksamkeit geschenkt, mit dem Ergebnis einer Reihe theoretischer (z.B. Borko \& Putnam, 1996; Pajares, 1992) und empirischer Arbeiten (z.B. Alger, 2009; Aguirre \& Speer, 2000; Leavy et al, 2007; Speer, 2005; Stipek et al., 2001). Aus Nordamerika sind die Arbeiten von Pratt et al. (2001) und Wideen et al. (1998) und aus Australien die Arbeiten von Trigwell und Prosser (2004), aber auch eine neuere Studie von Wan Ng et al. (2010) nennenswert. Zudem existieren zahlreiche Studien aus dem europäischen Raum (aus England: Kember, 1997; Norton et al, 2005; der Schweiz: Staub \& Stern, 2002; den Niederlanden: Meirink et al., 2009; van Driel, Bulte, Verloop, 2007; Finnland: Postareff \& Lindblom-Ylänne, 2008; Schweden: Dahlgren \& Chiriac, 2009; Spanien: Martínez et al, 2001; Griechenland: Potari, 2009). Im deutschsprachigen Raum wurde das Interesse an Lehrerüberzeugungen deutlich stärker, seit das Konzept Eingang in die Debatte um das professionelle Lehrerwissen (Bromme, 1997) und in die bundesweite und internationale Schulvergleichsstudie TIMSS (vgl. Köller et al., 2000) gefunden hat. Lehrerüberzeugungen wurden im DFG-Schwerpunktprogramm „Bildungsqualität von Schule“ (BiQua) (z.B. Prenzel, 2007) und dem COACTIV-Projekt (z.B. Baumert \& Kunter, 2006) als ein Teil professioneller Kompetenz von Lehrkräften definiert und sind somit in ihrer Bedeutung im Zusammenspiel mit fachwissenschaftlichem und fachdidaktischem Wissen von Lehrkräften in Hinblick auf curricular valide gemessene Schulleistungen in den Fokus wissenschaftlicher Forschung gerückt.

Die bisherige Forschung zu Lehr-Lern-Überzeugungen befasst sich mit verschiedenen Aspekten. Allgemeine Studien, welche der Frage nach den Überzeugungen von Lehrkräften nachgehen, analysieren Inhalte von Überzeugungen interviewter Lehrkräfte (Alger, 2003; Leavy et al., 2007). Die meisten Studien mit der Frage nach der Entwicklung der Lehr-LernÜberzeugungen konnten nur anhand der Vergleiche von verschiedenen Lehrkräften, zum Beispiel mit unterschiedlich umfangreicher Lehrerfahrung durchgeführt werden (Leavy et al., 2007; Martínez et al., 2001; Saban, 2010; Saban et al., 2007). Studien mit der Frage nach der Veränderbarkeit der Lehr-Lern-Überzeugungen versuchen beispielsweise im Anschluss an ein Studienjahr oder an eine Intervention eine Veränderung der Überzeugungen zu dokumentieren (Hollingsworth, 1989; Nettle, 1998; Rust, 1994; Weinstein, 1990; Wideen et al., 1998). Besonders im Kontext des schulischen Lernens scheint der Einfluss der Lehrerüberzeugungen auf unterrichtliches Handeln der Lehrkräfte (z.B. Clark, 1988; Cole, 
1989; Fenstermacher, 1979; Nespor, 1987; Pintrich, 1990) und auf das Schülerlernen (Hartinger et al., 2006) sowie die Schülerleistung von Bedeutung zu sein. Die beiden Forschungsrichtungen - zur Entwicklung von Lehrerüberzeugungen und zu ihren Einfluss auf die Schülerleistungen - werden im Folgenden gesondert dargestellt.

\subsubsection{Entwicklung von Lehr-Lern-Überzeugungen während der Lehrerlaufbahn}

Seit den ersten Arbeiten zum Thema Lehrerüberzeugungen wird über deren Entwicklung bzw. über die Möglichkeit, die Überzeugungen zu verändern, kontrovers diskutiert (z.B. Pajares, 1992; Nisbett \& Ross, 1980). Allgemein wird davon ausgegangen, dass sich Überzeugungen im Verlauf der Sozialisation ausbilden (z.B. Calderhead \& Robson, 1991; Feiman-Nemser \& Remillard, 1996). Pajares (1992) stellte fest, dass sich Überzeugungen zum Lehren und Lernen nicht einfach und schnell verändern lassen, dass sie sich ,at the core of teachers' world view“ befinden (S. 311). Überzeugungen werden als hochgradig resistent gegen Veränderungen betrachtet (Nisbett \& Ross, 1980), und wenn sie nicht bewusst in Frage gestellt werden, bleiben sie unverändert (Lasley, 1980). Tendenziell sind Personen demnach bestrebt, ihre bestehenden Überzeugungen beizubehalten und auch neue Informationen, die als Beweis gegen die bestehenden Überzeugungen interpretiert werden müssten, als Bestätigung der alten Überzeugungen zu verwenden (Nisbett \& Ross, 1980). So bleiben sie oft unverändert, obwohl eine Veränderung zu erwarten wäre (Pajares, 1992). Diese Aussagen wurden allerdings auf der philosophisch-theoretischen Ebene getroffen. Die empirische Forschung konnte bisher zu keinem Konsens in dieser Frage gelangen.

Hinweise auf die Entwicklung von Überzeugungen können aus Untersuchungen derselbigen zu unterschiedlichen Zeitpunkten in der Lehrerlaufbahn abgeleitet werden. Im Folgenden werden Ergebnisse verschiedener Studien vorgestellt, angefangen mit Untersuchungen bei zukünftigen Lehrkräften am Anfang ihrer Lehrerausbildung über das Studium hinweg bis hin zu Vergleichsstudien von Studierenden und Lehrkräften mit Erfahrung.

Studien mit angehenden Lehrkräften (Lehrkräfte in ihrer Lehrerausbildung) sind meist qualitativ oder basieren auf Fallbeispielen. In diesen Studien wurden die Überzeugungen angehender Lehrkräfte als transmissiv, aber auch liberal und humanistisch beschrieben: Lehren wurde mit telling und mit mechanical transfer of information gleichgesetzt (Brookhart \& Freeman, 1992; Calderhead, 1996; Richardson, 1996; Russel, 1988) bzw. Lernen als 
memorization of material verstanden (Black \& Ammon, 1992). Angehende Lehramtsstudierende schätzten Aspekte der Schüler-Lehrer-Beziehung als wichtiger ein als akademische Aspekte (Brookhart \& Freeman, 1992). Ferner zählten sie Warmherzigkeit, Mitgefühl, Freundlichkeit und die Fähigkeit, sich in die Lage des Schülers zu versetzen, zu den Eigenschaften einer guten Lehrkraft (Rathbone \& Pierce, 1990; Weinstein, 1989). In ihrem Review-Artikel stellten Wideen et al. (1998) fest, dass angehende Lehrkräfte besonders die Schüler-Lehrer-Beziehung sowie die Beziehungen unter den Schülerinnen und Schülern, aber auch das Selbstkonzept des Schülers und helfendes Lehrerverhalten für wichtig erachteten. Auch in einer aktuellen Studie von Wan $\mathrm{Ng}$ et al. (2010) mit Lehramtsstudierenden wurden eine charismatische Ausstrahlung der Lehrkraft sowie ihre Fähigkeit, eine Beziehung zu Schülerinnen und Schülern aufzubauen, als wichtige Charakteristiken genannt. Die vorgestellten Studien legen demnach nahe, dass sich in den Überzeugungen angehender Lehrkräfte die Perspektiven Transmission und Schülerorientierung zeigen.

Quantitative Studien, die sich mit der Entwicklung von Überzeugungen angehender Lehrkräfte während der universitären Ausbildung beschäftigten, lieferten sowohl Hinweise auf Veränderung als auch auf Stabilität von Überzeugungen (Nettle, 1998, S. 193). In seiner Fallstudie begleitete Hollingsworth (1989) 14 amerikanische Studierende während des ersten Jahres ihrer Lehrerausbildung. Die Ergebnisse illustrierten eine Veränderung der Überzeugungen in Richtung constructivist and participatory learning. Weinstein (1990) berichtete von ausbleibenden Veränderungen der Überzeugungen von Studierenden während eines siebenwöchigen Kurses. Im Gegensatz dazu sprach Nettle (1998) von einer Veränderung der Überzeugungen: Bereits nach drei Wochen Schulpraktikum während ihrer Lehrerausbildung für Grundschulen änderten 79 australische Studierende ihre affectiveoriented beliefs zugunsten von task-oriented beliefs. Ebenso wurde von einer Veränderung in den Ansichten hinsichtlich des Lehrerberufs vom „Helfen“ zum „Organisieren von Klassenprozessen“ in der Studie von Rust (1994) berichtet; auch hier verloren die Überzeugungen von Studierenden ihre nurturing aspects.

Einige Studien analysierten Lehr-Lern-Überzeugungen, indem sie metaphorische Vorstellungen vom Lehren und Lernen untersuchten (z.B. Leavy et al., 2007; Saban, Kocbeker \& Saban, 2007). In der Studie von Saban et al. (2007) wechselten 1142 türkische Studierende nach einem Studienjahr ihre nurturing orientierten Lehrermetaphern (z.B. Gärtner) gegen Lehrermetaphern konstruktivistischer Natur (z.B. Leuchtturm). Im Gegensatz dazu zeigte die Folgestudie von Saban (2010) zu Schüler-Metaphern, dass die Studierenden 
am Ende des Studiums eine transmissive Vorstellung aufwiesen (Saban, 2010). Leavy et al. (2007) untersuchten metaphorische Vorstellungen bei 124 irischen und amerikanischen Studierenden. Am Anfang des Studienjahres verdeutlichte die Mehrheit der Metaphern (49\%) eine behavioristisch-empiristische Perspektive auf das Lehren (z.B. Kochen nach Rezept), die von einer kognitiv-konstruktivistischen (z.B. Entwicklung eines Tänzers) (24\%) sowie von einer situativ-soziohistorischen Perspektive (z.B. Rucksackreise) (9\%) gefolgt wurde. Die in dieser Studie untersuchte soziohistorische Perspektive basiert auf partizipatorischen Ideen des Lernens als ein Produkt gemeinschaftlichen Wissensaufbaus in der Lerngruppe (siehe auch Situierte Kognition: z.B. Greeno, Collins \& Resnick, 1996). Die am Ende des Studienjahres erhobenen Metaphern entsprachen zu 44 Prozent der konstruktivistischen Sicht, zu 42 Prozent der behavioristischen und zu sechs Prozent der soziohistorischen Sicht auf das Lehren und Lernen. Somit lässt sich eine Veränderung innerhalb eines Studienjahres weg von transmissiven hin zu konstruktivistischen Vorstellungen festhalten. Australische Studierende in der Studie von Wan Ng et al. (2010) hielten am Anfang ihres Studiums fachliche und didaktische Kompetenzen des Lehrers für wichtig, so zum Beispiel ,to know what and how to teach“ (S. 286), „prepare lesson plans“ (S. 287), „,build rapport, manage students“ (S. 283). Im Verlauf des Studiums mit Hospitationsphasen als eigenem Unterricht in der Schule veränderte sich diese Meinung und die Studierenden schätzten die Fähigkeit, die Klasse durch eine charismatische Ausstrahlung kontrollieren zu können, als wichtiger ein. Die Beispiele „able to relate to students“ (S. 286), „to helm students succeed“ (S. 288), „listen more than they tell“ (S. 288) sprechen Inhalte der schülerorientierten Überzeugung an. Dieses Ergebnis zeigt, dass die Studierenden ihre Präferenz für transmissive Vorstellungen zugunsten einer Schülerorientierung aufgaben.

Die genannten Studien fokussieren die Überzeugungen und deren Entwicklung während der Lehrerausbildung. Insgesamt lässt sich festhalten, dass die Studien mit dem Schwerpunkt auf den Verlauf des Studiums für eine Veränderung von lehr-/lerntheoretischen Überzeugungen während des Studiums sprechen, allerdings ist die Befundlage inkonsistent. Im Folgenden werden Untersuchungen dargestellt, die sich mit den Überzeugungen von Lehrkräften im Beruf befassen.

Zunächst ist anzumerken, dass behavioristische Lernmodelle und Überzeugungen in den Bildungswissenschaften oftmals kritisiert werden, während der Konstruktivismus als gegenwärtige Lehr-Lern-Theorie dominiert. Somit wurden allgemein Entwicklungen der Überzeugungen in Richtung Transmission als weniger wünschenswert betrachtet und eine ungenügende Entwicklung konstruktivistischer Überzeugungen während der 
Lehrerausbildung kritisiert (vgl. Reviews von Nettle, 1996; Wideen et al., 1998). Anstatt sich neue Überzeugungen zum Lehren und Lernen anzueignen, lernten Studierende ihre existierenden Überzeugungen gegen neue Informationen zu verteidigen (Stofflett \& Stoddart, 1991). Ebenfalls massiv kritisiert wurde im US-amerikanischen Raum die Lehrerausbildung (Richardson, 1996, 2003). Sobald angehende Lehrkräfte nach ihrem Studium in den Beruf einsteigen, verschwindet ein Großteil des während der Lehrerausbildung Erlernten im Umfeld der neuen Schule (Kagan, 1992; Zeichner \& Tabachnik, 1981). Eine mögliche Erklärung liefert der „Praxisschock“, auf welchen junge Lehrkräfte in ihren ersten Erfahrungen mit der Berufspraxis treffen. Von diesem Phänomen wurde bereits 1978 in der deutschen Studie von Dann, Cloetta, Müller-Fohrbrodt und Helmreich berichtet. Praxisschock beschreibt, wie Studierende mit verschiedenen unbekannten Situationen im Klassenzimmer und der neuen Schule konfrontiert und von negativen Emotionen wie Unsicherheit begleitet werden (Potari \& Georgiadou-Kabouridis, 2009). Sie erleben oft Diskrepanzen zwischen der Universität und der Schule (Cooney, 1985; Raymond, 1997). Das an der Universität Gelernte und die schulischen Prioritäten passen nur selten zusammen (Skott, 2002). Dies zwingt die jungen Lehrkräfte dazu, ihre innovativen Überzeugungen und Unterrichtsmethoden zu ändern und sich der Tradition der Schule anzupassen (Potari \& Georgiadou-Kabouridis, 2009). So werden die progressiveren pädagogischen Einstellungen, die durch die Lehrerausbildung geprägt wurden, direkt nach den ersten Kontakten mit der Berufspraxis aufgegeben (z.B. Dann et al., 1978; Koch, 1972). Durch den Praxisschock gewinnen konservative Einstellungen auf lange Sicht die Oberhand und manifestieren sich so dauerhaft in der Praxis der Lehrkräfte. Unter „konservativen Lehrereinstellungen“ wurden in der Studie Druckorientierung und Disziplinierungsmaßnahmen, Reformskepsis sowie Anlageorientierung verstanden (Dann et al., 1978). Insgesamt zeigten die Ergebnisse der Längsschnittstudie von Dann et al. (1978, 1981), dass ein Prozess des allmählichen Konservativer-Werdens direkt nach dem Einstieg in den Beruf einsetzte und auch vier Jahre später nicht stagnierte: Junge Lehrkräfte waren vier Jahre nach dem Berufseinstieg ,sowohl in ihrer allgemeinen weltanschaulichen Grundhaltung als auch in ihren spezifischen Werthaltungen und erziehungs- und schulbezogenen Einstellungen im Durchschnitt noch konservativer als sie es nach einem Jahr Berufspraxis schon waren“ (Dann et al., 1981, S. 258).

Bisher konnte die empirische Forschung auf Unterschiede zwischen Lehr-LernÜberzeugungen zukünftiger Lehrkräfte in ihrer universitären Ausbildung und denen der schon im Beruf stehenden Lehrkräfte hinweisen. Während fortschrittliche Sichtweisen auf das Lehren und Lernen - wie konstruktivistische und partizipatorische Überzeugungen - von 
zukünftigen Lehrkräften gezeigt wurden, verfügten Lehrkräfte mit mehreren Jahren Berufserfahrung eher über das traditionelle Bild vom Lehren und Lernen. Martínez et al. (2001) verglichen in ihrer Studie konzeptuelle Metaphern des Lehrens und Lernens von 50 erfahrenen Lehrern mit 38 Studierenden in ihrer Lehrerausbildung in Spanien. Auf der einen Seite illustrierte die Mehrheit der von erfahrenen Lehrkräften produzierten Metaphern (57\%) behavioristische Einsichten, während es von solchen Metaphern bei Studierenden deutlich weniger gab (22\%). Auf der anderen Seite äußerten zukünftige Lehrkräfte mehr konstruktivistische Ideen (56\%) als erfahrene Lehrkräfte (38\%). Ebenfalls wurden von zukünftigen Lehrern mehr partizipatorische Vorstellungen (20\%) als von erfahrenen Lehrern (ca. 2\%) angesprochen. In der Längsschnittstudie von Meirink et al. (2009) mit 34 niederländischen Lehrkräften aus dem Sekundarschulbereich wurde bei 13 von 34 Lehrkräften keine Veränderung der Überzeugungen nach einem Jahr festgestellt, ebenso nicht nach einem halben Jahr in der Studie von Boulton-Lewis et al. (2001) mit australischen Lehrkräften aus dem Bereich der Sekundarschule. So wie in den Studien aus Spanien (Martínez et al., 2001) und der Türkei (Saban, 2010; Saban et al., 2007) wurden in der Studie von Alger (2009) mit 110 amerikanischen Lehrkräften konzeptuelle Metaphern als methodischer Zugang zu Überzeugungen zum Lehren und Lernen verwendet. Die befragten Lehrkräfte wählten die Metaphern, die ihrer Sicht auf das Lehren und Lernen vor dem Beginn ihrer Lehrtätigkeit und gemäß ihrer aktuellen Lehrtätigkeit entsprachen (Alger, 2009). Zur Auswahl standen lehrerzentrierte Metaphern wie Lehren als Führen, Lehren als Formen der Schülerinnen und Schüler, Lehren als Übertragen von Wissen und lernerzentrierte Metaphern wie Lehren als Lernmittel zur Verfügung stellen und als Teilhabe an einer Lerngemeinschaft sowie die Metapher der Schülerorientierung. Die Ergebnisse zeigten, dass die Mehrheit der Lehrkräfte $(60 \%)$ eine Veränderung in ihren Einsichten wahrnahm. Zudem zeigte sich, dass die Entwicklung der Überzeugungen in Abhängigkeit davon verlief, wie lange die Lehrkräfte tätig waren. Erfahrene Lehrkräfte schienen vor dem Einstieg in den Lehrerberuf ihre Tätigkeit lehrerzentriert (Lehren als Führen, Formen der Schülerinnen und Schüler, Lehren als Übertragen von Wissen) aufzufassen und ihre aktuellen Einsichten in Richtung lernerzentrierter Metaphern (Lehren als Lernmittel zur Verfügung stellen und als Teilhabe an einer Lerngemeinschaft) zu verändern. Auf der anderen Seite zeigten jüngere Lehrkräfte die Tendenz, mit lernerzentrierten Vorstellungen in den Beruf einzusteigen, aber mit der Zeit den Unterricht mehr und mehr lehrerzentriert zu sehen. Insgesamt lassen sich die Ergebnisse so interpretieren, dass während der Lehrerlaufbahn junge Lehrkräfte ihre lernerzentrierte Auffassung von Unterricht aufgeben, dagegen aber Lehrkräfte mit Erfahrung lernerzentriertes 
Unterrichten immer mehr schätzen. Kritisch ist an dieser Stelle anzumerken, dass diese retrospektive Einschätzung eigener Vorstellungen zum Lehren und Lernen eine im Vergleich zur längsschnittlichen Untersuchung weniger valide Methode darstellt.

Zusammenfassend lässt sich festhalten, dass die Ergebnisse bisheriger Forschung eine Veränderung der Lehrerüberzeugungen sowohl während der Lehrerausbildung als auch mit steigender Berufserfahrung erwarten lassen. Lehr-Lern-Überzeugungen von zukünftigen Lehrkräften scheinen am Anfang ihrer Ausbildung eher transmissiv und schülerorientiert zu sein, während die schülerorientierten Aspekte im Verlauf des Studiums schwinden und sich $\mathrm{zu}$ konstruktivistischen Überzeugungen entwickeln. Allerdings wurde auch von gegensätzlichen Veränderungen berichtet, sodass die Frage, welche Überzeugungen Studierende am Anfang des Studiums zeigen und in welche Richtung sich diese im Verlauf des Studiums verändern, nicht ausreichend geklärt ist. Zudem sind die meisten Untersuchungen qualitativ oder stellen gar Fallstudien dar. Sie wenden unterschiedliche methodische Ansätze an und gehen von jeweils anderen Überzeugungen (Metaphern, Konzeptionen) aus, was die Vergleichbarkeit der Ergebnisse erschwert. Längsschnittstudien mit erfahrenen Lehrkräften fehlen bislang und existierende Querschnittstudien beschränken sich auf Vergleiche von zwei Gruppen. Somit besteht deutlicher Forschungsbedarf sowohl an weiteren Untersuchungen von Lehr-Lern-Überzeugungen während der Lehrerausbildung als auch während der Lehrerlaufbahn. Eine Studie, die sich nicht nur auf zwei Zeitpunkte beschränkt, sondern mehrere unterschiedliche Zeitpunkte in der Lehrerlaufbahn analysiert, wäre also gewinnbringend. 


\subsubsection{Einfluss der Lehr-Lern-Überzeugungen auf den Unterricht und die Schülerleistung}

Die aktuelle Forschung lieferte bereits empirische Evidenz dafür, dass Lehrerüberzeugungen sowohl für das Handeln der Lehrkräfte im Unterricht als auch für Schülerleistungen eine Rolle spielen (z.B. Dubberke, 2008; Peterson et al., 1989; Staub \& Stern, 2002). Bereits frühere Fallstudien (Cohen, 1990; Thompson, 1984, 1985) gaben Hinweise darauf, dass Überzeugungen der Lehrkräfte mit ihrem unterrichtlichen Handeln zusammenhängen. Als Erste zeigte die Studie von Peterson et al. (1989) spezifische Korrelationen zwischen den Lehrerüberzeugungen und dem Lehrerhandeln. Im Vergleich zu Lehrkräften, die an das Lernen durch Memorieren von separaten Wissenseinheiten glaubten (traditionelle Auffassung), verwendeten Lehrkräfte, die an die eigene Wissenskonstruktion der Schülerinnen und Schüler glaubten (kognitive Auffassung), mehr Textaufgaben, die zum Verständnis des Problems beitrugen. Die Ergebnisse der Studie von Staub und Stern (2002) mit 496 deutschen Grundschulkindern bestätigten diese Ergebnisse. Konstruktivistische Lehrerüberzeugungen im Mathematikunterricht gingen eher mit dem Einsatz verstehensorientierter Aufgaben und einem größeren Lernzuwachs bei den Kindern einher. Stipek et al. (2001) fanden eine enge Beziehung zwischen den Überzeugungen von 21 amerikanischen Mathematiklehrkräften und ihrer beobachteten Unterrichtspraxis. Berichtet wurde von Zusammenhängen zwischen der Überzeugung, dass Mathematik vorrangig im Beherrschen von Rechenprozeduren und Fakten bestehe, und einem leistungsorientierten Lehrerhandeln als Gegensatz zum lern-/verstehensorientierten Lehrerhandeln. Lehrkräfte mit eher transmissiven Überzeugungen gaben weniger Freiräume für selbstbestimmtes Lernen und legten Wert auf das Vermeiden von Fehlern. Entsprechend zeigten sich hohe negative Korrelationen zwischen der Lehrerüberzeugung, Schülerinnen und Schüler sollen sich im Mathematikunterricht an die Aufgabeanweisungen der Lehrkraft halten, und dem Grad der beobachteten Schülerautonomie beim Problemlösen.

In der Studie von Hartinger et al. (2006) wurden konstruktivistisch orientierte Vorstellungen von Grundschullehrkräften fachübergreifend untersucht sowie bestimmte Unterrichtsmaßnahmen mittels Unterrichtsbeobachtung (Öffnung des Unterrichts in Hinblick auf Entscheidungsmöglichkeiten für Schülerinnen und Schüler und Strukturierung des Unterrichts) und motivationale Auswirkungen auf der Schülerseite durch Schülerauskunft (Selbstbestimmungsempfinden und Interessantheit des Unterrichts) analysiert. Dabei wurde in den Analysen der Schülerwahrnehmung des Unterrichts sowohl für das Geschlecht als auch

für die Klassenstufe kontrolliert, da Schülerinnen ein allgemein höheres Interesse am 
Unterricht aufweisen als Schüler und das Schulinteresse im Laufe der Schulzeit sinkt. An der Studie nahmen 45 Lehrkräfte mit ihren Klassen (1 091 Schülerinnen und Schüler) teil. Die Ergebnisse der Unterrichtsbeobachtung zeigten, dass, verglichen mit Lehrkräften mit transmissiven Vorstellungen, Lehrkräfte mit überwiegend konstruktivistischen Vorstellungen den Schülerinnen und Schülern die meisten Freiräume im Unterricht gewährten, ohne eine geringere Strukturierung des Unterrichts aufzuweisen: Die erhobene Strukturierungsmaßnahme wurde von Lehrkräften mit konstruktivistischen Vorstellungen sogar häufiger durchgeführt. Zudem zeigte sich ein höheres Selbstbestimmungsempfinden bei den Schülerinnen und Schülern von Lehrkräften mit ausgeprägten konstruktivistischen Vorstellungen. Ferner schätzten sie den Unterricht interessanter ein als Schülerinnen und Schüler von Lehrkräften mit transmissiven Vorstellungen. Diese Befunde zur Schülerwahrnehmung sind besonders bemerkenswert, da hier der Effekt der beobachteten Freiräume im Unterricht herausgerechnet wurde und für das Geschlecht und die Klassenstufe kontrolliert wurde. Somit wurde der Effekt der konstruktivistischen Lehrervorstellungen unabhängig von den beobachteten Merkmalen des Unterrichts wie Freiräume sowie unabhängig vom Geschlecht und der Klassenstufe der Schülerinnen und Schüler nachgewiesen.

Im Rahmen der COACTIV-Studie (vgl. Dubberke et al., 2008) wurde mit Hilfe eines längsschnittlichen Designs die Mediationshypothese überprüft, dass Lehrerüberzeugungen im Fach Mathematik, vermittelt über bestimmte Unterrichtsmerkmale, die Schülerleistung beeinflussen. In dieser Studie wurden Daten von 155 Lehrkräften und deren Klassen (3 483 Schülerinnen und Schüler der Klassenstufe 10) verwendet. Lehrerüberzeugungen wurden mittels Zustimmung der Lehrkräfte zu Aussagen über transmissive Überzeugungen gemessen (über das Wesen des Faches Mathematik und über das Lernen und Unterrichten von Mathematik). Die Unterrichtsmerkmale Effizienz der Klassenführung, kognitive Aktivierung und konstruktive Unterstützung wurden mittels der Schülereinschätzung erhoben. Dabei gingen Klassenmittelwerte in die Analysen ein, um die zwischen den Klassen bestehenden Leistungsunterschiede auf Merkmale des Unterrichts und Lehrerüberzeugungen der entsprechenden Lehrkraft zurückzuführen. In den Analysen wurde sowohl für soziale und berufliche Hintergründe der Lehrkräfte als auch für wichtige Schülermerkmale, wie mathematische Kompetenzen am Ende der Klassestufe 9, kognitive Grundfähigkeit, Lesekompetenz, Migrationsstatus und Bildungshintergrund der Eltern kontrolliert. Das Ergebnis der Studie demonstrierte die Bedeutsamkeit der Lehrerüberzeugungen für die Unterrichtsgestaltung und die Mathematikleistung: Schülerinnen und Schüler von Lehrkräften 
mit stark transmissiven Überzeugungen gaben an, weniger kognitiv herausgefordert und weniger in ihrem Lernprozess unterstützt zu werden. Im Hinblick auf die Mediationsannahme zeigte sich vor allem eine vermittelnde Rolle der kognitiven Aktivierung: Lehrkräfte mit stark ausgeprägten transmissiven Überzeugungen gestalteten ihren Unterricht weniger kognitiv herausfordernd, was sich nachteilig für den Lernerfolg der Schülerinnen und Schüler erwies (Dubberke et al., 2008).

Diese Untersuchungen belegen die Bedeutung der Lehrerüberzeugungen für die Unterrichtsgestaltung und die Schülerleistung. Die genannten Studien befassten sich jedoch mit nicht mehr als zwei Überzeugungen, sie fokussierten transmissive und konstruktivistische Überzeugungen. Andere Sichtweisen auf das Lehren und Lernen, beispielsweise partizipatorische und schülerorientierte Überzeugungen, wurden nicht beachtet. Hartinger et al. (2006) zum Beispiel sprachen selbst davon, dass das stärkere Interesse am Unterricht der Schülerinnen und Schüler von Lehrkräften mit konstruktivistischen Überzeugungen auch durch einen möglicherweise anderen persönlichen Umgang der Lehrkräfte mit ihren Schülerinnen und Schülern erklärt werden könnte (vgl. Deci \& Ryan, 1993). Der hier angesprochene persönliche Umgang lässt konzeptuell den Aspekt der Schülerorientierung erkennen (Alger, 2009; Pratt et al., 2001). Es wäre also denkbar, eine Lehrerüberzeugung identifizieren zu können, die die Schülerpersönlichkeit in den Vordergrund stellt, welche die Unterschiede im Schülerinteresse am Unterricht und andere Merkmale des Schülerlernens besser erklärt als die konstruktivistische Überzeugung. Eine ähnliche Annahme kann gemacht werden, wenn man Ergebnisse der Studien aus der Motivationsforschung hinzuzieht. Diese demonstrieren weniger Schülerbedenken bezüglich ihrer Leistung, wenn Lehrkräfte die Betonung auf Kraftinvestition beim Lernen und auf Verstehen anstatt auf bloßes Faktenlernen legen und eine Lernatmosphäre schaffen, die die Schülerinnen und Schüler dazu anregt, Fehler zu riskieren. Fehler werden nämlich als Teil des Lernprozesses behandelt, die das Verständnis überhaupt erst ermöglichen (siehe Überblicksartikel bei Stipek 1996b, 1998).

Schülerorientierung und auch Partizipation sind die beiden Überzeugungen zum Lehren und Lernen, die, wie oben erwähnt, fast ausschließlich qualitativ untersucht wurden: Partizipative Vorstellungen wurden zum Beispiel bei Alger (2009) als teaching as engaging in community und bei Martínez et al. (2001) als situative/sociohistoric view dargestellt. Zudem wurden diese Überzeugungen in den einzelnen Studien unterschiedlich operationalisiert: Alger (2009) zum Beispiel analysierte die Lehr-Lern-Überzeugung mithilfe von Metaphern des Lehrens und Lernens. Für „Engagement in einer Gemeinschaft“ (engl. engaging in the community) wird in ihrer Studie die Metapher verwendet: „I am a part of a 
community that is building a house. We collectively decided that we need a house and then we design and build it together" (Alger, 2009; S. 744). Pratt et al. (2001) präsentieren mit ihrem TPI einen Fragebogen, mit dessen Hilfe sie auch die Perspektive Partizipation (nach Pratt et al., 2001: engl. apprenticeship) und Schülerorientierung analysierten. Allerdings wurden für TPI keine Validierungsstudien publiziert, und auch der Versuch einer Übersetzung ins Deutsche blieb erfolglos (Hanke \& Schwarz, 2011). Somit fehlt es bis heute an einer einheitlichen Operationalisierung der partizipatorischen und schülerorientierten Überzeugungen, sodass es keine zufriedenstellende Möglichkeit zur ökonomischen und validen Erfassung dieser Überzeugungen gibt.

Schlussfolgernd kann wiederholt betont werden, dass der Einfluss von Lehrerüberzeugungen auf das Unterrichtverhalten und die Schülerleistung nur für transmissive und konstruktivistische Überzeugungen belegt werden konnte (Fennema et al., 1990; Dubberke et al, 2008; Peterson et al., 1989; Staub \& Stern, 2002). So bleibt die Frage offen, welche Bedeutung andere Lehrerüberzeugungen in schulischen Lehr-Lern-Kontexten tragen können: Wie gestalten Lehrkräfte mit partizipatorischen Überzeugungen ihren Unterricht? Welche Wirkung hat die Überzeugung Schülerorientierung auf das Lernen von Schülerinnen und Schülern? Inwieweit beeinflussen diese Überzeugungen von Lehrkräften die Schülerleistung? Bevor diesen Fragen nachgegangen werden kann, müssen die bislang wenig untersuchten Überzeugungen Partizipation und Schülerorientierung empirisch messbar gemacht werden. Daher besteht Forschungsbedarf in der Entwicklung eines Messinstruments zur Erfassung von Partizipation und Schülerorientierung. Diesem Forschungsbedarf wird in der vorliegenden Arbeit nachgegangen. Im ersten Schritt werden verschiedene Facetten der vier Überzeugungen in den Interviews beleuchtet, um im nächsten Schritt einen Fragebogen zu entwickeln, der diese Überzeugungen reliabel und valide erfasst. Somit ist der methodische Zugang in den Studien der vorliegenden Arbeit sowohl qualitativ als auch quantitativ. Einerseits klären die Interviews auf, inwieweit die hier thematisierten Überzeugungen in den Lehr-Lern-Ansichten der Lehrkräfte präsent sind und andererseits ermöglicht die Entwicklung des Fragebogens die vier genannten Überzeugungen in einem ökonomischen Format messbar zu machen. 


\subsection{Erfassung von Lehr-Lern-Überzeugungen}

\subsubsection{Methodische Zugänge zu Lehr-Lern-Überzeugungen}

Welche Überzeugungen eine Lehrkraft von Lehren und Lernen hat, kann auf zwei Wegen erforscht werden. Zunächst können Lehrkräfte zu dieser Thematik befragt werden. Weiterhin bietet die Erhebungsmethode mittels Fragebogen die Möglichkeit zeitökonomisch größere Personenzahlen zu erreichen. Die Herausforderung besteht hierbei darin, handlungswirksame Überzeugungen oder zumindest solche, die handlungswirksam werden können, zu messen. In der bisherigen Forschung wurden einige methodische Zugänge zu solchen handlungswirksamen Überzeugungen vorgestellt. So werden beim Stimulated Recall Interview nach Calderhead (1981) videografierte Sequenzen des Unterrichts der zu befragenden Lehrkraft verwendet. Dabei wird die Lehrkraft zu ausgewählten Stellen des Unterrichtsablaufs gefragt, was ,ihr durch den Kopf gegangen ist“. Dieses Vorgehen wird aufgrund der nachträglichen Rekonstruktion kognitiver Prozesse aus der Entscheidungssituation kritisiert, da Gedanken, die man in einem bestimmten Moment hatte, im Nachhinein nur schwer erinnert werden (Nisbett \& Wilson, 1977). Die von Bromme (1981) beschriebene Methode des „lauten Denkens“ wäre interessant für die Erfassung der Überlegungsvorgänge während eines Handlungsvollzugs. Allerdings ist sie kaum vorstellbar in einer Unterrichtssituation. Interviews mit „fremden“ Unterrichtsszenen sind weniger aufwendig als das Stimulated Recall Interview, weil sie eine geringere Videografie beanspruchen. Das Vorgehen birgt aber eine Reihe von Nachteilen, so zum Beispiel, dass die Auswahl der Unterrichtsszenen ausreichend begründet sein muss. Zudem stellt die präsentierte Unterrichtsszene eine Darstellung des Verhaltens einer anderen Person dar, sodass nur Annahmen über mögliche Handlungen getroffen werden können. Eine gute Alternative stellt die Methode nach White und Gunstone (1992) dar, bei welcher eine selbst angefertigte Zeichnung als Impuls zu einem Interview eingesetzt wird. Die zu befragende Person wird vor dem Interview gebeten, eine Zeichnung zum klärenden Begriff anzufertigen. Durch den Prozess des Zeichnens werden subjektive Erinnerungen aktiviert (White \& Gunstone, 1992). Verglichen mit der Videografie ermöglicht diese Methode einen ökonomischen Zugang zu Lehrerüberzeugungen. Sie eignet sich gut dazu, die subjektiv gesammelten Erfahrungen einer Person zum Lehren und Lernen und die damit verbundenen Situationen für das Interview zugänglich zu machen. Diese Methode wurde im Rahmen der ersten Studie der vorliegenden Arbeit verwendet, um subjektive Vorstellungen der 
Interviewten zu aktivieren und so im Interview die persönlichen Überzeugungen zum Lehren und Lernen erfahren zu können.

Eine weitere Methode, mit der unterschiedlich große Stichproben zeitsparend befragt werden können, stellt das Fragebogenverfahren dar. Oftmals wird der Einsatz von Fragebögen kritisiert, denn Fragebögen „besitzen nicht die Flexibilität, die notwendig ist, um situationsbezogene Vorstellungen so auszuloten, dass der Zusammenhang zwischen erklärten Handlungszielen und darauf abgestimmten Handlungen sichtbar wird“ (Fischler, 2001, S. 106). Durch die Vorgabe von Begriffen ist ein Konsens zwischen dem Verständnis des Begriffs auf der Seite des Befragten und des Forschers nicht problemfrei, sodass gewisse Missverständnisse in den Antworten der Befragten auftreten können. Durch die vorgefertigten Fragen können keine neuen Ideen erforscht werden. Deshalb ist bei der Wahl des methodischen Zugangs die Überlegung anzustellen, wie neuartig der Gegenstand der Untersuchung ist. Will man neue Informationen erhalten, eignen sich Interviews. Ist der Gegenstand bereits dargestellt und empirisch untersucht - die Ergebnisse müssen aber noch an einer größeren Stichprobe abgesichert werden - so empfiehlt sich die Fragebogenmethode. Da beide Methoden Vor- und Nachteile aufweisen, kann ein multimethodisches Vorgehen, bei dem sowohl Fragebögen als auch Interviews verwendet werden, die Schwächen der einen Vorgehensweise durch die Stärken der anderen ausgleichen. In einem Forschungsfeld, das sowohl bekannte als auch neue, weniger erforschte Konstrukte beinhaltet, wäre es optimal, beide Methoden - Interviews und Fragebögen - einsetzen zu können. In der vorliegenden Arbeit wird daher auf beide Methoden zurückgegriffen, indem in der ersten Studie mithilfe von Interviews mit angehenden und berufserfahrenen Lehrkräften ihre Überzeugungen zum Lehren und Lernen qualitativ analysiert und in der zweiten Studie diese Überzeugungen mithilfe eines Fragebogens quantitativ gemessen werden.

\subsubsection{Messinstrumente zur Erfassung von Lehr-Lern-Überüberzeugungen}

In diesem Kapitel sollen existierende Fragebögen zur Erhebung von Überzeugungen zum Lehren und Lernen präsentiert werden.

Die meisten in der empirischen Forschung bereits existierenden Instrumente zur Erfassung von Lehrerüberzeugungen zum Lehren und Lernen analysierten zwei Überzeugungen: transmissive und konstruktivistische Überzeugungen, die als bipolare Konstrukte konzipiert werden. Diese beiden Überzeugungen fanden Zugang in größere international angelegte Befragungen wie dem Teaching and Learning International Survey 
(TALIS) der OECD (2009). Im TALIS wird unter transmissiver Überzeugung die Rolle des Lehrers verstanden, klar strukturierte Lerninhalte zu präsentieren und die richtige Lösung zu erklären, den Schülerinnen und Schülern klare und lösbare Aufgaben zu erteilen sowie für ein ruhiges und konzentriertes Arbeiten im Klassenraum zu sorgen (OECD, 2009, S. 92). Im Gegensatz dazu werden Lerner nach der konstruktivistischen Überzeugung nicht als passive, sondern als aktive Teilnehmer im Prozess der Wissensaneignung aufgefasst. Die Aufgabe des Lehrers wird in der Unterstützung des Schülerlernens gesehen, indem Schülerinnen und Schülern die Möglichkeit gegeben wird, sich ihre eigenen Lösungen für Aufgaben zu erarbeiten. Die Entwicklung von Denkprozessen ist somit wichtiger als die Aneignung von Faktenwissen (OECD, 2009, S. 92). Im TALIS werden beide Überzeugungen anhand von jeweils vier Items auf einer vierstufigen Likert-Skala erhoben. Ein Beispielitem für die Transmission ist: „Effective/good teachers demonstrate the correct way to solve a problem”; für die Konstruktion: "My role as a teacher is to facilitate students' own inquiry".

Ebenso wurde in der deutschsprachigen Studie von Staub und Stern (2002) das Überzeugungspaar gegenübergestellt: direct transmission als die auf assoziationistischer Theorie basierende Überzeugung auf der einen Seite und cognitive construction als die auf der konstruktivistischen Theorie basierende Vorstellung von Lehren und Lernen. Die Annahme der Bipolarität beider Überzeugungen wird der Konstruktion des Fragebogens zugrunde gelegt, indem die Items der Skalen jeweils zur Hälfte positiv formuliert und zur anderen Hälfte negativ formuliert sind, wobei die negativen Items inhaltlich die transmissive Überzeugung wiedergeben. So geben die erreichten Werte im Fragebogen die Zustimmung zur konstruktivistischen Überzeugung wieder, was die gleichzeitige Ablehnung der transmissiven Überzeugung bedeutet. Insgesamt umfasst das Instrument vier Skalen mit jeweils zwölf Items. Das Instrument der Studie von Staub und Stern (2002) diente einer Reihe nachfolgender Untersuchungen als Orientierung. Dabei handelt es sich um eine deutsche Übersetzung des Fragebogens von Fennema et al. (1990), der auf drei Skalen aus dem Instrument nach Peterson et al. (1989) und auf einer Skala aus einem verwandten Fragebogen nach Cobb, Wood, Yackel, Nicholls, Wheatley, Trigatti et al. (1991) basiert. Der Fragebogen umfasst vier Skalen:

1) die Rolle des Lerners (Beispielitem für ein positives Item: „Schüler lernen Mathematik am besten, indem sie selbst Wege zur Lösung von einfachen Textaufgaben entdecken“; Beispielitem für ein negatives Item: „Schüler lernen Mathematik am besten, indem sie den Erklärungen der Lehrerin oder des Lehrers folgen“), 
2) Relation zwischen Fähigkeiten, dem Verständnis und dem Problemlösen (Beispielitem: „Im Unterricht sollen Schüler bereits einfache Textaufgaben erhalten, bevor viel Zeit auf das Üben von Rechenprozeduren verwendet wird“),

3) Sozialkonstruktivismus (Beispielitem: „Anhand geeigneter Materialien können Schüler selbst Rechenprozeduren entwickeln“) und

4) die Rolle des Lehrers (Beispielitem für ein positives Item: „Man sollte Schülern erlauben, sich eigene Wege zur Lösung von einfachen Textaufgaben auszudenken, bevor die Lehrperson vorführt, wie diese zu lösen sind“; Beispielitem für ein negatives Item: „Am besten lehrt man Schülern das Lösen von Textaufgabe, indem man nicht verschiedene Arten von Textaufgaben zugleich behandelt, sondern sich jeweils auf eine Art beschränkt“.

In der COACTIV-Studie von Baumert und Kollegen (z.B. Kunter et al., 2011) wurden transmissive und konstruktivistische Überzeugungen anhand einer Reihe von Skalen erhoben, „Überzeugungen über das Wesen mathematischen Wissens“, „Überzeugungen über das Lernen und Unterrichten von Mathematik“ und „Zielvorstellungen zum Unterricht“. „Lehrkräfte sollen Schüler/innen ermutigen, ihre eigenen Lösungswege für Mathematikaufgaben zu suchen, selbst wenn diese ineffizient sind“ - so lautet ein Item für die konstruktivistische Überzeugung der Skala „Selbstständiges und verständnisvolles diskursives Lernen“. „Am besten lernen Schüler/innen Mathematik aus Darstellungen und Erklärungen ihrer Lehrkraft" stellt ein Beispielitem für die transmissive Überzeugung der Skala „Rezeptives Lernen durch Beispiele und Vormachen“ dar.

In der PaLea-Studie zur Entwicklung berufsbezogener Merkmale von Studierenden im Verlauf des Lehramtsstudiums (Bauer et al., 2009) wurden unter anderem subjektive Überzeugungen und Wertehaltungen erhoben. Die Skala zur Messung subjektiver Lern/Lehrtheorien bestand aus 18 Lern- und 13 Lehrbegriffen, welche die Studierenden nach deren Ähnlichkeit ihrer Vorstellung von Lernen und Lehren einschätzten. Die Lernbegriffsskala bestand aus drei Subskalen „Pauken“ (Beispielitem: „... sich anstrengen“), „Explorieren“ (Beispielitem: „... sich entwickeln“) und „Verstehen“ (Beispielitem: „.. begreifen“). Die Lehrbegriffsskala umfasste zwei Subskalen „Instruktionsorientiert“ (Beispielitem: „... kontrollieren“) und „Lernbegleitungsorientiert“ (Beispielitem: „,.. Interesse wecken“).

Im Fragebogen „Approaches to Teaching Inventory“ (ATI) nach Trigwell und Prosser (2004) werden Lehransätze der Hochschullehrkräfte mit Hilfe von zwei Skalen ,information transmission/ teacher focused view of teaching“ (ITTF) und „conceptual change/ studentfocused view of teaching“ (CCSF) mit insgesamt 16 Items erhoben. Ein Beispielitem für die Skala ITTF lautet: „I feel it is important to present a lot of facts to students so that they know 
what they have to learn for this subject”. Ein Beispielitem für die Skala CCSF „I feel that it is better for students in this subject to generate their own notes rather than always copy mine" (Trigwell \& Prosser, 2004, S. 424).

Das Instrument der Studie von Norton et al. (2005) setzt die Idee des ATI fort, dass sich Überzeugungen in Strategien und Ziele trennen lassen, und versucht zwischen Überzeugungen und Zielen zu unterscheiden, indem Subskalen für beide Aspekte entwickelt wurden. Es wurden 34 Items generiert, die insgesamt neun Subskalen repräsentieren, von welchen sich die Subskalen „problem solving“, „more interactive teaching“, ,facilitative teaching“, „pastoral interest" und „,motivator of students“ zu der Kategorie höherer Ordnung „learning facilitation“ zusammensetzen und die vier Subskalen „training for specific job“, „greater use of media“, ,imparting information“ und „,knowledge of subject“ $\mathrm{zu}$, ,knowledge transmission“ zusammengefasst werden. Ein Beispielitem der Skala „learning facilitation“ lautet: „Teaching is about providing an environment in which students are encouraged to do the learning themselves“, ein Beispielitem für „knowledge transmission” heißt: „A good lecturer is one whose main role is to impart information to his/her students" (Norton et al., 2005, S. 566).

Die deutschsprachige Übersetzung des Instruments ATI wurde im Rahmen des Dissertationsprojektes von Lübeck (2009) eingesetzt. Ein Beispielitem für die Skala „,information transmission“ lautet „Es ist wichtig, den Studierenden möglichst viele Fakten zu präsentieren, damit sie wissen, was sie für dieses Fach lernen müssen“ und ein Beispielitem für „,conceptual change“ lautet: „Ich ermutige die Studierenden, ihr bestehendes Wissen so zu rekonstruieren, dass sie sich die Inhalte auch unter Berücksichtigung neuer Denkweisen erschließen können“ (Lübeck, 2009).

Die empirische Forschung konzentrierte sich bislang auf die zwei Überzeugungen knowledge transmission und cognitive construction (Kunter et al., 2011; Peterson et al., 1989; Staub \& Stern, 2002). Analysen weiterer Überzeugungen sowie Erhebungsinstrumente zur Messung von Überzeugungen wie Partizipation oder Schülerorientierung fehlen bis heute. Es existierten lediglich vereinzelt Versuche, die Überzeugung Schülerorientierung, in der Bezeichnung nach Pratt et al. (2001) nurturing perspective, mithilfe des Fragebogens „Teaching Perspective Inventory“ (TPI) zu erheben (Pratt et al., 2001). Verglichen mit den oben genannten Instrumenten stellt das TPI eine Ausnahme dar, weil es sich nicht auf zwei Überzeugungen beschränkt. Mit dem TPI können fünf Perspektiven gemessen werden: 
1) transmission, welche das effektive Lehren mit der Beherrschung von Faktenwissen gleichsetzt (Beispielitem: „I expect people to master a lot of information related to the subject");

2) apprenticeship, wonach Lernen einen Prozess der Enkulturation eines neuen Lerners in ein Set sozialer Normen und Arbeitsweisen darstellt (Beispielitem: „The best learning comes from working alongside good practitioners";

3) developmental, die besagt, dass Lehren aus der Sicht des Lerners geplant und organisiert sein muss (Beispielitem: „Teaching should build upon what people already know");

4) nurturing, welche das Ziel des Lehrens in der Motivierung von Schülerinnen und Schülern durch Herstellen einer angstfreien Atmosphäre sieht (Beispielitem: „In learning, people's effort should be rewarded as much as achievement”) sowie

5) social reform, wonach Lehren der Verbesserung der Gesellschaft dient (Beispielitem: „I expect people to be committed to changing our society”). Eine deutsche Übersetzung wurde in der Arbeitsgruppe von Hanke am Institut für Erziehungswissenschaften der Universität Freiburg angestrebt. In mehreren Übersetzungsstufen wurden Items auf Deutsch entwickelt: Ein Beispielitem für nurturing ist: „Während meiner Lehre hat der Aufbau von Selbstvertrauen bei den Lernenden Priorität“. Die Übersetzung des TPI verlief bislang wenig zufriedenstellend, sodass Hanke und Schwarz (2011) die Entwicklung eines deutschen Fragebogens empfehlen.

Zusammenfassend lässt sich festhalten, dass in der bisherigen empirischen Forschung zu Lehrerüberzeugungen besonders den Überzeugungen Transmission und Konstruktion Aufmerksamkeit geschenkt worden ist. Dies führte dazu, dass einige Instrumente $\mathrm{zu}$ deren Messung entwickelt worden sind. Weitere Überzeugungen wie Partizipation und Schülerorientierung wurden bisher in der quantitativen Forschung vernachlässigt, sodass es an Messinstrumenten für diese Überzeugungen fehlt.

\subsubsection{Dimensionalität der Überzeugungen}

Die Frage nach der Dimensionalität von Überzeugungen wurde im vorherigen Kapitel bereits angesprochen, hier soll sie gesondert behandelt werden. Da sich die meisten bisherigen Instrumente auf zwei Überzeugungen - Transmission und Konstruktion - beziehen, wird in diesem Kapitel die Frage nach der Dimensionalität anhand dieser zwei Überzeugungen diskutiert. In der bisherigen Forschung wurde der Dimensionalität der Überzeugungen nicht 
immer ausreichend Beachtung geschenkt (Lübeck, 2009). Entscheidend wird sie aber, wenn man die Beziehung der Überzeugungen zueinander definiert und vor allem existierende Instrumente zur Diagnose von Lehr-Lern-Überzeugungen bei Lehrkräften verwenden möchte (Lübeck, 2009). Handelt es sich bei Überzeugungen um ein eindimensionales Konstrukt mit zwei Endpolen? Wenn man die Bipolarität der zwei Überzeugungen annimmt, hieße die Frage, ob eine Person entweder die eine oder die andere Überzeugung hat: knowledge transmission ODER cognitive construction. Handelt es sich aber um zwei Konstrukte: knowledge transmission UND cognitive construction mit dementsprechend unterschiedlichen Ausprägungen in beiden Überzeugungen, würde man sich fragen, ob und wie sich diese zueinander verhalten: Sind sie distinkt, also klar und deutlich voneinander abgrenzbar und unkorreliert? Schließen sie sich gegenseitig aus, lässt sich also eine negative Korrelation zwischen den zwei Überzeugungen finden? In diesem Fall würde man zum Beispiel bei niedrigen Werten in der Transmission hohe Werte in der Überzeugung Konstruktion erwarten.

In der Literatur werden unterschiedliche Überzeugungen nicht immer trennscharf verwendet, sodass oft unklar bleibt, ob einzelne Überzeugungen ein- oder mehrdimensional konzipiert und wie die Annahmen zur Abgrenzbarkeit verschiedener Kategorien getroffen werden. Bei der theoretischen Auseinandersetzung mit dieser Frage wird oft die Eindimensionalität angenommen, wie zum Beispiel die Eindimensionalitätsannahme der Lehrkonzeptionen mit den Extrempositionen der dozentenzentrierten Informationsvermittlung und studierendenorientierten Lernunterstützung (Winteler, 2001). Auch in dem in der Studie von Staub und Stern (2002) verwendeten Fragebogen nach Peterson et al. (1989) wird die Bipolarität der Überzeugungen Transmission und Konstruktion angenommen. Die positiven Items geben konstruktivistische Einstellungen wieder und die negativen Items transmissive Einsichten, sodass die Zustimmung zu der zweiten Gruppe einen niedrigeren Wert für Konstruktion bedeutet. Ebenso stellen Kember und Kwan (2000) die verschiedenen Lehransätze auf einem Kontinuum mit den Endpolen „inhaltsorientiert“ versus „lernorientiert“ dar. Dabei werden als Übergangskonzeptionen zwischen den beiden Extrempolen fünf idealtypische Lehrkonzeptionen dargestellt: von Wissensvermittlung, über Vermittlung strukturierten Wissens, Interaktion Lehrende/r-Studierende/r und Unterstützung des Verständnisses bis hin zur Veränderung von Wissensstrukturen.

Empirische Befunde dafür, dass Personen nicht nur eine Lehr-, sondern mehrere, auch inhaltlich gegensätzlich postulierte Lehrorientierungen haben können, stellen die Annahme der Eindimensionalität in Frage (Aguirre \& Speer, 2000; Meyer \& Eley, 2003; Murray \& MacDonald, 1997). So sprechen Trigwell, Prosser und Ginns (2005) im Zusammenhang mit 
Lehransätzen von zwei unabhängigen Dimensionen: lehrerzentriert und schülerzentriert. Dementsprechend können Lehrkräfte sowohl in der schülerzentrierten als auch in der lehrerzentrierten Lehrkonzeption hohe Werte erzielen. Eine ähnliche, pluralistische Position in dieser Frage bezieht Pratt (2002), indem er sich explizit gegen einen „konstruktivistischen Dogmatismus“ ausspricht, denn jede der fünf von ihm postulierten Lehrperspektiven trägt das Potenzial zu einem guten Unterricht. Der Möglichkeit, dass eine Person entweder die eine oder die andere Perspektive bevorzugt, wird Rechnung getragen, indem die Präferenz bzw. die Ablehnung einer Perspektive durch die Befragten als deren „dominierende“ versus „rezessive“ Perspektive bestimmt wird (Pratt et al., 2001).

Die meisten Studien setzten mehrere Skalen ein, was auf die Annahme der Mehrdimensionalität der Überzeugungen schließen lässt. Explizite Hinweise darauf finden sich allerdings nicht in allen dieser Studien. Bezüglich der Frage der Beziehung zwischen den zwei Überzeugungen Transmission und Konstruktion wurde im TALIS von deren gegensätzlichem Charakter ausgegangen, und es wurden negative Zusammenhänge oder zumindest Null-Korrelationen erwartet (OECD, 2009). Die Ergebnisse der teilnehmenden Staaten zeigten widersprüchliche Befunde. Mal ergaben sich negative Korrelationen zwischen den zwei Überzeugungen, wie zum Beispiel bei Lehrkräften aus Australien, Österreich und Island, welche bei hohen Werten für Konstruktion niedrigere Werte in Transmission aufweisen und umgekehrt, wobei allgemein die erste Perspektive präferiert wurde, und manchmal zeigten sich mittelhohe positive Korrelationen, wie zum Beispiel bei Lehrkräfen aus Asien sowie Zentral- und Südamerika (z.B. Brasilien, Mexiko), welche die beiden Überzeugungen weniger widersprüchlich empfanden und ihre Präferenz für beide in etwa gleich hoch einschätzten, genauso wie Lehrkräfte aus Osteuropa (z.B. Bulgarien, Litauen, Polen).

Für die vorliegende Arbeit erscheint somit die Schlussfolgerung denkbar, dass Lehrerüberzeugungen ein mehrdimensionales Konstrukt darstellen: Eine Lehrkraft kann teilweise konstruktivistische und teilweise transmissive Überzeugungen zum Lehren und Lernen haben. Somit soll für die vorliegende Arbeit der Ansatz von Pratt et al. (2001) als Orientierung dienen, in welchem nach Präferenzen für bestimmte Perspektiven gefragt wurde und es wird in der ersten Studie der vorliegenden Arbeit für jeden Befragten eine dominierende Überzeugung ermittelt. 


\subsection{Epistemologische Überzeugungen}

\subsubsection{Epistemologische Überzeugungen der Lehrkräfte. Eine Begriffsklärung}

Das Konstrukt epistemologischer Überzeugungen lässt sich unter dem Oberbegriff Lehrerüberzeugungen einordnen. Lehrerüberzeugungen werden als Annahmen über Charakteristika von Objekten (Nisbett \& Ross, 1980) verstanden oder als mentale Repräsentationen gesammelter Erfahrungen (Sigel, 19985) oder gegebener Realität definiert (Harvey, 1986). Dabei umfasst der Oberbegriff Lehrerüberzeugungen eine Vielfalt von Aspekten, über die subjektive Annahmen gebildet werden: Es kann sich um Überzeugungen $\mathrm{zu}$ Bildung handeln, um Erwartungen hinsichtlich bestimmter Schülerleistungen oder um Überzeugungen $\mathrm{zu}$ den Ursachen von Lehrer- und Schülerleistung. Epistemologische Überzeugungen stellen als Ansichten über das Wissen, seine Struktur und Genese (Duell \& Schommer-Aikins, 2001; Hofer, 2000, 2001; Hofer \& Pintrich, 1997; Howard et al., 2000; Schommer, 1990, 1994b) einen Bereich aus dem Komplex der Lehrerüberzeugungen dar.

Die zentrale Annahme bei epistemologischen Überzeugungen, ähnlich der Annahme bei lehr-/lerntheoretischen Überzeugungen, lautet, dass diese intuitiven Theorien die Art der Begegnung mit der erkennbaren Welt vorstrukturieren. Ursprünglich stammt das Konstrukt aus der Philosophie und befasste sich mit der Frage nach der Rechtfertigung menschlichen Wissens. In den 1970er Jahren fand das Konzept Eingang in die psychologische Forschung und die Bildungsforschung.

\subsubsection{Modelle epistemologischer Überzeugungen}

Perry (1970) befasste sich als einer der Ersten mit der Entwicklung epistemologischer Überzeugungen von Studenten während der universitären Ausbildung. Sein Stufenmodell unterscheidet ein dualistisches Wissensverständnis mit der Vorstellung von der Wirklichkeit als eindeutig „richtig“ oder „falsch“, ein multiplizistisches Wissensverständnis, das verschiedene Standpunkte anerkennt und eine prinzipielle Unsicherheit von Wissen akzeptiert, ein relativistisches Wissensverständnis mit der Einsicht in die Subjektivität und Abhängigkeit von Erkenntnisverifizierung gewählter Analysemethoden sowie die Stufe der Verbindlichkeit zu einer relativistischen Vorstellung (engl. commitment within relativist), bei der trotz einer relativistischen Sicht Werteentscheidungen getroffen und eigene Positionen bezogen werden. Die vier Stufen werden sukzessiv voneinander abgelöst. 
Perrys Arbeit wurde in der Entwicklungspsychologie in mehreren voneinander unabhängigen Studien fortgeführt und zum Teil bestätigt - so zum Beispiel in der Studie von Magolda (1996) mit 70 Studierenden der Bildungswissenschaften. In dieser Studie wurde die synchrone Entwicklung epistemologischer Überzeugungen bestätigt und ähnliche Entwicklungsstufen identifiziert: von absolutem Wissen über Übergangswissen und unabhängigem Wissen zu kontextuellem Wissen hin. Die Entwicklung epistemologischer Überzeugungen ist demnach vom Alter und Bildungshintergrund abhängig: Kinder werden als Realisten, Jugendliche als Absolutisten, junge Erwachsene als Multiplizisten (engl. musliplist) und Erwachsene als Evaluisten beschrieben (Kuhn, Cheney \& Weinstock, 2000). Dabei können Personen die Evaluisten-Stufe in einer Disziplin erreichen, in einer anderen jedoch noch auf dem Niveau von Absolutisten oder Multiplizisten verbleiben (Kuhn et al., 2000; Kuhn \& Park, 2005).

Im Gegensatz zur entwicklungspsychologischen Forschung, bei der Unterschiede in den epistemologischen Überzeugungen als persönliche Entwicklung entlang verschiedener Stufen erklärt werden, beschreibt der pädagogisch-psychologische Ansatz Ansichten über das Wissen mithilfe verschiedener, voneinander unabhängiger Dimensionen (Schommer, 1990, 1993, 1998). Schommer nimmt an, dass sich das System epistemologischer Überzeugungen nicht als ein unidimensionales Konstrukt operationalisieren lässt, denn „epistemological beliefs are far too complex to be captured in a single dimension“" (Schommer, Crouse \& Rhodes, 1992, S. 436). Sie unterscheidet fünf Dimensionen, nach denen epistemologische Überzeugungen analysiert werden können: a) Struktur, b) Sicherheit und c) Quelle des Wissens. Diese drei lassen sich auf Perrys Arbeiten zurückführen. Zwei zusätzliche Dimensionen wurden aus den Arbeiten Dwecks zu Geschwindigkeit und Kontrolle über Lernen (Dweck \& Legget, 1988) und aus den Arbeiten Schoenfelds zu Überzeugungen über Mathematik (Schoenfeld, 1983) übernommen. Diese zwei Dimensionen sind: d) Lernfähigkeit und e) Lerngeschwindigkeit (Schommer, 1998). Jede der fünf Dimensionen kann entlang eines Kontinuums von naiven $\mathrm{zu}$ fortgeschrittenen (engl. sophisticated) Epistemologien variieren. Zum Beispiel wäre in der Dimension „Struktur des Wissens“ eine naive Überzeugung die Vorstellung vom Wissen als einfach und in der Dimension „Sicherheit" eine Vorstellung des Wissens als klar, sicher und genau bestimmt. Bei den Dimensionen des Lernens wäre ein Glauben an eine angeborene unveränderbare Lernfähigkeit naiv, sodass man entweder schnell lernt oder gar nicht lernen kann. Eine fortgeschrittene Vorstellung wäre dagegen: Das Wissen ist komplex, unsicher, vorläufig gültig, schrittweise erlern- und erfahrbar, vom einzelnen Lerner konstruierbar und in Diskursen verhandelbar (Howard et al., 
2000; Schommer, 1994b). Während Personen mit fortgeschrittenen epistemologischen Überzeugungen glauben, dass es noch zu entdeckendes Wissen gibt und nur eine begrenzte Menge an Wissen gesichert ist, haben Personen mit naiven epistemologischen Überzeugungen die Vorstellung, dass das Wissen als bereits entdeckt betrachtet werden kann. Daher unterstützen fortgeschrittene epistemologische Überzeugungen flexibles Denken und die Fähigkeit zum Überdenken von alten und das Annehmen von neuen Ideen (SchommerAikins, 2002).

Hofer und Pintrich (1997) stellen einen Ansatz dar, der den entwicklungspsychologischen Ansatz (Perry, 1970) und das Modell unabhängiger Dimensionen epistemologischer Überzeugungen (Schommer, 1994b) zu vereinen versucht. Dabei werden lediglich die Vorstellung über die Struktur des Wissens sowie die über die Struktur der Wissenserzeugung als Kerndimensionen epistemologischer Überzeugungen aufgefasst. Sicherheit des Wissens und seine Komplexität stellen Dimensionen der Struktur des Wissens dar und der Entstehungszusammenhang/die Quelle der Wissenserzeugung sowie der Rechtfertigungszusammenhang (engl. justification for knowing) die Dimensionen der Wissenserzeugung. Empirisch bestätigt wurden die Faktoren Sicherheit und Komplexität von Wissen sowie der Rechtfertigungszusammenhang der Wissenserzeugung (Hofer, 2000).

Trautwein und Lüdtke (2004) folgten der Auffassung von Hofer und Pintrich (1997), dass die Struktur des Wissens die Kerndimension epistemologischer Überzeugungen darstellt. Analysen ihres eigens erstellten Itempools zu wissenschaftlicher Erkenntnis, in den Items von Schommer (1993) und Hofer (2000) in einer Adaptation durch Schiefele, Moschner und Hustegge (2002) übernommen wurden, zeigten zwei Dimensionen epistemologischer Überzeugungen: Dualismus und Relativismus. Dualismus gibt eine Einstellung wieder, die von Vertrauen in wissenschaftliche Erkenntnisse sowie in die Fähigkeit der Wissenschaft, eines Tages alles erklären zu können, gezeichnet ist. Relativismus problematisiert den Gültigkeitsanspruch wissenschaftlicher Theorien und thematisiert wissenschaftliche Erkenntnis als menschliche Konstruktionsleistungen (Trautwein \& Lüdtke, 2004).

Die genannten Studien beschäftigten sich mit den Überzeugungen von Schülerinnen und Schülern sowie von Studierenden. Epistemologische Überzeugungen von Lehrkräften hingegen wurden vernachlässigt. Bereits vor mehr als zwanzig Jahren konnte die Forderung nach mehr Aufmerksamkeit für epistemologische Überzeugungen von Lehrkräften vernommen werden (Lampert, 1990). Ein Jahrzehnt später spricht Hofer (2001) von Lehrerüberzeugungen immer noch als von einem für die Lehrerforschung und Lehrerausbildung wichtigen Faktor, der noch immer nicht genügend Beachtung findet. Und 
auch jüngere Studien beklagen das geringe Interesse an epistemologischen Überzeugungen von Lehrkräften (Maggioni \& Parkinson, 2008; Olafson \& Schraw, 2006; Schraw \& Olafson, 2008). Die beiden Forschungsrichtungen, einerseits zu Epistemologien von Schülerinnen und Schülern sowie von Studierenden und andererseits von Lehrkräften verliefen parallel zueinander. Es gab nur wenige Versuche, die beiden Forschungslinien miteinander zu verbinden (Buehl \& Alexander, 2001; Schraw \& Olafson, 2002; Woolfolk-Hoy, Davis \& Pape, 2006). Der Grund hierfür könnte nach Meinung einiger Autoren darin bestehen, dass Lehrkräfte eine holistische Auffassung vom Wissen besitzen (Lehrer, 1990; Pollock, 1986). Während epistemologische Überzeugungen von Studierenden meist mithilfe der oben beschriebenen vier oder fünf separaten Dimensionen operationalisiert wurden (Hofer, 2000; Schommer-Aikins, 2002), lassen sich die holistischen epistemologischen Ansichten der Lehrkräfte mittels epistemologischer Weltbilder (engl. epistemological worldview) erfassen (Schraw \& Olafson, 2008). Basierend auf vorherigen Arbeiten (Cunningham \& Fitzgerald, 1996; Fielstein \& Phelps, 2001; Kincheloe, Slattery \& Steinberg, 2000; Kuhn, Cheney \& Weinstock, 2000) entwickelten Olafson und Schraw (2006) Vignetten zur Erfassung epistemologischer Weltbilder. Mit Hilfe der Vignetten werden drei verschiedene Weltsichten unterschieden: realistische Weltsicht, relativistische Weltsicht und kontextualistische Weltsicht, wobei die relativistische $(r=-.23)$ und kontextualistische Weltsicht $(r=-.37)$ negativ mit der realistischen Weltsicht zusammenhängen. Die hier berichteten Korrelationen wurden als Trends in den Daten interpretiert, da die Korrelationskoeffizienten aufgrund der kleinen Stichprobe $(N=17)$ nicht signifikant blieben. Lehrkräfte mit realistischen Ansichten glauben, dass sich das Wissen aus fest definierten, fertigen Bestandteilen zusammensetzt. Relativisten sind der Auffassung, dass jeder sein eigener Wissenskonstrukteur ist und dass sich das Wissen ständig verändert; und Kontextualisten denken, dass Schülerinnen und Schüler lernen, indem sie mit Lehrerunterstützung ein gemeinsames Verständnis vom Wissen konzipieren (Olafson \& Schraw, 2006). In der Studie von Olafson und Schraw (2006) wurden Lehrkräfte gebeten, sich einer, in der Vignette vorgestellten epistemologischen Weltsicht zuzuordnen (Schraw \& Olafson, 2002). Ein ähnliches Vorgehen wurde in einer Reihe von Interviewstudien praktiziert, indem Lehrkräfte einer bestimmten epistemologischen Sicht zugeordnet wurden (Levitt, 2001; White, 2000; Wilcox-Herzog, 2002).

Ein Forschungsbereich zu epistemologischen Überzeugungen bezieht sich auf das Fach Mathematik (McLeod, 1992; Schoenfeld, 1983; 1985). Die mit ausschließlich Mathematiklehrkräften durchgeführten Studien (Dubberke et al., 2008; Lipowsky, Thußbas, Klieme, Reusser \& Pauli, 2003; Peterson et al., 1989; Staub \& Stern, 2002) gehen in Bezug 
auf die verwendeten Instrumente auf Grigutsch, Raatz und Törner (1996) zurück. Dieser befasste sich mit der Philosophie des Faches Mathematik und unterscheidet zwischen vier Aspekten:

1) Der Anwendungsaspekt stellt Mathematik als Werkzeug zur Lösung praktischer Probleme dar.

2) Der Schemaaspekt betrachtet Mathematik als Sammlung von Verfahren und Regeln.

3) Der Prozessaspekt betont das kreative, explorative und entdeckende Moment der Mathematik.

4) Der Formalismusaspekt definiert Mathematik als durch Klarheit, Objektivität und Eindeutigkeit gekennzeichnet (vgl. Lipowsky et al., 2003).

Stipek et al. (2001) unterscheiden traditionelle und fragenentwicklende (engl. inquiryoriented) Lehrerüberzeugungen zur Mathematik. Dabei entspricht die traditionelle Vorstellung von Mathematik dem Schemaaspekt nach Grigutsch et al. (1996) und die fragenentwicklende dem Prozessaspekt (vgl. Stipek et al., 2001).

In Anlehnung an McComas und Olson (1998) unterscheiden Tsai (2006) und auch Seidel et al. (2008) konstruktivistische und empiristische epistemologische Überzeugungen. Eine ähnliche Unterscheidung findet sich ebenso bei Hashweh (1985, 1996). Bei einem konstruktivistischen Wissensverständnis wird die Vorläufigkeit von Wissen, die theoretisch begründete Qualität der wissenschaftlichen Exploration und die Rolle des Konzeptwechsels in der fortschreitenden Entwicklung wissenschaftlichen Verständnisses betont (Seidel et al., 2008). Kennzeichnend ist zudem die Annahme, dass Wissenschaft auf sozialen Aushandlungsprozessen und Vereinbarungen beruht, die zum Beispiel Paradigmen und akzeptierte Beweisführungen betreffen. Charakteristisch für eine empiristische Überzeugung ist demgegenüber das Verständnis, dass Wissenschaft eine objektive Realität aufdeckt, die unabhängig von individuellen Sichtweisen existiert (Tsai, 2006). Bei Schoenfeld (1985) finden sich Überzeugungen von Studierenden über das Wesen der Mathematik, über mathematisches Lernen und Problemlösen, welche - wie bereits beschrieben - in den Arbeiten von Schommer Anwendung fanden (1990, 1993, 1998).

Es lässt sich zusammenfassen, dass verschiedene Modelle epistemologischer Überzeugungen existieren und dementsprechend die empirische Forschung oft unterschiedliche Operationalisierungen von epistemologischen Überzeugungen vornimmt. So sind die Ergebnisse dieser Forschung vor dem Hintergrund verschiedener Forschungstraditionen zu verstehen. 


\subsection{3 Überblick über die Forschung zu epistemologischen Überzeugungen}

Insgesamt lassen sich unterschiedliche Bereiche in der Forschung zu epistemologischen Überzeugungen ausmachen: Es werden einerseits die Entwicklung bzw. die Veränderung epistemologischer Überzeugungen (Tsai, 2006) und andererseits deren Einfluss auf das Schülerlernen und unterrichtliches Handeln der Lehrkräfte (Seidel et al., 2008) untersucht. Zudem wird die Frage nach der Fachspezifität epistemologischer Überzeugungen diskutiert. In der aktuellen Forschung scheint sich der Konsens durchzusetzen, dass Menschen sowohl domänen-unabhängige, also allgemeine (Belenky, Clinchy, Goldberger \& Tarule, 1986; Buehl \& Alexander, 2001; Perry, 1970), als auch domänen-spezifische epistemologische Überzeugungen bzw. Überzeugungen über das akademische Wissen besitzen (Bromme, Kienhues \& Stahl, 2008; Buehl, Alexander \& Murphy, 2002; Muis, Bendixen \& Haerle, 2006). Dabei sollen allgemeine epistemologische Überzeugungen stabiler als domänen-spezifische sein (Gill, Ashton \& Algina, 2004) und im Laufe der Ausbildung immer mehr an Bedeutung verlieren, sodass domänen-spezifische Überzeugungen einflussreicher werden (Muis et al., 2006).

Im Gegensatz zum deutschsprachigen Raum stehen epistemologische Überzeugungen im angloamerikanischen Raum seit geraumer Zeit im Fokus der Forschung. Eine Reihe von Themenheften, erschienen in einschlägigen Fachzeitschriften (Kitchener, 2002; Sinatra, 2001), Überblicksartikel (Hofer \& Pintrich, 1997; Kuhn, 1999; Pillow, 1999) und ein Sammelband dokumentieren dieses Interesse (Hofer \& Pintrich, 2002). In Deutschland wird den Überzeugungen über Wissen, über seine Struktur und seine Genese erst in den letzten Jahren verstärkt Aufmerksamkeit geschenkt. Jedoch wurden bereits in den 1970er Jahren von der Kultusministerkonferenz (KMK) Lernziele wie das „Erkennen von Grenzen wissenschaftlicher Aussagen“ für die gymnasiale Oberstufe (KMK, 1977/1978, S. 561) oder das „Vermögen zur wissenschaftlichen Reflexion“ (Schmidt, 1991, S. 200) formuliert. Spätestens seitdem epistemologische Überzeugungen Eingang in bundesdeutsche Schulvergleichsstudien (z.B. TIMSS III: Baumert et al. 2000; TOSCA: Trautwein \& Lüdtke, 2004) gefunden haben, ist das Thema nicht mehr aus der empirischen Lehr-Lern-Forschung wegzudenken.

Im Kontext schulischen Lernens sind besonders die epistemologischen Überzeugungen der Schülerinnen und Schüler interessant, da diese das Lernen der Schülerinnen und Schüler beeinflussen. Die empirische Forschung demonstrierte bereits Zusammenhänge zwischen den epistemologischen Überzeugungen der Schülerinnen und 
Schüler (und auch Studierenden) und ihrem Lernerfolg (Hofer, 2000; Hofer \& Pinrich, 1997; Köller et al., 2000; Trautwein \& Lüdtke, 2004, 2007). Bereits 1984 konnte Ryan zeigen, dass Dualisten eher „Wissen“ und Relativisten verstärkt „Verständnis“ beim Lernen anstrebten, wobei das verständnisorientierte Lernen zu besseren Studienleistungen führte. Schommer (1990, 1993) konnte den Zusammenhang zwischen den von ihr aufgestellten Dimensionen epistemologischer Überzeugungen und der Schulnote sowie den Lernstrategien der Schülerinnen und Schüler zeigen, was in neueren Studien mehrmals repliziert wurde (Cano, 2005; Dahl, Bals \& Turi, 2005; Law, Chan \& Sachs, 2008; Zhang \& Watkins, 2001). Auch in der Studie von Hofer (2000) wurden signifikante Korrelationen zwischen den Studienleistungen und den Subskalen Sicherheit/Einfachheit des Wissens gefunden.

Die Rolle epistemologischer Überzeugungen wurde für eine Reihe für das Lernen relevanter Konstrukte demonstriert. Epistemologische Überzeugungen Studierender sollen zum Beispiel ihre Ziele und ihr Lernengagement sowie die Tiefe der Verarbeitungsprozesse und Kontrollmechanismen beim Lernen beeinflussen (Hofer \& Pintrich, 1997). Somit sind sie für das selbstregulierte Lernen von Bedeutung (Davis, 1997; Garrett-Ingram, 1997). Die Studie von Tsai (1998) machte deutlich, dass Schülerinnen und Schüler, die das Wissen als bereits vollständig entdeckt und fest bestimmt verstehen, verstärkt die Methode des Auswendiglernens anwendeten. Epistemologische Überzeugungen haben weiterhin einen Einfluss auf das Textverständnis (Schommer, 1994a). So ergaben sich bei Texten mit statistischen Inhalten negative Korrelationen zwischen Textverstehensleistung und einfachem Wissen (Schommer et al., 1992; Schommer, 1994a). Des Weiteren scheinen epistemologische Überzeugungen kognitive Verarbeitungsprozesse (Kardash \& Howell, 2000; Kardash \& Scholes, 1996) sowie die Konzeptentwicklung (Quian \& Alvermann, 1995) zu beeinflussen.

Wie bereits erwähnt, wurde den epistemologischen Überzeugungen von Lehrkräften bislang weniger Beachtung gewidmet als denen von Schülerinnen und Schülern (Hofer, 2001; Lampert, 1990; Maggioni \& Parkinson, 2008; Olafson \& Schraw, 2006; Schraw \& Olafson, 2008). Lehrer-Epistemologien sind jedoch in schulischen Lehr-Lern-Kontexten wichtig, auch wenn ihnen eine andere Bedeutung zukommt als Schüler-Epistemologien. Während es bei den Schülerinnen und Schülern um den Einfluss epistemologischer Überzeugungen auf das eigene Lernverhalten und die eigene Leistung geht, interessiert bei den Lehrer-Epistemologien deren Einfluss auf das Verhalten und die Leistung anderer - nämlich ihrer Schülerinnen und Schüler.

Die Forschung $\mathrm{zu}$ epistemologischen Überzeugungen ist im mathematischen und naturwissenschaftlichen Bereich stärker vertreten (Bell \& Linn, 2002; Elder, 2002; Hammer 
\& Elby, 2003; Op’t Exnde, De Corte \& Verschaffel, 2006; Stathopoulou \& Vosniadou, 2007). Es wurden bereits empirische Hinweise darauf geliefert, dass Lehrer-Epistemologien das unterrichtliche Handeln der Lehrkräfte beeinflussen (Etchberger \& Shaw '1992; Martens, 1992). Benson (1989) zeigte zum Beispiel, dass Lehrerüberzeugungen einen Einfluss auf das Curriculum ausübten, obwohl situationelle Faktoren das Unterrichtscurriculum nicht weniger bestimmen müssten. Bereits 1985 zeigte Hashweh, dass Lehrkräfte mit konstruktivistischen epistemologischen Überzeugungen ein breiteres Repertoire an Unterrichtsstrategien aufwiesen als ihre empirisch-orientierten Kolleginnen und Kollegen. Dabei wurden vier Arten von Unterrichtsstrategien unterschieden:

1) Lehrererklärung und Wiederholen als die am wenigsten effektive Unterrichtsmethode,

2) Präsentation verschiedener Beispiele, Analogien, Einsatz unterschiedlicher Demonstrationsmethoden und Schüleraktivitäten als eine etwas effektivere Methode,

3) Konfrontation der Schülerinnen und Schüler mit ihren Fehlkonzepten und Aufzeigen von Diskrepanzen zwischen diesen und den wissenschaftlichen Konzepten als eine deutlich effektivere Unterrichtsmethode sowie

4) Erläuterungen, die den Lernenden helfen, ihre Fehlkonzepte anzupassen, als die effektivste Methode (Hashweh, 1996).

Konstruktivistisch-orientierte Lehrkräfte verwendeten die effektiveren Unterrichtsstrategien als ihre empirisch-orientierten Kolleginnen und Kollegen. In der Folgestudie von Hashweh (1996) wurden ebenfalls die im Unterricht eingesetzten Methoden dieses Mal mittels Lehrerauskunft -sowie Lehrerbewertung von fehlerhaften Antworten der Schülerinnen und Schüler analysiert. Die Ergebnisse demonstrierten, dass die Anzahl der verwendeten Unterrichtsstrategien bei Lehrkräften mit konstruktivistischen epistemologischen Überzeugungen höher war als die bei Lehrkräften mit empiristischen epistemologischen Überzeugungen. Prozentuelle Angaben der verwendeten Unterrichtsstrategien demonstrierten, dass Lehrkräfte mit konstruktivistischen Überzeugungen besser in der Lage waren, Fehlkonzepte in den Schülerantworten zu identifizieren und die Rückmeldungen auf diese Fehlkonzepte zu beziehen. Sie konfrontierten ihre Schülerinnen und Schüler mit Gegenbeispielen, die die Schülerinnen und Schüler zum Überdenken ihrer Fehlkonzepte bewegen sollten. Hingegen gaben Lehrkräfte mit empiristischen Überzeugungen für fehlerhafte Antworten die Rückmeldung, dass die Antwort falsch ist und lieferten eine Erklärung der richtigen Antwort (Hashweh, 1996). 
Zusammenfassend kann festgehalten werden, dass die Bedeutung epistemologischer Überzeugungen von Lehrkräften für ihr unterrichtliches Handeln demonstriert worden ist (Hashweh, 1996, Tsai, 2006). Allerdings sind die epistemologischen Überzeugungen von Lehrkräften selbst nur unzureichend untersucht worden, da sich die bisherige Forschung auf epistemologische Überzeugungen der Schülerinnen und Schüler konzentrierte. Somit sind die meisten Messinstrumente zur Erfassung epistemologischer Überzeugungen in den Untersuchungen mit Schülerinnen und Schülern sowie Studenten entstanden.

\subsubsection{Messinstrumente zur Erfassung epistemologischer Überzeugungen}

Die Forschung zu epistemologischen Überzeugungen im angloamerikanischen Raum weist eine lange Tradition auf und die meisten Erhebungsinstrumente sind in Anlehnung an englischsprachige Instrumente und insbesondere an den „Schommer Epistemological Questionnaire“ (SEQ) (Schommer, 1990, 1993) entstanden. Dieser Fragebogen wurde in Original- und in adaptierter Form in einer Mehrzahl von Studien eingesetzt (z.B. Braten \& Stromso, 2005; Hall, Chiarello \& Edmonson, 1996; Hofer, 2000; Jehng, Johnson \& Anderson, 1993; Kardash \& Scholes, 1996; Pahn, 2008; Rukavina \& Daneman, 1996; Schommer, 1990, 1993).

Schommer (1990) postulierte fünf Dimensionen epistemologischer Überzeugungen: a) Struktur, b) Sicherheit, c) Quelle des Wissens, d) Lernfähigkeit und e) Lerngeschwindigkeit (Schommer, 1998, S. 47). Zur Messung der Dimensionen wurden 63 Items zu den jeweiligen Ansichten bezüglich des Wissens und des Lernens erstellt, die zwölf inhaltliche Bereiche ansprechen: zum Beispiel, dass Lernen entweder direkt erfolgt, sodass jegliche Kraftinvestition in Lernen eine verlorene Zeit darstellt, dass das Wissen sicher und Lernerfolg unabhängig vom „harten Arbeiten“ ist oder dass die Lernfähigkeit angeboren ist (Schommer, Crouse \& Rhodes, 1992). Tabelle 1 präsentiert Beispielitems aus jedem Bereich. Hinsichtlich naiver Überzeugungen haben 28 Items eine negative Valenz (Beispielitem: „You never know what a book means unless you know the intent of the author") und 35 Items eine positive Valenz (Beispielitem: „It's a waste of time to work on problems which have no possibility of coming out with a clear-cut and unambiguous answer") (Schommer et al., 1992, S. 437). Hohe Werte auf der Gesamtskala bedeuten somit eine hohe Präsenz naiver Überzeugungen. 
Tabelle 1

Beispielitem für die Itemgruppen aus dem SEQ (1990)

Gruppe Beispielitem

Learning is quick

Can't learn how to learn

Learn first time

Concentrated effort is a waste time

Success is unrelated to hard work

Avoid ambiguity

Seek single answers

Avoid integration

Depend in authority

Ability to learn is innate

Don't criticize authority

Knowledge is certain
Successful students learn things quickly.

Self help books are not much help.

Almost all the information you can learn from a textbook you will get during the first reading.

If a person tried too hard to understand a problem, they will most likely just end up being confused.

The really smart students don't have to work hard to do well in school.

I don't like movies that don't have an ending.

Most words have one clear meaning.

When I study I look for specific facts.

How much a person gets out of school depends on the quality of instruction.

An expert is someone who has a special gift in some area.

People who challenge authority are overconfident.

Scientists can ultimately get to the truth.

In Studien mit Studierenden (Schommer, 1992), Schülerinnen und Schüler der Sekundarstufe (engl. high school students) (Schommer, 1993) und Erwachsenen (Schommer, 1998) konnten vier der fünf angenommenen Dimensionen bestätigt werden: einfaches Wissen und sicheres Wissen sowie Lernfähigkeit und -geschwindigkeit (Schommer, 1990; Schommer, Clavert, Ganglietti \& Baja, 1997; Schommer et al., 1992). Allerdings wurde oft die Faktorstruktur der entwickelten Items kritisiert (Hofer \& Pintrich, 1997). In der Studie von Quian und Alvermann (1995) mit 212 Schülerinnen und Schülern der Sekundarstufe luden nur 32 von 63 Items des SEQ auf drei Faktoren: Lernfähigkeit, schnelles Lernen sowie einfaches-sicheres Wissen. Diese stellten bei Schommer noch zwei getrennte Faktoren dar. 
Der Fragebogen „Discipline-Focused Epistemological Beliefs Questionnaire“ (DEBQ) wurde zur bereichsspezifischen Erfassung epistemologischer Überzeugungen entwickelt: Beispielitem „In this field knowledge is certain“ (Hofer, 2000). Beim Vergleich der Fächer Psychologie und Naturwissenschaften (Biologie, Chemie, Physik) wurden für beide Disziplinen vier Faktoren identifiziert:

a) Einfachheit/Sicherheit des Wissens (Beispielitem: ,Most of what is truth in this subject is already known"),

b) Rechtfertigungszusammenhang (Beispielitem: „First-hand experience is the best way of knowing something in this field"),

c) Quelle des Wissens (Beispielitem: „If you read something in a textbook for this subject, you can be sure that it is true") und

d) Erreichbarkeit der Wahrheit (engl. attainability of truth) (Beispielitem: „Experts in this field can ultimately get to the truth").

Die beiden von Schommer (1990, 1992, 1993) postulierten Faktoren sicheres und einfaches Wissen ließen sich empirisch jedoch nicht trennen.

Es existieren eine Reihe weiterer englischsprachiger Instrumente, zum Beispiel „Reflective Judgment Interview“ nach King und Kitchener (1994), „Beliefs About Knowledge and Learning“ nach Jehng et al. (1993), „Epistemologic Belief Inventory“ (EBI) nach Schraw, Dunkle und Bendixen (1995). Im deutschsprachigen Raum können folgende Instrumente erwähnt werden: der neu entwickelte „Fragebogen zur Erfassung des Entwicklungsniveaus epistemologischer Überzeugungen“ (FREE) von Krettenauer (2005), bei dem die Probanden zu 14 kontroversen Themen aus Natur- und Geisteswissenschaften Stellung nehmen müssen. Der Fragebogen „Connotative Aspects of Epistemological Beliefs“ (CAEB) von Stahl und Bromme (2007) verwendet Adjektivpaare wie „dynamisch - statisch“ oder „objektiv subjektiv“, um semantisch unterschiedliche Konnotationen einer Disziplin zu messen. Im Rahmen der Studie „Transformation des Sekundärschulsystems und akademische Karrieren“ (TOSCA) (z.B. Köller, Watermann, Trautwein \& Lüdtke, 2004) wurden domänenübergreifende epistemologische Überzeugungen der Schülerinnen und Schüler mit einer relativ kurzen Skala erhoben (Trautwein \& Lüdtke, 2004). Grundlage für die insgesamt 15 Items, die sich auf die Kerndimension „Struktur des Wissens“ beziehen, waren die Skalen von Hofer (2000) und Schommer (1993) in einer Adaption durch Schiefele, Moschner und Husstegge (2002). Der Bogen umfasst eine Subskala zu Dualismus und eine zu Relativismus. Die Dualismusskala (5 Items) thematisiert das Vertrauen in wissenschaftliche Erkenntnisse sowie in die Fähigkeit der Wissenschaft, eines Tages alles erklären zu können. Beispielitems 
sind „Die wissenschaftliche Forschung zeigt, dass es auf die meisten Probleme eine eindeutig richtige Antwort gibt“ oder etwa „Wissenschaftliche Erkenntnis ist über jeden Zweifel erhaben“. Hohe Werte auf dieser Skala signalisieren ein unkritisches Verhältnis zu wissenschaftlichen Erkenntnissen. Ein Beispielitem für die Relativismusskala lautet „Wissenschaftliche Theorien können sich auch jederzeit als falsch erweisen“. Eine hohe Ausprägung auf dieser Skala signalisiert, dass man kritisch mit wissenschaftlichen Theorien umgeht und dass einem bewusst ist, dass sie das Ergebnis menschlicher Konstruktionsleistungen sind. Da die beiden Subskalen eine moderate Interkorrelation $(r=-$ $.36, p<.01)$ aufweisen, sprechen die Autoren bei den Skalen nicht von Endpunkten einer gemeinsamen Dimension, sondern von zwei Konstrukten. Dieser Fragebogen kann als ein valides Instrument angesehen werden und verspricht aufgrund seiner relativ kurzen Skalen eine ökonomische Datenerhebung. Daher soll dieser Fragebogen in der vorliegenden Arbeit im Rahmen der dritten Studie verwendet werden, um das neu entwickelte Instrument zur Messung von Überzeugungen zum Lehren und Lernen zu validieren.

Die meisten deutschsprachigen Instrumente orientieren sich an dem SEQ (Schommer, 1993) sowie an der überarbeiteten Übersetzung von Gruber und Renkl (1995) (Salhab, 2003). Der Fragebogen stellt eine auf 28 Items reduzierte Version des Instruments dar. Die Items sind auf einer fünfstufigen Skala $(1=$ trifft nicht $\mathrm{zu}$, bis $5=$ trifft $\mathrm{zu}) \mathrm{zu}$ beantworten, wobei eine hohe Zustimmung naive Überzeugungen und eine niedrige Zustimmung entwickelte Überzeugungen einer Person wiedergibt. Der Fragebogen umfasst fünf Skalen: einfaches Wissen (Beispielitem: „Die meisten Wörter haben eine klare Bedeutung“), sicheres Wissen (Beispielitem: „Wissenschaftler können letztlich zur Wahrheit kommen“), allwissende Autorität (engl. omniscient authority) (Beispielitem: „Auch der Rat von Fachleuten ist oft anzuzweifeln“), angeborene Lernfähigkeit (Beispielitem: „Unterschiede in der Lernfähigkeit sind angeboren“) und schnelles Lernen (Beispielitem: „Erfolgreiche Studenten verstehen Lerninhalte schnell“"). In dieser Version sind 10 Items mit einer negativen Valenz (Beispielitem: „Es liegt am jeweiligen Studenten, wie viel er aus dem Lehrbuch lernt“) und 17 Items mit einer positiven Valenz (Beispielitem: „Wenn ich lerne, präge ich mir spezifische Fakten ein“) versehen. Dieses Instrument stellt die Möglichkeit dar, neben den allgemeinen Überzeugungen zur Struktur des Wissens auch epistemologische Überzeugungen bezüglich des Lernens (Lernfähigkeit und -geschwindigkeit) zu erheben. Im Zusammenhang mit den vier Lehrerüberzeugungen zum Lehren und Lernen ist von Interesse, welche dieser epistemologischen lernspezifischen Überzeugungen mit Transmission, Konstruktion, Partizipation und Schülerorientierung zusammenhängen. So soll dieses Instrument in der 
dritten Studie der vorliegenden Arbeit verwendet werden, um Zusammenhänge zwischen epistemologischen Überzeugungen und den vier Überzeugungen zum Lehren und Lernen, erhoben mit dem neu entwickelten Instrument, zu untersuchen.

Zusammenfassend lässt sich festhalten, dass unterschiedliche Instrumente zur Erfassung epistemologischer Überzeugungen vorliegen. Einige davon, so wie der SEQ (Schommer, 1990, 1993), haben sich, trotz Kritik, in einer Reihe empirischer Studien bewährt. In Orientierung an den SEQ wurden neue Instrumente entwickelt und an größeren Stichproben validiert. Beispielsweise können der Fragebogen aus der TOSCA-Studie (z.B. Trautwein \& Lüdtke, 2004) und die deutsche Version des SEQ (Gruber \& Renkl, 1995) als valide Instrumente gelten. Daher sollen im Rahmen der vorligeneden Arbeit diese zwei Instrumente verwendet werden, einerseits zur Validierung des neu entwickelten Instruments und andererseits zur Analyse der Beziehung zwischen diesen und den Überzeugungen zum Lehren und Lernen - Transmission, Konstruktion, Partizipation und Schülerorientierung. 


\subsection{Epistemologische Überzeugungen und lehr-/lernbezogene Überzeugungen}

In der Lehrerforschung werden lehr-/lernbezogene Überzeugungen von Lehrkräften und auch deren epistemologische Überzeugungen seit längerer Zeit als für die Unterrichtspraxis und für die Schülerleistung wichtige Faktoren diskutiert (Hollingsworth, 1989; Nespor, 1987; Pajares, 1992) und empirisch untersucht (Dubberke et al., 2008; Hartinger et al., 2006; Seidel et al., 2006). Allerdings fehlt bis heute eine einheitliche Definition der Begriffe. Dies lässt sich in den Operationalisierungen beider Konstrukte erkennen, vor allem hinsichtlich der Frage, in welcher Relation epistemologische Überzeugungen zu lehr-/lernbezogenen Überzeugungen stehen, welche zum Teil große Diskrepanzen aufweisen (Dubberke et al., 2008; Chan \& Elliott, 2004; Seidel et al., 2008). Einige Studien fassen epistemologische Überzeugungen als einen Teilbereich der Überzeugungen zum Lehren und Lernen auf (Aguirre \& Speer, 2000; Dubberke et al., 2008), andere klammern Wissensansichten bei Untersuchungen von Lehr-Lern-Überzeugungen gänzlich aus (Cheng, 2009; Kember, 1997; Staub \& Stern, 2002). Explizit beschäftigen sich nur sehr wenige Untersuchungen mit der Frage nach dem Zusammenhang der beiden Überzeugungen zueinander (Can \& Elliott, 2004; Cheng et al., 2009; Seidel et al., 2008).

Die Unterschiede in der Operationalisierung der Überzeugungen lassen sich auf verschiedene Traditionen zurückführen. Eine Tradition stammt aus der Kritik der Forschung zu Lehrerüberzeugungen ohne Fachbezug (Peterson et al., 1989). Diese Kritik basierte auf der Annahme, dass das Expertenwissen in höchstem Maße als bereichsspezifisch zu betrachten ist (Chi, Glaser \& Farr, 1988; Sternberg \& Horvath, 1999). Auch vor dem Hintergrund der Arbeiten Shulmans (Shulman, 1986, 1987), die auf den Mangel früherer Untersuchungen hinweisen, Lehrerwissen und Lehrerüberzeugungen unabhängig vom Fachbezug zu untersuchen, richtete sich die Aufmerksamkeit der Forschung auf die Struktur und Funktion fachspezifisch-pädagogischen Wissens. So wurde, wie bereits erwähnt, verstärkt die Forschung zu Lehrerüberzeugungen im Fach Mathematik betrieben (Aguirre \& Speer, 2000; Clausen, Reusser \& Klieme, 2003; Hashweh, 1985, 1996; Lipowsky et al., 2003; Stipek et al., 2001). In Mathematik werden unter Lehrerüberzeugungen sowohl Überzeugungen über das Wesen von Mathematik als auch Überzeugungen über das Lehren und Lernen verstanden (Ernest, 1989; Thompson, 1984, 1985, 1992). In der deutschsprachigen Forschung lässt sich die Tendenz, epistemologische Überzeugungen als Teilbereich der Lehr-Lern-Überzeugungen zu betrachten, ebenfalls erkennen (Dubberke et al., 2008; Lipowsky et al., 2003). So wurden in der Teilstudie des COACTIV-Projekts von Dubberke et al. (2008) lerntheoretische 
Überzeugungen der Lehrkräfte mit einer Skala zu epistemologischen Überzeugungen nach Grigutsch et al. (1996) - „Mathematik als Toolbox“ - sowie Skalen nach Fennema et al. (1990) zu Mathematiklernen - „Rezeptives Lernen durch Beispiele und Vormachen“, „Eindeutigkeit des Lösungsweges“ und „Einschleifen von technischem Wissen“ - erhoben. Auch in der Vergleichsstudie deutscher und schweizerischer Mathematiklehrkräfte von Lipowsky et al. (2003) wurden beide Konstrukte für die Analyse von Lehrerüberzeugungen im Fach Mathematik untersucht: die Philosophie des Schulfaches (Skala nach Grigutsch et al., 1996) und Skalen zum fachspezifischen pädagogischen Wissen nach Fennema et al. (1990) in der deutschen Adaption von Staub und Stern (2002).

Dagegen wurden Lehr-Lern-Überzeugungen von Lehrkräften aus dem universitären Bereich ohne Beachtung epistemologischer Einsichten untersucht. Es wurden mehrere Überzeugungen (z.B. Gow \& Kember, 1993; Kember 1997; Norton et al., 2005; Pratt et al., 2001; Trigwell et al., 1994) von Vermittlung von Wissen bis hin zur Förderung von Lernprozessen (Kember, 1997) sowie lehrer-orientierten vs. schüler-orientierten Lehrkonzeptionen unterschieden (Trigwell \& Prosser, 2004). Diese Forschungsrichtung blieb bereichsunspezifisch und verglich dann die Überzeugungen von Lehrkräften verschiedener Fächer (Norton et al., 2005; Pratt et al., 2001). Es kann vermutet werden, dass gerade in der bereichsunspezifischen Forschung zu Lehrerüberzeugungen aus dem universitären Bereich epistemologische Überzeugungen nicht als Teil der Lehr-Lern-Überzeugungen angesehen werden. Allerdings wurden auch im Schulkontext in den Studien mit Mathematiklehrkräften, zum Beispiel bei Staub und Stern (2002) und bei Peterson et al. (1989), keine Überzeugungen über das Fach, sondern ausschließlich lehr-/lerntheoretische Überzeugungen erhoben: die Rolle des Lerners, die Relation zwischen Fähigkeiten, dem Verständnis und dem Problemlösen, der Sozialkonstruktivismus und die Rolle des Lehrers. Hartinger et al. (2006) beschäftigen sich ähnlich - ohne epistemologische Überzeugungen mitzuerheben - mit allgemeinen, also bereichsunspezifischen Lehr-Lern-Vorstellungen von Grundschullehrkräften in Anlehnung an Morine-Dershimer und Kent (1999), die die Frage nach der Bedeutung der eher allgemeinen und invariant über den fachlichen Kontexten stehenden Vorstellungen zum Lehren und Lernen für das unterrichtliche Handeln aufwarfen.

Somit soll im Rahmen der vorliegenden Arbeit eine Trennung zwischen den beiden Konstrukten eingehalten werden. Obwohl beide Konstrukte Lehrerüberzeugungen beschreiben, befinden sie sich konzeptuell auf unterschiedlichen Ebenen - mit Pajares Worten (1992) liegen die einen eher im Zentrum und die anderen verstärkt an der Peripherie der Überzeugungssysteme. Epistemologische Überzeugungen sind dafür verantwortlich, dass eine 
Lehrkraft an die Begabung eines Schülers glaubt oder aber daran, dass ein Schüler einen Sachverhalt „nie lernt, wenn er [ihn] nicht beim ersten Mal verstanden hat“ (ein Item aus dem SEQ nach Schommer, 1900, 1993); dagegen lassen Überzeugungen zum Lehren und Lernen eine Lehrkraft den Vorgang des Lernens beispielsweise als eine eigene Wissenskonstruktionsleistung auffassen.

Gleichwohl wurde die Verbindung zwischen epistemologischen Überzeugungen und Überzeugungen zum Lehren und Lernen in der Literatur oft thematisiert (Clark \& Peterson, 1986; Fennema \& Loef Franke, 1992; Marland, 1995; 1998; Richardson, 1996). Olafson und Schraw (2006) gingen in zwei Studien mit Grundschullehrern $(n=22 ; n=18)$ unter anderem der Frage nach, in welcher Beziehung epistemologische Weltansichten zu epistemologischen Überzeugungen stehen. Zusätzlich zu den Vignetten zu epistemologischen Weltansichten wurden fünf Skalen zur Messung epistemologischer Überzeugungen (Epistemological Belief Inventory, Schraw, Bendixon \& Dunkle, 2002) eingesetzt: Struktur des Wissens, Sicherheit des Wissens, Quelle des Wissens, Lernfähigkeit und Lerngeschwindigkeit. Die Ergebnisse zeigten, dass alle fünf Skalen positiv mit der realistischen Weltsicht korreliert waren. So glaubten Lehrkräfte mit der realistischen epistemologischen Weltsicht an die angeborenen Lernfähigkeiten $(r=.56)$, an die Einfachheit des Wissens $(r=.37)$ und an das schnelle Lernen $(r=.30)$. Wegen der kleinen Stichprobengröße $(n=22 ; n=18)$ wurde von diesen Angaben als statistisch nicht signifikant und lediglich als Trend in den Daten berichtet (Olafson \& Schraw, 2006). Dagegen waren Lehrkräfte mit kontextualistischer Weltsicht einer anderen Auffassung: Die Korrelationen zwischen Kontextualismus-Werten und epistemologischen Überzeugungen fielen alle negativ aus. Diese Lehrkräfte glaubten eher nicht daran, dass die Fähigkeit zum Lernen angeboren ist $(r=-.41)$ und dass das Lernen entweder schnell oder gar nicht möglich ist $(r=-.37)$. Dieses Ergebnis bestätigte das Ergebnis der ersten Teilstudie, nämlich dass eine realistische Weltansicht mit naiven epistemologischen Überzeugungen und eine kontextualistische und relativistische Weltansicht mit fortgeschrittenen epistemologischen Überzeugungen zusammenhängen. Auf dieser Grundlage formulierten Olafson und Schraw (2006) die Annahme, dass eine bestimmte epistemologische Vorstellung eine bestimmte Vorstellung vom Lernen mit sich bringt. So sollen Lehrkräfte, die das Wissen als einfach und gesichert auffassen, eine rezeptive Überzeugung zum Lernen besitzen, und Lehrkräfte, die das Wissen als ein Netzwerk von Theorien verstehen, der Überzeugung sein, dass Schülerinnen und Schüler durch Verständigungs- und Aushandlungs-Prozesse ihr Wissen selbst konstruieren. 
Seidel et al. (2008) untersuchten in ihrer Studie mit 50 deutschen Physiklehrkräften lehr-/lerntheoretische Überzeugungen (Fennema et al., 1990) und epistemologische Überzeugungen (Köller et al., 2000) sowie deren Beziehung zueinander. Es wurde vermutet, dass das empiristische Wissensverständnis der Lehrkräfte mit deren rezeptiver Überzeugung zum Lernen zusammenhängt. Dagegen sollen Lehrkräfte, die Wissen als ein Netzwerk von Theorien verstehen, welches sich ständig durch neue Erkenntnisse verändert (konstruktivistisches Wissensverständnis), ihre Aufgabe in der Bereitstellung von Lernumgebungen sehen, welche Lernende in den Prozess des Wissensaufbaus und der Generierung von Wissen einbinden. Die Ergebnisse der Studie von Seidel et al. (2008) bestätigen diese Annahme: Es wurde ein positiver signifikanter Zusammenhang des konstruktivistischen Wissensverständnisses mit der konstruktivistischen Überzeugung zum Schülerlernen: $r=.41, p<.005$ sowie ein positiver signifikanter Zusammenhang eines empiristischen Wissensverständnisses mit einer rezeptiven Überzeugung zum Schülerlernen: $r$ $=.28, p<.05$ festgestellt.

Chan und Elliott (2004) untersuchten ebenfalls die Beziehung von Überzeugungen zum Lehren und Lernen und epistemologischen Überzeugungen. In Orientierung an Dunkin und Precians (1992) sprechen sie bei ersteren von Lehr-Lern-Konzeptionen und unterscheiden a) eine traditionelle, die der Vorstellung vom Lehren als einem einfachen Transfer von Wissen vom Experten zum Novizen entspricht sowie Lernen als Aufnahme von diesem Wissen auffasst, und b) eine konstruktivistische Konzeption, die Lehren als Unterstützung vom Prozess der Wissenskonstruktion durch Argumentation und Diskussion definiert (Chan \& Elliott, 2004). Bei epistemologischen Überzeugungen verwendeten sie einen an das Instrument von Schommer (1990, 1994b) orientierten Fragebogen, der vier Faktoren umfasst: angeborene Lernfähigkeit, Lernanstrengung (engl. learning effort/process), Wissen der Experten und sicheres Wissen. Die Ergebnisse zeigen einen positiven Zusammenhang zwischen einer traditionellen Vorstellung von Lehren und Lernen und dem Glauben an eine angeborene Lernfähigkeit $(r=.40, p<.001)$, Wissen der Experten $(r=.40, p<.001)$ und sicherem Wissen $(r=.31, p<.001)$ sowie einen negativen Zusammenhang konstruktivistischer Vorstellung von Lehren und Lernen und angeborener Lernfähigkeit ( $r=$ $.16, p<.01)$ und Wissen des Experten $(r=-.22, p<.001)$, aber einen positiven Zusammenhang mit Lernanstrengung $(r=.39, p<.001)$. Zudem wurde die Annahme eines kausalen Zusammenhangs zwischen epistemologischen Überzeugungen und Überzeugungen zum Lehren und Lernen mithilfe von Strukturgleichungsmodellen überprüft. Das Ergebnis zeigte einen signifikanten Effekt vom Glauben an eine angeborene Lernfähigkeit $(\beta=.52, t=$ 
6.45, $p<.05)$, Wissen des Experten $(\beta=61, t=5.88, p<.05)$ und sicherem Wissen $(\beta=.46, t$ $=5.66, p<.05)$ auf die traditionelle Vorstellung von Lehren und Lernen sowie vom Glauben an Lernanstrengung $(\beta=-.56, t=-7.01, p<.05)$ auf die konstruktivistische Vorstellung von Lehren und Lernen.

In der Studie von Cheng et al. (2009) wurde erneut die Beziehung zwischen epistemologischen Überzeugungen und Konzeptionen zum Lehren und Lernen überprüft, dieses Mal mithilfe quantitativer und qualitativer Methoden. Im Einklang mit der Studie von Chan und Elliott (2004) wurde bei zwölf zukünftigen Lehrkräften mit fortgeschrittenen epistemologischen Vorstellungen eine konstruktivistische Vorstellung von Lehren und Lernen vorgefunden. Allerdings wurden auch bei vier Studierenden mit fortgeschrittenen epistemologischen Vorstellungen eine Kombination aus konstruktivistischen und traditionellen Vorstellungen von Lehren und Lernen identifiziert (Cheng et al., 2009, S. 324).

Man kann also festhalten, dass fortgeschrittene epistemologische Überzeugungen eher $\mathrm{zu}$ konstruktivistischen Lehr-Lern-Überzeugungen führen und naive epistemologische Überzeugungen zu traditionellen (rezeptiven) Lehr-Lern-Überzeugungen. Gleichwohl lieferte die empirische Forschung gegensätzliche Hinweise, und zwar, dass fortgeschrittene epistemologische Überzeugungen mit traditionellen Lehr-Lern-Vorstellungen zusammenhängen können (Cheng et al., 2009), sodass die Frage nach dem Zusammenhang zwischen epistemologischen und lehr-/lernbezogenen Überzeugungen als nicht eindeutig beantwortet gilt.

Zudem sind die Ergebnisse bisheriger Forschung wegen ihrer unterschiedlichen methodischen Zugänge sowie aufgrund der unterschiedlichen Operationalisierungen der Konstrukte nicht ohne weiteres vergleichbar. Zum Beispiel unterschieden Seidel et al. (2008) empiristische und konstruktivistische epistemologische Überzeugungen und traditionelle und konstruktivistische Lehr-Lern-Überzeugungen. Chan und Elliott (2004) sowie Cheng et al. (2009) hingegen untersuchten dagegen fünf Dimensionen epistemologischer Überzeugungen und deren Einfluss auf konstruktivistische bzw. traditionelle Lehr-Lern-Überzeugungen. Weitere Untersuchungen zum Zusammenhang zwischen epistemologischen und lehr/lernbezogenen Überzeugungen sind daher wünschenswert und notwendig.

Vor allem in Bezug auf die noch wenig untersuchten partizipatorischen und schülerorientierten Lehr-Lern-Überzeugungen besteht deutlicher Forschungsbedarf. Die bisherige Forschung konzentrierte sich auf zwei Lehr-Lern-Überzeugungen, die konstruktivistische und die transmissive Überzeugung. Der Zusammenhang zwischen epistemologischen und den lehr-/lernbezogenen Überzeugungen Partizipation oder 
Schülerorientierung blieb bisher ungeklärt. In der vorliegenden Arbeit soll versucht werden, dieses Defizit zu begleichen. 


\section{$3 \quad$ Ableitung der Fragestellungen}

Ausgehend vom aktuellen Stand der Forschung werden im folgenden Kapitel die Fragestellungen zu den empirischen Studien abgeleitet.

Das zentrale Anliegen der vorliegenden Arbeit besteht in der Untersuchung der vier Überzeugungen Transmission, Konstruktion, Partizipation und Schülerorientierung. Es wird als essenziell und somit für die Forschung von großem Nutzen erachtet, diese vier Überzeugungen mithilfe von qualitativen und quantitativen Methoden zu analysieren und messbar zu machen. Im Fokus der ersten Interviewstudie steht zunächst eine inhaltsanalytische Untersuchung der Überzeugungen Transmission, Konstruktion, Partizipation und Schülerorientierung, wobei vor allem die letzten beiden aufgrund mangelnder Forschung von besonderem Interesse sind. Weiterhin richtet sich die Aufmerksamkeit auf die Bedeutung dieser vier Überzeugungen in Abhängigkeit vom Zeitpunkt in der Lehrerausbildung und Lehrerlaufbahn. Dabei werden im Rahmen der ersten Studie die Überzeugungen von Studierenden zu drei Zeitpunkten ihrer Lehrerausbildung, von angehenden Lehrkräften (Referendaren) und solchen mit Berufserfahrung miteinander verglichen. Im Rahmen der zweiten Studie wird ein Fragebogen zur Erfassung der vier Überzeugungen - Transmission, Konstruktion, Partizipation und Schülerorientierung entwickelt. Die Reliabilität und Validität des neuen Instruments sollen in einer Studie mit Lehramtsstudierenden bestimmt werden. Zudem werden die Überzeugungen von Bachelorund Masterstudierenden im Querschnittsdesign miteinander verglichen, um der Frage nach der Bedeutung von Überzeugungen zu verschiedenen Zeitpunkten in der Lehrerausbildung nachzugehen. Im Rahmen der dritten Studie sollen ebenfalls Überzeugungen von Studierenden zu drei unterschiedlichen Zeitpunkten ihres Studiums verglichen werden. Jedoch steht im Zentrum der dritten Studie der Zusammenhang zwischen epistemologischen und den lehr-/lernbezogenen Überzeugungen Transmission, Konstruktion, Partizipation und Schülerorientierung. Im Folgenden werden die drei empirischen Untersuchungen und deren konkrete Forschungsfragen skizziert. 


\section{1 Überzeugungen zum Lehren und Lernen zu unterschiedlichen Zeitpunkten in der Lehrerlaufbahn (Studie 1)}

Aufgrund des starken Interesses der empirischen Forschung für das Überzeugungspaar Transmission und Konstruktion wurden andere Überzeugungen zum Lehren und Lernen bislang vernachlässigt. Studien, die sich explizit mit der Überzeugung Partizipation (Martínez et al., 2001) oder Schülerorientierung (Pratt, 2005) beschäftigen, finden sich nur vereinzelt. Jedoch ist die Bedeutung solcher Ansichten zum Lehren und Lernen unumstritten, worauf in bedeutenden Lernansätzen und Theorien, wie zum Beispiel im Lerngemeinschaftsansatz nach Lave \& Wenger (1991), im Konstrukt der Schülerorientierung nach Klieme et al. (2006) oder in der Humanismus-Theorie des Lernens nach Rogers (1969) hingewiesen wird. Außerdem wurde die Bedeutung dieser Überzeugungen besonders für angehende Lehrkräfte demonstriert (Brookhart \& Freeman, 1992; Hollingsworth, 1989; Wan Ng et al., 2010). Deren Ideen wurden, wenn auch nur implizit, in die Lehrerkompetenzmodelle aufgenommen: Wertbindungen mit der Fürsorge und dem Fördern und Fordern von Schülerleistung im COACTIV-Modell der Lehrerkompetenzen (Baumert \& Kunter, 2006) oder Berufsmoral nach Oser (1998).

In der Tat kann nicht davon ausgegangen werden, dass in den Überzeugungen von Lehrkräften nur die zwei Sichtweisen Transmission und Konstruktion existieren. Um der Komplexität der Lehrerüberzeugungen jedoch annähernd gerecht zu werden, sollen in der ersten Studie der vorliegenden Dissertation zusätzlich zu den Überzeugungen Transmission und Konstruktion auch partizipatorische Vorstellungen und Ideen der Schülerorientierung untersucht werden. Dabei empfiehlt sich für die erste Studie die qualitative Erhebungs- und Analysemethode zur detaillierten Erfassung der vier Überzeugungen. Es sollen Interviews sowohl mit zukünftigen als auch mit erfahrenen Lehrkräften zu ihren Ansichten über das Lehren und Lernen durchgeführt werden. Die Orientierung an entsprechenden theoretischen Lernansätzen wird dabei stets beibehalten.

Zugleich soll die erste Studie die Frage nach der Bedeutung dieser vier Überzeugungen zu unterschiedlichen Zeitpunkten in der Lehreraubildung und Lehrerlaufbahn verfolgen. Durch ein Querschnittsdesign soll der Vergleich von Überzeugungen von zukünftigen Lehrkräften in der ersten Phase der Lehrerausbildung - Studierende am Anfang, in der Mitte und am Ende ihres Studiums - mit Überzeugungen von Lehrkräften in der zweiten Phase der Lehrerausbildung - Referendare - sowie Überzeugungen von erfahrenen Lehrkräften durchgeführt werden. So können Rückschlüsse darauf gezogen werden, inwieweit 
die Lehrerausbildung und die Lehrerlaufbahn für die Entwicklung der Überzeugungen bedeutsam sind. Insgesamt ergeben sich für die erste Studie folgende Fragen:

1) Inwieweit lassen sich die in der einschlägigen Literatur identifizierten Überzeugungen Transmission, Konstruktion, Partizipation und Schülerorientierung in der Selbstauskunft der (angehenden) Lehrkräfte zu ihren Ansichten vom Lehren und Lernen identifizieren?

2) Inwieweit unterscheiden sich Lehrkräfte $\mathrm{zu}$ verschiedenen Zeitpunkten der Lehrerausbildung und Lehrerlaufbahn in ihren Überzeugungen zum Lehren und Lernen?

Es ist zu erwarten, dass alle vier Überzeugungen in den Lehreransichten identifiziert werden. Allerdings liegt die Vermutung nahe, dass diese Überzeugungen unterschiedlich starker Ausgeprägung unterliegen. Sowohl in der Gesamtheit der befragten Lehrkräfte als auch in den Aussagen der Lehrkräfte zu verschiedenen Zeitpunkten in der Lehrerausbildung und Lehrerlaufbahn werden Unterschiede erwartet. 


\subsection{Konstruktion des Fragebogens zur Messung der vier Lehrerüberzeugungen zum Lehren und Lernen (Studie 2)}

Auf der Grundlage der ersten Interviewstudie sowie in Anlehnung an theoretische Lernansätze und existierende Instrumente soll ein Instrument zur Erfassung von Überzeugungen zum Lehren und Lernen - Transmission, Konstruktion, Partizipation und Schülerorientierung - entwickelt werden. Dabei besteht das Ziel in der Analyse der vier lehr/lernbezogenen Überzeugungen, und zwar - im Gegensatz zur ersten Interviewstudie mit der qualitativen Inhaltsanalyse - mittels faktorenanalytischer Analysen des Fragebogens anhand einer Stichprobe von Lehramtsstudierenden. Die in der Faktorenanalyse erhaltenen Faktoren sollen auf ihre Übereinstimmung mit den theoretisch formulierten Dimensionen der vier Überzeugungen überprüft werden. Zur Überprüfung der Konstruktvalidität des Fragebogens werden weitere Instrumente eingesetzt, die inhaltsnahe Konstrukte erfassen. Zum einen soll der Fragebogen zur Messung von allgemeinen epistemologischen Überzeugungen (Trautwein \& Lüdtke, 2004) verwendet werden. Zum anderen soll eine Skala zu Lern-/Lehrbegriffen und Erziehungszielen (Bauer et al., 2009) eingesetzt werden, welche die drei Lernbegriffe „Pauken“, „Explorieren“ und „Verstehen“ - sowie ,instruktionsorientierte“ und „lernbegleitungsorientierte“ Lehrbegriffe erfasst. Bei Erziehungszielen werden zum Beispiel „Leistungs- und Anstrengungsbereitschaft“ oder „,vielseitiges Wissen“ thematisiert. Mittels korrelativer Zusammenhänge mit diesen inhaltsnahen Variablen wird die Konstruktvalidität des neu entwickelten Fragebogens überprüft. Zudem soll die Sensitivität des Instruments gegenüber geschlechts- und fächerkombinationsspezifischen Unterschieden in den Überzeugungen getestet werden. Die Fragestellungen beziehen sich demnach auf die faktorielle Struktur des Fragebogens, dessen Reliabilität und Konstruktvalidität:

1) Lassen sich die vier Überzeugungen zum Lehren und Lernen und/oder ihre Facetten mithilfe eines quantitativen Messinstruments erfassen? Lässt sich die 4-FaktorenStruktur mittels einer exploratorischen Faktorenanalyse bestätigen? Sind die gebildeten Skalen reliabel?

2) Lassen sich die zu erwartenden Zusammenhänge zwischen den Lehr-LernÜberzeugungen und den entsprechenden Lehr-Lern-Begriffen, Erziehungszielen sowie epistemologischen Überzeugungen mittels korrelativer Zusammenhänge mit den Skalen zu Lern-/Lehrbegriffen, Erziehungszielen und epistemologischen Überzeugungen wiederfinden? 
3) Lassen sich Unterschiede zwischen den mit dem Fragebogen ermittelten Werten in den Überzeugungen von Studentinnen und ihren männlichen Kommilitonen sowie Unterschiede zwischen Studierenden mit unterschiedlichen Fächerkombinationen ermitteln?

Verändern sich die Überzeugungen im Verlauf des Studiums? Zur Beantwortung der Frage nach der Bedeutung der vier Überzeugungen zu verschiedenen Zeitpunkten in der Lehrerausbildung werden Überzeugungen von Bachelor- und Masterstudierenden miteinander verglichen.

4) Lassen sich Unterschiede zwischen den Überzeugungen von Bachelor- und Masterstudierenden finden?

Aufgrund der Vermittlung von lehr-/lerntheoretischem Wissen im Studium kann eine Veränderung von Überzeugungen zum Lehren und Lernen in Richtung der Überzeugungen Konstruktion und Partizipation erwartet werden. 


\section{3 Überprüfung des Zusammenhangs zwischen epistemologischen und den lehr- /lernbezogenen Überzeugungen Transmission, Konstruktion, Partizipation und Schülerorientierung (Studie 3)}

Im Rahmen der dritten Studie soll der Zusammenhang zwischen epistemologischen und den vier lehr-/lernbezogenen Überzeugungen - Transmission, Konstruktion, Partizipation und Schülerorientierung - untersucht werden.

Ausgehend von Diskrepanzen zwischen den Operationalisierungen verschiedener Untersuchungen, die einerseits epistemologische Überzeugungen als Teil von lehr/lernbezogenen Überzeugungen (z.B. Dubberke et al., 2008) und andererseits die beiden Konstrukte gesondert behandeln (Chan \& Elliott, 2004; Cheng, Chan, Tang \& Cheng, 2009), wurde der Forschungsbedarf an weiteren Analysen des Zusammenhangs beider Konstrukte abgeleitet. Dahinter steht die Annahme, dass Lehrkräfte, die das Wissen als einfach und gesichert auffassen, eine rezeptive Überzeugung zum Lernen besitzen und Lehrkräfte, die das Wissen als ein Netzwerk von Theorien verstehen, der Überzeugung sind, dass Schülerinnen und Schüler ihr Wissen durch Verständigungs- und Aushandlungsprozesse selbst konstruieren (Olafson \& Schraw, 2006). Empirische Studien belegten zum Teil Zusammenhänge zwischen naiven epistemologischen Überzeugungen und traditionellen Lehr-Lern-Vorstellungen (Chen \& Elliott, 2004; Seidel et al., 2008), lieferten aber zum Teil auch andere, gegensätzliche Ergebnisse, zum Beispiel, dass fortgeschrittene epistemologische Überzeugungen mit traditionellen Lehr-Lern-Vorstellungen zusammenhängen können (Cheng et al., 2009). Daher sollen diese Zusammenhänge erneut überprüft werden.

Aufgrund des starken Interesses empirischer Forschung an der Transmission und Konstruktion wurden partizipatorische und schülerorientierte Lehr-Lern-Überzeugungen bislang wenig untersucht, sodass auch hinsichtlich des Zusammenhangs zwischen epistemologischen Überzeugungen und Partizipation sowie Schülerorientierung keine Erkenntnisse vorliegen. Deswegen soll diesem Zusammenhang in der dritten Studie der vorliegenden Arbeit nachgegangen werden. Daraus ergibt sich die folgende Fragestellung:

1) Gibt es einen Zusammenhang zwischen epistemologischen und den lehr/lernbezogenen Überzeugungen Transmission, Konstruktion, Partizipation und Schülerorientierung? 
Epistemologische und lehr-/lernbezogene Überzeugungen werden an einer Stichprobe von Lehramtsstudierenden erhoben. Zur Messung der vier lehr-/lernbezogenen Überzeugungen Transmission, Konstruktion, Partizipation und Schülerorientierung wird der neue, in der zweiten Studie bereits validierte Fragebogen Q4TB verwendet. Zur Erfassung von epistemologischen Überzeugungen wird die von Renkl und Gruber ins Deutsche übersetzte Version (nach Salhab, 2003) des Schommers Epistemological Beliefs Questionnaire (SEBQ) (Schommer, 1994b) Anwendung finden, welche neben der Kerndimension „Struktur von Wissen“ auch lernspezifische Überzeugungen wie Lernkontrolle und Lerngeschwindigkeit messen soll.

Die Ergebnisse bisheriger Forschung lassen einen Zusammenhang zwischen transmissiven lehr-/lernbezogenen und naiven epistemologischen Überzeugungen sowie zwischen konstruktivistischen lehr/-lernbezogenen und fortgeschrittenen epistemologischen Überzeugungen erwarten. Ausgehend vom theoretischen Hintergrund kann zudem ein Zusammenhang zwischen fortgeschrittenen epistemologischen Überzeugungen und der Überzeugung Partizipation vermutet werden. Diese Vermutung geht auf die Lerntheorie des Lerngemeinschaftsansatzes (Lave \& Wenger, 1991) zurück, nach welchem das Wissen als ein gemeinsames, im Prozess authentischer Kommunikation von den Mitgliedern der Lerngemeinschaft konstruiertes Produkt verstanden wird (Sfard, 1998; Lave \& Wenger, 1991). Daher wäre ein Zusammenhang zwischen partizipatorischen lehr-/lernbezogenen und fortgeschrittenen epistemologischen Überzeugungen denkbar. Dagegen dürften die Vorstellungen von Wissen für die Überzeugung Schülerorientierung weniger von Bedeutung sein. In erster Linie handelt es sich bei der Schülerorientierung um die Schülerpersönlichkeit mit ihren Bedürfnissen nach Autonomie, Kompetenzerleben und sozialer Eingebundenheit, sodass motivationale und nicht kognitive Aspekte des Lernens im Fokus stehen.

2) Unterscheiden sich die lehr-/lernbezogenen Überzeugungen in Abhängigkeit von den Zeitpunkten in der Lehrerausbildung?

Mit dieser Frage wird dem zweiten Anliegen der vorliegenden Arbeit nachgegangen, und zwar der Bedeutung verschiedener Lehr-Lern-Überzeugungen in Abhängigkeit vom Zeitpunkt in der Lehrerausbildung. Im Rahmen der dritten Studie sollen die Überzeugungen der Lehramtsstudierenden am Anfang, in der Mitte und am Ende des Studiums miteinander verglichen werden. Ausgehend von der bisherigen Forschung sowie von den Ergebnissen der ersten beiden Studien werden auch hier Unterschiede erwartet. 


\section{$4 \quad$ Teachers' beliefs about learning and teaching at different stages of teachers' career (Study 1)}

\subsection{Abstract}

Teachers' beliefs play a significant role in classroom activities. Recent research primarily analyses transmission and construction, while other beliefs remain neglected. In addition, the present study considers participation and nurturing and it also investigates whether these beliefs are associated with different stages of teachers' careers. The fifty interviewees were student teachers at the (a) beginning, (b) middle, and (c) end of their studies, as well as (d) beginning and (e) experienced teachers. Content analysis indicates the prevalence of transmission beliefs. Student teachers at the beginning of their studies viewed teaching as nurturing and transmission, whereas student teachers in the middle of their studies had a focus on construction and participation. However, teachers with extensive professional experience have shown a belief pattern similar to beginning student teachers, thus returning to a preference for transmission. These results shed some doubts on the sustainability of beliefs taught in German teacher education. 


\subsection{Introduction}

Research on teachers' beliefs as assumptions about the learner, teacher, and learning and teaching process has a long history and remains a topic of interest. Beliefs influence interpretations of concrete situations (Calderhead \& Robson, 1991), and are therefore "the best indicator of the decisions individuals make throughout their lives" (Bandura, 1986; Dewey, 1933). There is a growing consensus in current research, that teachers' beliefs affect their own perceptions and consequently their practices in the classroom. This, in turn, influences the learning of their students and outcomes of students' learning (e.g., Calderhead, 1996; Fang, 1996; Peterson et al, 1998; Staub \& Stern, 2002).

In contemporary literature, however, there presents a vast array of divergent beliefs such as knowledge transmission, cognitive construction, participation in a learning community and nurturing of the student. It should be noted that the empirical research on teachers' beliefs focuses on differences between several groups, e.g., beginning student teachers or advanced teachers, regarding the variability or development of their beliefs. However, this research is often limited to a comparison of only two groups: Several studies have described the beliefs of student teachers (e.g., Calderhead, 1996; Wan Ng et al., 2010; Russel, 1988; Richardson, 1996), and compared beginning with graduate student teachers (Saban, 2010; Saban et al., 2007) as well as pre-service with in-service teachers (Martínez et al, 2001; Alger, 2009). Longitudinal studies in this field are a rarity, and even studies comparing more than just two groups are rare.

Therefore, this investigation aims is to contribute to a better understanding of teachers' beliefs regarding the different stages of teacher's career. The study was designed to address associations between different beliefs at three stages of teacher education, and two levels of teaching experience. To specify different beliefs, the interviews were analyzed with regards to which beliefs already described in the literature could be distinguished on the basis of the interviews. Compared with questionnaires, the interview approach seems to examine the personal view more closely. The interviews were initiated by means of a self-drawn picture of the interviewees, following a study by Alexander and Dochy (1995). Thus, gained information largely approximates implicit knowledge of the participants and revives the subjective teaching experiences. Generally, the qualitative approach enables eliciting beliefs of the interviewees without copying an artifact of a quantitative questionnaire, whereas the quantification of the interview data allows a comparison of the beliefs at different stages of a teacher's career regarding the influence of teacher education and teaching experience. This 
mixed approach can be seen as a strong point of this study as it conflates the advantages of both methodologies and seems to avoid their disadvantages to a great extent.

The interviews were conducted in the context of a secondary school with student teachers studying at a large university. This is responsible for teacher education in one state in Germany as well as for beginning and experienced teachers from secondary schools (academic track; germ. Gymnasium) from the university's city.

A better understanding of the teachers' beliefs throughout their teaching career is important for both students' learning and their achievement; it could also contribute to the success of teacher education (van Driel et al., 2007). As the impact of teacher education has been criticized (Cochran-Smith \& Zeichner, 2005; Grossman, 2008) and comprehensive reform of teacher education has reached international dimensions, the investigation of teachers' beliefs during educational studies and throughout the teaching career is of immense relevance for teacher education and professional development of teachers today. 


\subsection{Theoretical background}

In the present study, teachers' beliefs are defined as personal and experience-based assumptions about the learner, the teacher and the learning and teaching process.

Teachers' beliefs about learning and teaching do not change easily or rapidly, because they are at the core of teachers' world-view (Pajares, 1992). Many researchers spoke in favor of resistance-to-change of beliefs, especially if they distinguished beliefs from knowledge. Beliefs are more inflexible and less dynamic than knowledge (Nespor, 1987).

Belief systems do not require general or group consensus regarding the validity and appropriateness of their views. Individual beliefs do not even require internal consistency within the belief system. Sometimes individuals tend to convert conflicting evidence into support for already held beliefs, using whatever cognitive tricks are necessary (Nisbett \& Ross, 1980; Tillema, 1998).

Teachers' beliefs are seen to be evaluative (Nisbett \& Ross, 1980) and affective (Nespor, 1987). They influence the decisions individuals make throughout their lives (Bandura, 1986; Dewey, 1933). Martínez et al. (2001) uses the metaphor "blueprint of thinking" (Martínez, et al, 2001, p. 965) to illustrate the nature of the "teachers' tacit theories" (Martínez, et al, 2001, p. 965), as they generally could be understood as dispositions to action.

\subsubsection{Different beliefs}

Theoretical paradigms of research on learning and teaching serve as a frame for different beliefs about learning and teaching. They will be described in the following section.

Since the rise of cognitive psychology, attention has been paid to the juxtaposition of two opinions; follow for example the debate between Anderson, Reder, and Simon (1997) and Greeno (1997). This debate encompassed the opposition of the associationist versus cognitive constructivist theoretical paradigms. The associationist theory, also known as behaviorism, dominated the first part of the 20th century, and at present continues to shape the culture of schooling (Resnick \& Hall, 1998). From this theory, the direct transmission view of learning and teaching has been derived. This view represents teaching as the transmitting process of information from the teacher to the learner, and learning as acquisition of stable, simple and independent knowledge pieces (Sfard, 1998). In contrast, a cognitive construction view, which evolved primarily from Piaget's work on cognitive development, describes learning as the individual's own active cognitive process which is influenced by the student's prior 
knowledge and environment (e.g., Bereiter, 1994). This perspective on learning focuses on the learning process rather than the outcomes of these processes. Some authors have made a kind of grading of these two theories by designating them as "traditional" and "progressive" paradigms, respectively (e.g., Handal, 2003; Meirink et al., 2009). Additionally, since the mid 1990s there have been clear signs within the context of research on teaching and learning for a consensus regarding the value of constructivism for didactics (e.g., for mathematics: de Corte, 2004; Treagust, Duit \& Fraser, 1996). Furthermore, empirical studies have already shown that the cognitive construction view, which deals with the cognitive activation and constructive support of the learners, seems to be more promising in promoting a deeper understanding of students' learning in mathematics than the direct transmission view (Aguirre \& Speer, 2000; Dubberke et al., 2008; Staub \& Stern, 2002).

Nevertheless, there are authors who are calling for plurality when looking at the beliefs about teaching and learning. Sfard (1998) pointed out the "dangers of choosing just one" (p. 4) of the two metaphors. Additionally, Pratt (2002) warned against the "one size fits all" (p. 1) notion of good teaching. For this reason, Sfard (1998) campaigned for participation, which was introduced as Situated Learning (Brown, Collins \& Duguid, 1989; Greeno, 1997) with its learning approaches Community of Practices (Lave \& Wenger, 1991) and Apprenticeship (Collins et al., 1989; Palincsar \& Brown, 1984; Rogoff, 1990; Scardamalia \& Bereiter, 1987). All these approaches stress the social dimension of learning. Thus, learning from this perspective has been seen as a process of becoming a member of a certain community, including the ability to act according to its particular norms (Sfard, 1998). It is based on dialogue exchanges, authentic activity and supportive collaboration between community members (Martínez et al. 2001; Pratt, 2002). Nevertheless, only a few studies have investigated this perspective: as establishing interpersonal relationship in a study by Dunkin and Precians (1992), teaching as engaging in community in a study by Alger (2009) as well as a situative/sociohistoric view in a study by Martínez et al. (2001) and by Leavy et al. (2007).

The other belief places learner's personality at the center of consideration. To date, very few authors have described nurturing in detail: Alger (2009) as a metaphor; Pratt (2005) as a perspective; Kember (1997) as the student-teacher-interaction; Dunkin and Precians (1992) as establishing interpersonal relationship. The nurturing belief reflects the motivation theory of learning with psychological needs (Deci \& Ryan, 1987; 1993), the learning approach of a supporting learning atmosphere (Klieme et al., 2006), and also pedagogical learning theory humanism (Huitt, 2009; Rogers, 1969; Rogers \& Freiberg, 1994). Similarly, 
in Oser's $(1998,2009)$ professional teachers' standards, the aspects of student-teacherrelations have been linked, as the ethics of the profession, with caring and justice. Assuming that each individual can develop all their innate potential capabilities to their maximal potential, nurturing interprets learning as the growth of a learner in a convenient environment for development (Mahlios \& Maxson, 1998; Oxford, Tomlinson, Barcelos, Harrington, Lavine, Saleh et al, 1998). Such an environment has an atmosphere without fear of failures and/or "emotional wounds" (Pratt, 2005, p. 164); it respects the psychological needs of the student for social interaction, experience of their own competence, as well as satisfactory social contacts (Deci \& Ryan, 1987, 1993).

Although the contents of the two beliefs, participation (Alger, 2009; Dunkin \& Precians, 1992; Leavy et al., 2007; Martínez et al., 2001) and nurturing, (Brookhart \& Freeman, 1992; Calderhead, 1996; Richardson, 1996; Russel, 1988) have been described in qualitative studies with beginning student teachers, there is still a lack of research regarding these two beliefs. Empirical quantitative research, especially with a focus on students' achievements as a result of specific teachers' beliefs, has concentrated so far on the transmission vs. construction views (e.g., Dubberke et al., 2008; Staub \& Stern, 2002). Furthermore, research focuses typically on patterns in pairs: transmission vs. construction views (e.g., Staub \& Stern, 2002; OECD, 2009), content-centered vs. learning-centered views (van Driel et al., 2007; Kember \& Kwan, 2000). For this reason, the research needs to consist of the investigation of several beliefs. Therefore, in addition to transmission and construction, the present study also takes the beliefs, participation and nurturing, into account.

\subsubsection{Variability of beliefs about learning and teaching}

The question regarding the development or variability of teachers' beliefs still evokes controversy. The results depend on the investigated groups. Thereby, most of the studies focus only on one group, as well as describing its beliefs. In the following, the studies will first be presented, with beginning student teachers when focusing on the duration of educational studies and will then be followed by comparison studies of several groups.

Studies focusing on beginning student teachers found that student teachers frequently start their studies with views of teaching as telling (Calderhead, 1996; Russel, 1988) and as a mechanical transfer of information (Brookhart \& Freeman, 1992; Richardson, 1996), wherein the teacher hands down knowledge to the children, and learning involves memorization of material (Black \& Ammon, 1992). Beginning student teachers mentioned caring, 
understanding, warmth, and being friendly with the ability to relate to children as characteristics of a good teacher (Rathbone \& Pierce, 1990; Weinstein, 1989). Brookhart and Freeman (1992) found in their review that "entering teachers view the nurturing and interpersonal aspects of a teacher's role as more important than academic aspects" (p.51). Results of the current study (Wan Ng et al., 2010) also indicated that in the opinion of the preservice teachers "building relationships with the students" are an important characteristic of a good teacher. It could be expected that the beliefs of student teachers, at the beginning of their studies, consist of ideas about teaching as transmission, affective and interpersonal aspects of the relationships with their students.

However, the beliefs about learning and teaching seem to be variable as previous research indicates a shifting away from the nurturing aspects of teaching in the beliefs of the student teachers during their educational studies. Nettle (1998) found evidence of a change from affective to task oriented beliefs; Rust (1994) mentioned the shifting of the beliefs from helping to managing in classrooms. Studies focused on teacher education mentioned insufficient development of beliefs (see reviews by Nettle, 1996; Wideen et al., 1998) as the existing beliefs of beginning student teachers are fairly robust and act as filters through which teacher education programs are viewed (Kagan, 1992; Pajares, 1992; Weinstein, 1990). Thus, Wideen et al. (1998) stated in his review that beliefs were resistant to short-term intervention. For example, Weinstein (1990) reported on the lack of changes after a seven-week course combined with fieldwork experience. Richardson (1996, 2003) even referred to teacher preparation programs as the "weak intervention" of a few years. Moreover, what is learned during teacher education "washes out" once pre-service teachers leave the university and become socialized in the field (Kagan, 1992; Zeichner \& Liston, 1987).

On the other side, there are several studies referring to differences in student teachers' beliefs. A comparison study by Saban, Kocbeker \& Saban (2007) described varying metaphorical teacher images of beginning student teachers and high-semester student teachers: The view of a teacher shifted from "growth-oriented" (e.g., gardener) toward "facilitation-oriented" (e.g., lighthouse). These metaphorical images remain contents of nurturing and construction beliefs of learning and teaching respectively. Hollingsworth (1989) presented comparable results from his longitudinal study: He reported on the development of beliefs during the fifth-year teacher educational program towards a constructivist view. The current study by Leavy et al. (2007) also refers to a change in beliefs among student teachers from behaviorist toward constructivist, metaphorical representations. Saban reported contrary results (2010): The prospective teachers in his study became more transmission- and less 
constructivist-oriented in their learner-images. However, all these longitudinal studies understand changes in beliefs as a result of educational experience.

The developmental approach explains changes in the beliefs, which are described as part of student teachers' development: Marton, Dall'Alba, \& Beaty (1993) referred to several development stages in the thoughts of university students from the view of learning as an increase of knowledge by memorizing, to learning as seeing something in a different way (Marton et al., 1993). The study by Morgan and Beaty (1997) supported this suggestion as they found learning concepts which spanned a six year period, according to the developmental stages described by Marton et al. (1993).

Studies focusing on practicing teachers provided evidence for differences between novice and experienced teachers, as well as for a change of beliefs throughout their teaching career. Martínez et al. (2001) compared the metaphorical images of experienced teachers with prospective teachers. The majority of metaphors $(57 \%)$ formulated by experienced teachers depicted behaviorist metaphors, which were much higher than metaphors used by prospective teachers $(22 \%)$. On the other hand, prospective teachers utilized more constructivist metaphors $(56 \%)$ than experienced teachers did (38\%). Compared to experienced teachers (less than 2\%), prospective teachers, more often, depicted teaching and learning as a social process, with learning as an integral part of the social activities of a community and knowledge as distributed inside of it (about 20\%). In Alger's survey (2009), the practicing teachers selected the presented metaphors of learning and teaching such as transmitting, nurturing, and engaging in a community. This was done regarding the question: which of these metaphors were more likely, in their view about learning and teaching at different stages of their career. The results showed that the majority of the teachers $(60 \%)$ perceived changes in their beliefs. Additionally, the change in direction seemed to depend on the teaching experience. Teachers with many years in their job stated that they began their careers with a transmissive, teacher-centered view and later changed this opinion toward a learner-centered view. The other way around, teachers with less experience stated the opposite; they began their careers with learner- centered views on learning and teaching. Then they started to understand teaching and learning increasingly as teacher-centered. Thus, the teaching experience seems to be an important element for the development of beliefs about learning and teaching.

It can be summarized that current research regarding beliefs over the teaching career provides evidence for variations in beliefs. Differences between beginning and high-semester student teachers were reported against differences between beginning and experienced 
teachers. However, some of the studies presented above have methodological limitations. For example, Alger (2009) asked her participants to envision their former views on learning and teaching. Furthermore, these studies are limited to two groups of teachers; consequently, there are still no studies which analyse more than two stages of the teaching career. The findings of studies allow for statements about beliefs of particular teacher groups, such as beginning student teachers or novice teachers, but were conducted in different contexts, and used different methodological approaches. Moreover, some of these studies address a single aspect of learning and teaching, such as "the teacher" (Saban et al., 2007) or "learning" (Saban, 2010); furthermore, they stay on a descriptive level, or use metaphors for investigation of the beliefs (Martínez et al., 2001). A study investigating several aspects of beliefs as well as several stages of the teaching career would have methodological advantages and allow statements about several stages of the teaching career. The purpose of the present study was to analyze several aspects of beliefs about learning and teaching, and to compare teachers at different stages of their careers (student teachers at the beginning, in the middle and at the end of teacher education and beginning and experienced practicing teachers). 


\subsection{Research questions and hypothesis}

The aims of the present study were: (1) to analyze whether, and to what extent, the beliefs already described in previous research would be addressed in the self-reflection about learning and teaching of the interviewees; (2) to investigate to what extent teachers, at different career stages (student teachers at the beginning, in the middle, at the end of their teacher education as well as beginning and experienced teachers) differ with regards to their beliefs about learning and teaching. Two well-defined beliefs, transmission and construction, were identified (e.g., Staub \& Stern, 2002; Dubberke et al., 2008) from the literary overview. However, some other conceptions were found which address differing aspects of them. Participation (Sfard, 1998) emphasizes social aspects of learning; and nurturing, whereas according to Pratt et al. (2005), it stresses a learner's personality. Other studies analyzed conceptions congruent with either these four aspects or with only a single aspect (e.g., Alger, 2009; Martínez et al, 2001; Trigwell \& Prosser, 2004).

Keeping in mind these different beliefs, the first research question focused on the extent to which these beliefs were addressed in the interviews. Therefore, it was expected to extract transmission and construction from the interviews as these beliefs were identified in most of the studies focusing on teachers' beliefs. Similarly, it was hypothesized that transmission would be addressed more often than other beliefs, because behaviorism, as the theoretical background of transmission, is the one with the longest traditions in research. Compared with constructivism, for instance, its ideas seem to be better known among the wider public. More important, however, there were no expectation limits to these two beliefs so that the question was whether ideas of participation or nurturing would be included in the beliefs of the interviewees.

The second research question focused on the different stages of a teaching career. It was expected to find differences in beliefs with regards to the three stages of teacher education as well as to the two levels of teaching experience. The first hypothesis was that beginning student teachers would hold a transmissive view on teaching, according to the reported view of teaching as telling (Calderhead, 1996; Russel, 1988) and transfer of information (Brookhart \& Freeman, 1992; Richardson, 1996). The second hypothesis had to do with nurturing. This belief was expected to occur among beginning student teachers, as described in previous studies (Brookhart \& Freeman, 1992; Wan Ng et al., 2010; Rathbone \& Pierce, 1990; Weinstein, 1989). Third, differences between the beliefs held by beginning and advanced student teachers were expected. According to previous studies from the field of 
teacher education, as social constructivism builds a central component of contents of teacher educational programs in German universities, it could be hypothesized that the belief of student teachers in the middle and at the end of their studies would include more ideas of constructivism and participation (Leavy et al., 2007; Saban, 2010; Saban et al., 2007). Finally, differences between student teachers and practicing teachers were expected. According to the results of the study by Martínez et al. (2001) experienced teachers were expected to address more ideas of transmission compared with prospective teachers - student teachers, who would prefer construction and participation. Moreover, the more influential critics of teacher education (e.g., Grossman, 2008) supported these expectations on teachers' behavior. 


\subsection{Method}

\subsubsection{Participants - design}

The sample $(N=50)$ consisted of student teachers (mean age $=23.73 ; S D=4.34)$ (a) at the beginning (mean age $=20.9 ; S D=1.10$ ) (mean studied semesters $=2.2 ; S D=.63$ ), (b) in the middle (mean age $=23.10 ; S D=.74)$ (mean studied semesters $=7.0 ; S D=.00$ ), (c) at the end of their studies (mean age $=27.20 ; S D=6.01$ ) (mean studied semesters $=9.5 ; S D=$ 1.90 ), (d) beginning (mean age $=28.4 ; S D=3.37$ ) (mean teaching experience $=10.4$ months, $S D=5.74$ ), and $(\mathrm{e})$ experienced teachers (mean age $=48.8 ; S D=8.40$ ) (mean teaching experience $=17$ years; $S D=9.74)$. Thus, a one-factorial between-subject design was used which was comprised of the five groups with ten participants, respectively. The participants of the first three groups were student teachers in their teacher education programs at a German university. They differed from one another in their knowledge gained through their studies, and practical experiences, as student teachers in the middle and at the end of their studies had to do the obligatory internships at the schools during their educational studies, whereas student teachers at the beginning of their studies had not yet done so. Mathematics, physics, history, biology and languages were the disciplines studied by the student teachers and taught by the practicing teachers in the sample. The distribution of disciplines was balanced, as both discipline groups were announced - sciences and humanities. Aside from this, it was not the purpose of the present study to compare different disciplines due to the sample size.

\subsubsection{Interview procedure}

In this study, an interview approach was used to examine student teachers' and practicing teachers' beliefs about learning and teaching. To facilitate the reflection and articulation about beliefs, first the interviewees were asked to draw their ideas about learning and teaching following a study by Alexander and Dochy (1995). The drawings were limited to 15 minutes. The time was sufficient for all participants to finish the picture. Then, the participants explained their picture to the interviewer, describing their ideas of learning and teaching. The interviewer asked questions about unaddressed parts of the pictures as well as ambiguous statements to get agreement on proper understanding. Each interview took about one hour. The interviews were recorded and transcribed. All interviews were codenamed coded to ensure the anonymity of the data. 


\subsubsection{Interview analysis}

Analysis of the interviews proceeded in a manner consistent with the quantifying, qualitative analysis according to Chi (1997).

\section{Segmenting process}

As preparation for the coding process, the interview transcripts were first segmented into single coding units as Chi (1997) suggested. A segment was defined as a single idea unit that could be an initial mention of an idea, a synonym of this idea, an example of a statement, a single point in a list of features of a subject, as well as one reasoning chain in explanation or in argumentation speech. Some examples for such segmenting rules with an anchored example of a segment follow:

A single segment is a repeated mention of an aspect to affirm its importance: ...also important, I think, is discipline in the classroom//

A single segment is an additional explanation of this aspect: ...it means the teacher marks out the framework in which the students can range freely//

\section{Developing a category scheme}

In the next step of the analysis, a coding scheme was constructed based on the theoretical rationale sketched out in section 2.1.5. As a result, the main categories were developed a priori and represented the four beliefs transmission, construction, participation and nurturing. Additionally, differentiation categories were defined on the basis of the interviews. These categories represented the subordinate categories of a main category: that means the details and single ideas of a particular belief according to the previous research regarding beliefs, such as "a passive role of a student," of the belief transmission. Thus, the coding process was partly deductive and partly inductive. In this way, the analysis answers the questions of whether and to what extent the beliefs already described in previous research were addressed in the interviews.

Development and refinement of the coding scheme were done by two researchers. The computer program MAXQDA 10 (MAXQDA, 1989 - 2012) was used to support the coding process of the verbal data. The coding process was divided in a training phase and a coding 
phase. In the training phase, cases of disagreement were discussed until a consensus was found. That way, calibration of the coding process was achieved. As a result of this process, twenty-eight differentiation categories were obtained. In the coding phase, both raters assigned the single ideas to these differentiation categories independently of each other. The second rater analyzed fifteen of the twenty-five interviews. The inter-rater reliability, as determined by Cohen's Kappa, was good $(\kappa=0.73)$.

The next step of the analysis was deductive, which means that the contents of the four beliefs were obtained from the previous literature. That way, the differentiation categories were examined for coherence to one of the four main categories and thus represented the details and single ideas of the four beliefs literature. Occurring divergences were discussed with researchers and experts from the department. However, some additional categories remained unclassified, as they could not be assigned to a main category. Instead they express the themes which were not the object of the present study. Table 2 shows some examples of differentiation categories as well as an example of a segment for each belief (see Table 2).

Table 2

Examples of differentiation categories and segments for each belief and for non-classified statements

\begin{tabular}{|c|c|c|}
\hline Belief & $\begin{array}{l}\text { Examples of a differentiation } \\
\text { category }\end{array}$ & Examples of segments \\
\hline Transmission & $\begin{array}{l}\text { Knowledge transmission by the } \\
\text { teacher }\end{array}$ & $\begin{array}{l}\text { I have knowledge, which I want to convey } \\
\text { to my student }\end{array}$ \\
\hline Construction & Learning as the active process & $\begin{array}{l}\ldots \text { the student has to build on his/her own, } \\
\text { what s/he needs; s/he seeks information on } \\
\text { his/her own, which is interesting according } \\
\text { to his/her opinion. }\end{array}$ \\
\hline Participation & $\begin{array}{l}\text { Common learning in the } \\
\text { learning community }\end{array}$ & $\begin{array}{l}\text { You can achieve more in a group than } \\
\text { alone, because one student masters this } \\
\text { thing better than others, and the other } \\
\text { student can do other things better... }\end{array}$ \\
\hline Nurturing & $\begin{array}{l}\text { Strengthening of student's self- } \\
\text { efficacy by the teacher }\end{array}$ & $\begin{array}{l}\text { When a student feels they are accepted, } \\
\text { acknowledged, then the student's self- } \\
\text { efficacy will strengthen and s/he will feel } \\
\text { comfortable, and therefore learn better. }\end{array}$ \\
\hline Non-classified & $\begin{array}{l}\text { Teachers from the family as } \\
\text { reference }\end{array}$ & $\begin{array}{l}\text { My mum - she is a teacher in an } \\
\text { elementary school - always says that... }\end{array}$ \\
\hline
\end{tabular}




\subsection{Results}

\subsubsection{The four beliefs}

The first research question was whether, and to what extent, the beliefs already described in previous research would be addressed in the self-reflection phase about learning and teaching of the interviewees. The content analysis was guided by the beliefs transmission, construction, participation and nurturing as these beliefs were identified in the literary overview in anticipation of diverse beliefs.

In the coding process, the single segments were assigned to the differentiation categories of these four deductively developed main categories. The following four beliefs were addressed in the interviews:

(a) knowledge transmission, according to which teaching is transmitting knowledge from the teacher to his students;

(b) cognitive construction, which described learning as an active, constructive process, influenced not as much by the teacher, but rather by prior knowledge and interests of a student;

(c) participation with an understanding of learning as a common process within a learning community and

(d) nurturing, according to which the student's personality with its psychological needs such as the need for competence, autonomy, and relatedness (Deci \& Ryan, 1987, 1993 ) is to be respected by the teacher.

To interpret the data, a number of segments was counted as well as a number of differentiation categories according to a particular belief. In doing so, the view of a particular belief was obtained without regard to the interviewees. This view answers the questions: To what extent was a particular belief addressed in the interviews? How many single ideas were mentioned regarding it? Table 3 demonstrates the frequencies of the single segments and differentiation categories according to a particular belief. 
Table 3

Frequencies of categories, segments and average segments in each interview for each belief

\begin{tabular}{llll}
\hline Belief & $\begin{array}{l}\text { Category: } \\
\text { frequency }\end{array}$ & $\begin{array}{l}\text { Segments: } \\
\text { frequency }\end{array}$ & $\begin{array}{l}\text { Average segments in } \\
\text { each interview } \\
\text { (mean-max) }\end{array}$ \\
\hline Transmission & 12 & 1334 & $26.68(1-72)$ \\
Construction & 7 & 1155 & $23.10(1-62)$ \\
Participation & 4 & 1056 & $21.12(4-52)$ \\
Nurturing & 5 & 789 & $15.78(0-45)$ \\
Non-assigned & 5 & 305 & $6.10(0-43)$ \\
\hline
\end{tabular}

As this table shows, the beliefs received different counts of categories and segments. The belief transmission consisted of twelve differentiation categories with 1334 single segments. It means that the interviewees had stated 1334 single utterances regarding transmission, and that there were twelve single ideas regarding transmission in the interviews. The interviewees addressed seven single ideas regarding construction as well as four regarding participation with 1155 and 1056 segments, respectively. On average, each interviewee made 26.68 utterances regarding transmission, 23.10 regarding construction, 21.12 regarding participation, and 15.78 regarding nurturing. This view of the data shows that transmission is the belief with the highest count of single segments as well as the highest count of categories. Thus, the first research question could be answered as follows: According to our expectation, the beliefs knowledge transmission and cognitive construction were found in the participants' interviews. In addition, participation and nurturing were identified in the self-reflection. However, these beliefs differed in their extent. The beliefs transmission showed it prevailed when compared with construction, participation and nurturing.

\subsubsection{Differences between different stages of teacher career regarding beliefs about learning and teaching}

The second research question referred to the extent to which teachers, at different career stages, differ with regard to their beliefs about learning and teaching. Five groups of (student) teachers were investigated: a) the student teachers at the beginning, b) in the middle, c) at the end of their studies and d) beginning and e) experienced teachers. To answer this 
question the counts of segments regarding single categories were entered into the SPSS program (IBM SPSS Version 19). As already mentioned above, there were some unclassified categories. They were excluded from the following analysis because they did not address learning or teaching.

The first analysis focused on the data regarding the preference of the participants for a single belief: How many participants had one, if any, preferred belief? The so-called "dominant belief", determined as the belief that has a value of one standard deviation above the individual mean, was calculated for each participant. Table 4 shows the number of participants holding a particular belief as their dominant belief.

Table 4

Numbers of participants with one belief as their dominant belief

\begin{tabular}{lcccccc}
\hline & $\begin{array}{l}\text { Student } \\
\text { teachers } \\
\text { at the } \\
\text { beginning } \\
\text { of their } \\
\text { studies } \\
(\mathrm{n}=10)\end{array}$ & $\begin{array}{l}\text { Student } \\
\text { teachers } \\
\text { in the } \\
\text { middle of } \\
\text { their } \\
\text { studies } \\
(\mathrm{n}=10)\end{array}$ & $\begin{array}{l}\text { Student } \\
\text { teachers } \\
\text { at the end } \\
\text { of their } \\
\text { studies } \\
(\mathrm{n}=10)\end{array}$ & $\begin{array}{l}\text { Begin- } \\
\text { ning } \\
\text { teachers } \\
(\mathrm{n}=10)\end{array}$ & $\begin{array}{l}\text { Experi- } \\
\text { enced } \\
\text { teachers } \\
(\mathrm{n}=10)\end{array}$ & $\begin{array}{l}\text { Whole } \\
\text { sample }\end{array}$ \\
\hline Transmission & 4 & 0 & 1 & 2 & 5 & 12 \\
Participation & 2 & 2 & 1 & 2 & 1 & 8 \\
Construction & 1 & 2 & 3 & 0 & 0 & 6 \\
Nurturing & 3 & 0 & 1 & 1 & 1 & 6 \\
\hline
\end{tabular}

The results show that 32 of 50 participants had a dominant belief. Accordingly, transmission was dominant for 12 participants, participation for eight, construction for six, and nurturing also for six. The groups differed in the number of the dominant belief. Again, transmission revealed its prevalence: two of five groups, as well as the whole sample, had this belief as the first position in their ranking list: the beginning student teachers and the experienced teachers. The student teachers in the middle of their studies preferred participation and construction and no other belief at all. The student teachers at the end of their studies had construction in the first position of this ranking list; the beginning teachers two beliefs transmission and participation.

Second, the counts of single segments for the four beliefs were compared for different groups. Figure 2 displays the results. 


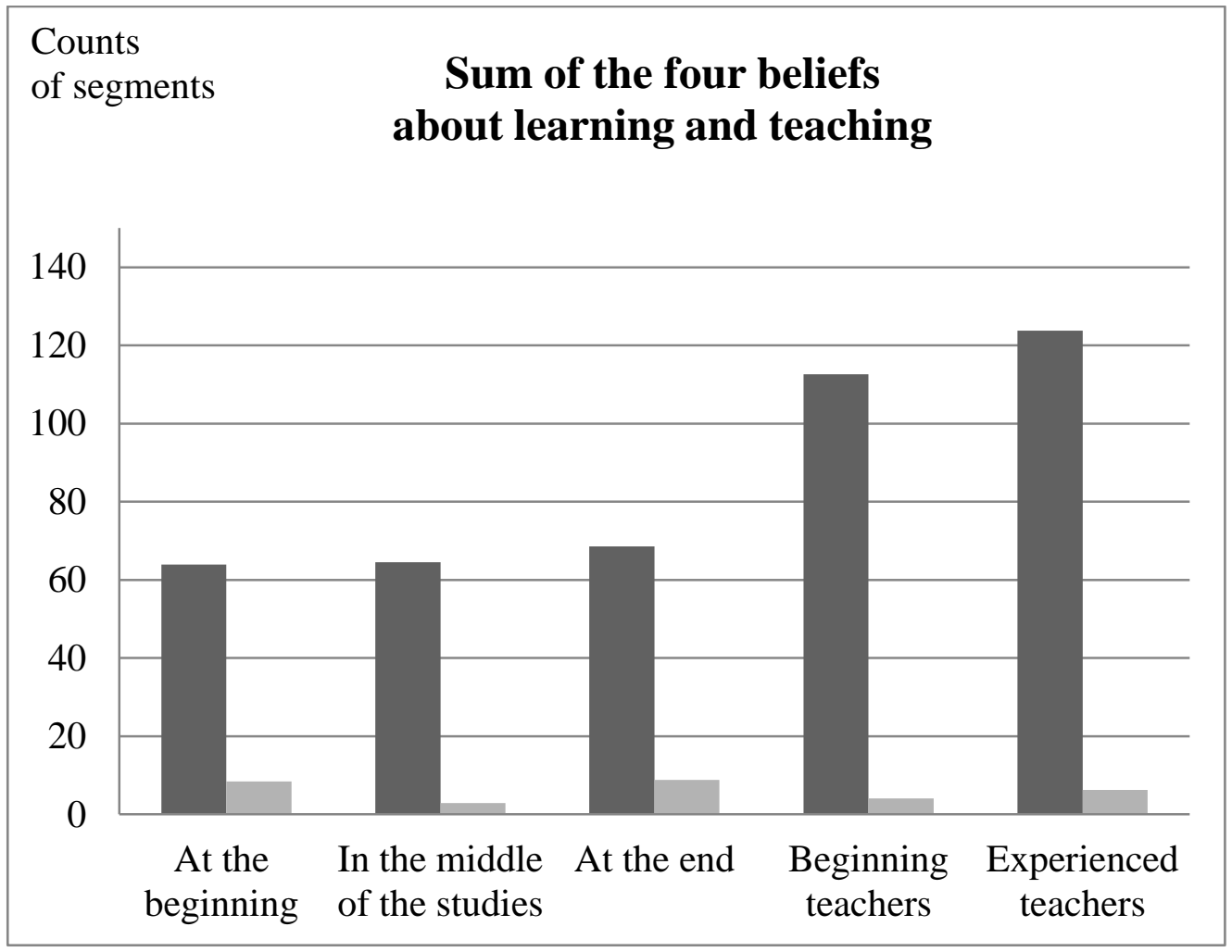

Figure 2. Counts (means) of the segments regarding learning and teaching for the five groups of interviewees.

The more advanced the interviewees were in their educational studies and teaching careers, the more they referred to learning and teaching. Statistical analysis was performed by means of one-way analysis of variance (ANOVA) for the sum of the segments regarding the four beliefs as dependent variables by the teaching career stage as group factor. The difference between the five groups was significant, $F(4,45)=8.49, p<.001, \eta 2=.43$. To identify the significant differences between the groups, a post hoc Tukey analysis was conducted. The results showed significant differences between all three groups of student teachers and both groups of teachers. The student teachers at the beginning of their studies differed from both the beginning teachers $(M=-48.70, p<.05)$ and the experienced teachers $(M=-59.90, p<.05)$. The student teachers in the middle of their studies differed from the newly qualified teachers $(M=-48.10, p<.05)$ as well as from the experienced teachers $(M=$ $-59.30, p<.05)$. However, the student teachers at the end of their studies differed from the newly qualified teachers $(M=-44.00, p<.05)$ as well as from the experienced teachers $(M=$ $-55.20, p<.05)$.

Third, the counts of segments were converted into percentages as they reproduce the relation of the segments regarding one particular belief to the other four beliefs (the sum of 
segments of the four beliefs equates 100 percent of statements about learning and teaching). Thus, the percentages represent the personal preference of the participants for a single belief. Table 5 demonstrates the means (in percentage) and standard deviations.

Table 5

Percentages of the four beliefs (means and standard deviation) for each group and the whole sample

\begin{tabular}{|c|c|c|c|c|c|c|}
\hline & $\begin{array}{l}\text { At the } \\
\text { beginning } \\
\text { of their } \\
\text { studies } \\
(\mathrm{n}=10)\end{array}$ & $\begin{array}{l}\text { In the } \\
\text { middle of } \\
\text { their studie } \\
(\mathrm{n}=10)\end{array}$ & $\begin{array}{l}\text { At the end } \\
\text { of their } \\
\text { studies } \\
(\mathrm{n}=10)\end{array}$ & $\begin{array}{l}\text { Beginning } \\
\text { teachers } \\
(\mathrm{n}=10)\end{array}$ & $\begin{array}{l}\text { Experi- } \\
\text { enced } \\
\text { teachers } \\
(\mathrm{n}=10)\end{array}$ & $\begin{array}{l}\text { Whole } \\
\text { sample } \\
(\mathrm{N}=50)\end{array}$ \\
\hline \multirow[t]{2}{*}{ Transmission } & 34.8 & 25.3 & 27.2 & 27.8 & 32.3 & 29.5 \\
\hline & (18.1) & $(9.5)$ & (15.1) & $(10.5)$ & (19) & (14.8) \\
\hline \multirow[t]{2}{*}{ Participation } & 21.6 & 31.4 & 26.9 & 24.9 & 23.8 & 25.7 \\
\hline & $(7.65)$ & (14.4) & (11.8) & (11.7) & $(9.8)$ & (11.3) \\
\hline \multirow[t]{2}{*}{ Construction } & 15.8 & 31.4 & 32.9 & 27.7 & 23.7 & 26.3 \\
\hline & (9.3) & (13.4) & $(10.2)$ & (7.5) & (7.6) & (11.3) \\
\hline \multirow[t]{2}{*}{ Nurturing } & 27.9 & 11.6 & 13.2 & 19.3 & 20 & 18.4 \\
\hline & (14.9) & (11.9) & (11.9) & $(10.7)$ & (14) & $(13.6)$ \\
\hline
\end{tabular}

The descriptive results comply with the expectations regarding the second research question. The student teachers at the beginning of their studies had higher values in transmission as well as in nurturing. Interestingly, the student teachers at the beginning of their studies commented on participation and construction remarkably less. However, the corresponding theories of learning and teaching, which served as the framework for the beliefs construction and participation, belong to the contents of teacher education programs at German universities. That is why it was expected to find these two beliefs would be found in the middle and at the end of their studies. The expected differences between student teachers and practicing teachers were shown to be the following: Again, the experienced teachers stated more ideas of transmission than the student teachers in the middle and at the end of their studies. Figure 3 shows the distribution of the beliefs depending on the group. 


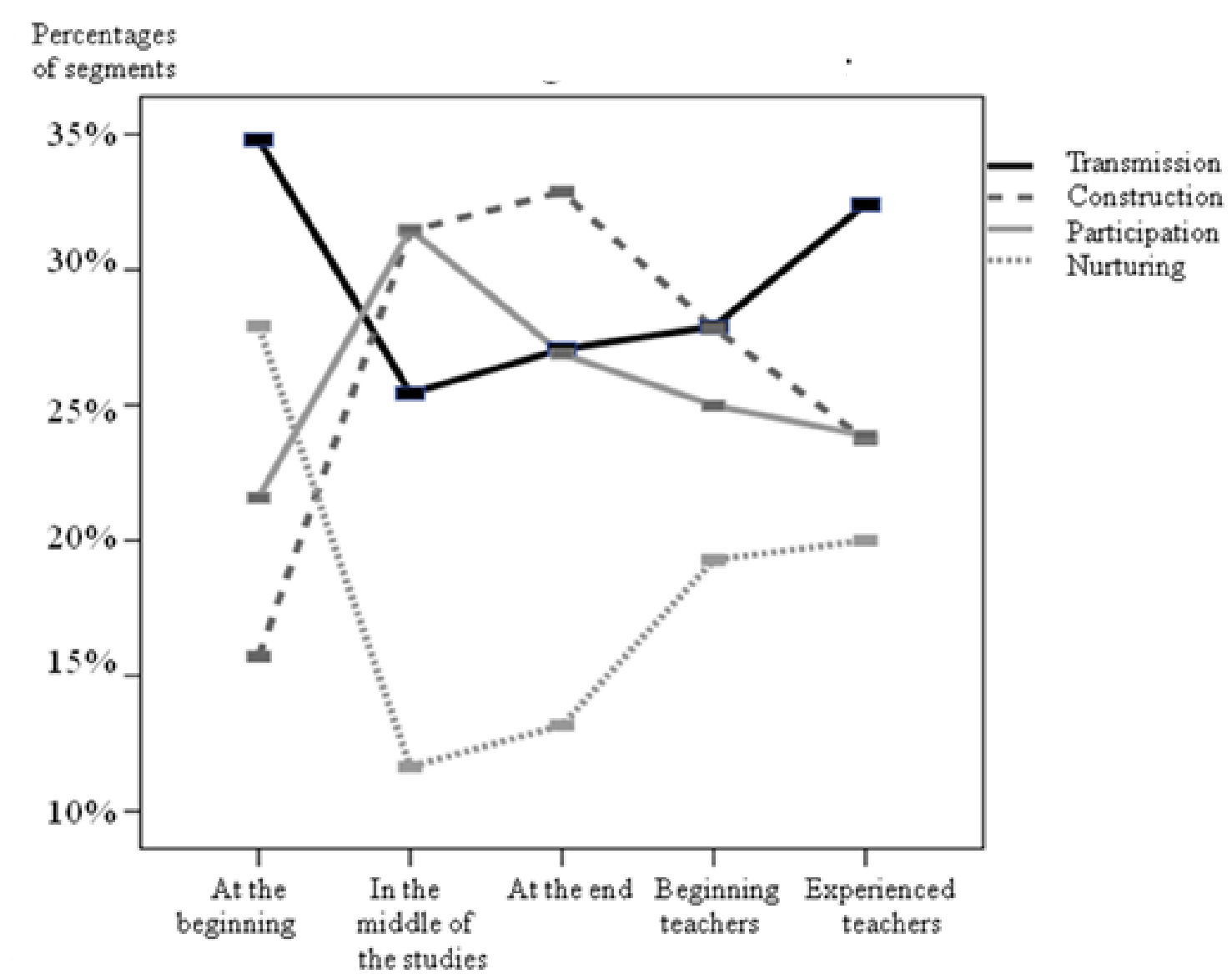

Figure 3. Distribution of the percentages of the beliefs for the five groups of the interviewees.

As Figure 3 shows, transmission was highest for student teachers at the beginning of their studies; it decreased in the middle and at the end of their studies, and increased once again for practicing teachers with growing experience. Construction and participation, however, were relatively low at the beginning of their studies, then became quite pronounced during their studies, but lost their prominence for practicing teachers, depending on the levels of experience. Nurturing was relatively prominent at the beginning, lower during the studies, and stronger with growing professional experience.

Statistical analysis was performed by means of multivariate analysis of variance (MANOVA) with "belief" as a within subject factor. The difference between the four beliefs was significant, $F(4,45)=6.65, p<.001, \eta 2=.37$. To identify the differences between the five groups, multivariate contrasts were defined for each belief as $2-1-2-12$ following the distribution showed in the Figure 2. This analysis tests the statistical significance of differences in the beliefs between the groups with univariate tests for each belief 
simultaneously. The results showed a significant difference in construction, $F(1,45)=15.54$, $p<.001, \eta 2=.26$, as well as nurturing, $F(1,45)=6.47, p<.05, \eta 2=.13$.

Evidently, there were different patterns of beliefs depending on stages of teacher education and levels of experience. This pattern confirms the hypothesis: Student teachers at the beginning of their studies viewed teaching as transmission and nurturing, whereas student teachers in the middle and at the end of their studies had a focus on construction and participation. Nevertheless, when commencing teaching, these beliefs began to decrease and with growing experience, teachers reclaimed a preference for transmission. However, the belief pattern of experienced teachers was similar to beginning student teachers in that both groups understand learning and teaching as transmission, and showed a preference for nurturing. In summary, the second research question could be answered as follows: The teachers at different career stages differed regarding their beliefs about learning and teaching; the differences in construction and in nurturing were particularly significant. 


\subsection{Conclusions and Discussion}

In analyzing teachers' beliefs about learning and teaching, the following beliefs were investigated: knowledge transmission, cognitive construction, participation and nurturing.

These beliefs have already been described in previous research, though insufficiently investigated. Previous studies have analyzed conceptions and views of learning and teaching similar to the four, but often only focused on a few aspects of some of them. For instance, most of the studies limited their research interest to the two beliefs: transmission and construction (e.g., Dubberke et al., 2008; Staub \& Stern, 2002), and seemed to consider the beliefs in pairs of contrast. Even the international OECD study "Teaching and Learning International Survey" (TALIS) focused on these beliefs (OECD, 2009). Other interview studies have identified several of the four beliefs with similar meanings (Martínez et al., 2001; Nettle, 1998; Saban et al., 2007; Weinstein, 1989). To date, there has been no study, which analyzed the four beliefs with several aspects described in previous theoretical and empirical literature.

In the present study, the diversity of beliefs about learning and teaching was kept in mind. Thus Pratt (e.g., Pratt, 1992, 2002, 2005) presented several perspectives on teaching among them were transmission, apprenticeship, developmental, and nurturing. Sfard (1998) also called for plurality in looking at the beliefs about teaching and learning, and pointed out the "dangers of choosing just one" of the two metaphors of learning and teaching (Sfard, 1998, p.4); similar to Pratt (2002), who warned against the "one size fits all" notion of good teaching (Pratt, 2002, p.5). Therefore, there were no expectation limits relating to the pair transmission and construction and as a result curiosity was sparked as to what extent the beliefs participation and nurturing would be addressed in the interviews. The results from the interview analysis can be summarized as follows.

From the content analysis of the interview transcripts, the conclusion can be drawn that the extent of the four analyzed beliefs transmission, construction, participation and nurturing was not the same. As predicted, transmission is more dominant than the other beliefs. Accordingly, the number of ideas, as well as of single segments regarding transmission, was higher than those for construction, participation and nurturing. Similarly, the interviewees preferred transmission to the other beliefs. Thus, the major implication of the present study is recognizing the prevalence of transmission compared with other beliefs.

When comparing the different stages of a teaching career, differences were found between teachers at different stages regarding their beliefs about learning and teaching. First, 
in line with previous studies, transmission and nurturing were highest at the beginning of educational studies (e.g., Brookhart \& Freeman, 1992; Calderhead, 1996; Richardson, 1996; Wan Ng et al., 2010); whereas construction and participation were relatively low. Second, the results of the present study support the results of previous research that found differences between beginning and advanced student teachers (Dahlgren \& Chiriac, 2009; Saban et al., 2007). In line with previous research (e.g., Hollingsworth, 1989; Leavy et al., 2007), construction was found to be quite pronounced during educational studies. In particular, the present study replicated the result of Martínez et al. (2001) showing a similar pattern for participation and construction. As predicted, a contrary pattern was found for nurturing and transmission. They decreased during the educational studies. These results were suggested by Nettle (1998) and Rust (1994), who had shown a shifting from affective and helping aspects in the beliefs of student teachers during their studies.

The popularity of transmission at the beginning of educational studies may be explained by the years of student experience from the tradition of rigidly structured schooling guided by the teacher. Similarly, the acquired university knowledge during the educational studies may be an explanation for the prominence of constructivism and participation.

Nevertheless, progressive beliefs such as construction and participation began to decrease when transition to school took place. With growing experience, transmission was again the most addressed belief. This result replicated that of Martínez et al. (2001) who had shown experienced teachers' preference for transmission. Obviously, the belief pattern of experienced teachers was similar to that of beginning student teachers. Therefore, although considerable effort has been made in the educational context the most traditional behaviorist theory- to put it in the words of Resnick and Hall (1998) -the view of learning and teaching still dominates and continues to shape the culture of schooling to the present day. That is the other major implication of the present study what should be discussed critically in the context of teacher education. 


\subsection{Implication for practice}

The results of the present study suggest that academic teacher education (in typical German universities) has little sustainable effect on teachers' beliefs about teaching and learning. Although universities are successful in the education of further teachers' generations, new and promising views of learning and teaching may still insufficiently reach the schools (Korthagen, 2010). There appears to be a divide between theory and practice as university studies emphasize academic knowledge whereas teaching at schools is based on teacher's craft knowledge (e.g., Broekkamp \& Van Hout-Wolters, 2007; Burkhardt \& Schoenfeld, 2003). When student teachers move from university to a new school they perceive that their beliefs, such as construction and participation, conflict with the existing school culture and priorities (Cooney, 1985; Raymond, 1997; Ruthven, 2002). The transition from the university into a school field, which may be full of discrepancies and intensified by emotional problems such as feelings of uncertainty, was often described in terms of "reality shock" (Dann et al., 1978). It should compel the beginning teachers to continuously transform their progressive beliefs toward a more traditional one (Veenman, 1984; Skott, 2002). In addition, the school and its like-minded teachers lead a beginning teacher to integrate into the new community. Additionally, the worst-case scenario may be the "corruption" of a new teacher by the institution school, according to the socialization theory (e.g., Hänsel, 1975).

Against this background, the results of the present study are consistent with the assumption that teacher education as well as bodies involved in the professional development of teachers should be aware of these circumstances. Teacher education should include the existing beliefs of student teachers in the context of education. Furthermore, professional development should also provide opportunities for practicing teachers to scrutinize their own beliefs about teaching and learning against the backdrop of innovative views congruent with the current research. Therefore, professional development constitutes an appropriate framework to establish the connection between the current research and school culture, thus contributing to the success of the teacher education. 


\subsection{Study limitation and implication for further research}

There are no studies which had compared the several stages of the teaching career. In that regard, the design of the present study could be seen as remarkable: There were five stages of a teaching career to compare: the beginning, middle and end of teacher education, the commencement of teaching in an actual school setting, and advanced teaching careers.

Despite the comprehensive results of the present study, there are also some limitations and open-ended questions that need to be addressed. One limitation refers to the content analysis of the interviews. The coding scheme to investigate the beliefs transmission, construction, participation and nurturing was developed on the basis of previous literature. This analysis produced useful results. Nevertheless, it should be acknowledged that this methodology was unable to identify new, as yet undescribed beliefs, which may have appeared during the teachers' self-reflections on learning and teaching. Further research, applying inductive method, is needed to evaluate the possible range of different views on learning and teaching.

Another limitation refers to the cross-sectional design of the study. The presented results may not be interpreted as a change in teachers' beliefs over their careers. Hence, there is a further need to extend the present research to a longitudinal study design. Obviously, the development of teachers' beliefs has important consequences for teacher education as well as professional development. For this reason, a longitudinal investigation of teachers' beliefs in broader teacher groups is indispensable.

The sample of the present study represents yet another limitation. The sample size was both insufficient and limited to one university. Although this university is responsible for the education of roughly 30 percent of all teachers for the given federal state of Germany, the applicability of the results should be considered carefully. Hence, there is a need for further research with a broader sample. 


\section{$5 \quad$ Konstruktion und Validierung des Fragebogens zur Messung der vier Lehrerüberzeugungen zum Lehren und Lernen (Studie 2)}

\subsection{Zusammenfassung und Abstract}

\section{Zusammenfassung}

Lehrerüberzeugungen zum Lehren und Lernen spielen eine wichtige Rolle in LehrLern-Prozessen. Die aktuelle Forschung hat in erster Linie knowledge transmission (dt. Transmission) und cognitive construction (dt. Konstruktion) untersucht, während andere Überzeugungen vernachlässigt wurden. Im Rahmen der vorliegenden Studie wurde ein Instrument zur Erfassung der vier Lehr-Lern-Überzeugungen - Transmission, Konstruktion, Partizipation und Schülerorientierung - entwickelt und validiert. Die faktorielle Struktur wurde an einer Stichprobe von $N=276$ Lehramtsstudierenden mittels einer exploratorischen Faktorenanalyse ermittelt. Reliabilitätsanalysen zeigten gute interne Konsistenzen der Skalen. Zusammenhangsanalysen mit konstruktnahen Variablen sowie Geschlechts- und Fächerkombinationsvergleiche wiesen auf die konvergente Konstruktvalidität des Fragebogens hin sowie auf seine Sensitivität gegenüber geschlechts- und fächerkombinationsspezifischen Unterschieden. Darüber hinaus gaben Unterschiede zwischen Bachelor- und Masterstudierenden Hinweise auf die Veränderung der Überzeugungen im Verlauf des Studiums.

\section{Abstract}

Teachers' beliefs about learning and teaching play a significant role in classroom activities. Recent research has primarily examined knowledge transmission and cognitive construction (Staub \& Stern, 2002), while other beliefs have been neglected. The present study aims to construct and validate a questionnaire in order to measure four different beliefs about learning and teaching: transmission, construction, participation and nurturing. Using a sample of $N=276$ student teachers, the factorial structure of the questionnaire was determined by means of exploratory factor analysis. Internal consistencies of the scales were determined to be sufficient. Correlations with variables closely related to the measured constructs, as well as gender- and domain-comparisons, show the convergent construct validity of the questionnaire. Its sensitivity regarding gender and domain specific differences in the beliefs about learning and teaching is sufficient as well. Furthermore, the differences between 
Kapitel 5

bachelor and master student teachers regarding their beliefs about learning and teaching indicate the change of the beliefs through the stages of their studies. 


\section{$5.2 \quad$ Einleitung}

Bereits während der Schulzeit, über das Studium hinweg und auch noch während ihrer Berufstätigkeit entwickeln Lehrkräfte Vorstellungen über das Lehren und Lernen. Diese Überzeugungen beeinflussen die Wahrnehmung der Lehrkräfte (Hashweh, 1996), ihre Interpretation des Unterrichtsgeschehens (Calderhead \& Robson, 1991), ihre Ziele (Aguirre \& Speer, 2000) und die an die Schülerinnen und Schüler gerichteten Erwartungen. Den Lehrerüberzeugungen wird eine handlungssteuernde Funktion zugesprochen (Brunner et al., 2006). Sie nehmen Einfluss auf das unterrichtliche Handeln der Lehrkräfte, und dies wirkt sich letztlich auf die Leistungen ihrer Schülerinnen und Schüler aus (z.B. Peterson et al., 1989; Staub \& Stern, 2002). Empirische Studien lieferten bereits Belege für diesen Zusammenhang. In der Studie von Staub und Stern (2002) mit 496 deutschen Grundschulkindern erzielten diejenigen Schülerinnen und Schüler bessere Mathematikleistungen, deren Lehrkräfte konstruktivistische Überzeugungen besaßen, im Vergleich zu Lehrkräften mit transmissiven Überzeugungen. Auch stellten die Lehrkräfte mit konstruktivistischen Überzeugungen anspruchsvollere Textaufgaben und boten somit mehr Lerngelegenheiten zum tieferen Konzeptverständnis an. Die Ergebnisse der COACTIV-Studie zeigten ebenfalls, dass der Unterricht von Lehrkräften mit konstruktivistischen Überzeugungen kognitiv aktivierend verlief und die Schülerinnen und Schüler konstruktive Unterstützung bekamen, was wiederum ihre Mathematikleistung förderte (Dubberke et al., 2008). Lehrerüberzeugungen zum Lehren und Lernen spielen somit eine wichtige Rolle für das schulische Lernen und stellen ein bedeutsames Konstrukt für die Lehrerforschung dar. Vor allem im Rahmen der Diskussion um Lehrerkompetenzen werden sie als eine Chance aufgefasst, das Lehrerhandeln besser erklären und gegebenenfalls verändern $\mathrm{zu}$ können. Daher dürfen die Überzeugungen angehender Lehrkräfte während der Lehrerausbildung nicht vernachlässigt werden, denn ohne eine Entwicklung der Überzeugungen bleibt das im Studium angeeignete Wissen träge (Renkl, 1996) und wird nicht genutzt. Die Aneignung fortschrittlicher Überzeugungen kann daher als Ziel der Lehrerausbildung betrachtet werden. Der erste Schritt liegt dabei in der Auseinandersetzung mit bestehenden Überzeugungen (Verloop et al., 2001), denn wenn die bereits vorhandenen Überzeugungen außer Acht gelassen werden, ist das Entwickeln von Überzeugungen nicht möglich (Trigwell, Prosser \& Taylor, 1994). Dies gilt genauso für den Bereich der beruflichen Weiterbildung, in der der erste Schritt idealerweise in einer Auseinandersetzung mit den eigenen vorhandenen Überzeugungen zum Lehren und Lernen besteht und somit gleichzeitig eine Methode zur 
Kapitel 5

Reflexion über das eigene Unterrichtshandeln und eine eventuelle Anpassung des Handelns darstellt. 


\subsection{Theoretischer Hintergrund}

\subsubsection{Lehrerüberzeugungen zum Lehren und Lernen: \\ Definition und Begriffsklärung der vier Überzeugungen}

Lehrerüberzeugungen zum Lehren und Lernen (engl. teachers 'beliefs about teaching and learning) sind subjektive und bewertende, auf Erfahrungen basierende Annahmen über das Lehren und das Rollenverständnis des Lehrerberufs sowie über das Lernen und die Aufgaben der Schülerinnen und Schüler. In der viel zitierten COACTIV-Studie werden Lehrerüberzeugungen nach Op’t Eynde, DeCorte und Verschaffel (2002) als implizite und explizite, subjektiv für wahr gehaltene Konzeptionen definiert. Sie sind affektiv und evaluativ (Nespor, 1987; Nisbett \& Ross, 1980) und werden als Filter der Wahrnehmung (Kagan, 1992; Pajares, 1992; Weinstein, 1990) und als Handlungsdisposition (Brown \& Cooney, 1982; siehe auch Bandura, 1986; Dewey, 1933; Nisbett \& Ross, 1980; Rokeach, 1968) verstanden.

In der Literatur wird oft zwischen den Überzeugungen Transmission und Konstruktion unterschieden (z.B. Baumert \& Kunter, 2008; Dubberke et al., 2008; Peterson et al., 1989; Staub \& Stern, 2001; Kunter, Klusmann, Dubberke, Baumert, Blum \& Brunner, 2007). Dabei wird Lernen nach der transmissiven Überzeugung als Übertragung von (strukturierten) Lerninhalten von der Lehrkraft zu den Schülerinnen und Schülern verstanden (Sfard, 1998) und nach der konstruktivistischen Lehr-Lern-Überzeugung als ein aktiver Prozess der eigenen Wissenskonstruktion des Schülers (z.B. Bereiter, 1994). Bei der Überzeugung Transmission liegt der Fokus auf Lerninhalten und Kenntnissen, die zum Eigentum eines Schülers werden, bei der Überzeugung Konstruktion verschiebt sich dieser Fokus vom Lehrer auf den Schüler mit seinem Vorwissen und seinen Interessen, die für den Lernprozess wichtig sind. Diese beiden Überzeugungen werden oft als Gegensätze dargestellt (z.B. Dubberke et al., 2008; Fennema et al., 1990; OECD; 2009; Olafson \& Schraw, 2006; Staub \& Stern, 2002).

Ein relativ geringer Anteil der Untersuchungen zu Lehr-Lern-Überzeugungen befasst sich neben diesen beiden auch mit anderen, wie zum Beispiel mit partizipatorischen und schülerorientierten Lehr-Lern-Überzeugungen. Der partizipatorischen Überzeugung nach wird das Lernen mit der Enkulturation eines neuen Mitglieds in die Lerngemeinschaft gleichgesetzt (vgl. legitimate peripheral participation nach Lave \& Wenger, 1991; learning communities nach Bielaczyc \& Collins, 1983; engaging in community nach Alger, 2009; participation metaphor nach Sfard, 1998) und das Lernziel im individuellen Wachstum mithilfe kollektiven

Wissensaufbaus gesehen. Die Überzeugung Schülerorientierung stellt die 
Schülerpersönlichkeit und die Lehrer-Schüler-Beziehung in den Mittelpunkt (vgl. Schülerorientierung nach Klieme et al., 2006; nurturing nach Alger, 2009; Pratt, 2005). Hier wird das Lernen als persönliches Wachstum und Entwicklung individuell angelegter Fähigkeiten in einer angemessenen Lernatmosphäre verstanden, welche durch ein warmes, unterstützendes Lehrerverhalten und eine individuelle Unterstützung des Schülers ermöglicht wird (Klieme et al., 2006).

Studien, die sich mit partizipatorischen und schülerorientierten Überzeugungen befassen, verwendeten die sowohl in der Durchführung als auch in der Auswertung aufwändige Interviewmethode, sodass die Vergleichbarkeit der Ergebnisse solcher Studien nicht gewährleistet wurde und keine größeren Stichproben untersucht werden konnten. Zudem wurden interessante Fragen, zum Beispiel nach dem Einfluss dieser Überzeugungen auf das unterrichtliche Handeln der Lehrkräfte oder auf das Schülerlernen nicht bearbeitet. Außerdem konzentrierte sich die bisherige empirische Forschung, die sich quantitativer Methoden bediente, auf die Überzeugungen Transmission und Konstruktion, sodass andere Überzeugungen vernachlässigt wurden (z.B. Dubberke et al., 2008; Fennema et al., 1990; OECD; 2009; Olafson \& Schraw, 2006; Staub \& Stern, 2002). Die vorliegende Studie geht gezielt über die bisherigen Ansätze in der Forschung zu Lehr-Lern-Überzeugungen hinaus, indem sie Partizipation und Schülerorientierung untersucht. Diese beiden Überzeugungen werden auf der Grundlage theoretischer Lernansätze sowie bisheriger Studien definiert. Im nächsten Kapitel werden existierende Instrumente zur Erfassung von Lehrerüberzeugungen zum Lehren und Lernen dargestellt und der Bedarf eines Instruments zur Erfassung besonders der beiden bislang wenig untersuchten Überzeugungen Partizipation und Schülerorientierung abgeleitet.

\subsubsection{Stand der Forschung zur Erfassung von Überzeugungen zum Lehren und Lernen}

Da sich die bisherige empirische Forschung auf Transmission und Konstruktion konzentrierte (z.B. Peterson et al., 1989; Staub \& Stern, 2002; Kunter et al., 2007), existieren einige Messinstrumente zur Erfassung dieser zwei Überzeugungen. Zum Beispiel wurden in der aktuellen internationalen Studie Teaching and Learning International Survey (TALIS) der OECD die Überzeugung Transmission (engl. direct transmission belief) und Konstruktion (engl. constructivist belief) mit jeweils vier Items erhoben (Beispielitem für Transmission: „Effective/good teachers demonstrate the correct way to solve a problem”, für Konstruktion: „My role as a teacher is to facilitate students' own inquiry”) (OECD, 2009). 
Die meisten der aktuellen Studien, wie zum Beispiel die Studie von Staub und Stern (2002), orientierten sich an dem von Peterson et al. (1989) entwickelten Fragebogen, mit dessen Hilfe konstruktivistische Überzeugungen im Fach Mathematik mit fünf Subskalen und 48 Items erhoben wurden (Staub \& Stern, 2002). Ein Beispiel für ein positives Item der Skala „die Rolle des Schülers“ ist „Schüler lernen Mathematik am besten, indem sie selbst Wege zur Lösung von einfachen Textaufgaben entdecken“, ein Beispiel für ein negatives Item lautet „Schüler lernen Mathematik am besten, indem sie den Erklärungen der Lehrerin oder des Lehrers folgen“. Ein Beispiel für ein positives Item der Skala „die Rolle des Lehrer“ lautet: „Man sollte Schülern erlauben, sich eigene Wege zur Lösung von einfachen Textaufgaben auszudenken, bevor die Lehrperson vorführt, wie diese zu lösen sind“" und ein Beispiel für ein negatives Item: „Am besten lehrt man Schülern das Lösen von Textaufgaben, indem man nicht verschiedene Arten von Textaufgaben zugleich behandelt, sondern sich jeweils auf eine Art beschränkt". Somit wird hier eine Bipolarität der beiden Überzeugungen angenommen: Die Zustimmung zu den negativen Items bedeutet niedrigere Werte für die Gesamtskala konstruktivistischer Überzeugungen. Damit werden Transmission und Konstruktion als zwei Pole auf einem Kontinuum des eindimensionalen Konstrukts der Überzeugungen verstanden.

Im deutschsprachigen Instrument der COACTIV-Studien wurden transmissive und konstruktivistische Überzeugungen mit mehreren Skalen erhoben: zum Beispiel mit der Skala „epistemologische Überzeugungen“, der Skala „Überzeugungen über das Mathematiklernen“ und der Skala „Zielvorstellungen zum Unterricht“ (Baumert et al., 2008). Ein Beispielitem für die konstruktivistische Überzeugung lautet: „Mathematik unterrichten heißt, Schüler/innen zum Nachdenken über mathematische Zusammenhänge zu bringen“. Ein Beispielitem für die transmissive Überzeugung ist: „Um im Mathematikunterricht erfolgreich zu sein, muss man Regeln und Verfahren sicher beherrschen.“ Durch die verschiedenen Skalen liegt die Annahme der Mehrdimensionalität von Überzeugungen vor; die Überzeugungen Transmission und Konstruktion stellen also distinktive Überzeugungen dar oder lassen sich zumindest voneinander abgrenzen.

In der PaLea-Studie zur Entwicklung berufsbezogener Merkmale von Studierenden im Verlauf des Lehramtsstudiums wurden unter anderem subjektive Lern-/Lehrtheorien bestehend aus 18 Lern- und 13 Lehrbegriffen erfasst (Bauer et al., 2010). Die Studierenden wurden gebeten, auf einer Skala von 1 - „,nicht ähnlich“ bis 4 - ,sehr ähnlich“ anzugeben, inwieweit der jeweilige Begriff ihrer Vorstellung von Lernen bzw. Lehren entspricht. Die Lernbegriffsskala bestand aus drei Subskalen: „Pauken“ (Beispielitem: „Lernen ist, sich anstrengen“), „Explorieren“ (Beispielitem: „Lernen ist, sich entwickeln“) und „Verstehen“ 
(Beispielitem: „Lernen ist begreifen“). Die Lehrbegriffsskala umfasste zwei Subskalen: „instruktionsorientiert“ (Beispielitem: „Lehren ist kontrollieren“) und „lernbegleitungsorientiert“ (Beispielitem: „Lehren ist Interesse wecken“).

Die hier genannten Instrumente zu Lehr-Lern-Überzeugungen beziehen sich auf Transmission und Konstruktion, partizipatorische und schülerorientierte Überzeugungen wurden bislang weniger untersucht. Darüber hinaus existieren keine validen Erhebungsinstrumente für Lehr-Lern-Überzeugungen, die die Idee des in der Literatur prominenten Lerngemeinschaftsansatzes (Lave \& Wenger, 1991) oder die Idee des Konstrukts der Schülerorientierung (Klieme et al., 2006; Pratt, 2005) berücksichtigen. Gleichwohl wurden ihre Inhalte in Studien dargestellt, die die Lehr-Lern-Überzeugungen angehender Lehrkräfte beschreiben (Brookhart \& Freeman, 1992; Wan Ng et al., 2010; Rathbone \& Pierce, 1990; Weinstein, 1989; Wideen et al., 1998). Zudem wurden sie in qualitativen Studien untersucht (z.B. Alger, 2009; Martínez et al., 2001) und auch mithilfe des Instruments „Teaching Perspective Inventory“ (TPI) nach Pratt (2005) erhoben. Neben der Skala zur Transmission (Beispielitem: „My interest is to prepare people for examinations") und der Skala zur Konstruktion (Beispielitem: „Teaching should build upon what people already know") beinhaltet das TPI Skalen zur Schülerorientierung (engl. nurturing) (Beispielitem: „My interest is to build people's self-confidence and self-esteem as learners”) sowie zu Apprenticeship (Beispielitem: „The best learning comes from working alongside good practitioners"). Während dieses Instrument recht umfassend von den Autoren eingesetzt wurde, wurden keine Validierungsstudien publiziert und auch die Validierung einer übersetzten deutschsprachigen Version durch eine Freiburger Arbeitsgruppe führte bisher nicht zu befriedigenden Ergebnissen (vgl. Hanke, 2011). Bereits die Items ließen sich nicht den theoretischen Dimensionen zuordnen, sodass die Faktorenstruktur nicht repliziert werden konnte. Offensichtlich ist es nicht zielführend, die Items des TPI möglichst eng am englischen Original ins Deutsche zu übersetzen.

Aus diesem Grund wurde als Ziel der vorliegenden Arbeit die Entwicklung eines Instruments zur Erfassung verschiedener Lehr-Lern-Überzeugungen definiert. Der neu entwickelte „Fragebogen zur Messung der vier Lehrerüberzeugungen“ (engl. The Questionnaire of Four Teachers' Beliefs - Q4TB) Transmission, Konstruktion, Partizipation und Schülerorientierung soll im Rahmen der vorliegenden Untersuchung zum Zweck seiner Validierung eigesetzt werden. Die im Vorfeld der Fragebogenentwicklung durchgeführten Interviews mit (angehenden) Lehrkräften konnten aufzeigen, dass sowohl partizipatorische als auch schülerorientierte Lehr-Lern-Überzeugungen in den Ansichten angehender und 
erfahrener Lehrkräfte präsent sind. Ausgehend von diesen Ergebnissen konnte mit der Entwicklung des Q4TB das Ziel verfolgt werden, ein Instrument zu entwickeln, welches gezielt über die bisherigen Ansätze in der Forschung zu Lehr-Lern-Überzeugungen von Lehrkräften hinausgeht, indem es zusätzlich zu den Überzeugungen Transmission und Konstruktion die Überzeugungen Partizipation und Schülerorientierung valide und reliabel misst. Mit diesem neu entwickelten Fragebogen soll die Erhebung der bei Lehrkräften bereits existierenden und sich während der Lehrerausbildung und Lehrerlaufbahn verändernden LehrLern-Überzeugungen ermöglicht werden. Die Erkenntnisse über diese Überzeugungen und ihre Entwicklung werden als gewinnbringend für die Lehrerforschung und die Lehrerbildung erachtet.

\subsection{3 Überblick über die Forschung zur Veränderung der Überzeugungen zum Lehren und Lernen}

In der Literatur wird immer wieder die Änderungsresistenz der Lehrerüberzeugungen betont (Kagan, 1992; Pajares, 1992; Weinstein, 1990). Dennoch erscheint eine Entwicklung der Überzeugungen im Verlauf der Lehrerlaufbahn plausibel, denn aufgrund erworbener Kenntnisse in der Lehreraus- und -weiterbildung und aufgrund zahlreicher Erfahrungen aus Lehr-Lern-Kontexten stehen Lehrerüberzeugungen unter vielfältigem Einfluss. Da Lehrerüberzeugungen gemachte Erfahrungen repräsentieren (z.B. Sigel, 1985), ist deren Veränderung während der Lehrerlaufbahn zu erwarten. Allerdings besteht ein Mangel an Untersuchungen, die die Entwicklung der Lehr-Lern-Überzeugungen demonstrieren. So beschränken sich viele Studien auf eine Beschreibung der Überzeugungen von Studierenden am Anfang ihrer Lehrerausbildung (z.B. Calderhead, 1996; Wan Ng et al., 2010; Russel, 1988; Richardson, 1996). Dabei wurden diese als emotionsorientiert und transmissiv dargestellt. Vereinzelt wurd berichtet, dass sich Lehr-Lern-Überzeugungen im Verlauf des Studiums von einer Emotions- zu einer Aufgabenorientierung (Nettle, 1998), von SchülernHelfen zu Klassen-Managen (Rust, 1994) verändern. Diese Darstellung spiegelt Inhalte der beiden Überzeugungen wider: die Emotionsoreintierung und das Schülern-Helfen spiegeln die Überzeugung Schülerorientierung wider, sowie die Aufgabenorientierung und das KlassenManagen die Überzeugung Transmission. Die beschriebene Entwicklung wurde in der ersten Studie (Interview-Studie mit angehenden und erfahrenen Lehrkräften) der vorliegenden Arbeit ähnlich vorgefunden: Die Überzeugung Schülerorientierung, die am Anfang des Studiums noch wichtig war, verlor bereits in der Mitte des Studiums ihre Bedeutung und auch 
am Ende des Studiums lagen die Werte hinsichtlich der Schülerorientierung signifikant niedriger als am Anfang des Studiums (Studie 1: Schlichter, Nückles \& Watermann, in Vorbereitung). Auch andere Studien mit querschnittlichen Vergleichen angehender Studierender mit solchen, die sich am Ende ihrer Lehrerausbildung befanden, lieferten Hinweise auf eine Veränderung der Überzeugungen (Saban, 2010; Saban et al., 2007). Die aktuelle Studie von Leavy et al. (2007) zeigte im Einklang mit den Ergebnissen der früheren Studie von Hollingsworth (1989), dass sich die Überzeugungen zukünftiger Lehrkräfte im Verlauf der Lehrerausbildung in Richtung Konstruktivismus veränderten. Dies ist auch das Ergebnis der Interviewstudie der vorliegenden Arbeit (Studie 1: Schlichter et al., in Vorbereitung): Im Verlauf des Studiums gaben Lehramtsstudierende ihre Präferenz für die Schülerorientierung zugunsten konstruktivistischer Überzeugungen auf.

Die hier dargestellten Studien stellen querschnittliche Vergleiche dar. Auch wenn die Interviewstudie der vorliegenden Arbeit drei Zeitpunkte in der Lehrerausbildung untersuchte, handelt es sich streng genommen nicht um die Entwicklung der Überzeugungen. Gleichwohl können die berichteten Ergebnisse als Hinweise darauf interpretiert werden, dass die Lehrerausbildung einen Einfluss auf die Entwicklung von Lehr-Lern-Überzeugungen ausübt. Insgesamt fällt in diesem Zusammenhang der Mangel an Untersuchungen zur partizipatorischen Überzeugung und ihrer Entwicklung besonders auf. Die Interviewstudie der vorliegenden Arbeit (Studie 1: Schlichter et al., in Vorbereitung) stellt den ersten Versuch in diese Richtung dar. Die Ergebnisse der Studie demonstrieren, dass sich auch Partizipation, ähnlich wie die anderen drei Überzeugungen, im Verlauf des Studium verändert: Am Anfang des Studiums zeigten Lehramtsstudierende niedrige Werte hinsichtlich dieser Überzeugungen, bereits in der Mitte stieg die Präferenz der Studierenden für Partizipation deutlich an und bis zum Ende des Studiums blieben die Werte für die partizipatorische Überzeugung auf einem hohen Niveau. Die Analyse dominierender Überzeugungen der Studierenden demonstrierte, dass am Anfang des Studiums die transmissiven und die schülerorientierten Überzeugungen am meisten bevorzugt wurden: Vier bzw. drei der zehn Studierenden am Anfang des Studiums gaben an, diese Überzeugungen als für sie dominierend zu empfinden. Während in der Mitte des Studiums die partizipatorischen und die konstruktivistischen Überzeugungen als dominierende Überzeugungen identifiziert wurden, zeigte nur die konstruktivistische Überzeugung am Ende des Studiums ihre Dominanz (drei von zehn Studierenden hatten diese als dominierende Überzeugung).

Es lässt sich festhalten, dass sich Lehr-Lern-Überzeugungen im Verlauf der Lehrerausbildung zu verändern scheinen bzw. dass in Abhängigkeit vom Zeitpunkt in der 
Lehrerausbildung unterschiedliche Überzeugungen präferiert werden. Die bisherige Forschung zur Frage nach der Veränderung der Lehr-Lern-Überzeugungen kann nicht als ausreichend bezeichnet werden. Daher wird im Folgenden unter anderem die Frage nach den Unterschieden zwischen Lehr-Lern-Überzeugungen in Abhängigkeit vom Zeitpunkt im Studium untersucht. Dafür werden die Überzeugungen von Bachelor- mit denen von Masterstudierenden miteinander verglichen.

\subsubsection{Epistemologische Überzeugungen und Lehr-Lern-Überzeugungen}

Epistemologische Überzeugungen werden als Ansichten über die Natur von Wissen, dessen Struktur und Genese definiert. Ähnlich wie Lehr-Lern-Überzeugungen strukturieren sie die Art der Begegnung mit der Welt vor - so die theoretische Annahme (Duell \& Schommer-Aikins, 2001; Hofer, 2000, 2001; Hofer \& Pintrich, 1997; Howard et al, 2000; Schommer, 1990, 1994b). Die Forschung zu epistemologischen Überzeugungen weist einige gut evaluierte Messinstrumente auf. Von den deutschsprachigen Instrumenten ist besonders das Instrument von Trautwein und Lüdtke (2004) zu erwähnen. Mit Hilfe dieses Fragebogens lassen sich domänenübergreifende epistemologische Überzeugungen mit einer relativ kurzen Skala erheben. Die 15 Items beziehen sich auf die Kerndimension „Struktur des Wissens“ mit den Subskalen zu Dualismus und Relativismus. Die Dualismusskala (fünf Items) thematisiert das Vertrauen in wissenschaftliche Erkenntnisse und in die Fähigkeit der Wissenschaft, eines Tages alles erklären zu können. Hohe Werte auf dieser Skala signalisieren ein unkritisches Verhältnis zu wissenschaftlichen Erkenntnissen. Eine hohe Ausprägung auf der Relativismusskala signalisiert, dass man kritisch mit wissenschaftlichen Theorien umgeht und dass einem bewusst ist, dass sie das Ergebnis menschlicher Konstruktionsleistungen darstellen.

Die Verbindung zwischen epistemologischen und lehr-/lernbezogenen Überzeugungen wurde in der einschlägigen Literatur diskutiert (Clark \& Peterson, 1986; Fennema \& Loef Franke, 1992; Marland, 1995; 1998; Richardson, 1996). Es wurde angenommen, dass eine bestimmte Vorstellung vom Wissen eine bestimmte Vorstellung vom Lernen mit sich bringt. Zum Beispiel sollen Lehrkräfte, die das Wissen als einfach und gesichert auffassen, eine rezeptive Überzeugung zum Lernen haben, und Lehrkräfte, die das Wissen als ein Netzwerk von Theorien verstehen, der Überzeugung sein, dass Schülerinnen und Schüler durch Verständigungs- und Aushandlungsprozesse ihr Wissen selbst konstruieren (Olafson \& Schraw, 2006). Die empirische Forschung bestätigte bereits diese Annahme: Seidel et al. 
(2008) konnten Zusammenhänge zwischen einem Wissensverständnis als objektive, von individuellen Sichtweisen unabhängige Realität (empiristisches Wissensverständnis) und einer transmissiven Überzeugung zum Lernen $(r=.28, p<.05)$ sowie zwischen einem Wissensverständnis als einem Netzwerk von sich ständig verändernden Theorien (konstruktivistisches Wissensverständnis) und einer konstruktivistischen Sicht auf Schülerlernen $(r=.41, p<.005)$ aufzeigen. Von ähnlichen Ergebnissen berichteten Chan und Elliott (2004). In ihrer Studie hingen die Lehr-Lern-Überzeugungen Transmission mit naiven epistemologischen Überzeugungen zusammen: dem Glauben an eine angeborene Lernfähigkeit $(r=.40, p<.001)$, an Expertenwissen $(r=.40, p<.001)$ und an sicheres Wissen $(r=.31, p<.001)$. Dagegen zeigte sich ein negativer Zusammenhang zwischen der Lehr-Lern-Vorstellung Konstruktion und den naiven Epistemologien: den angeborenen Lernfähigkeiten $(r=-.16, p<.01)$ sowie dem Expertenwissen $(r=-.22, p<.001)$, aber ein positiver Zusammenhang zwischen der konstruktivistischen Lehr-Lern-Vorstellung und der fortgeschrittenen epistemologischen Überzeugung - dem Glauben an Lernanstrengung $(r=$ $.39, p<.001)$.

\subsubsection{Geschlechtsspezifität der Überzeugungen zum Lehren und Lernen}

Die empirische Forschung lieferte Hinweise auf die Geschlechtsspezifität von Überzeugungen zum Lehren und Lernen. In der Untersuchung von Pratt et al. (2001) schienen Studentinnen im Vergleich zu Studenten die Perspektive Schülerorientierung und Konstruktion $\mathrm{zu}$ bevorzugen. Auch in der Studie von Norton et al. (2005) wiesen Studentinnen höhere Werte in der Überzeugung Konstruktion auf als ihre männlichen Kommilitonen, die nämlich höhere Werte in der Transmission zeigten.

\subsubsection{Fachspezifität der Überzeugungen zum Lehren und Lernen}

Ähnlich der Geschlechts- wird eine Fachspezifität der Überzeugungen zum Lehren und Lernen angenommen. Es gibt eine Reihe von Studien, die dadurch, dass sie ausschließlich im Bereich der Mathematik durchgeführt wurden, die Frage nach der Fachspezifität der Überzeugungen ausgeklammert haben, um etwaige Effekte auszuschließen (Peterson et al., 1989; Kunter et al., 2007; Staub \& Stern, 2002). Studien, die verschiedene Fächer miteinander vergleichen, liefern eine empirische Evidenz für Unterschiede in den Lehr-LernÜberzeugungen in Abhängigkeit vom Fach. So schienen Studierende der 
Geisteswissenschaften die Überzeugungen Konstruktion zu bevorzugen, während Studierende der Naturwissenschaften höhere Werte in der Transmission aufwiesen (Norton et al., 2005). Studierende der Sozialwissenschaften erzielten höhere Werte bezogen auf die Konstruktion und die der Mathematik und Naturwissenschaften bevorzugten die Transmission (Collins, Jarvis-Sellinger \& Pratt, 2001; Norton et al., 2005).

Zusammenfassend lässt sich festhalten, dass sich Lehrerüberzeugungen in Abhängigkeit vom Zeitpunkt der Lehrerausbildung, vom Geschlecht sowie vom Fach der (angehenden) Lehrkräfte zu unterscheiden scheinen und in einem bestimmten Verhältnis zu epistemologischen Überzeugungen stehen. Im Folgenden wird die Entwicklung eines Messinstruments dargestellt, dessen Validität mittels der Vergleiche der Lehr-LernÜberzeugungen in Abhängigkeit vom Zeitpunkt in der Lehrerausbildung, vom Geschlecht sowie vom studierten Fach angehender Lehrkräfte sowie mittels Zusammenhangsanalysen mit epistemologischen Überzeugungen eingeschätzt wird. 


\subsection{Ableitung der Fragestellung}

Zur Entwicklung des Q4TB wurde eine Studie mit Studierenden des Lehramtes einer deutschen Universität durchgeführt. Das Hauptanliegen bestand in einer ersten Validierung des neu entwickelten Fragebogens. Um Hinweise für die Faktorstruktur des Fragebogens zu gewinnen, wurden die mittels einer exploratorischen Faktorenanalyse erhaltenen Faktoren auf ihre Übereinstimmung mit den theoretisch formulierten Dimensionen der vier Überzeugungen überprüft.

Die erste Frage bezog sich demnach auf die faktorielle Struktur des Fragebogens: Lassen sich die vier Überzeugungen zum Lehren und Lernen und/oder deren Dimensionen mittels einer exploratorischen Faktorenanalyse bestätigen? Sind die gebildeten Skalen reliabel? Ausgehend von den Ergebnissen der Faktorenanalysen wurden Skalen entsprechend der theoretisch angenommenen Dimensionen gebildet. Zur Bestimmung der internen Konsistenz des Fragebogens wurden Reliabilitätskennwerte für die gebildeten Skalen bestimmt.

Die zweite Frage bezog sich auf die Konstruktvalidität des Fragebogens. Finden sich korrelative Zusammenhänge des Instruments Q4TB mit einem anderen Fragebogen zu subjektiven Lehr-/Lerntheorien (Skalen zu Lern-/Lehrbegriffen: Bauer et al., 2010) sowie zu epistemologischen Überzeugungen (Dualismus und Relativismusskalen: Trautwein \& Lüdtke, 2004)? Ausgehend von Ergebnissen bisheriger Forschung können Zusammenhänge der LehrLern-Überzeugung Transmission mit Skalen beider Messinstrumente erwartet werden, die ein naives epistemologisches Wissensverständnis wiedergeben. Für die Überzeugung Konstruktion lässt sich eine Verbindung mit einem Lernverständnis als Verstehen und einem Lehrverständnis als Lernbegleitung sowie dem relativistischen Wissensverständnis erwarten.

Der Zusammenhang zwischen epistemologischen Überzeugungen und den beiden anderen lehr-/lernbezogenen Überzeugungen wurde bisher nicht analysiert, sodass hier ausschließlich Vermutungen geäußert und keine Hinweise der bisherigen Forschung zu Rate gezogen werden können. Denkbar wäre ein Zusammenhang der Lehr-Lern-Überzeugung Partizipation, die den theoretischen Lerngemeinschaftsansatz (Lave \& Wenger, 1991) repräsentiert, mit einem verstehensorientierten Lernbegriff sowie einem lernbegleitungsorientierten Lehrverständnis. Da Partizipation das Lernen in einer Gemeinschaft mittels Diskussionen und Aushandlungsprozessen über gemeinsames Verständnis vom Wissen betont, kann ein Zusammenhang von Partizipation mit einer relativistischen epistemologischen Überzeugung erwartet werden. Zudem werden 
gemeinschaftliche Lernprozesse in einer Gruppe als ein wichtiger Aspekt des Lernens gesehen, somit können auch Zusammenhänge zu einigen sozialen Aspekten erwartet werden. Die Lehr-Lern-Überzeugung Schülerorientierung könnte möglicherweise Zusammenhänge mit dem lernbegleitungsorientierten Lehrbegriff aufweisen, da bei dieser Überzeugung der Schüler im Mittelpunkt des unterrichtlichen Geschehens steht.

Die dritte Frage zielte auf die Sensitivität des Fragebogens gegenüber geschlechts- und fachspezifischen Unterschieden von Überzeugungen ab. Die Fragestellung lautete: Lassen sich Unterschiede zwischen den mit dem Fragebogen erhobenen Werten in den Überzeugungen von Studentinnen und den Überzeugungen von ihren männlichen Kommilitonen sowie Unterschiede zwischen den Überzeugungen von Studierenden mit unterschiedlichen Fächerkombinationen ermitteln? Dazu wurden Studentinnen mit ihren männlichen Kommilitonen sowie Studierende mit unterschiedlichen Fächerkombinationen verglichen.

Die vierte Frage bezog sich auf eine mögliche Entwicklung in den Überzeugungen in Abhängigkeit vom Zeitpunkt im Studium. Verändern sich die Überzeugungen im Verlauf des Studiums? Der Quasi-Längsschnitt erlaubt den Vergleich der Überzeugungen von Bachelorund Masterstudierenden. Wie bereits erwähnt, wird davon ausgegangen, dass sich Lehr-LernÜberzeugungen mit den Jahren an Erfahrungen, aber auch schon in der Lehrerausbildung verändern. Daher können unter anderem aufgrund der im Studium vermittelten Kenntnisse der Lehr-/Lerntheorien, wie zum Beispiel dem Konstruktivismus, Veränderungen der Überzeugungen in die entsprechende Richtung erwartet werden.

Im Folgenden sollen das Instrument und die Ergebnisse der ersten Validierungsstudie vorgestellt werden. 


\subsection{Methode}

\subsubsection{Skalenkonstruktion des Fragebogens zur Messung der vier Lehrerüberzeugungen zum} Lehren und Lernen (engl. The Questionnaire of Four Teachers' Beliefs - Q4TB)

Der Fragebogen zur Erfassung der vier Überzeugungen zum Lehren und Lernen Transmission, Konstruktion, Partizipation und Schülerorientierung - wurde auf der Grundlage lern-/lehrtheoretischer Ansätze, existierender Instrumente sowie mit Hilfe der Ergebnisse der im Vorfeld durchgeführten Lehrerinterviewstudie entwickelt (vgl. Kap. 4).

Die empirische Forschung kann bereits einige Instrumente zu den transmissiven und konstruktivistischen Überzeugungen vorweisen. Daher wurden die Skalen des Q4TB zur Transmission und Konstruktion in Anlehnung an existierende Instrumente der COACTIVStudien (Baumert et al., 2008) und der BIJU-Studie (Bildungskarrieren im Jugend- und frühen Erwachsenenalter) formuliert (Bauer et al., 2010; Daniels, 2008).

Für die Überzeugung Partizipation gab es bisher kein Messinstrument. Hier wurde zur Orientierung der theoretische Lerngemeinschaftsansatz verwendet (vgl. legitimate peripheral participation nach Lave \& Wenger, 1991; learning communities nach Bielaczyc \& Collins, 1983; participation metaphor nach Sfard, 1998). Bei der Schülerorientierung wurde das theoretische Konstrukt der Schülerorientierung nach Klieme et al. (2006) mit den Aspekten „Autonomieunterstützung“ und „soziale Eingebundenheit“ genutzt. Zusätzlich wurden einige Items der Skalen aus der BIJU-Studie (Bauer et al., 2010; Daniels, 2008) für die Items zur Schülerorientierung adaptiert.

Um Items zu partizipatorischen und schülerorientierten Vorstellungen zu formulieren, wurde eine im Vorfeld der Fragebogenentwicklung durchgeführte Interviewstudie hinzugezogen. Diese Interviewstudie sollte in einem ersten Schritt prüfen, inwieweit die vier Überzeugungen in den Vorstellungen der Lehrkräfte zum Lehren und Lernen präsent sind. Die inhaltsanalytische Auswertung der Interviews bestätigte die subjektive Relevanz der vier Überzeugungen für die Lehrkräfte (vgl. Kap. 4). Auf die Frage danach, wie sie sich Lehren und Lernen vorstellen, wurden die Grundideen der vier Überzeugungen unterschiedlich oft angesprochen und unterschiedlich detailliert beschrieben. Zum Beispiel wurden zur Überzeugung Partizipation die Kategorien „Gemeinschaftliches Lernen unterstützen“ oder „Kommunikation“ inhaltsanalytisch gebildet. Typische Äußerungen solcher Facetten wurden als Ankerbeispiele extrahiert: „Ich versuche das gemeinschaftliche Lernen, das Wir-Gefühl, unter den Schülern zu fördern“, „Bei mir in der Klasse sollen sich die Schüler über den 
Unterrichtsstoff austauschen. Ich lasse sie sich gegenseitig Sachen erklären und erläutern“. Diese Ankerbeispiele wurden für die Formulierung der Items im neuen Fragebogen Q4TB genutzt: So sind auf der Grundlage der Ankerbeispiele Items entstanden: „.. das gemeinschaftliche Lernen unter den Schülerinnen und Schülern zu fördern“, „Das Wir-Gefühl in der Klasse zu fördern“, „... dass sich Schülerinnen und Schüler über die Lerninhalte austauschen“, „... die Schülerinnen und Schüler gegenseitig Sachverhalte erklären und erläutern zu lassen“.

In Tabelle 6 sind die Facetten der Überzeugungen aufgelistet sowie die Quelle der einzelnen Facetten angegeben. Es sind ebenfalls Beispielitems und die Anzahl der Items pro Facette aufgeführt. 


\section{Tabelle 6}

Facetten der vier Überzeugungen, die Quelle der Facetten, Beispielitems sowie Anzahl der verwendeten Items $(n)$

\begin{tabular}{llll}
\hline $\begin{array}{l}\text { Überzeugung: } \\
\text { Facette }\end{array}$ & Quelle & $\begin{array}{l}\text { Beispielitem: Unterrichten bedeutet } \\
\text { für mich... }\end{array}$ \\
\hline Transmission: Effektives & Eigenkonstruktion & $\begin{array}{l}\text {.. Lerninhalte zu präsentieren, } \\
\text { sodass sie von den Schüler/innen }\end{array}$ & 5 \\
Präsentieren & & $\begin{array}{l}\text { gut aufgenommen werden können. } \\
\text { gut }\end{array}$
\end{tabular}

Transmission: Vorführen, „Rezeptives Lernen Zeigen, Demonstrieren durch Beispiele und Vormachen“ (COACTIV, 2009)
... den Schülerinnen und Schülern 4 demonstrieren, wie man vorzugehen hat.

\begin{tabular}{|c|c|c|}
\hline $\begin{array}{l}\text { Transmission: } \\
\text { Wiederholen, } \\
\text { Zusammenfassen, } \\
\text { Überprüfen }\end{array}$ & $\begin{array}{l}\text { „Klarheit und } \\
\text { Strukturiertheit des } \\
\text { Unterrichts“" } \\
\text { (BIJU, 2008) }\end{array}$ & $\begin{array}{l}\text {... Lerninhalte regelmäßig zu } \\
\text { wiederholen, damit die } \\
\text { Schülerinnen und Schüler sich } \\
\text { diese gut einprägen können. } \\
\text {... den Unterrichtsstoff regelmäßig } \\
\text { zusammenzufassen, damit die } \\
\text { Schülerinnen und Schüler sich } \\
\text { diesen gut merken können. }\end{array}$ \\
\hline $\begin{array}{l}\text { Konstruktion: } \\
\text { Fehlerakzeptanz }\end{array}$ & $\begin{array}{l}\text { „Sokratisches Vorgehen“ } \\
\text { (BIJU, 2008) }\end{array}$ & $\begin{array}{l}\text {... auch Fehler der Schülerinnen } \\
\text { und Schüler zu akzeptieren und sie } \\
\text { weitermachen zu lassen, bis sie } \\
\text { selbst gemerkt haben, dass etwas } \\
\text { nicht stimmt. }\end{array}$ \\
\hline $\begin{array}{l}\text { Konstruktion: } \\
\text { Selbständiges und } \\
\text { Selbstbestimmtes Lernen }\end{array}$ & $\begin{array}{l}\text { „Selbstständiges und } \\
\text { verständnisvolles } \\
\text { diskursives Lernen“ } \\
\text { (COACTIV, 2009) }\end{array}$ & $\begin{array}{l}\text {.. die Schüler/innen mit ihren } \\
\text { eigenen Vermutungen auch eigene } \\
\text { Lernwege gehen zu lassen. }\end{array}$ \\
\hline $\begin{array}{l}\text { Partizipation: Teilhabe an } \\
\text { einer Lerngemeinschaft }\end{array}$ & Eigenkonstruktion & $\begin{array}{l}\text {.. zum Aufbau einer } \\
\text { Lerngemeinschaft beizutragen }\end{array}$ \\
\hline
\end{tabular}

Partizipation:

Wertschätzung in der

Lerngemeinschaft
Eigenkonstruktion

... die soziale Wertschätzung in der 4 Gruppe der Lernenden zu unterstützen.
Partizipation: Lehrer in der Lerngemeinschaft
Eigenkonstruktion
... als Lehrkraft selbst ein Teil der

4 


\begin{tabular}{|c|c|c|c|}
\hline $\begin{array}{l}\text { Überzeugung: } \\
\text { Facette }\end{array}$ & Quelle & $\begin{array}{l}\text { Beispielitem: Unterrichten bedeutet } \\
\text { für mich... }\end{array}$ & $\mathrm{n}$ \\
\hline $\begin{array}{l}\text { Partizipation: } \\
\text { Gemeinschaftliches } \\
\text { Lernen unterstützen }\end{array}$ & Eigenkonstruktion & $\begin{array}{l}\text {... dass die Schüler/innen sich } \\
\text { gegenseitig beim Lernen } \\
\text { unterstützen. }\end{array}$ & 3 \\
\hline $\begin{array}{l}\text { Partizipation: } \\
\text { Gemeinschaftlich } \\
\text { geteiltes Wissen }\end{array}$ & Eigenkonstruktion & $\begin{array}{l}\ldots \text { in einer Lerngemeinschaft das } \\
\text { Wissen zu teilen. }\end{array}$ & 3 \\
\hline $\begin{array}{l}\text { Partizipation: } \\
\text { Kommunikation }\end{array}$ & Eigenkonstruktion & $\begin{array}{l}\text {... die Schülerinnen und Schüler } \\
\text { über die Lerninhalte miteinander } \\
\text { diskutieren zu lassen. }\end{array}$ & 6 \\
\hline $\begin{array}{l}\text { Schülerorientierung: } \\
\text { Schülerautonomie }\end{array}$ & $\begin{array}{l}\text { „Schüler- } \\
\text { mitbestimmung““ } \\
\text { (BIJU, 2008) }\end{array}$ & $\begin{array}{l}\text {.. Schülerinnen und Schülern } \\
\text { Stoffe und Themen zur Auswahl zu } \\
\text { geben. }\end{array}$ & 4 \\
\hline $\begin{array}{l}\text { Schülerorientierung: } \\
\text { Soziale Eingebundenheit }\end{array}$ & $\begin{array}{l}\text { "Sozialorientierung der } \\
\text { Lehrkraft" } \\
\text { (BIJU, 2008) }\end{array}$ & $\begin{array}{l}\text {...sich auch Zeit für Schülerinnen } \\
\text { und Schülern zu nehmen, wenn sie } \\
\text { etwas bereden wollen. }\end{array}$ & 5 \\
\hline $\begin{array}{l}\text { Schülerorientierung: } \\
\text { Aufbau einer Beziehung } \\
\text { zu Schülerinnen und } \\
\text { Schülern }\end{array}$ & Eigenkonstruktion & $\begin{array}{l}\text {... Schülerinnen und Schülern } \\
\text { besser kennen zu lernen, um ihre } \\
\text { Lernergebnisse in den Kontext } \\
\text { ihrer Lebenssituation einordnen } \\
\text { und dadurch besser verstehen zu } \\
\text { können. }\end{array}$ & 6 \\
\hline
\end{tabular}

Insgesamt wurden 65 Items generiert, die die verschiedenen Facetten der vier Überzeugungen thematisieren. Der Itemstamm lautete für alle Items „Unterrichten bedeutet für mich..." und war in Fettdruck oben auf jeder Seite des Fragebogens platziert. Die einzelnen Items waren auf einer fünfstufigen Skala $(1=$ trifft nicht zu, $2=$ trifft eher nicht zu, $3=$ teils teils, $4=$ trifft eher $\mathrm{zu}, 5=$ trifft $\mathrm{zu}$ ) zu beantworten.

\subsubsection{Stichprobe und Design}

Insgesamt nahmen an der Untersuchung $N=276$ Lehramtsstudierende einer deutschen Universität teil, davon waren 63 Männer und 187 Frauen (bei 26 Personen fehlte die Geschlechtsangabe). Die 224 Studierenden befanden sich zum Zeitpunkt der Befragung am Anfang ihres Bachelorstudiums, 52 hatten ihr Bachelorstudium bereits abgeschlossen und absolvierten ihr Masterstudium. Die Bachelorstudierenden waren im Mittel $21.34(S D=1.62)$ und die Masterstudierenden $25.02(S D=1.57)$ Jahre alt. Die Befragten studierten 
naturwissenschaftliche Fächer wie Biologie, Mathematik oder Chemie, geisteswissenschaftliche Fächer wie Geschichte, Theologie oder Englisch. Da jeweils zwei Fächer studiert werden, ergaben sich folgende Kombinationen: Studierende mit beiden naturwissenschaftlichen Fächern $(n=15)$, mit beiden geisteswissenschaftlichen Fächern $(n=$ 119) und Studierenden mit einem naturwissenschaftlichen und einem geisteswissenschaftlichen Fach $(n=48)$. Die Erhebung bei den Bachelorstudierenden fand im Anschluss an eine Einführungsveranstaltung im ersten Semester statt. Die Befragung bei den Masterstudierenden erfolgte im Rahmen einer Vorlesung in den Bildungswissenschaften, die normalerweise im dritten Semester des Masterstudiums besucht wird.

\subsubsection{Instrumente}

Wertehaltungen und Überzeugungen: Lern- und Lehrbegriffsskalen, Erziehungsziele

Lehrerüberzeugungen zum Lehren und Lernen weisen eine inhaltliche Konstrukt-Nähe zu den Skalen subjektiver Lehr-/Lerntheorien der PaLea-Studie auf (Bauer et al., 2009; 2010). In der vorliegenden Studie wurden einige Skalen dieser Studie eingesetzt: die Lern- (18 Items) und die Lehrbegriffsskala (13 Items) sowie eine Skala zu Erziehungszielen (11 Items). Die Studierenden wurden gefragt, inwieweit der jeweilige Begriff ihrer Vorstellung von Lernen und Lehren entspricht bzw. als wie wichtig sie das jeweilige Erziehungsziel für die Schule erachten. Die Lernbegriffsskala bestand aus drei Subskalen „Pauken“ Beispielitem: „... sich anstrengen“), „Explorieren“ (Beispielitem: „... sich entwickeln“) und „Verstehen“ (Beispielitem: „.. begreifen“). Die Lehrbegriffsskala umfasste zwei Subskalen: „,instruktionsorientiert“ (Beispielitem: „... kontrollieren“) und „lernbegleitungsorientiert“ (Beispielitem: „... Interesse wecken“). Bei den „Erziehungszielen“ wurden zum Beispiel „Leistungs- und Anstrengungsbereitschaft“, „,vielseitiges Wissen“ oder „persönliche Selbstständigkeit“" zum Einschätzen hinsichtlich ihrer Wichtigkeit präsentiert. Die einzelnen Items waren auf einer vierstufigen Skala (von $1=$ nicht ähnlich/weniger wichtig bis $4=$ sehr ähnlich/äußerst wichtig) zu beantworten. Alle Skalen wiesen in der vorliegenden Untersuchung hohe interne Konsistenzen auf („Lernbegriff“"-Subskalen: „Pauken“ $\alpha=.83$; „Explorieren“ $\alpha=.66 ;$,,Verstehen“ $\alpha=.81$; ,Lehrbegriff“-Skalen: ,,instruktionsorientiert“ $\alpha=$ .74 ; ,lernbegleitungsorientiert“ $\alpha=.79$ ). 


\section{Epistemologische Überzeugungen}

Zur Erfassung allgemeiner epistemologischer Überzeugungen wurde ein Instrument (zehn Items) aus der TOSCA-Studie eingesetzt, das nach Meinung der Autoren die Kerndimension epistemologischer Überzeugungen „Struktur des Wissens“ erfasst (Trautwein \& Lüdtke, 2004). Die Skala umfasst drei Subskalen: (1) „Dualismus“ (vier Items) (Beispielitem „Wissenschaftliche Erkenntnis ist über jeden Zweifel erhaben“), wobei hohe Werte auf dieser Skala ein unkritisches Verhältnis zu wissenschaftlichen Erkenntnissen signalisieren; (2) „Relativismus“ (vier Items) (Beispielitem „Wissenschaftliche Theorien können sich auch jederzeit als falsch erweisen“), wobei hohe Werte das Bewusstsein dafür wiedergeben, dass wissenschaftliche Theorien menschliche Konstruktionen darstellen, sowie (3) „Wahrheitsgläubigkeit“ (zwei Items) (Beispielitem „Das Schöne an der Wissenschaft ist, dass von zwei Meinungen zu einem Thema sich eine schließlich als falsch erweist"). Für die Beantwortung stand den Studierenden eine vierstufige Skala (von $1=$ trifft überhaupt nicht zu bis 4 = trifft völlig zu) zur Verfügung. Die Reliabilitäten der Skalen liegen in der vorliegenden Untersuchung im zufriedenstellenden Bereich („Dualismus“: $\alpha=.63$; „Relativismus“: $\alpha=.63$; „Wahrheitsgläubigkeit": $\alpha=.54$ ).

\subsubsection{Umgang mit fehlenden Werten}

Bei der Analyse fehlender Werte wurden keine spezifischen Muster oder signifikanten Gruppenunterschiede im Ausmaß fehlender Werte festgestellt. Somit wurden keine Hinweise auf ein systematisches Auftreten fehlender Werte gefunden. Aufgrund dessen wurden in den Korrelations- und in den Faktorenanalysen die fehlenden Werte mit der Methode des paarweisen Fallausschlusses (pairwise deletion) behandelt (Bortz, 2005). 


\subsection{Ergebnisse}

\subsubsection{Faktorenstruktur}

\section{Faktorlösung ohne Vorgabe der Faktorenanzahl}

Zur Prüfung der Faktorenstruktur des Fragebogens wurde eine exploratorische Faktorenanalyse mittels der Hauptkomponenten-Analyse (PCA) mit Varimax-Rotation berechnet (vgl. Rost, 2007). Die Faktorenanalyse ergab 18 Eigenwerte über 1.0. Die Ergebnisse des Scree-Tests legten alternativ eine 4-Faktoren-Lösung nahe. In einem ersten Schritt wurde die Lösung mit den 18 Faktoren betrachtet. Die Items wurden zuerst auf heterogene Faktorladungen überprüft. So wurden zwei Items mit Nebenladungen über .40 aus der Analyse ausgeschlossen. Eine hohe und damit eindeutig zu einem Faktor zuordenbare Faktorladung wurde bei .45 angesetzt (fünf Items wurden aufgrund ihrer geringen Faktorladungen ausgeschlossen). Außerdem wurden die Faktoren nicht berücksichtigt, die nur ein Item mit einer definiert hohen Ladung aufwiesen. So wurden vier Items ausgeschlossen bzw. vier Faktoren nicht berücksichtigt. Insgesamt wurden bei diesem Vorgehen elf Items ausgeschlossen. Anschließend wurde die Faktorenanalyse mit den 54 verbliebenen Items wiederholt. Nach dem Kaiser-Kriterium resultierten 14 Faktoren mit einer Varianzaufklärung von 70.6 Prozent. Auch in dieser Analyse legte die Inspektion des Scree-Tests alternativ eine 4-Faktoren-Lösung nahe. Zunächst wurden jedoch 14 Faktoren extrahiert und diese Lösung betrachtet, um festzustellen, ob und in welchem Maße die 14 Facetten lehr-/lernbezogener Überzeugungen durch die identifizierten 14 Faktoren abgebildet werden. Die Kommunalitätenschätzungen konvergierten in 25 Iterationen. Mit wenigen Ausnahmen luden alle Items theoriekonform auf dem entsprechenden Faktor.

Auf einem Faktor luden alle sechs Items der theoretisch postulierten Dimension „Aufbau einer Beziehung zu Schülerinnen und Schülern“, somit konnte diese theoretisch postulierte Facette der Überzeugung Schülerorientierung faktorenanalytisch abgebildet werden. Ein Item wurde aufgrund der geringen Faktorladung ausgeschlossen.

Ebenso konnte die Facette „Autonomie“ der Überzeugung Schülerorientierung faktorenanalytisch abgebildet werden. Alle vier Items luden hier auf einem gemeinsamen Faktor. Ein Beispielitem lautet: „,... den Schülerinnen und Schülern Stoffe und Themen zur Auswahl zu geben“. Das Item, welches ursprünglich für die Dimension „Soziale Eingebundenheit" formuliert wurde, wurde mit einer Ladung von .54 dem Faktor 
„Autonomie“ zugeordnet: „... die Wünsche der Schülerinnen und Schülern soweit wie möglich zu erfüllen“.

Zwei Items der theoretisch postulierten Dimension „Soziale Eingebundenheit“ luden auf einem gemeinsamen Faktor. Ein Beispielitem lautet: „... mit Schülerinnen und Schülern zu reden“. Somit konnten alle drei theoretisch postulierten Facetten von Schülerorientierung faktorenanalytisch relativ gut abgebildet werden.

Alle fünf Items zur Dimension „Effektives Präsentieren“ luden auf einem gemeinsamen Faktor, wie zum Beispiel das Item ,... Lerninhalte sorgfältig und genau zu erklären“. Auch die vier Items der Dimension „Wiederholen, Zusammenfassen, Überprüfen“ luden auf einem Faktor, zum Beispiel das Item „... Lerninhalte regelmäßig wiederholen“. Ebenfalls luden die drei Items der Dimension „Vorführen, Zeigen, Demonstrieren“ auf einem Faktor, so wie das Item „...demonstrieren, wie Verfahren ausgeführt werden“. Das Item „,.. den Unterrichtsstoff regelmäßig zusammenzufassen, damit die Schülerinnen und Schüler sich diesen gut merken können“ wurde aufgrund seiner niedrigen Faktorladung von .42 ausgeschlossen, zudem wurde dieses Item ursprünglich für die Dimension „Wiederholen, Zusammenfassen, Überprüfen“ formuliert. Somit konnten alle drei theoretisch postulierten Facetten der Überzeugungen Transmission „Effektives Präsentieren“, „Vorführen, Zeigen, Demonstrieren“ und „Wiederholen, Zusammenfassen, Überprüfen“ mit den drei Faktoren abgebildet werden.

Drei Items der Dimension „Fehlerakzeptanz“, wie zum Beispiel das Item „... auch Fehler der Schülerinnen und Schüler zu akzeptieren und sie weitermachen zu lassen“ und das Item der Dimension „Selbstständiges und Selbstbestimmtes Lernen“ „... Schülerinnen und Schüler zu ermutigen, ihre eigenen Lösungswege zu suchen, auch wenn die ineffizient sind“ luden auf einem Faktor. Für diesen wurde die Bezeichnung „Fehlerakzeptanz“ gewählt. Auf einem gemeinsamen Faktor luden drei Items der Dimension „Selbstständiges und Selbstbestimmtes Lernen“, wie zum Beispiel das Item „...Zusammenhänge selbst entdecken können“. Auf dem nächsten Faktor luden drei Items der Überzeugung Konstruktion: „....es hinzunehmen, dass zum Lernen auch Fehler gehören, denn dadurch lernen Schülerinnen und Schüler überhaupt erst“, ,...Schülerinnen und Schüler zu ermutigen, ihre eigenen Wege zur Lösung von Aufgaben zu entdecken“ und „...Schülerinnen und Schüler zu ermutigen, sich selbst Lösungen auszudenken“. Diesen Items war der Gedanke gemeinsam, dass die Lehrkraft die Schülerinnen und Schüler ermutigt, auch eigene Lösungswege auszuprobieren. So wurde dieser Faktor „Individuelle Lösungswege“ genannt. Zusammenfassend konnten zwei der theoretisch postulierten Facetten der Überzeugung Konstruktion mit dieser Faktorlösung gut 
abgebildet werden. Zudem hat sich eine dritte Dimension herauskristallisiert. Zusammen werden folgende Dimensionen der Überzeugung Konstruktion unterschieden: „Fehlerakzeptanz“, „Selbstständiges und Selbstbestimmtes Lernen“ sowie die Dimension „Individuelle Lösungswege“"

In Bezug auf die theoretisch postulierten Facetten der Überzeugung Partizipation war die Faktorlösung weniger eindeutig. So luden auf einem Faktor die vier Items der Dimension „Kommunikation“ sowie das Item „....die Schülerinnen und Schüler gegenseitig Sachverhalte erklären und erläutern lassen“, und das Item zum gemeinsamen Lernen „...dass die Schülerinnen und Schüler sich gegenseitig beim Lernen unterstützen“. Dieser Faktor behielt die Bezeichnung „Kommunikation“. Die drei Items der Dimension „Lehrer in der Lerngemeinschaft" luden auf einem gemeinsamen Faktor. Diese zwei Facetten der Überzeugung Partizipation wurden faktorenanalytisch abgebildet.

Die übrigen Items fielen anders als erwartet zusammen. Drei Items luden auf einem Faktor: zwei aus der Dimension „Wertschätzung“ und eins aus „Gemeinschaftliches Lernen“: „... das Gemeinschaftsgefühl in der Klasse zu fördern“, „... das Wir-Gefühl in der Klasse zu stärken“ und „... dass Schülerinnen und Schüler gemeinsam lernen“. Alle diese Items beinhalteten den Gedanken der Gemeinschaft, deshalb wurde für diesen Faktor die neue Bezeichnung „Gemeinschaft“ gewählt. Andere sechs Items, die auf einem gemeinsamen Faktor luden, sprachen den Gedanken des gemeinsamen Wissens und gemeinschaftlichen Lernens an, entsprechend wurde dieser Faktor "Gemeinsames Wissen/gemeinschaftliches Lernen“ genannt. Ein Beispielitem hierfür lautet „gemeinsames Wissen in einer Lerngemeinschaft auszubauen“. Ein Item wurde aufgrund der geringen Faktorladung ausgeschlossen.

Weitere fünf Items luden auf einem Faktor, wobei zwei davon aufgrund ihrer geringen Ladungen von .39 und .38 ausgeschlossen wurden. Die drei Items, wie das Item „,...dass die Schülerinnen und Schüler in einen Diskurs über die Lerninhalte treten" - wurden zu einem Faktor „Diskutieren in einer Lerngemeinschaft" zusammengefasst. Somit konnten zwei der theoretisch postulierten Facetten der Partizipation durch die identifizierten Faktoren abgebildet werden: „Kommunikation“ und „Lehrer in der Lerngemeinschaft". Die Facetten „Gemeinschaftliches Lernen“ und „Gemeinsames Wissen“ sind in einem Faktor abgebildet, und ein neuer Faktor mit der Bezeichnung „Diskutieren in einer Lerngemeinschaft" wurde gebildet. Zwei Facetten der Partizipation „Teilhabe an der Gemeinschaft“ und „Wertschätzung“ konnten nicht wie erwartet abgebildet werden. 
Auf dieser Grundlage wurden mithilfe von Mittelwerten Skalen gebildet. Hierzu wurde definiert, dass bei einer Skala aus fünf Items mindestens vier Werte bei einer Versuchsperson vorhanden sein müssen, um in die Berechnung der Mittelwerte miteinbezogen zu werden bzw. zwei Werte bei einer Skala aus drei Items. Die so gebildeten Skalen besaßen hinreichende interne Konsistenzen:

- „Aufbau einer Beziehung zu Schülerinnen und Schülern“: $\alpha=.86$

- „Autonomie“: $\alpha=.80$

- „Soziale Eingebundenheit“": $\alpha=.57$

- Effektives Präsentieren“: $\alpha=.82$

- „Wiederholen, Zusammenfassen, Überprüfen“: $\alpha=.79$

- „Demonstrieren, Vorführen, Zeigen“: $\alpha=.76$

- „Fehlerakzeptanz“: $\alpha=.74$

- „Selbstständiges und Selbstbestimmtes Lernen“: $\alpha=.60$

- „Individuelle Lösungswege“: $\alpha=.59$

- „Kommunikation“: $\alpha=.76$

- „Lehrer in der Lerngemeinschaft“: $\alpha=.75$

- „Gemeinschaftlichkeit"“: $\alpha=.74$

- „Gemeinsames Wissen/gemeinschaftliches Lernen“: $\alpha=.82$

- „Diskutieren in einer Lerngemeinschaft"“: $\alpha=.66$

Zwei Items reduzierten die Reliabilitäten der gebildeten Skalen und wurden ausgeschlossen. Insgesamt wurde auf diese Weise der Itempool für die weitere Analyse auf 52 Items reduziert. Tabelle 7 zeigt die Mittelwerte und Standardabweichungen der Skalen sowie die Interkorrelationen zwischen den Skalen. 


\section{Tabelle 7}

Mittelwerte (M) und Standardabweichungen (SD) der Skalen sowie die Interkorrelationen zwischen den Skalen

\begin{tabular}{|c|c|c|c|c|c|c|c|c|c|c|c|c|c|c|c|}
\hline & Dimension & $\mathrm{M}(\mathrm{SD})$ & 2. & 3. & 4. & 5. & 6. & 7. & 8. & 9. & 10. & 11. & 12. & 13. & 14. \\
\hline \multirow{3}{*}{ 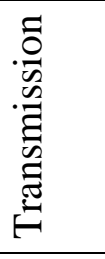 } & 1.Präsentieren & $3.56(.44)$ & $.50 * *$ & $.39 * *$ & .00 & $.25 * *$ & $.29 * *$ & $.21 *$ & $.16^{*}$ & $.25 * *$ & .11 & $.18^{*}$ & .06 & $.25 * *$ & $.27 * *$ \\
\hline & 2. Zeigen & $3.00(.57)$ & & $.33 * *$ & .20 & $.18^{*}$ & .02 & $.22 *$ & .08 & .16 & .16 & $.17 *$ & .10 & $.28 * *$ & .14 \\
\hline & 3.Wiederholen & $3.24(.48)$ & & & .08 & $.25^{* *}$ & $.21 * *$ & .12 & $.19 * *$ & $.21 * *$ & .13 & .03 & .07 & $.20 * *$ & .16 \\
\hline \multirow{3}{*}{ 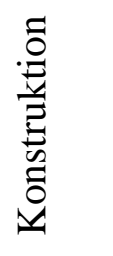 } & 4. Fehler & $2.82(.60)$ & & & & $.31 * *$ & $.40 * *$ & $.42 * *$ & .15 & $.30 * *$ & $.31 * *$ & $.36^{* *}$ & $.33 * *$ & $.27 * *$ & $.33 * *$ \\
\hline & 5. Selbstständig & $3.54(.44)$ & & & & & $.52 * *$ & $.53 * *$ & $.31 * *$ & $.52 * *$ & $.37 * *$ & $.19^{*}$ & $.28 * *$ & $.25^{* *}$ & $.32 * *$ \\
\hline & $\begin{array}{l}\text { 6. Individuelle } \\
\text { Lösungswege }\end{array}$ & $3.51(.43)$ & & & & & & $.46 * *$ & $.30 * *$ & $.48 * *$ & $.38 * *$ & $.33 * *$ & $.23 * *$ & $.33 * *$ & $.37 * *$ \\
\hline \multirow{5}{*}{ 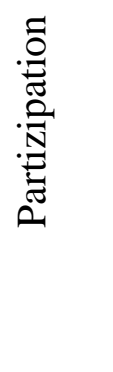 } & 7. Gemeinsames Wissen & $3.40(.44)$ & & & & & & & $.49 * *$ & $.59 * *$ & $.60 * *$ & $.46^{* *}$ & $.39 * *$ & $.37 * *$ & $.50 * *$ \\
\hline & 8. Gemeinschaft & $3.61(42)$ & & & & & & & & $.49 * *$ & $.42 * *$ & $.24 * *$ & $.31 * *$ & $.32 * *$ & $.34 * *$ \\
\hline & 9. Kommunikation & $3.47(.42)$ & & & & & & & & & $.55^{* *}$ & $.38 * *$ & $.39 * *$ & $.32 * *$ & $.31 * *$ \\
\hline & 10.Diskutieren & $3.25(.52)$ & & & & & & & & & & $.36^{* *}$ & $.43^{* *}$ & $.37 * *$ & $.43^{* *}$ \\
\hline & 11.Lehrer & $2.96(.61)$ & & & & & & & & & & & $.44 * *$ & $.26 * *$ & $.41 * *$ \\
\hline \multirow{3}{*}{ 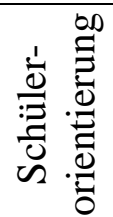 } & 12.Autonomie & $2.97(.56)$ & & & & & & & & & & & & $.42 * *$ & $.53 * *$ \\
\hline & 13.Eingebundenheit & $3.53(.46)$ & & & & & & & & & & & & & $.49 * *$ \\
\hline & 14.Beziehung & $3.21(.50)$ & & & & & & & & & & & & & \\
\hline
\end{tabular}


Erwartungsgemäß fielen die Interkorrelationen zwischen den einzelnen Facetten innerhalb einer Überzeugung höher aus als zwischen den Facetten verschiedener Überzeugungen. Auffallend waren die niedrigen, nicht signifikanten Korrelationswerte zwischen der Dimension „Fehlerakzeptanz“ der Überzeugung Konstruktion und allen Dimensionen von Transmission, ebenso wie die niedrigen Korrelationskoeffizienten zwischen der Dimension „Diskutieren“ der Überzeugung Partizipation und allen Dimensionen von Transmission sowie der „Schülerautonomie“ der Überzeugung Schülerorientierung. Dies könnte als ein Hinweis für die Distinktheit der Überzeugung Transmission und den anderen drei Überzeugungen gedeutet werden.

Zusammenfassend lässt sich festhalten, dass sich zehn der 14 angenommenen Facetten der vier Überzeugungen mittels der EFA relativ gut bestätigen ließen. Die gebildeten Indizes zeigten durchgehend akzeptable Reliabilitäten. Zudem ließen die niedrigen Interkorrelationswerte zwischen einzelnen Dimensionen von Konstruktion, Partizipation, Schülerorientierung und allen drei Dimensionen von Transmission eine Interpretation zu, sodass auf der Ebene der Facetten der vier Überzeugungen von deren Distinktheit ausgegangen werden kann.

Wie am Anfang erwähnt, sprach der Scree-Test für eine 4-Faktoren-Lösung; zudem wurde bei der Konstruktion des Fragebogens von vier Dimensionen ausgegangen. Daher sollte in einem zweiten Schritt die 4-Faktoren-Lösung einer genaueren Analyse unterzogen werden.

\section{Die 4-Faktoren-Lösung}

Mit den verbliebenen 52 Items wurde die Faktorenanalyse mit einer Extraktion von vier Faktoren wiederholt. Die vier Faktoren klärten 40.23 Prozent der Gesamtvarianz auf. In der rotierten Komponentenmatrix nach der Kaiser-Normalisierung zeichnete sich eine Faktorenstruktur ab, die die theoretisch angenommene Struktur mit den vier Überzeugungen Transmission, Konstruktion, Partizipation und Schülerorientierung gut abbildete und in fünf Iterationen konvergierte. 
Tabelle 8 gibt die Faktorladungsmatrix, die Bezeichnungen der gebildeten Skalen, die Anzahl der Items (n) und Cronbachs Alpha $(\alpha)$ der Skalen wieder.

\section{Tabelle 8}

Faktorladungsmatrix, gebildete Skalen (Partizipation - P; Schülerorientierung - SO;

Transmission - T; Konstruktion-K), Anzahl der Items (n) und Cronbachs Alpha ( $\alpha$ )

Unterrichten bedeutet für mich...

F $1 \quad$ F $2 \quad$ F $3 \quad$ F 4

... dass die Schülerinnen und Schüler einander fragen und gegenseitig diese Fragen beantworten.

$\begin{array}{llll}.73 & -.03 \quad-.00 \quad .12\end{array}$

... dass sich die Schülerinnen und Schüler über die Lerninhalte austauschen.

$\begin{array}{llll}.67 & .13 & .03 & -.09\end{array}$

... die Schülerinnen und Schüler gegenseitig Sachverhalte erklären und erläutern zu lassen.

$\begin{array}{llll}.63 & -.02 & .16 & .17\end{array}$

... dass die Schülerinnen und Schüler sich gegenseitig beim Lernen unterstützen.

$\begin{array}{llll}.63 & .22 & .16 & -.01\end{array}$

... das Wir-Gefühl in der Klasse zu fördern.

$\begin{array}{llll}.62 & .32 & .10 & -.13\end{array}$

... das gemeinschaftlich geteilte Wissen in der Gruppe zu entwickeln.

$\begin{array}{llll}.60 & .18 & .07 \quad .23\end{array}$

...gemeinsames Wissen in einer Lerngemeinschaft aufzubauen.

$\begin{array}{llll}.56 & .34 & .17 \quad .28\end{array}$

... dass die Schülerinnen und Schüler voneinander lernen.

$\begin{array}{llll}.55 & .26 \quad .02 \quad .22\end{array}$

... in Lerngemeinschaft das Wissen zu teilen.

$.55 \quad .28 \quad .00 \quad .06$

... das gemeinschaftliche Lernen unter den Schülerinnen und Schülern zu fördern.

$\begin{array}{llll}.54 & .34 & .05 & .05\end{array}$

... Schülerinnen und Schüler zu ermutigen, selbst zu $\begin{array}{llllll}\text { entdecken, wie Aufgaben bearbeitet werden können.* } & .53 & -.01 & .12 & .13\end{array}$ 
...zum Aufbau einer Lerngemeinschaft beizutragen.

$\begin{array}{llll}.52 & .33 & -.03 \quad .08\end{array}$

... Schülerinnen und Schüler zu ermutigen, ihre eigenen

Wege zur Lösung von Aufgaben zu entdecken.

... das Gemeinschaftsgefühl in der Klasse zu fördern.

$\begin{array}{llll}.46 & .20 & .06 & -.21\end{array}$

... dass die Schülerinnen und Schüler das, was nicht

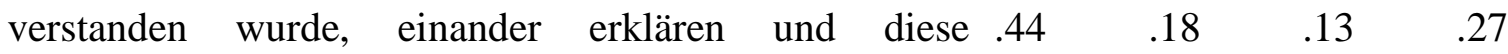

Erklärungen auch einfordern.

... Schülerinnen und Schüler zu ermutigen, sich selbst Lösungen auszudenken.

$\begin{array}{llll}.43 & .16 & .17 & .36\end{array}$

.. das Stoffgebiet so zu vermitteln, dass Schülerinnen und Schüler Zusammenhänge selbst entdecken können.

... dass die Schülerinnen und Schüler in einen Diskurs über die Lerninhalte treten.

... geeignete Materialien einzusetzen, damit $\begin{array}{llllll}\text { Schülerinnen und Schüler selbst Wege zur Lösung einer } & .40 & .09 & .16 & .19\end{array}$ Aufgabe entwickeln.

...obwohl Experte, ein Mitglied der Lerngemeinschaft mit gleichen Orientierungen zu sein.***

... zu den Schülerinnen und Schülern eine Beziehung $\begin{array}{llllll}\text { aufzubauen, sodass die Lehrperson ihre Schülerinnen und } & -.02 & .73 & .08 & .07\end{array}$ Schüler kennt.

... die Beziehungen zu den Schülerinnen und Schülern $\begin{array}{lllllllll}\text { zu stärken, um die Schülerpersönlichkeiten } & \text { gut } & \text { zu } & .15 & & .69 & .14 & & .09\end{array}$ kennen und diese als Personen wahrnehmen zu können.

... die Lebenswelt der Schülerinnen und Schüler mit

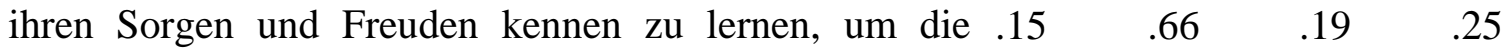
Lernprozesse besser verstehen zu können.

... die Schülerinnen und Schüler besser zu kennen, damit diese Kenntnisse der Lehrperson helfen, das Lernen der Schülerinnen und Schüler in einem realen Kontext $\mathrm{zu}$ $\begin{array}{llll}.11 & .64 & .11\end{array}$ verstehen.

... den Schülerinnen und Schülern Stoffe und Themen zur Auswahl zu geben. 
... die Schülerinnen und Schüler selbst darüber bestimmen $\mathrm{zu}$ lassen, mit welchen Themen sie sich .15 beschäftigen möchten.

... zu den Schülerinnen und Schülern eine Beziehung aufzubauen, damit sie die Lehrperson als einen Menschen auffassen und von ihr als Personen aufgefasst werden.

... bei der Stoffauswahl auf individuelle Wünsche der Schülerinnen und Schülern einzugehen.

... die Wünsche der Schülerinnen und Schüler soweit wie möglich zu erfüllen.

... die Schülerinnen und Schüler besser kennen zu lernen, um ihre Lernergebnisse in den Kontext ihrer Lebenssituation einordnen und dadurch besser verstehen zu können.

... sich als Lehrkraft auch um die Probleme der Schülerinnen und Schüler zu kümmern.

$\begin{array}{llll}.23 & .54 & .27 \quad-.04\end{array}$

... darauf zu achten, dass jede und jeder Einzelne ein Teil einer Lerngemeinschaft werden kann.

... bei der Themenwahl die Interessen der Schülerinnen und Schüler einzubeziehen.

... mit den Schülerinnen und Schülern zu reden, wenn ihnen etwas nicht gefällt.

... obwohl Experte, ein Mitglied der Lerngemeinschaft

mit gleichen Orientierungen zu sein. **

... Lerninhalte zu präsentieren, sodass diese von den

Schülerinnen und Schülern gut aufgenommen werden .09

können.

...Lerninhalte für die Schülerinnen und Schülern gut nachvollziehbar zu referieren.

... Lerninhalte sorgfältig zu erläutern, sodass die Schülerinnen und Schüler sich diese gut merken können.

... Lerninhalte $\mathrm{zu}$ präsentieren, sodass sie für die Schülerinnen und Schüler gut nachvollziehbar sind. 
... den Schülerinnen und Schülern zu demonstrieren, wie

Verfahren ausgeführt werden.

$\begin{array}{llll}.03 & .15 & .60 & .01\end{array}$

... Vorgehensweisen zu zeigen, damit sich die

Schülerinnen und Schüler daran orientieren können.

$\begin{array}{llll}.01 & .11 \quad .60 & -.14\end{array}$

... das Gelernte regelmäßig zu überprüfen, damit das

$\begin{array}{llllll}\text { Wissen der Schülerinnen und Schüler gefestigt werden } & .04 & -.13 & .59 & .12\end{array}$

kann.

... Lerninhalte regelmäßig zu überprüfen, um

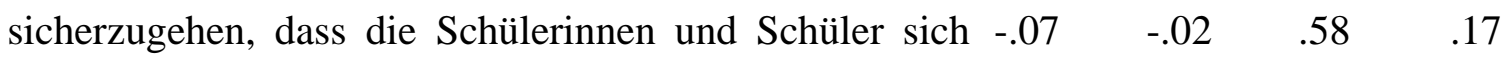

diese gut eingeprägt haben.

... Lerninhalte sorgfältig und genau zu erklären.

$.07 \quad .05 \quad .58$

.11

... regelmäßige Übungsaufgaben zu vergeben, die den

$\begin{array}{lllllllll}\text { Schülerinnen und Schülern helfen, das Gelernte } \mathrm{zu} & .13 & -.01 & .58 & .25\end{array}$

festigen.

...Arbeitsschritte vorzuführen, damit die Schülerinnen

und Schüler diese gut nachahmen können.

$\begin{array}{llll}.07 & .14 & .55 & -.16\end{array}$

... Lerninhalte regelmäßig zu wiederholen, damit die

$\begin{array}{lllllll}\text { Schülerinnen und Schüler sich diese gut einprägen } & .09 & .03 & .50 & .22\end{array}$

können.

... die Schülerinnen und Schüler mit ihren eigenen

$\begin{array}{llllll}\text { Ergebnissen auch einmal bewusst in die Irre laufen } \mathrm{zu} & -.02 & .12 & -.02 & .73\end{array}$

lassen, bis sie es selbst gemerkt haben.

... auch Fehler der Schülerinnen und Schüler zu

akzeptieren und sie selbst weitermachen zu lassen.

... Schülerinnen und Schüler zu ermutigen, ihre eigenen

Lösungswege zu suchen, auch wenn sie ineffizient sind.

$.17 \quad .27 \quad .01 \quad .60$

... auch Fehler der Schülerinnen und Schüler zu

$\begin{array}{lllll}\text { akzeptieren und sie weitermachen zu lassen, bis sie selbst } & .26 & .09 & -.12 & .60\end{array}$

gemerkt haben, dass etwas nicht stimmt.

... es hinzunehmen, dass zum Lernen auch Fehler

$\begin{array}{lllllll}\text { gehören, denn dadurch lernen die Schülerinnen und } & .23 & .10 & .18 & .35\end{array}$

Schüler überhaupt erst.

\begin{tabular}{lllll}
\hline Gebildete Skalen & $\mathrm{P}$ & $\mathrm{SO}$ & $\mathrm{T}$ & $\mathrm{K}$ \\
\hline Anzahl der Items $(\mathrm{n})$ & 14 & 13 & 12 & 5 \\
\hline Cronbachs $\alpha$ & .84 & .88 & .85 & .72 \\
\hline
\end{tabular}




\footnotetext{
*Kursiv und fett markierte Items wurden aufgrund nicht theoriekonformer Faktorladungen ausgeschlossen.

**Kursiv markierte Items wurden aufgrund geringer Faktorladung ausgeschlossen.

***Fett markierte Items wurden ausgeschlossen, weil sich bei Beibehaltung die Reliabilität der Skala verschlechterte.
}

Mit wenigen Ausnahmen luden alle Items theoriekonform auf dem entsprechenden Faktor. So lud zum Beispiel das zur Transmission formulierte Item „... Lerninhalte für die Schülerinnen und Schülern gut nachvollziehbar zu referieren“ mit einer Faktorladung von .67 auf dem Faktor „Transmission“ und das zur Konstruktion formulierte Item „... die Schülerinnen und Schüler mit ihren eigenen Ergebnissen auch einmal bewusst in die Irre laufen zu lassen, bis sie es selbst gemerkt haben“ mit einer Faktorladung von .73 auf dem Faktor „Konstruktion“. Das Item zur Schülerorientierung „.. die Lebenswelt der Schülerinnen und Schülern mit ihren Sorgen und Freuden besser kennen zu lernen, um die Lernprozesse besser verstehen zu können“ lud mit einer Faktorladung von .66 auf dem entsprechenden Faktor. Hier wurde ein ursprünglich zur Partizipation formuliertes Item „,.. jede/r einzelne Schüler/in Teil einer Lerngemeinschaft wird“ aufgrund seiner nicht theoriekonformen Ladung aus weiteren Analysen ausgeschlossen. Theoriekonforme Ladungen zeigten eine Reihe von Items zur Partizipation: zum Beispiel das Item „...das gemeinschaftlich geteilte Wissen in der Gruppe zu entwickeln“ mit einer Faktorladung von .60. Andererseits luden einige zur Konstruktion formulierte Items auf dem Faktor „Partizipation“ (z.B. „...geeignete Materialien einzusetzen, damit Schülerinnen und Schüler selbst Wege zur Lösung einer Aufgabe entwickeln“). Diese wurden aufgrund der nicht theoriekonformen Ladungen aus den Analysen ausgeschlossen. Ein Item wurde ausgeschlossen, weil es die Reliabilität der Skala verschlechterte. Zusätzlich wurde diese 4Faktoren-Lösung auf heterogene Faktorladungen überprüft: Alle Items ließen sich eindeutig einem Faktor zuordnen. Eine für die Zuordnung zu einem Faktor ausreichende Faktorladung wurde bei .35 angesetzt. Nur ein Item wurde aufgrund seiner geringen Faktorladung ausgeschlossen. Insgesamt wurden acht Items ausgeschlossen, sodass die anschließenden Analysen mit 44 Items durchgeführt wurden.

Bei einer erneuten Durchführung der Faktorenanalyse mit der reduzierten Itemzahl erklärten die vier Faktoren 42.77 Prozent der Gesamtvarianz. Eine Tabelle mit den Mittelwerten, Standardabweichungen und Trennschärfen dieser Items kann im Anhang B angesehen werden. Tabelle 9 zeigt die Mittelwerte und Standardabweichungen der Skalen sowie die Interkorrelationen zwischen den Skalen. 


\section{Tabelle 9}

Mittelwerte $(M)$ und Standardabweichungen $(S D)$ und Interkorrelationen der Skalen

\begin{tabular}{lllll}
\hline & $M(S D)$ & Schülerorientierung & Transmission & Konstruktion \\
\hline Partizipation & $3.42(.37)$ & $.58^{*}$ & $.31^{*}$ & $.41^{*}$ \\
Schülerorientierung & $3.17(.44)$ & & $.22^{*}$ & $.37^{*}$ \\
Transmission & $3.31(.38)$ & & & .10 \\
Konstruktion & $3.05(.46)$ & & & \\
\hline$* p<.01$. & & & &
\end{tabular}

Es zeigten sich deskriptive Unterschiede zwischen den Mittelwerten der vier Skalen sowie folgende Korrelationsmuster: Während die Korrelationskoeffizienten zwischen Partizipation und Schülerorientierung $(r=.58, p<.01)$ sowie zwischen Partizipation und Konstruktion $(r=.41, p<.01)$, aber auch zwischen Konstruktion und Schülerorientierung $(\mathrm{r}=$ $.37, p<.01)$ im mittleren Bereich liegen, zeigen sich niedrigere Korrelationskoeffizienten zwischen Transmission und Partizipation $(r=.31, p<.01)$ sowie zwischen Transmission und Schülerorientierung $(r=.22, p<.01)$. Transmission und Konstruktion $(r=.10, p=.18)$ korrelierten nur schwach und nicht signifikant miteinander.

Diese Korrelationsmuster zeigen, dass die Überzeugungen Partizipation, Konstruktion und Schülerorientierung in einer engen Beziehung zueinander stehen. Dagegen ist die Verbindung von Transmission zu Partizipation und Schülerorientierung weniger ausgeprägt. Zudem scheinen die beiden Überzeugungen Transmission und Konstruktion unabhängig voneinander zu sein, da sie nicht miteinander korrelieren. Somit setzt sich Transmission von den anderen drei Überzeugungen ab.

\subsubsection{Konstruktvalidierung}

Zusammenhänge zwischen den Skalen der vier Überzeugungen zum Lehren und Lernen und den Skalen zu Lern-/Lehrbegriffen sowie Erziehungszielen

Zur Konstruktvalidierung des neu entwickelten Fragebogens wurden korrelative Zusammenhänge zwischen den vier gebildeten Skalen und den Lern- und Lehrbegriffsskalen sowie der Skala zu Erziehungszielen berechnet (siehe Tab. 10). Dabei wurden folgende Zusammenhänge erwartet: Es sollten sich positive Korrelationen zwischen der Überzeugung Konstruktion und dem Lernbegriff „Verstehen“ sowie dem Lehrbegriff „Lernbegleitungsorientiert“ zeigen, da die Überzeugung Konstruktion mit der Vorstellung 
vom Lernen als einem aktiven Verstehensprozess und mit der des Lehrens als Unterstützung von Lernprozessen einhergeht. Weiterhin kann eine engere Beziehung zwischen Transmission und Erziehungszielen, die auf Leistung und auf Ordnung und Disziplin abzielen, erwartet werden sowie zwischen Partizipation und den Erziehungszielen zu sozial angemessenen Umgangsformen und sozialem Verhalten. Weitere Annahmen können beim heutigen Forschungsstand nicht theoretisch gestützt werden.

\section{Tabelle 10}

Korrelationen zwischen den vier Skalen der Überzeugungen zum Lehren und Lernen und den Lern- und Lehrbegriffen sowie Erziehungszielen

\begin{tabular}{|c|c|c|c|c|}
\hline & Partizipation & $\begin{array}{l}\text { Schüler- } \\
\text { orientierung }\end{array}$ & Transmission & Konstruktion \\
\hline \multicolumn{5}{|l|}{ Lernbegriff } \\
\hline Lernen ist Explorieren & .15 & .15 & -.01 & .15 \\
\hline Lernen ist Verstehen & $.40 * *$ & $.21 *$ & .18 & $.26^{*}$ \\
\hline \multicolumn{5}{|l|}{ Lehrbegriff } \\
\hline Instruktionsorientiert & .07 & .13 & $.29 * *$ & -.05 \\
\hline Lernbegleitungsorientiert & $.37 * *$ & $.39 * *$ & .18 & $.36 * *$ \\
\hline \multicolumn{5}{|l|}{ Erziehungsziele } \\
\hline $\begin{array}{l}\text { Leistungs- und } \\
\text { Anstrengungsbereitschaft }\end{array}$ & .13 & .01 & $.20^{*}$ & .05 \\
\hline Ordnung und Disziplin & .06 & -.06 & $.30 * *$ & .03 \\
\hline $\begin{array}{l}\text { Solide Kenntnisse in den } \\
\text { Hauptfächern }\end{array}$ & .06 & -.12 & $.21 *$ & -.13 \\
\hline $\begin{array}{l}\text { Soziale } \\
\text { Verantwortungsbereitschaft }\end{array}$ & $.21 *$ & .14 & .06 & .08 \\
\hline $\begin{array}{l}\text { Angemessene soziale } \\
\text { Umgangsformen }\end{array}$ & $.24 *$ & .15 & .10 & .10 \\
\hline Hilfsbereites Verhalten & $.20^{*}$ & .17 & .14 & .07 \\
\hline $\begin{array}{l}\text { Kenntnisse, die auf den Beruf } \\
\text { vorbereiten }\end{array}$ & $.25^{*}$ & .17 & .13 & .14 \\
\hline
\end{tabular}


Signifikante Korrelationen zwischen Lehr-Lern-Begriffen und den Überzeugungen Transmission und Konstruktion bestätigen die Annahmen, dass Lernen nach einer konstruktivistischen Vorstellung dem Verstehen gleichkommt und dass Lehren der Lernbegleitung dient. Die Konstruktion wies einen signifikanten positiven Zusammenhang mit dem Begriff des Lernens als Verstehen auf: $r=.26, p<.05$ und mit dem Lehrbegriff als Lernbegleitung: $r=.36, p<.01$. Dagegen hängt die Überzeugung Transmission mit einem instruktionsorientierten Lehrbegriff zusammen $(r=.29, p<.01)$. Zudem zeigten sich positive signifikante Korrelationen der Transmission mit „Leistungs- und Anstrengungsbereitschaft“ ( $r=.20, p<.05)$, mit „Ordnung und Disziplin“ ( $r=.30, p<.01)$ und mit ,,soliden Kenntnissen in den Hauptfächern“ ( $r=.21, p<.05)$.

Die Überzeugungen Partizipation und Schülerorientierung scheinen ähnliche Beziehungen zu den Lern-/Lehrbegriffen aufzuweisen wie die Überzeugung Konstruktion. Partizipation hängt signifikant positiv mit „Lernen als Verstehen“ $(r=.40, p<.01)$ und mit dem lernbegleitungsorientierten Lehrbegriff $(r=.37, p<.01)$ zusammen. Schülerorientierung hängt signifikant positiv mit „Verstehen“ $(r=.21, p<.05)$ und mit dem lernbegleitungsorientierten Lehrbegriff $(r=.39, p<.01)$ zusammen. Die berichteten Zusammenhänge bestätigen die im Voraus formulierten Annahmen weitestgehend.

Zusammenhänge zwischen den Skalen zu Überzeugungen zum Lehren und Lernen und den Skalen zu epistemologischen Überzeugungen

Zur Konstruktvalidierung des neu entwickelten Instruments Q4TB wurden weiterhin Korrelationen zwischen den vier Skalen des Q4TB und den vier Skalen zu allgemeinen epistemologischen Überzeugungen (siehe Tab. 11) berechnet. Es wurde erwartet, dass Transmission mit einer naiveren epistemologischen Überzeugung, also mit Dualismus, sowie Konstruktion mit einer elaborierteren Überzeugung wie Relativismus zusammenhängen. 


\section{Tabelle 11}

Korrelationskoeffizienten zwischen den Skalen Dualismus und Relativismus und den vier lehr-/lernbezogenen Überzeugungen - Partizipation, Schülerorientierung, Transmission und Konstruktion

\begin{tabular}{lllll}
\hline & Partizipation & Schülerorientierung & Transmission & Konstruktion \\
\hline Dualismus & .04 & .18 & $.13^{*}$ & .10 \\
Relativismus & $.12 *$ & .05 & .02 & $.13^{*}$ \\
\hline$* p<.05$. & & &
\end{tabular}

Die Interkorrelationen bestätigen die formulierten Erwartungen: Transmission hängt positiv mit der Skala Dualismus der epistemologischen Überzeugungen $(r=.13, p<.05)$ zusammen, die für eine naivere Wissenschaftsgläubigkeit steht. Dagegen zeigte die LehrLern-Überzeugung Konstruktion eine positive signifikante Korrelation mit der Skala Relativismus ( $r=.13, p<.05)$, die einen kritischen Umgang mit wissenschaftlichen Theorien als menschliche Konstruktionen repräsentiert. Auch für die Überzeugung Partizipation bestätigte sich der erwartete Zusammenhang: Diese Überzeugung hängt signifikant positiv mit der Skala wissenschaftskritischen Relativismus zusammen: $r=.12, p<.05$. Insgesamt fielen die Korrelationskoeffizienten in einem niedrigen Bereich aus, was auf schwache Verbindungen zwischen den vier Skalen der Lehr-Lern-Überzeugungen und den Skalen zu epistemologischen Überzeugungen schließen lässt.

Geschlechts- und fächerkombinationsspezifische Unterschiede in den Überzeugungen zum Lehren und Lernen

Für die Untersuchung der Sensitivität des neu entwickelten Fragebogens gegenüber geschlechtsspezifischen und fächerkombinationsspezifischen Unterschieden wurde eine mehrfaktorielle multivariate Varianzanalyse berechnet. Die Variable Geschlecht zeigte naturgemäß zwei Ausprägungen - weiblich und männlich. Um fächerkombinationsspezifische Unterschiede zwischen den Überzeugungen zum Lehren und Lernen zu ermitteln, wurden die Teilnehmer nach ihren studierten Fächern in drei Gruppen aufgeteilt: Die „Naturwissenschaften“ bildete die Gruppe der Studierenden, bei denen beide Studienfächer aus der Gruppe der Naturwissenschaften wie Biologie, Mathematik oder Chemie stammten. Die „Geisteswissenschaften“ umfassten Studierende, die Studienfächer aus den Geisteswissenschaften wie Geschichte, Theologie oder Englisch gewählt hatten, und die 
„gemischte Gruppe“ Studierende, die sowohl ein naturwissenschaftliches als auch ein geisteswissenschaftliches Fach studierten. Somit konnte der Einfluss der Fächerkombination und des Geschlechts auf die vier abhängigen Variablen - die vier Überzeugungen - getestet werden. Anschließend wurden mit dem paarweisen Gruppenvergleich mittels des Tukey-Tests die Unterschiede zwischen den einzelnen Gruppen „Naturwissenschaften“, „Geisteswissenschaften“ und „Gemischte Gruppe“ analysiert.

Die Mittelwerte und Standardabweichungen der vier Skalen für die Studentinnen und Studenten sowie für die drei Fächerkombinationsgruppen sind in Tabelle 12 dargestellt.

\section{Tabelle 12}

Mittelwerte (M) und Standardabweichungen (SD) der Skalen in Abhängigkeit vom Geschlecht und von der studierten Fächerkombination

\begin{tabular}{|c|c|c|c|c|c|c|c|c|c|c|}
\hline & \multicolumn{4}{|c|}{ Geschlecht } & \multicolumn{6}{|c|}{ Fächerkombination } \\
\hline & \multicolumn{2}{|c|}{$\begin{array}{l}\text { Weiblich } \\
(n=133)\end{array}$} & \multicolumn{2}{|c|}{$\begin{array}{l}\text { Männlich } \\
(n=48)\end{array}$} & \multicolumn{2}{|c|}{$\begin{array}{l}\text { Naturwis- } \\
\text { senschaften } \\
(\mathrm{n}=15)\end{array}$} & \multicolumn{2}{|c|}{$\begin{array}{l}\text { Geisteswissen- } \\
\text { schaften } \\
(n=116)\end{array}$} & \multicolumn{2}{|c|}{$\begin{array}{l}\text { Gemischte } \\
\text { Gruppe } \\
(n=48)\end{array}$} \\
\hline & $M$ & SD & $\mathrm{M}$ & SD & $\mathrm{M}$ & SD & $\mathrm{M}$ & SD & $\mathrm{M}$ & SD \\
\hline Partizipation & $3.48 *$ & .36 & $3.35 *$ & .37 & 3.52 & .38 & 3.46 & .37 & 3.35 & .38 \\
\hline Konstruktion & 3.04 & .47 & 3.08 & .50 & $3.41 *$ & .34 & $3.00 *$ & .47 & $3.06 *$ & .43 \\
\hline Transmission & 3.34 & .39 & 3.30 & .37 & 3.37 & .43 & 3.34 & .38 & 3.30 & .36 \\
\hline $\begin{array}{l}\text { Schülerorien- } \\
\text { tierung }\end{array}$ & 3.18 & .43 & 3.17 & .47 & 3.24 & .63 & 3.19 & .40 & 3.12 & .47 \\
\hline
\end{tabular}

Zum einen wurde ein signifikanter Effekt der Geschlechtsvariable auf die vier Überzeugungen festgestellt $(F(4 ; 165)=2.09, p<.10 ; \eta 2=.05)$. Der signifikante Unterschied bestand in der Skala Partizipation $(F(1)=5.29, p<.05, \eta 2=.03)$ : Studentinnen weisen signifikant höhere Werte in Partizipation auf als Studenten.

Zum anderen wurde ein signifikanter Effekt der Fächerkombination auf die vier Überzeugungen ermittelt $(F(4,166)=2.41, p<.05, \eta 2=.06)$. Der signifikante Unterschied wurde in der Skala Konstruktion ermittelt $(F(2)=4.53, p<.05, \eta 2=.05)$. Der Gruppenvergleich ergab, dass die Gruppe der Naturwissenschaften verglichen mit der Gruppe der Geisteswissenschaften signifikant höhere Werte in Konstruktion aufwies (Cohen's d = .99) und ebenfalls verglichen mit der gemischten Gruppe höhere Werte zeigte (Cohen's d = .90). Allerdings muss auf die kleine Stichprobengröße der Gruppe der Naturwissenschaften 
hingewiesen werden. Da es in der Gruppe nur 15 Studierende der Naturwissenschaften gab, ist die Testpower stark eingeschränkt.

Zusammenfassend lässt sich sagen, dass der entwickelte Fragebogen sensitiv gegenüber Geschlechts- sowie Fächerkombinationseffekten zu sein scheint. Mit dem Fragebogen ist es demnach partiell möglich, die Unterschiede in den Überzeugungen zum Lehren und Lernen zwischen weiblichen und männlichen Probanden sowie zwischen Studierenden unterschiedlicher Fächer aufzudecken.

Unterschiede in den Überzeugungen zum Lehren und Lernen zwischen Bachelor- und Masterstudierenden

Beim Vergleich der Bachelor- mit Masterstudierenden wurden Unterschiede erwartet. So sollten Masterstudierende aufgrund ihrer im Studium angeeigneten lehr-/lerntheoretischen Kenntnisse die Präferenz für die Überzeugung Transmission zugunsten fortgeschrittener Überzeugungen wie Konstruktion oder Partizipation aufgeben. Die Mittelwerte und Standardabweichungen der beiden Gruppen in den vier Überzeugungen sind in Tabelle 13 angegeben.

\section{Tabelle 13}

Mittelwerte (M) und Standardabweichungen (SD) der Skalen für Bachelor- und Masterstudierende

\begin{tabular}{lllll}
\hline & \multicolumn{2}{l}{ Bachelorstudierende $(n=145)$} & \multicolumn{2}{l}{ Masterstudierende $(n=50)$} \\
\cline { 2 - 5 } & $\mathrm{M}$ & $\mathrm{SD}$ & $\mathrm{M}$ & $\mathrm{SD}$ \\
\hline Partizipation & $3.40^{*}$ & .37 & $3.53^{*}$ & .35 \\
Konstruktion & 3.04 & .48 & 3.07 & .43 \\
Transmission & $3.37^{* *}$ & .35 & $3.19^{* *}$ & .41 \\
Schülerorientierung & 3.13 & .43 & 3.26 & .44 \\
\hline
\end{tabular}

$* * p<.01, * p<.05$

Die deskriptiven Unterschiede zwischen den Bachelor- und Masterstudierenden wurden mittels einer multivariaten Varianzanalyse (MANOVA) auf ihre Signifikanz hin überprüft. Entsprechend den Erwartungen zeigte sich ein signifikanter Effekt des Faktors „Abschluss“: Bachelor- und Masterstudierende unterscheiden sich voneinander in ihren Überzeugungen $(F(4,190)=5.42, p<.01, \eta 2=.10)$. Masterstudierende zeigten signifikant 
höhere Werte in der Überzeugung Partizipation $(F(1)=4.40, p<.05, \eta 2=.02$ ) sowie signifikant niedrigere Werte in Transmission $(F(1)=9.44, p<.01 ; \eta 2=.05)$.

Es kann festgehalten werden, dass Lehren und Lernen von Masterstudierenden im Vergleich zu Bachelorstudierenden eher entsprechend den partizipatorischen Überzeugungen verstanden werden. Masterstudierende besitzen weniger transmissive Überzeugungen als Studierende am Anfang des Studiums (Bachelor). 


\subsection{Diskussion}

Das primäre Ziel dieser Studie lag in der Entwicklung eines Instruments zur Erfassung der vier Lehrerüberzeugungen zum Lehren und Lernen. Die zentrale Fragestellung lautete, inwieweit es gelingen kann, die Überzeugungen zum Lehren und Lernen - Transmission, Konstruktion, Partizipation und Schülerorientierung - mit einem quantitativen Instrument zu erfassen, wobei versucht wurde, über die bisherige Forschung hinauszugehen, indem partizipatorische und schülerorientierte Überzeugungen mit quantitativen Methoden gemessen wurden. Dabei wurden die Überzeugungen Transmission und Konstruktion in Orientierung an bestehende Instrumente (z.B. BIJU; Daniels, 2008; COACTIV; Baumert et al., 2008; Staub \& Stern, 2002) untersucht. Die Überzeugungen Partizipation und Schülerorientierung wurden in Orientierung an den theoretischen Lerngemeinschaftsansatz (Lave \& Wenger, 1991) sowie das Konstrukt der Schülerorientierung (Klieme et al., 2006) verstanden. Zusätzliche Hilfe bei der Itemformulierung der Überzeugung Partizipation leistete die im Vorfeld der Fragebogenentwicklung durchgeführte Interviewstudie. Diese konnte in einem ersten Schritt bestätigen, dass die vier Lehr-Lern-Überzeugungen in den Vorstellungen der befragten Lehrkräfte präsent sind. Hinsichtlich der Überzeugung Partizipation wurden inhaltsanalytisch differenzierende Kategorien mit Ankerbeispielen extrahiert, welche für die Formulierung der Items verwendet werden konnten. Der so entwickelte Fragebogen wurde an einer Stichprobe von Lehramtsstudierenden zu Beginn des Bachelorstudiums und im dritten Semester des Masterstudiums untersucht. Für die Lehr-Lern-Überzeugung Schülerorientierung konnten Skalen aus der BIJU-Studie verwendet werden, die die gleichen Ideen beinhalten wie die Facetten der Überzeugung. 


\subsubsection{Faktorenstruktur}

Die Ergebnisse der faktorenanalytischen Prüfung bestätigten recht eindrucksvoll die Struktur, die der Konstruktion des Fragebogens zugrunde lag. Empirisch ergaben sich Hinweise sowohl auf eine Lösung mit 14 Faktoren als auch auf eine Lösung mit vier Faktoren. Die 14-Faktoren-Lösung bildete relativ gut die angenommenen Facetten der Überzeugungen $a b$, wie beispielsweise „Effektives Präsentieren“ der Überzeugung Transmission oder „Diskutieren in einer Lerngemeinschaft“ der Überzeugung Partizipation. Alle in dieser Lösung gebildeten Skalen wiesen zufriedenstellende interne Konsistenzen auf: Cronbachs Alphas der Skalen lagen im Bereich zwischen $\alpha=.57$ und $\alpha=.86$. Es wurde neben der 14-Faktoren-Lösung die 4-Faktoren-Lösung analysiert, welche die vier angenommenen Überzeugungen Transmission, Partizipation, Konstruktion und Schülerorientierung mit Reliabilitäten zwischen $\alpha=.72$ und $\alpha=.88$ gut abbildete. Damit ist es möglich, Lehrerüberzeugungen zum Lehren und Lernen sowohl anhand der vier übergeordneten Überzeugungen wie auch auf der Grundlage spezifischer Facetten zu erfassen.

Bei der Betrachtung der Interkorrelationsmuster zwischen den vier Skalen fielen einerseits die etwas höheren bivariaten Korrelationen zwischen den Skalen Partizipation, Schülerorientierung und Konstruktion auf. Andererseits lagen die Korrelationskoeffizienten zwischen Transmission und Partizipation sowie Transmission und Schülerorientierung im mittleren Bereich, während Transmission und Konstruktion nicht miteinander assoziiert waren. Dies ist als Hinweis darauf zu deuten, dass sich Transmission von Partizipation und Schülerorientierung absetzt bzw. die drei Überzeugungen einander näher stehen als dies zu Transmission der Fall ist. Während Transmission den Fokus auf Lerninhalte legt, betonen die anderen drei Überzeugungen den Schüler mit seinen individuellen Lernprozessen, die LehrerSchüler-Beziehung oder die Lerngemeinschaft. Denkbar wäre, dass diese engere Beziehung zwischen den dreien auf eine übergeordnete Überzeugungsdimension hindeutet. Die Struktur der Überzeugungen ließe sich dann in zwei distinkten Lehr-Lern-Vorstellungen höherer Ordnung verstehen - auf der einen Seite die lerninhaltsbezogene Transmission und auf der anderen Seite eine Vorstellung, bei der das Lernen nicht aus der Sicht des Lehrers und mit Fokussierung auf den „Lernstoff“, sondern aus der Schülersicht betrachtet wird: Was geht im Kopf des Schülers vor (Konstruktion)? Welche Lernereignisse sind in der Lerngemeinschaft wichtig (Partizipation) und wie kann die Beziehung des Lehrers zum Schüler aussehen (Schülerorientierung), um entsprechend der Schülerpersönlichkeit das Lernen zu fördern? Dies wären mögliche Fragestellungen zukünftiger Forschung. An dieser Stelle kann 
festgehalten werden, dass es sich bei den vier Dimensionen um empirisch voneinander abgetrennte Überzeugungen handelt.

\subsubsection{Konstruktvalidität}

Die konvergente Validität des entwickelten Fragebogens wurde mittels korrelativer Zusammenhänge mit konstruktnahen Skalen überprüft. Signifikante Korrelationen zwischen den vier Überzeugungen und den Lern-/Lehrbegriffen, den Erziehungszielen sowie den allgemeinen epistemologischen Überzeugungen bestätigten die formulierte Annahmen. Die Überzeugung Transmission ging mit einem instruktionsorientierten Lehrbegriff einher und mit den Erziehungszielen Leistung, Kenntnissen und Disziplin. Dagegen zeigte sich, dass die konstruktivistische Lehr-Lern-Überzeugung dem Lernen als Verstehen gleichkommt und Lehren als Lernbegleitung auffasst. Zudem gingen Partizipation und Schülerorientierung mit Lehr-Lern-Begriffen einher: Nach der partizipatorischen Lehr-Lern-Überzeugung bedeutet Lernen Verstehen, und nach der schülerorientierten heißt Lehren Lernbegleitung. Zudem sind soziale Aspekte wie angemessene Umgangsformen und Hilfsbereitschaft für jene Personen wichtig, die in der Überzeugung Partizipation höhere Werte aufweisen.

Betrachtet man die korrelativen Zusammenhänge der vier Überzeugungen mit den allgemeinen epistemologischen Überzeugungen, so zeigten sich erwartungskonforme Ergebnisse. Die Lehr-Lern-Überzeugung Transmission ging mit einem unkritischen Verständnis von Wissenschaft - Dualismus - einher. Die Konstruktion dagegen stand in Verbindung mit Bewusstsein, dass wissenschaftliche Theorien menschliche Konstruktionen darstellen. Jemand, der diese Lehr-Lern-Überzeugung aufweist, tendiert zu einem kritischen (relativistischen) Verständnis von Wissenschaft. Diese identifizierten Zusammenhänge bestätigten die Ergebnisse bisheriger Forschung (Chan \& Elliott, 2004; Seidel et al., 2006). Chan und Elliott zeigten Zusammenhänge der Transmission mit naiven epistemologischen Überzeugungen sowie der konstruktivistischen Überzeugung mit fortgeschrittenen Epistemologien. Ähnliches konnte in der Studie von Seidel et al. (2006) nachgewiesen werden, in welcher der Zusammenhang konstruktivistischer Lernüberzeugung mit einem Wissensverständnis als ein Netzwerk von sich ständig verändernden Theorien demonstriert wurde.

Zudem ergaben sich Hinweise auf einen Zusammenhang der Lehr-Lern-Überzeugung Partizipation mit fortgeschrittenen epistemologischen Überzeugungen. Der vorgefundene Zusammenhang der Partizipation mit dem Relativismus lässt sich vor dem theoretischen 
Hintergrund gut erklären. Einer kritischen, relativistischen Auffassung von Wissen entspricht die Einsicht in die Wissenschaft als das Ergebnis menschlicher Konstruktionsleistungen. Gerade für die Partizipation ist das Aushandeln des gemeinsamen Wissens in einer Lerngemeinschaft zentral (vgl. z.B. legitimate peripheral participation nach Lave \& Wenger, 1991; Sfard, 1998). Somit erscheint der positive Zusammenhang der Partizipation mit dem Relativismus theoriekonform und bestätigt formulierte Annahmen.

Weiterhin konnten in der vorliegenden Untersuchung sowohl geschlechts- als auch fächerkombinationsspezifische Unterschiede aufgezeigt werden. Im Einklang mit früheren Studien zeigten Studentinnen deskriptiv höhere Werte in der Überzeugung Partizipation als Studenten. So erzielten Studentinnen in der Studie von Collins et al. (2001) signifikant höhere Werte in den Perspektiven Schülerorientierung und Partizipation.

An dieser Stelle soll kritisch angemerkt werden, dass die Untergruppen in der vorliegenden Studie recht unterschiedlich ausfielen. Die Stichprobe setzte sich aus 48 männlichen und 133 weiblichen Probanden sowie 15 Studierenden der Naturwissenschaften, 116 der Geisteswissenschaften und 48 Studierenden mit der Fächerkombination aus beiden Fachgruppen zusammen, was sich enorm einschränkend auf die Testpower auswirkte.

Gleichwohl konnten in der vorliegenden Studie Unterschiede zwischen den Studierenden unterschiedlicher Fächerkombinationen gefunden werden. Verglichen mit den Studierenden der Gruppe Geisteswissenschaften und der gemischten Gruppe zeigten Studierende der Naturwissenschaften höhere Werte in der Überzeugung Konstruktion. Diese Ergebnisse fielen nicht im Einklang mit den Ergebnissen früherer Untersuchungen aus. In der Studie von Collins et al. (2001) bevorzugten Studierende der Mathematik und der Naturwissenschaften die Perspektive Transmission (siehe auch Norton et al., 2005), Studierende der Sozialwissenschaften hingegen die Perspektive Konstruktion (Collins et al., 2001). Diese Widersprüche zwischen den Ergebnissen der vorliegenden und früherer Studien können auf unterschiedliche Instrumente der Studien zurückgeführt werden und auch auf die Gruppenzuordnung der Studierenden in der vorliegenden Studie. Die meisten Studierenden (145 Bachelor- gegenüber 50 Masterstudierenden) befanden sich am Anfang ihres Studiums, sodass eine studienfachspezifische Sozialisation dieser zukünftigen Lehrkräfte zum Zeitpunkt der Befragung möglicherweise noch nicht ausreichend fortgeschritten war. Von daher konnten die erwarteten Unterschiede zwischen Studierenden unterschiedlicher Fächer nicht aufgezeigt werden.

In Übereinstimmung mit den formulierten Erwartungen zeigten Masterstudierende in der vorliegenden Studie verglichen mit Bachelorstudierenden höhere Werte in der 
Überzeugung Partizipation sowie niedrigere Werte in der Transmission. Es erscheint denkbar, dass die Inhalte entsprechender Lehr-/Lerntheorien in der Lehrerausbildung thematisiert wurden und die Studierenden immer mehr darüber erfuhren, sodass ihre Lehr-LernÜberzeugungen weniger transmissive und mehr partizipatorische Züge annahmen.

Insgesamt zeugen die durchgeführten Gruppenvergleiche von einer ausreichenden Sensitivität des Fragebogens gegenüber geschlechts- und fächerkombinationsspezifischen Unterschieden und Unterschieden in den Lehr-Lern-Überzeugungen in Abhängigkeit vom Zeitpunkt im Studium. Um allerdings weitere Aussagen treffen zu können - zum Beispiel darüber, welche Überzeugungen im Laufe des Studiums an Präferenz gewinnen - sollten Studien mit größeren Stichproben und in unterschiedlichen Populationen durchgeführt werden.

Zusammenfassend lässt sich festhalten, dass es in der vorliegenden Untersuchung gelungen ist, ein deutschsprachiges Instrument zur Erfassung der vier Lehr-LernÜberzeugungen Transmission, Konstruktion, Partizipation und Schülerorientierung zu entwickeln, das an die Forschung zu Lehrerüberzeugungen angebunden ist und gute psychometrische Eigenschaften aufweist. Mittels Faktoren-, Items- und Skalenanalysen konnten vier reliable Skalen sowie 14 Subskalen identifiziert werden, die die vier Überzeugungen zum Lehren und Lernen mit deren unterschiedlichen Facetten abbilden. Damit steht ein Fragebogen zur Verfügung, der eingesetzt werden kann, um beispielsweise die Beziehungen zwischen den Lehrerüberzeugungen zum Lehren und Lernen und den epistemologischen Überzeugungen zu untersuchen oder die Unterschiede in den Überzeugungen in Abhängigkeit von Unterrichtsfächern oder in Abhängigkeit vom Zeitpunkt in der Lehrerlaufbahn zu analysieren. Die mit dem Fragebogen ermittelten Ergebnisse können im Rahmen der Lehreraus- und -fortbildungsangebote verwendet werden.

\subsubsection{Ausblick}

Auf der Basis der Ergebnisse der vorliegenden Studie wurde der Q4TB leicht modifiziert. Diese Variante des Fragebogens wurde in einer weiteren Studie erneut eingesetzt. Das Instrument soll dabei weiteren Strukturprüfungen durch eine Faktorenanalyse unterzogen werden, um die Passung des Instruments an einer Studierendenstichprobe einer anderen Universität zu untersuchen. Somit kann der Fragebogen zur Klärung weiterer, im Lehr-LernKontext interessierender Fragen eingesetzt werden. Zum Beispiel wird er in der dritten Studie der vorliegenden Arbeit zur Untersuchung des Zusammenhangs zwischen epistemologischen 
Überzeugungen, erhoben mit dem Instrument nach Schommer (1990, 1992) und den vier lehr/lernbezogenen Überzeugungen Transmission, Konstruktion, Partizipation und Schülerorientierung verwendet. 


\section{6 Überprüfung des Zusammenhangs zwischen epistemologischen Überzeugungen und den lehr-/lernbezogenen Überzeugungen Transmission, Konstruktion, Partizipation und Schülerorientierung (Studie 3)}

\subsection{Zusammenfassung und Abstract}

\section{Zusammenfassung}

In der vorliegenden Studie wurde der Zusammenhang zwischen epistemologischen und lehr-/lernbezogenen Überzeugungen von Lehramtsstudierenden untersucht. Mithilfe des „Fragebogens zur Messung der vier Lehrerüberzeugungen zum Lehren und Lernen“ (The Questionnaire of Four Teachers' Beliefs - Q4TB) (Schlichter et al., in Vorbereitung) wurden Transmission, Konstruktion, Partizipation und Schülerorientierung an einer Stichprobe von $N$ $=110$ Studierenden erhoben. Epistemologische Überzeugungen wurden mithilfe vom „Epistemological Beliefs Questionnaire“ (EBQ) (Schommer, 1990) erhoben. Ermittelte Korrelationen zwischen epistemologischen und lehr-/lernbezogenen Überzeugungen bestätigten die Ergebnisse bisheriger Forschung: Während naive epistemologische Überzeugungen mit Transmission assoziiert wurden, gingen fortgeschrittene epistemologische Überzeugungen mit hohen Werten in Konstruktion einher. Ebenfalls wurde die theoretische Annahme eines fehlenden Zusammenhangs zwischen Schülerorientierung und epistemologischen Überzeugungen bestätigt. Entgegen den Erwartungen schien Partizipation nicht mit epistemologischen Überzeugungen assoziiert zu sein. Zudem deuteten die mit dem Verlauf des Studiums höheren Werte in Konstruktion und Partizipation auf eine Veränderung der lehr-/lernbezogenen Überzeugungen in die entsprechende Richtung. Dies bedeutet, dass konstrutivistische und partizipatorische Vorstellungen im Studium an Wichtigkeit gewinnen.

\section{Abstract}

The presented study explores the relationship between epistemological beliefs and teachers' beliefs about learning and teaching of student teachers in their teacher education. Using a sample of $N=110$ participants, teachers' beliefs transmission, construction, participation and nurturing were measured by means of the "Questionnaire of Four Teachers' Beliefs" (Q4TB) (Schlichter et al., in preparation). Epistemological beliefs were investigated by means of the EBQ (Schommer, 1990). Correlations between epistemological beliefs and beliefs about learning and teaching confirmed the results of the previous research: Naïve 
epistemological beliefs were associated with transmission and sophisticated epistemological beliefs with construction. The zero-correlation between epistemological beliefs and nurturing confirmed the theoretical assumption. Contrary, participation did not seem to be related to epistemological beliefs. Further, the revealed differences in construction and participation with the progress in studies indicated the development of teaches' beliefs toward these beliefs. That means that constructivist and participatory beliefs became important during the studies 


\subsection{Einleitung}

Unter dem Begriff „Überzeugung“ werden allgemein Annahmen über Charakteristika von Objekten (Nisbett \& Ross, 1980), mentale Repräsentationen gesammelter Erfahrungen (Sigel, 1985) oder gegebener Realität (Harvey, 1986) verstanden. Dabei umfasst der Oberbegriff „Lehrerüberzeugungen“ eine Vielfalt von Aspekten, über die sich Lehrkräfte subjektive Annahmen in Bezug auf ihren Beruf bilden können. Es kann sich um Überzeugungen $\mathrm{zu}$ Bildung, um Erwartungen bestimmter Schülerleistungen (Lehrerwirksamkeit) sowie um Überzeugungen $\mathrm{zu}$ den Ursachen von Lehrer- und Schülerleistung handeln (Attributionen). Unter epistemologischen Überzeugungen werden Ansichten über das Wissen, seine Struktur und Genese verstanden (Duell \& SchommerAikins, 2001; Hofer, 2000, 2001; Hofer \& Pintrich, 1997, 2002; Howard et al., 2000; Kitchener, 2002; Schommer, 1990; Sinatra, 2001). Epistemologische Überzeugungen von Lehrkräften sind somit ein Bereich aus dem Komplex der Lehrerüberzeugungen (Pajares, 1992).

In der Forschung wurde seit längerer Zeit die Annahme diskutiert, dass Lehrerüberzeugungen als intuitive Theorien die Art der Begegnung mit der erkennbaren Welt vorstrukturieren und nicht nur mit der Lehrerwahrnehmung vom Unterrichtsgeschehen und dem unterrichtlichen Handeln der Lehrkräfte, sondern auch mit den Lernprozessen der Schülerinnen und Schüler in Verbindung gebracht werden können (Hollingsworth, 1989; Nespor, 1987; Pajares, 1992). Dabei dürfte diese Annahme auf beide - epistemologische und lehr-/lernbezogene Überzeugungen von Lehrkräften als Teilbereiche der Lehrerüberzeugungen - zutreffen. Für diese Annahme wurde bereits empirische Evidenz geliefert (Peterson et al., 1989; Staub \& Stern, 2002). Zum Beispiel erzielten in der Studie von Staub und Stern (2002) diejenigen Schülerinnen und Schüler bessere Mathematikleistungen, deren Lehrkräfte konstruktivistische Überzeugungen zum Lernen besaßen. Auch stellten diese Lehrkräfte im Vergleich zu Lehrkräften mit transmissiven Überzeugungen anspruchsvollere Textaufgaben und boten den Schülerinnen und Schülern mehr Lerngelegenheiten zum tieferen Konzeptverständnis. In der COACTIV-Studie konnte gezeigt werden, dass je ausgeprägter die konstruktivistischen Überzeugungen von Lehrkräften waren, desto kognitiv aktivierender der Unterricht verlief und desto günstiger sich die Schülerleistungen in Mathematik entwickelten (Dubberke et al., 2008). Dies deutet darauf hin, dass Lehrerüberzeugungen, vermittelt über die Gestaltung von Lehr-/Lernprozessen, Einfluss auf das Lernen nehmen. 
Die Forschung zu epistemologischen Überzeugungen weist eine längere Tradition auf als die Forschung zu lehr-/lernbezogenen Lehrerüberzeugungen (Hofer \& Pintrich, 1997, 2002; Perry, 1970; Schommer, 1990). Allerdings wurde hier das Hauptaugenmerk auf die epistemologischen Überzeugungen von Schülerinnen und Schülern gerichtet und deren Einfluss zum Beispiel auf das Lernen von Schülern untersucht (Baumert et al., 2000; Trautwein \& Lüdtke, 2004). Im Vergleich dazu scheint das Interesse an epistemologischen Überzeugungen von Lehrkräften geringer zu sein (Hofer, 2001; Lampert, 1990; Maggioni \& Parkinson, 2008; Olafson \& Schraw, 2008). Gleichwohl wurden bereits empirische Hinweise dafür geliefert, dass epistemologische Überzeugungen von Lehrkräften eng mit ihrer Perspektive auf das Lernen von Schülerinnen und Schülern verbunden sind (Duschl, 1990; Ledermann, 1992) sowie das eigene Verständnis der Lehrerrolle und das unterrichtliche Handeln der Lehrkräfte beeinflussen (Etchberger \& Shaw, 1992; Martens, 1992). Bereits 1985 zeigte Hashweh, dass Lehrkräfte mit konstruktivistischen epistemologischen Überzeugungen ein breiteres Repertoire an Unterrichtsstrategien besaßen als ihre empirischorientierten Kolleginnen und Kollegen und zudem Unterrichtsstrategien verwendeten, die effektiver für den Konzeptwechsel der Schülerinnen und Schüler sein sollten.

Beide Lehrerüberzeugungen, epistemologische und lehr-/lernbezogene, wurden als Facetten professioneller Handlungskompetenz der Lehrkräfte definiert (vgl. Kunter et al., 2011). Allerdings wurden sie in der empirischen Forschung unterschiedlich operationalisiert. Zum einen wurden epistemologische Überzeugungen als Bereich von lehr-/lerntheoretischen Überzeugungen behandelt (Dubberke et al., 2008), zum anderen wurden ausschließlich lehrbezogene (Kember, 1997) oder lehr-/lernbezogene Überzeugungen (Peterson et al., 1989; Pratt et al., 2001; Staub \& Stern, 2001) untersucht. Die erste Art der Operationalisierung basiert scheinbar auf der Annahme, dass zum Beispiel die Vorstellung von Wissen als einfach und gesichert eine rezeptive Überzeugung zum Lernen mit sich bringt (Olafson \& Schraw, 2006). Dass diese Annahme nicht mit einem solchen Automatismus getroffen werden darf, zeigen die gegensätzlichen Befunde verschiedener Studien, die die Beziehung zwischen epistemologischen und lehr-/lernbezogenen Überzeugungen von Lehrkräften empirisch untersuchten. Dabei konnten einige Studien diese Annahme bestätigen (Chen \& Elliott, 2004; Seidel et al., 2008), andere lieferten gegensätzliche Hinweise. So demonstrierten Cheng et al. (2009) in ihrer Studie, dass fortgeschrittene epistemologische Überzeugungen mit traditionellen Lehr-Lern-Vorstellungen zusammenhängen können. Die Frage nach der Beziehung zwischen epistemologischen Überzeugungen und lehr-/lernbezogenen Überzeugungen scheint daher bislang nicht eindeutig beantwortet. Vor allem in Bezug auf die 
bisher wenig untersuchten partizipatorischen und schülerorientierten Lehr-LernÜberzeugungen besteht Forschungsbedarf. Hinsichtlich der lehr-/lernbezogenen Überzeugungen wurden in der aktuellen empirischen Forschung meistens transmissive und konstruktivistische Überzeugungen untersucht (Dubberke et al., 2008; Fennema et al., 1990; OECD; 2009; Staub \& Stern, 2002). Gleichwohl gibt es einige Studien, die sich auch mit partizipatorischen und schülerorientierten Überzeugungen befassen (Alger, 2009; Martinez et al., 2001). Da diese Studien qualitative Untersuchungen darstellen, war es bisher schwierig, die Bedeutung dieser Überzeugungen in Lehr-Lern-Kotexten zu untersuchen. Mit der Entwicklung des Q4TB (Studie 2: Schlichter, Nückles \& Watermann, in Vorbereitung) können Transmission, Konstruktion, aber auch Partizipation und Schülerorientierung analysiert und die Frage nach der Beziehung dieser lehr-/lernbezogenen Überzeugungen und der epistemologischen Überzeugungen untersucht werden. So wird in der vorliegenden Studie zum einen der Zusammenhang der transmissiven und konstruktivistischen Überzeugungen mit epistemologischen Überzeugungen überprüft. Zum anderen wird analysiert, inwieweit die lehr-/lernbezogenen Überzeugungen Partizipation und Schülerorientierung mit den epistemologischen Überzeugungen zusammenhängen. 


\subsection{Theoretischer Hintergrund}

\subsubsection{Lehr-/lernbezogene Überzeugungen von Lehrkräften}

Überzeugungen zum Lehren und Lernen sind implizite und explizite Annahmen über das Lehren - über den Lehrer und seine Rolle bzw. seine Aufgaben sowie über den Lernenden und seine Rolle bzw. seine Aufgaben. Lehrerüberzeugungen basieren auf Erfahrungen, sind subjektiv und bewertend und werden als Filter der Wahrnehmung (Kagan, 1992; Pajares, 1992; Weinstein, 1990) und als Handlungsdisposition verstanden (Brown \& Cooney, 1982; siehe auch Bandura, 1986; Dewey, 1933; Nisbett \& Ross, 1980; Rokeach, 1968).

Die aktuelle empirische Forschung konzentriert sich auf zwei Überzeugungen: Transmission und Konstruktion (Peterson et al., 1989; Staub \& Stern, 2002; Kunter et al., 2007). Eine transmissive Überzeugung zeichnet sich dadurch aus, dass Lehren als Übertragung von (strukturierten) Lerninhalten von der Lehrperson zu den Schülerinnen und Schülern (Sfard, 1998) im Sinne des Sender-Empfänger-Modells verstanden wird, während laut der konstruktivistischen Überzeugung Lernen ein aktiver Prozess ist, eigenes Wissen aufzubauen (Bereiter, 1994).

Die empirische Forschung kann bislang einige gut validierte Instrumente zur Messung von Transmission und Konstruktion vorweisen. Der Fragebogen von Peterson et al. (1998), welcher unter anderem in der Studie von Staub und Stern (2001) verwendet wurde, misst konstruktivistische Überzeugungen in Mathematik (Beispielitem: „Schüler lernen Mathematik am besten, indem sie selbst Wege zur Lösung von einfachen Textaufgaben entdecken“). Das in der COACTIV-Studie (Baumert et al., 2008) eingesetzte Instrument beinhaltet verschiedene Skalen zu transmissiven und konstruktivistischen Überzeugungen (Beispielitem: „Mathematik unterrichten heißt, Schüler/innen zum Nachdenken über mathematische Zusammenhänge zu bringen“). Auch in der internationalen OECD-Studie „TALIS“ (OECD, 2009) wurden diese beiden Überzeugungen untersucht. Andere Überzeugungen wurden nicht beachtet.

Gleichwohl gibt es Studien, die zwar keine großen Stichproben untersuchten und qualitative Methoden verwendeten, sodass generalisierende Aussagen schwierig erscheinen (Alger, 2009; Martínez et al., 2001). Gerade durch ihre Methode lieferten diese Studien aber Hinweise auf neue, wenig erforschte Ansichten zum Lehren und Lernen, nämlich auf partizipatorische und schülerorientierte Überzeugungen zum Lehren und Lernen. In der partizipatorischen Sicht wird der Fokus auf die Lerngruppe gerichtet und das Lernen als 
Enkulturation eines neuen Mitglieds in die Lerngemeinschaft durch die Aneignung und den Aufbau kollektiv geteilten Wissens betrachtet (Lave \& Wenger, 1991). Bei der Schülerorientierung geht es um die Schülerpersönlichkeit sowie um die Lehrer-SchülerBeziehung (Alger, 2009; Pratt, 2005). Hier bedeutet Lernen persönliches Wachstum und Entwicklung individuell angelegter Fähigkeiten in einer schülerorientierten Lernatmosphäre (Klieme et al., 2006), welche etwa durch Beachtung der Bedürfnisse nach Autonomie, Kompetenz und sozialer Eingebundenheit (Deci \& Ryan, 1993) gesichert werden kann.

Die vier verschiedenen Überzeugungen lassen sich auf unterschiedliche theoretische Paradigmen zurückzuführen: 1) die Überzeugung Transmission auf die behavioristische LehrLern-Theorie, 2) die Überzeugung Konstruktion auf die konstruktivistische Lerntheorie, 3) die Überzeugung Partizipation auf den Lerngemeinschaftsansatz (Lave \& Wenger, 1991) und den Apprenticeship-Lernansatz (Collins et al., 1989; Palincsar \& Brown, 1984; Scardamalia \& Bereiter, 1987) sowie 4) die Überzeugung Schülerorientierung auf das Konstrukt der Schülerorientierung nach Klieme et al. (2006) und auf die humanistische Lerntheorie (Huitt, 2009).

Während die empirische Forschung eine Reihe gut validierter Instrumente für die Erfassung von Transmission und Konstruktion vorgelegt hat, gab es nach dem heutigen Wissensstand bislang lediglich einen englischsprachigen Fragebogen, das TPI, mit dessen Hilfe verschiedene, auch partizipatorische und schülerorientierte Perspektiven untersucht wurden (Pratt et al., 2001). Allerdings wurden für das TPI bislang keine entsprechenden Validierungsstudien publiziert und auch Validierungsversuche einer deutschen Übersetzung des Fragebogens verliefen wenig erfolgreich (vgl. Hanke, 2011). Der deutschsprachige Fragebogen zur Messung der vier Lehrerüberzeugungen zum Lehren und Lernen Q4TB stellt ein Instrument dar, das neben Transmission und Konstruktion auch Partizipation und Schülerorientierung misst. Dieser Fragebogen weist gute psychometrische Eigenschaften auf und erlaubt eine reliable und valide Erfassung der vier Lehrerüberzeugungen. Zudem wurde der Fragebogen bereits validiert (Studie 2: Schlichter et al., in Vorbereitung). 


\subsection{2 Überblick über die Forschung zur Veränderung von lehr-/lernbezogenen Überzeugungen}

Die Frage nach der Veränderung der Lehrerüberzeugungen wird in der Literatur kontrovers diskutiert. Einerseits wird stets die Änderungsresistenz der Überzeugungen betont (Kagan, 1992; Pajares, 1992; Weinstein, 1990). Andererseits erscheint die Annahme der Entwicklung von Lehrerüberzeugungen plausibel. Lehrkräfte erwerben neue Kenntnisse im Verlauf ihrer beruflichen Karriere, in der Lehreraus- und -weiterbildung, und sammeln zahlreiche Erfahrungen in verschiedenen Lehr-Lern-Kontexten, sodass diese neuen Kenntnisse und Erfahrungen auf die Lehrerüberzeugungen einwirken, da Überzeugungen laut Definition auf gesammelten Erfahrungen basieren (u. a. Sigel, 1985). Gleichwohl wurde diese theoretische Annahme der Veränderung von Lehrerüberzeugung empirisch nur wenig erforscht (z.B. Calderhead, 1996; Wan Ng et al., 2010; Russel, 1988; Richardson, 1996). Die wenigen, meist qualitativen Studien lieferten Hinweise auf eine Veränderung der Lehrerüberzeugungen im Verlauf der Lehrerausbildung (Saban, 2010; Saban et al., 2007). So wurden Überzeugungen von Studierenden am Anfang ihrer Lehrerausbildung als transmissiv und emotionsorientiert beschrieben (Nettle, 1998). Sie sollten sich jedoch im Verlauf des Studiums von dieser Emotions- zu einer Aufgabenorientierung (Nettle, 1998) und von Schülern-Helfen zum Klassen-Managen (Rust, 1994) verändern. Dieses Ergebnis wurde in der Interview-Studie der vorliegenden Arbeit bestätigt (Studie 1): Die Überzeugung Schülerorientierung, in welcher das "Schülern-Helfen“ erkennbar ist, war am Anfang des Studiums wichtig, verlor jedoch bereits in der Mitte des Studiums an Bedeutung und auch gegen Ende des Studiums fand sie keine Beliebtheit mehr (Studie 1: Schlichter et al., in Vorbereitung). Insgesamt scheinen sich die Überzeugungen zukünftiger Lehrkräfte im Verlauf der Lehrerausbildung in Richtung Konstruktivismus zu verändern (Hollingsworth, 1989; Leavy et al., 2007), was auch die Interviewstudie der vorliegenden Arbeit zeigte (Studie 1: Schlichter et al., in Vorbereitung).

Gleichwohl werden die Ergebnisse bisheriger Forschung, vor allem im Hinblick auf partizipatorische und schülerorientierte Überzeugungen, als nicht ausreichend betrachtet. Die Interviewstudie der vorliegenden Arbeit (Studie 1: Schlichter et al., in Vorbereitung) stellte den ersten Versuch dar, partizipatorische und schülerorientierte Überzeugungen zu unterschiedlichen Zeitpunkten in der Lehrerausbildung zu untersuchen. Hinsichtlich der Partizipation zeigten sich am Anfang des Studiums niedrige Werte, die bereits zur Mitte des Studiums anstiegen und bis zum Ende des Studiums auf einem hohen Niveau blieben. Für die 
Schülerorientierung ergab sich eine gegensätzliche Entwicklung, die aber keinen signifikanten Unterschied zwischen den drei Zeitpunkten in der Lehrerausbildung identifizieren konnte.

Insgesamt lassen die berichteten Ergebnisse eine Veränderung von lehr/lernbezogenen Überzeugungen annehmen, welche auf die Lehrerausbildung zurückgeführt werden kann. Allerdings muss darauf hingewiesen werden, dass die genannten Studien qualitative Ansätze verwendeten. Einige davon (Saban, 2010; Saban et al., 2007) untersuchten Lehrerüberzeugungen, indem sie Metaphern des Lehrens und Lernens analysierten. Die Ergebnisse dieser Studien können demnach nur mit Vorsicht interpretiert werden. Eine Untersuchung mit quantitativen Verfahren und größeren Stichproben wird somit als vorteilhaft erachtet. Die Ergebnisse solcher Untersuchungen können mit relativ wenig Aufwand mittels einer wiederholten Messung überprüft werden. In diesem Zusammenhang stellt das neu entwickelte und in einer Studie (Studie 2: Schlichter et al., in Vorbereitung) validierte Q4TB eine Möglichkeit dar, Transmission, Konstruktion, aber auch Partizipation und Schülerorientierung zu unterschiedlichen Zeitpunkten in der Lehrerausbildung und Lehrerlaufbahn zu untersuchen. Die Ergebnisse der Validierungsstudie lieferten bereits erste Hinweise darauf, dass sich die lehr-/lernbezogenen Überzeugungen von Bachelor- und Masterstudierenden unterscheiden. Während Bachelorstudierende höhere Werte in Transmission zeigten, schienen Masterstudierende mehr partizipatorische Einsichten in ihren lehr-/lernbezogenen Überzeugungen aufzuweisen. Daher wurde für die vorliegende Untersuchung der Fragebogen Q4TB ausgewählt, um die lehr-/lernbezogenen Überzeugungen Transmission, Konstruktion, Partizipation und Schülerorientierung zu erheben und der zentralen Frage nach der Beziehung epistemologischer Überzeugungen und der vier genannten lehr-/lernbezogenen Überzeugungen nachgehen zu können sowie die vier lehr/lernbezogenen Überzeugungen in Abhängigkeit vom Zeitpunkt in der Lehrerausbildung zu analysieren.

\subsubsection{Epistemologische Überzeugungen}

Epistemologische Überzeugungen werden als Ansichten über das Wissen, seine Genese und Struktur (Duell \& Schommer-Aikins, 2001; Hofer, 2000, 2001; Hofer \& Pintrich, 1997; Schommer, 1990) verstanden. Epistemologischen Überzeugungen wurde in der Literatur große Aufmerksamkeit geschenkt, wobei es sich hauptsächlich um Überzeugungen von Schülerinnen und Schülern sowie Studierenden handelte (Hofer \& Pintrich, 1997, 2002; Kitchener, 2002; Sinatra, 2001; Kuhn, 1999; Pillow, 1999). Es existieren viele Modelle 
epistemologischer Überzeugungen. Dabei beschreiben Entwicklungsmodelle verschiedene Stufen epistemologischer Überzeugungen und nehmen eine Entwicklung entlang dieser Stufen an, die mit dem Alter und dem Bildungsstand voranschreitet. Ein dualistisches Wissensverständnis mit der Auffassung vom Wissen als richtig oder falsch (Perry, 1970; Trautwein \& Lüdtke, 2004) wird von einem multiplistischen Wissensverständnis (Perry, 1970), das verschiedene Standpunkte anerkennt, abgelöst. Eine weitere Stufe bildet dann die relativistische Auffassung von Wissen mit der Einsicht in die Subjektivität wissenschaftlicher Erkenntnis (Perry, 1970) und der kritischen Einstellung gegenüber wissenschaftlichen Theorien (Trautwein \& Lüdtke, 2004). In der Forschung zu epistemologischen Überzeugungen wurden viele Untersuchungen mit Schülerinnen und Schülern sowie Studierenden durchgeführt. Gerade diese Unterscheidung einer dualistischen und relativistischen epistemologischen Ansicht wurde in der Studie „Transformation des Sekundärschulsystems und akademische Karrieren“ (TOSCA: z.B. Köller et al., 2004) mit deutschen Jugendlichen vorgenommen.

Auch bei epistemologischen Einsichten von Lehrkräften wurde oft prototypisch zwischen verschiedenen Epistemologien unterschieden und die Lehrkräfte wurden einem Typ mit einer bestimmten epistemologischen Sicht zugeordnet (Levitt, 2001; Olafson \& Schraw, 2002; White, 2000; Wilcox-Herzog, 2002). So sprechen sich einige Autoren dafür aus, dass Lehrkräfte über eine holistische Auffassung von Wissen verfügen (Lehrer, 1990; Pollock, 1986). Olafson und Schraw (2006) beschreiben drei solcher holistischen epistemologischen Weltbilder: a) die realistische Weltsicht mit der Auffassung, dass sich das Wissen aus fest definierten, fertigen Bestandteilen zusammensetzt, b) die relativistische Weltsicht mit dem Glauben, dass jeder sein eigener Wissenskonstrukteur ist und dass sich das Wissen ständig verändert, sowie c) die kontextualistische Weltsicht mit der Vorstellung, dass Schülerinnen und Schüler lernen, indem sie mit Lehrerunterstützung ein gemeinsames Verständnis von Wissen konzipieren (Olafson \& Schraw, 2002, 2008).

In den Studien von Hashweh (1985, 1996), Tsai (2006) und Seidel et al. (2008), die sich mit epistemologischen Überzeugungen von Lehrkräften befassen, findet sich die Unterscheidung in konstruktivistische und empiristische epistemologische Überzeugungen. Bei einem konstruktivistischen Wissensverständnis werden die Vorläufigkeit von Wissen, die theoretisch begründete Qualität der wissenschaftlichen Exploration und die Rolle des Konzeptwechsels in der fortschreitenden Entwicklung wissenschaftlichen Verständnisses betont (Seidel et al., 2008). Kennzeichnend ist zudem die Annahme, dass Wissenschaft auf sozialen Aushandlungsprozessen und Vereinbarungen beruht, die zum Beispiel Paradigmen 
und akzeptierte Beweisführungen betreffen. Charakteristisch für eine empiristische Überzeugung ist demgegenüber das Verständnis, dass Wissenschaft eine objektive Realität aufdeckt, die unabhängig von individuellen Sichtweisen existiert (Tsai, 2006).

In der pädagogischen Psychologie konnte sich das aus fünf unabhängigen Dimensionen des Wissens bestehende Modell von Schommer (1994b) durchsetzen. Dieses Modell unterscheidet a) die Struktur des Wissens, b) die Sicherheit und c) die Quelle des Wissens sowie d) die Lernfähigkeit und e) die Lerngeschwindigkeit (Schommer, 1998). Trotz der Kritik an diesem Modell, zum Beispiel bezüglich der fächerübergreifenden Betrachtung der Überzeugungen, kann der SEQ nach Schommer (1990, 1993) als ein gut validiertes Instrument betrachtet werden, das in einer Reihe von Studien mit Schülerinnen und Schülern sowie mit Studierenden überprüft werden konnte. Mit diesem Instrument kann zwischen naiven und elaborierten epistemologischen Überzeugungen unterschieden werden. Einer naiven Überzeugung entspricht die Vorstellung von Wissen als einfach, klar, sicher und genau bestimmt sowie der Glaube an eine angeborene unveränderbare Lernfähigkeit und daran, dass man entweder etwas schnell lernt oder gar nicht lernen kann. Im Gegensatz dazu ist eine fortgeschrittene epistemologische Überzeugung die Vorstellung von Wissen als komplex, unsicher, vorläufig gültig, schrittweise erlern- und erfahrbar, vom einzelnen Lerner konstruierbar und in Diskursen verhandelbar. Chen und Elliott (2004) verwendeten das Instrument von Schommer (1990) in ihrer Studie mit Lehrkräften und konnten folgende vier Faktoren bestätigen: angeborene Lernfähigkeit, Lernanstrengung, Wissen der Experten und sicheres Wissen.

Demnach lässt sich schlussfolgern, dass in der Lehrerforschung viele verschiedene Modelle epistemologischer Überzeugungen Einsatz fanden. Gleichwohl lässt sich bei der Betrachtung verschiedener Studien erkennen, dass diese entweder eine holistische Betrachtung epistemologischer Überzeugungen bevorzugen, indem sie Lehrkräfte einer der zwei oder drei verschiedenen Typen zuordnen - realistische vs. relativistische vs. kontextualistische Weltsicht (Olafson \& Schraw, 2002, 2006, 2008) - oder anhand der Skalen beschreiben - konstruktivistische vs. empiristische Epistemologien (Seidel et al., 2006) - oder dualistische vs. relativistische (Trautwein \& Lüdtke, 2004) oder naive vs. fortgeschrittene Überzeugungen mithilfe der verschiedenen Dimensionen nach Schommer darlegen (Chen \& Elliott, 2004). Zwar befassen sie sich mit ähnlichen Konstrukten epistemologischer Überzeugungen, ihre Ergebnisse lassen sich jedoch aufgrund von begrifflichen Unterschieden nur schwer in Verbindung miteinander bringen. 
Weiterhin ist zur Forschung hinsichtlich epistemologischer Überzeugungen festzuhalten, dass einige gut validierte Instrumente zur Erfassung epistemologischer Überzeugungen existieren. Besonders hervorzuheben ist das Instrument aus der Studie „Transformation des Sekundärschulsystems und akademische Karrieren“ (TOSCA) (Schiefele, Moschner \& Husstegge, 2002; Trautwein \& Lüdtke, 2004) oder die deutsche Übersetzung des SEQ (Schommer, 1993) in Überarbeitung von Gruber und Renkl (1995) (Salhab, 2003). In der empirischen Forschung hat sich der SEQ (Schommer, 1990, 1993) durchgesetzt. Trotz Kritik wurde er in vielen Studien eingesetzt. Zudem liegt eine evaluierte deutsche Version des Fragebogens vor (Gruber \& Renkl, 1995). Das Instrument von Schommer stellt außerdem eine Möglichkeit dar, neben den allgemeinen Dimensionen wie der Struktur des Wissens auch lernspezifische epistemologische Überzeugungen (Lernfähigkeit und Lerngeschwindigkeit) zu erheben. Daher wurde dieses Instrument für die vorliegende Untersuchung ausgewählt, um die Frage nach der Beziehung zwischen lehr-/lernbezogenen Überzeugungen und epistemologischen Überzeugungen zu analysieren. 


\subsubsection{Diskrepanzen in der Definition und Operationalisierung von epistemologischen und lehr-/lernbezogenen Lehrerüberzeugungen}

Epistemologische Überzeugungen und lehr-/lerntheoretische Überzeugungen werden in der Lehrerforschung als in Verbindung mit der Qualität der Lehr-Lern-Prozesse stehende Konstrukte diskutiert. Dennoch lassen sich in der Operationalisierung der Konstrukte große Diskrepanzen feststellen (Dubberke et al., 2008; Chan \& Elliott, 2004; Seidel et al., 2008). Während beide als Facetten professioneller Handlungskompetenz von Lehrkräften aufgefasst werden (Kunter et al., 2011), werden sie jedoch nicht einheitlich definiert und operationalisiert (Dubberke et al., 2008). In einigen Studien erfolgt die Behandlung epistemologischer Überzeugungen als Teilbereich der lehr-/lernbezogenen Überzeugungen (Aguirre \& Speer, 2000; Dubberke et al., 2008), in anderen werden sie bei Untersuchungen von Lehr-Lern-Überzeugungen gänzlich ausgeklammert (Kember, 1997; Peterson et al., 1989; Staub \& Stern, 2002). Obwohl beide Konstrukte Lehrerüberzeugungen beschreiben, befinden sie sich konzeptuell auf unterschiedlichen Ebenen - mit Pajares Worten (1992) liegen die einen eher im Zentrum und die anderen verstärkt an der Peripherie der Überzeugungssysteme. Epistemologische Überzeugungen geben zum Beispiel den Glauben einer Lehrkraft an die Begabung eines Schülers wieder oder die Ansicht, dass ein Schüler einen Sachverhalt „nie lernt, wenn er [ihn] nicht beim ersten Mal verstanden hat" (ein Item aus dem SEQ nach Schommer, 1990, 1993). Dagegen lassen Überzeugungen zum Lehren und Lernen eine Lehrkraft den Vorgang des Lernens als zum Beispiel eine eigene Wissenskonstruktionsleistung von Schülerinnen und Schülern auffassen.

In der Tradition der Untersuchungen von fachspezifisch-pädagogischem Wissen der Lehrkräfte und deren Überzeugungen (Chi et al., 1988; Peterson et al., 1989; Shulman, 1986, 1987; Sternberg \& Horvath, 1999) wurden viele Studien im Fach Mathematik durchgeführt (Aguirre \& Speer, 2000; Hashweh, 1985, 1996; Stipek et al., 2001). Dabei wurden Lehrerüberzeugungen als Überzeugungen über das Wesen von Mathematik und auch Überzeugungen über Lehren und Lernen verstanden (Ernest, 1989; Thompson, 1984, 1985, 1992). In der deutschsprachigen Forschung lässt sich diese Tendenz zur Aufnahme beider Überzeugungen - epistemologischer und lehr-/lernbezogener - in das Konstrukt der Lehrerüberzeugungen ebenfalls erkennen (Clausen et al., 2003; Dubberke et al., 2008; Lipowsky et al., 2003). So wurden in der COACTIV-Studie lerntheoretische Überzeugungen der Lehrkräfte mit einer Skala zu epistemologischen Überzeugungen nach Grigutsch et al. (1996) - „Mathematik als Toolbox“ - sowie Skalen nach Fennema et al. (1990) zu 
Mathematiklernen - „Rezeptives Lernen durch Beispiele und Vormachen“, „Eindeutigkeit des Lösungsweges“ und „Einschleifen von technischem Wissen“ - erhoben (Dubberke et al., 2008). Ebenfalls beide Überzeugungen wurden in der deutsch-schweizerischen Vergleichsstudie von Lipowsky et al. (2003) in die Analysen der Lehrerüberzeugungen im Fach Mathematik einbezogen: die Philosophie des Schulfaches (Skala nach Grigutsch et al., 1996) und Skalen zum fachspezifischen pädagogischen Wissen nach Fennema et al. (1990) in der deutschen Adaption von Staub und Stern (2002).

Hinsichtlich der Überzeugungen von Lehrkräften im universitären Bereich wurden ausschließlich lehr-/lernbezogene Überzeugungen untersucht, ohne Beachtung epistemologischer Einsichten. Dabei wurden mehrere Überzeugungen unterschieden: Vermittlung von Wissen, Förderung von Lernprozessen (Gow \& Kember, 1993; Kember, 1997) sowie lehrer-orientierte und schüler-orientiere Lehrkonzeptionen (Norton et al., 2005; Trigwell \& Prosser, 2004) oder unter anderem transmissive, konstruktivistische und schülerorientierte Lehrperspektiven (Pratt et al., 2001). Aber nicht nur im universitären Bereich wurden bei der Untersuchung von Lehrerüberzeugungen epistemologische Überzeugungen ausgeklammert. So wurden in den Studien mit Mathematiklehrkräften von Staub und Stern (2002) und bei Peterson et al. (1989) aus dem Schulkontext keine Überzeugungen über das Fach, sondern ausschließlich lehr-lern-theoretische Überzeugungen mit folgenden Skalen erhoben: Rolle des Lerners, Relation zwischen Fähigkeiten, Verständnis und Problemlösen, Sozialkonstruktivismus und Rolle des Lehrers. Auch Hartinger et al. (2006) beschäftigten sich mit allgemeinen, also bereichsunspezifischen Lehr-LernVorstellungen von Grundschullehrkräften, ohne epistemologische Überzeugungen in die Analysen aufzunehmen.

Zusammenfassend lässt sich festhalten, dass die Operationalisierung der beiden Konstrukte große Diskrepanzen erkennen lässt. Zum Teil wurden die Begriffe gemeinsam als Lehrerüberzeugungen erfasst, zum Teil wurden epistemologische Überzeugungen ausgeklammert. Daher wird für die zukünftige Forschung die Trennung zwischen beiden Begriffen für sinnvoll erachtet. 


\subsection{5 Überblick über die Forschung zum Zusammenhang zwischen epistemologischen Überzeugungen und lehr-/lernbezogenen Überzeugungen}

Theoretisch wurde der Zusammenhang zwischen epistemologischen Überzeugungen und lehr-/lernbezogenen Überzeugungen oft thematisiert (Clark \& Peterson, 1986; Fennema \& Loef Franke, 1992; Marland, 1995; 1998; Pajares, 1992; Richardson, 1996). Allgemein wird angenommen, dass Lehrkräfte, die Wissen als Ansammlung absoluter Wahrheiten verstehen, ihre Rolle in der Vermittlung von Wissensgrundlagen und das Ziel des Lernens im Beherrschen von Fakten sehen (Millar, 1989). Lehrkräfte, die das Wissen als ein Netzwerk von Theorien verstehen, sind hingegen der Überzeugung, dass Schülerinnen und Schüler ihr Wissen durch Verständigungs- und Aushandlungsprozesse selbst konstruieren (Olafson \& Schraw, 2006).

Studien, die unter Lehrerüberzeugungen sowohl epistemologische als auch lehr-/lernbezogene Überzeugungen auffassen, weisen eine intergrative Betrachtungen von epistemologischen Überzeugungen und Überzeugungen zum Lernen und Lehren auf (Handal, 2003). In der COACTIV-Studie zum Beispiel wird von „Überzeugungssyndromen“ gesprochen und davon ausgegangen, dass epistemologische Überzeugungen und Überzeugungen zum Lernen und Lehren in charakteristischen Mustern zusammenfallen, die sich entweder stärker an konstruktivistischen oder stärker an transmissiv/behavioristischen Lerntheorien orientieren (Voss, Kleickmann, Kunter \& Hachfeld, 2011). Daher bleibt die Annahme, dass eine bestimmte Vorstellung von Wissen eine bestimmte Vorstellung von Lernen mit sich bringt, nict hinterfragt. Dieses fehlende Hinterfragen kann allerdings kritisch betrachtet werden, zumal es mittlerweile Hinweise darauf gibt, dass diese Annahme nicht immer gilt und dass auch fortgeschrittene epistemologische Überzeugungen mit traditionellen Lehr-Lern-Vorstellungen zusammenhängen können (Cheng, Chan, Tang \& Cheng, 2009).

Die empirische Forschung lieferte Hinweise darauf, dass epistemologische Überzeugungen eng mit der Sicht von Schülerinnen und Schülern auf das Lernen verbunden sind (Duschl, 1990; Ledermann, 1992) und die Auswahl von Lehrmethoden steuern (Hashweh, 1996). Die Ergebnisse aktueller Studien (Chan \& Elliott, 2004; Cheng et al., 2009) zeigten, dass epistemologische Überzeugungen die Art der Überzeugungen zum Lehren und Lernen beeinflussen. In der Studie von Chan und Elliott (2004) wurden Daten von 385 Lehramtsstudierenden aus Hong Kong hinsichtlich ihrer lehr-/lernbezogenen und ihrer epistemologischen Überzeugungen erhoben. Bei den Lehr-Lern-Überzeugungen unterschieden die Autoren in Anlehnung an Dunkin und Precians (1992) zwischen traditionellen und 
konstruktivistischen Lehr-Lern-Konzeptionen, und bei den epistemologischen Überzeugungen orientierten sie sich am SEQ (Schommer, 1990, 1993) mit den vier Faktoren angeborene Lernfähigkeit, Lernanstrengung, Wissen der Experten und sicheres Wissen. Die Ergebnisse zeigten einen positiven Zusammenhang zwischen einer traditionellen Vorstellung von Lehren und Lernen und dem Glauben an eine angeborene Lernfähigkeit $(r=.40, p<$ $.001)$, dem Wissen der Experten $(r=.40, p<.001)$ und sicherem Wissen $(r=.31, p<.001)$. Zudem wurde ein negativer Zusammenhang einer konstruktivistischen Vorstellung vom Lehren und Lernen und der angeborenen Lernfähigkeit $(r=-.16, p<.01)$ und dem Wissen des Experten $(r=-.22, p<.001)$ sowie ein positiver Zusammenhang mit Lernanstrengung $(r=$ $.39, p<.001)$ nachgewiesen. Des Weiteren wurde die Annahme eines kausalen Zusammenhangs zwischen epistemologischen Überzeugungen und Überzeugungen zum Lehren und Lernen mithilfe von Strukturgleichungsmodellen überprüft. Das Ergebnis zeigte einen signifikanten Effekt des Glaubens an eine angeborene Lernfähigkeit $(\beta=.52, t=6.45, p$ $<.05)$, Wissen des Experten $(\beta=61, t=5.88, p<.05)$ und sicheres Wissen $(\beta=.46, t=5.66$, $p<.05)$ auf die traditionelle Vorstellung von Lehren und Lernen sowie des Glaubens an Lernanstrengung $(\beta=-.56, t=-7.01, p<.05)$ auf die konstruktivistische Vorstellung von Lehren und Lernen.

In einer weiteren Studie mit 50 deutschen Physiklehrkräften wurden lehr/lerntheoretische Überzeugungen (Fennema et al., 1990) und epistemologische Überzeugungen (Köller et al., 2000) sowie deren Beziehung zueinander untersucht (Seidel et al., 2008). Es wurde die Vermutung überprüft, dass die Vorstellung von Wissenschaft als eine objektive, von individuellen Sichtweisen unabhängige Realität (empiristisches Wissensverständnis) mit einer rezeptiven Überzeugung zum Lernen zusammenhängt. Dagegen sollen Lehrkräfte mit einem Verständnis von Wissen als einem Netzwerk von Theorien, das sich mit neuen Erkenntnissen ständig verändert (konstruktivistisches Wissensverständnis), ihre Aufgabe in der Bereitstellung von Lernumgebungen sehen, die Lernende in den Prozess des Wissensaufbaus und der Generierung von Wissen einbinden. Die Ergebnisse der Studie von Seidel et al. (2008) bestätigten diese Annahme, da ein positiver Zusammenhang des konstruktivistischen Wissensverständnisses mit der konstruktivistischen Überzeugung zum Schülerlernen $(r=.41, p<.005)$ sowie ein positiver Zusammenhang eines empiristischen Wissensverständnisses mit einer rezeptiven Überzeugung zum Schülerlernen $(r=.28, p<.05)$ festgestellt wurden.

In der Studie von Cheng et al. (2009) wurde die Beziehung zwischen epistemologischen Überzeugungen und Konzeptionen zum Lehren und Lernen mithilfe 
quantitativer und qualitativer Methoden überprüft. Im Einklang mit der Studie von Chan und Elliott (2004) wurde bei 12 zukünftigen Lehrkräften mit fortgeschrittenen epistemologischen Vorstellungen eine konstruktivistische Vorstellung von Lehren und Lernen vorgefunden. Allerdings wurde auch bei vier Studierenden mit fortgeschrittenen epistemologischen Vorstellungen eine Kombination aus konstruktivistischen und traditionellen Vorstellungen von Lehren und Lernen identifiziert (Cheng et al., 2009, S. 324).

Zusammenfassend lässt sich festhalten, dass von einem Zusammenhang zwischen naiven (empiristischen) epistemologischen Überzeugungen und der lehr-/lernbezogenen Überzeugung Transmission sowie von einem Zusammenhang zwischen fortgeschrittenen (konstruktivistischen) epistemologischen Überzeugungen und der lehr-/lernbezogenen Überzeugung Konstruktion ausgegangen werden kann.

Da die bisherige Forschung ausschließlich die konstruktivistische und traditionelle Überzeugung zum Lehren und Lernen untersucht hat, wurde bisher die Beziehung zwischen den lehr-/lernbezogenen Überzeugungen Partizipation und Schülerorientierung und epistemologischen Überzeugungen nicht untersucht. In diesem Zusammenhang interessiert daher die Frage, ob und in welcher Beziehung Partizipation und Schülerorientierung zu epistemologischen Überzeugungen stehen. Entsprechend der Lerntheorie des Lerngemeinschaftsansatzes (Lave \& Wenger, 1991) wird das Wissen nicht von einer Einzelperson konstruiert, sondern im Prozess authentischer Kommunikation von den Mitgliedern der Lerngemeinschaft ausgehandelt, sodass ein gemeinsames Wissen entsteht (Sfard, 1998; Lave \& Wenger, 1991). Diese Lerntheorie stellt die theoretische Basis der lehr/lernbezogenen Überzeugung Partizipation dar. Daher wäre eine Beziehung zwischen der Partizipation und epistemologischen Überzeugungen denkbar. Da Wissensaushandlungsprozesse im Lerngemeinschaftsansatz von Bedeutung sind, könnte dementsprechend eine Verbindung zwischen der Überzeugung Partizipation und fortgeschrittenen Überzeugungen vermutet werden, die ähnlich wie der Lerngemeinschaftsansatz von einer Konstruktion des Wissens ausgeht. Dagegen dürften die Vorstellungen von Wissen für die lehr-/lernbezogene Überzeugung Schülerorientierung von geringer Bedeutung sein. Bei der Schülerorientierung geht es in erster Linie um die Schülerpersönlichkeit mit ihren Bedürfnissen nach Autonomie, Kompetenzerleben und sozialer Eingebundenheit. Das bedeutet, dass eher motivationale Aspekte und weniger kognitive Aspekte des Lernens beachtet werden. Daher wäre hier eine Unabhängigkeit der Konstrukte denkbar. 
In der vorliegenden Studie wird der Frage nach dem Zusammenhang zwischen den lehr-/lernbezogenen Überzeugungen Transmission, Konstruktion, Partizipation und Schülerorientierung und den naiven bzw. fortgeschrittenen epistemologischen Überzeugungen nachgegangen. 


\subsection{Ableitung der Fragestellung}

In der vorliegenden Untersuchung soll der Zusammenhang zwischen epistemologischen und lehr-/lernbezogenen Überzeugungen analysiert werden. Die zentrale Fragestellung der vorliegenden Arbeit lautet wie folgt:

1) Gibt es einen Zusammenhang zwischen den naiven und fortgeschrittenen epistemologischen und den lehr-/lernbezogenen Überzeugungen Transmission, Konstruktion, Partizipation und Schülerorientierung?

Vor dem Hintergrund aktueller Forschung wird ein Zusammenhang zwischen Transmission und naiven epistemologischen Überzeugungen sowie zwischen Konstruktion und fortgeschrittenen epistemologischen Überzeugungen erwartet. Bei Partizipation und Schülerorientierung können Hypothesen ausschließlich auf der Basis theoretischer Grundlagen formuliert werden, da zu diesen beiden Überzeugungen keine Untersuchungen vorliegen. Es wird erwartet, dass die Partizipation mit fortgeschrittenen epistemologischen Überzeugungen zusammenhängt, da es sich bei dem Lerngemeinschaftsansatz, welcher der Partizipation zugrunde liegt, um eine gesellschaftliche Konstruktion von Wissen handelt. Für die lehr-/lernbezogene Überzeugung Schülerorientierung wird dagegen vermutet, dass diese Überzeugung nicht mit dem Konstrukt epistemologischer Überzeugungen zusammenhängt. Bei der Schülerorientierung handelt es sich um die Schülerpersönlichkeit mit ihren individuellen Bedürfnissen, sodass die Ansichten über das Wissen hier weniger wichtig sind.

In dieser Studie wird zudem der Frage nachgegangen, ob sich die lehr-/lernbezogenen Überzeugungen in Abhängigkeit vom Zeitpunkt im Studium unterscheiden. Es wird untersucht, inwieweit Unterschiede in den lehr-/lernbezogenen Überzeugungen zwischen Studierenden am Anfang, in der Mitte und am Ende des Studiums vorliegen.

2) Unterscheiden sich die lehr-/lernbezogenen Überzeugungen in Abhängigkeit vom Zeitpunkt im Studium?

Vor dem Hintergrund bisheriger Forschung sowie der Ergebnisse der Studien 1 und 2 können Unterschiede in den lehr-/lernbezogenen Überzeugungen von Studierenden am Anfang, in der Mitte und am Ende des Studiums erwartet werden. Aufgrund der im Studium vermittelten lehr-/lerntheoretischen Kenntnisse, wie zum Beispiel zum Konstruktivismus, 
Kapitel 6

wird eine Präferenz fortgeschrittener Studierender für entsprechende Überzeugungen angenommen. 


\subsection{Methode}

\subsubsection{Stichprobe und Untersuchungsdesign}

Die Stichprobe bestand aus $N=110$ Lehramtsstudierenden einer deutschen Universität, davon waren 30 männlich und 78 weiblich (bei zwei fehlten die Geschlechtsangaben). Die Studierenden dreier unterschiedlicher universitärer Veranstaltungen wurden $\mathrm{zu}$ ihren Überzeugungen zum Lehren und Lernen befragt. Entsprechend der Studienordnung der Universität bestimmten die Studierenden den Zeitpunkt für das Absolvieren dieser Veranstaltungen selbst, daher waren die Studierenden in ihrem Studium unterschiedlich weit fortgeschritten. Für die späteren Analysen wurden der Anzahl der studierten Semester entsprechend drei Gruppen gebildet: 62 Studierende befanden sich in den ersten zwei Fachsemestern ihres Hauptfaches am Anfang des Studiums, 17 zwischen dem dritten und dem fünften Fachsemester und 27 waren mit sechs bis 13 Fachsemestern am Ende des Studiums (bei 4 Personen fehlte die Angabe). Zehn Probanden studierten zwei naturwissenschaftliche Fächer wie zum Beispiel Biologie, Mathematik oder Chemie, 78 studierten zwei geisteswissenschaftliche Fächer wie beispielsweise Geschichte, Theologie oder Englisch, und 22 studierten ein naturwissenschaftliches und ein geisteswissenschaftliches Fach.

Die Datenerhebung erfolgte auf freiwilliger Basis. Die Dauer für das Ausfüllen des Fragebogens betrug ca. 40 Minuten.

\subsubsection{Instrumente}

Fragebogen zur Erfassung der vier Überzeugungen zum Lehren und Lernen (engl. The Questionnaire of Four Teachers'Beliefs - Q4TB)

Im Rahmen der Studie wurde der Q4TB eingesetzt. Der Itemstamm lautete für alle Items „Unterrichten bedeutet für mich...“. Beispielitems sind in Tabelle 14 aufgeführt. Die einzelnen Items waren auf einer fünfstufigen Skala (von 1 für ,,trifft nicht zu“ bis 5 für ,trifft zu“) zu beantworten. Dieser Fragebogen wurde bereits in einer Studie (Studie 2: Schlichter et al., in Vorbereitung) validiert und wies gute psychometrische Eigenschaften auf (Transmission: $\alpha=.85$; Konstruktion: $\alpha=.72$; Partizipation: $\alpha=.84$; Schülerorientierung $\alpha=$ .88 ), sodass er eine reliable und valide Erfassung der vier Lehrerüberzeugungen erlaubt. 
Für die vorliegende Stichprobe wurde anhand einer Faktorenanalyse überprüft, ob die vier angenommenen Überzeugungen voneinander unterschieden werden können. Die Ergebnisse bestätigten die 4-Faktoren-Struktur. Die gebildeten Skalen besitzen gute interne Konsistenzen. Eine Tabelle mit der Faktorladungsmatrix und eine Tabelle mit den Mittelwerten, Standardabweichungen und Trennschärfen der Items befinden sich im Anhang C und D.

Tabelle 14 zeigt die vier Skalen mit jeweils einem Beispielitem, die Anzahl der verwendeten Items sowie die internen Konsistenzen der vier Skalen.

\section{Tabelle 14}

Skalen der lehr-/lernbezogenen Überzeugungen, Beispielitems, Anzahl der verwendeten Items (n) sowie Reliabilitäten $(\alpha)$

\begin{tabular}{|c|c|c|c|}
\hline Skalen & Beispielitem: Unterrichten bedeutet für mich... & $n$ & $\alpha$ \\
\hline Transmission & $\begin{array}{l}\text {... Lerninhalte zu präsentieren, sodass sie von } \\
\text { den Schülerinnen und Schülern gut } \\
\text { aufgenommen werden können. }\end{array}$ & 10 & .80 \\
\hline Konstruktion & $\begin{array}{l}\text {... auch Fehler der Schülerinnen und Schüler } \\
\text { zu akzeptieren und sie weitermachen zu lassen, } \\
\text { bis sie selbst gemerkt haben, dass etwas nicht } \\
\text { stimmt. }\end{array}$ & 11 & .82 \\
\hline Partizipation & $\begin{array}{l}\text {... zum Aufbau einer Lerngemeinschaft } \\
\text { beizutragen. }\end{array}$ & 10 & .90 \\
\hline Schülerorientierung & $\begin{array}{l}\text {...sich auch Zeit für Schülerinnen und Schüler } \\
\text { zu nehmen, wenn sie etwas bereden wollen. }\end{array}$ & 10 & .84 \\
\hline
\end{tabular}

Für die vier Überzeugungen ließen die Befunde geschlechts- und fächerkombinationsspezifische Unterschiede in den Überzeugungen erkennen. So zeigten Studentinnen eine Präferenz für die Partizipation sowie tendenziell höhere Werte in der Transmission als es bei Studenten der Fall war. Studierende der Geisteswissenschaften wiesen etwas höhere Werte in der Überzeugung Konstruktion als die gemischte Gruppe auf und Studierende der Naturwissenschaften etwas höhere Werte in Transmission als ihre Kommilitonen in den geisteswissenschaftlichen Fächern. Genau wie in der ersten Validierungsstudie zeigte sich auch hier die Sensitivität des Fragebogens gegenüber Geschlechts- sowie Fächerkombinationseffekten der lehr-/lernbezogenen Überzeugungen. 
Tabelle 15 veranschaulicht die Mittelwerte und Standardabweichungen der Skalen sowie die Interkorrelationen zwischen den Skalen.

\section{Tabelle 15}

Mittelwerte $(M)$ und Standardabweichungen $(S D)$ und Interkorrelationen der Skalen

\begin{tabular}{lcccc}
\hline & $M(S D)$ & Schülerorientierung & Transmission & Konstruktion \\
\hline Partizipation & $4.30(.51)$ & $.49^{*}$ & .19 & $.49^{*}$ \\
Schülerorientierung & $3.71(.57)$ & & $.31^{*}$ & $.42^{*}$ \\
Transmission & $4.21(.50)$ & & & .04 \\
Konstruktion & $3.69(.48)$ & & & \\
\hline
\end{tabular}

$* p<.001$.

Es zeigten sich deskriptive Unterschiede zwischen den Mittelwerten der vier Skalen und folgende Korrelationsmuster: Während die Korrelationskoeffizienten zwischen Partizipation und Schülerorientierung $(r=.49, p<.001)$ sowie Partizipation und Konstruktion $(r=.49, p<.001)$, aber auch zwischen Konstruktion und Schülerorientierung $(r=.42, p<$ .001) im oberen mittleren Bereich lagen, zeigten sich Korrelationskoeffizienten zwischen Transmission und Schülerorientierung $(r=.31, p<.001)$ im mittleren Bereich und zwischen Transmission und Partizipation im unteren Bereich $(r=.19, p=.06)$, wobei hier auch die Signifikanzgrenze knapp verfehlt wurde. Interessanterweise scheinen Transmission und Konstruktion $(r=.04, p=.72)$ nicht zusammenzuhängen.

Insgesamt zeigte sich, dass die Überzeugungen Partizipation, Konstruktion und Schülerorientierung in einer engeren Beziehung zueinander standen und die Verbindung von Transmission und Schülerorientierung weniger ausgeprägt war. Die beiden Überzeugungen Transmission und Konstruktion sowie Transmission und Partizipation schienen distinkt zu sein, da sie nicht miteinander korrelieren. Somit setzte sich Transmission von den anderen drei Überzeugungen ab.

\section{Epistemologische Überzeugungen}

Neben den Items zur Erfassung der vier lehr-/lernbezogenen Überzeugungen wurde ein Fragebogen zur Messung epistemologischer Überzeugungen eingesetzt. Es wurde die von Renkl und Gruber (1995) ins Deutsche übersetzte Version (nach Salhab, 2003) des SEQ (Schommer, 1990) verwendet. Insgesamt besteht diese reduzierte Version des Instruments aus 
28 Items, wodurch eine ökonomischere Gestaltung der Erhebung und die Vermeidung von Nebeneffekten wie zum Beispiel Ermüdung möglich war.

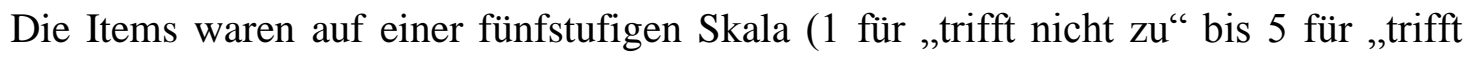
$\mathrm{zu}^{\text {“) }} \mathrm{zu}$ beantworten, wobei sie so formuliert wurden, dass sie naive Überzeugungen wiedergaben. Somit bedeutete eine hohe Zustimmung zu den Items naive und eine niedrige Zustimmung fortgeschrittene epistemologische Überzeugungen. Der Fragebogen umfasste fünf Skalen: 1) Struktur des Wissens (Beispielitem: „Die meisten Wörter haben eine klare Bedeutung“), 2) Sicherheit des Wissens (Beispielitem: „Wissenschaftler können letztlich zur Wahrheit kommen“), 3) Quelle des Wissens (Beispielitem: „Auch der Rat von Fachleuten ist oft anzuzweifeln“), 4) Lernfähigkeit (Beispielitem: „Unterschiede in der Lernfähigkeit sind angeboren“) und 5) Lerngeschwindigkeit (Beispielitem: „Erfolgreiche Studenten verstehen Lerninhalte schnell“). Die ersten drei Skalen können als allgemeinere epistemologische Überzeugungen aufgefasst werden, während die letzten beiden Skalen zur Lernfähigkeit sowie zur Lerngeschwindigkeit als lernspezifische epistemologische Überzeugungen $\mathrm{zu}$ verstehen sind. In der verwendeten Version waren 11 Items mit einer negativen Valenz (Beispielitem: „Es liegt am jeweiligen Studenten, wie viel er aus dem Lehrbuch lernt“) und 17 Items mit einer positiven Valenz (Beispielitem: „Wenn ich lerne, präge ich mir spezifische Fakten ein“) versehen, wobei hohe Werte naive Überzeugungen wiedergeben. Tabelle 16 präsentiert die aus den fünf Skalen aggregierte Gesamtskala sowie die fünf Skalen mit jeweils einem Beispielitem, die Anzahl der verwendeten Items sowie die internen Konsistenzen. 


\section{Tabelle 16}

Skalen der epistemologischen Überzeugungen, Beispielitems, Anzahl der verwendeten Items (n) sowie Reliabilitäten $(\alpha)$

Skalen

Beispielitem

Aggregation der fünf Subskalen

Epistemologische

Überzeugungen

Struktur des Wissens

Sicherheit des Wissens

Quelle des Wissens

Lernfähigkeit

Unterschiede in der Lernfähigkeit sind angeboren.

Die meisten Wörter haben eine klare Bedeutung.

Wissenschaftler können letztlich zur Wahrheit 2

kommen.

Auch der Rat von Fachleuten ist oft anzuzweifeln. ${ }^{(-)} \quad 2$ n

$\alpha$

$22 \quad .68$

Lerngeschwindigkeit

Erfolgreiche Studenten verstehen Lerninhalte schnell. 3

.25

Anmerkungen. Mit ${ }^{(-)}$markierte Items weisen eine negative Valenz für die Naivität der epistemologischen Überzeugungen auf. Das heißt, sie repräsentieren elaborierte Epistemologien und werden bei den Auswertungen umgepolt.

Die Reliabilitäten der ersten drei Skalen wiesen zufriedenstellende Werte auf (zwischen $\alpha=.62$ und $\alpha=.72$ ). Dagegen erzielten die beiden lernspezifischen Skalen Lernfähigkeit und Lerngeschwindigkeit - niedrige interne Konsistenzen ( $\alpha=.40$ und $\alpha=.25)$, sodass diese Skalen in den weiteren Analysen nicht verwendet werden.

\subsubsection{Umgang mit fehlenden Werten}

Der Anteil an fehlenden Werten ist in der vorliegenden Arbeit sehr gering (bei zwei Variablen fehlte die Angabe bei jeweils einer Person, bei der Variable Gechlecht bei 3 Personen, bei der Variable Semesteranzahl bei 4 Personen). Wie bereits in der ersten Fragebogenstudie wurden diese als missing at random verstanden. Bei der Analyse fehlender Werte konnten keine spezifischen Muster festgestellt werden, sodass von keinem systematischen Auftreten ausgegangen werden kann. Aufgrund der vergleichsweise kleinen Stichprobe $(N=110)$ wurden die fehlenden Werte mit der Methode des paarweisen 
Fallausschlusses (pairwise deletion) behandelt. Dadurch sollte die Reduktion des Datensatzes gering gehalten und eine bessere Analyse der Faktorstruktur erzielt werden (Bortz, 2005). 


\subsection{Ergebnisse}

6.6.1 Zusammenhang zwischen epistemologischen Überzeugungen und den lehr/lernbezogenen Überzeugungen Transmission, Konstruktion, Partizipation und Schülerorientierung

Die zentrale Frage dieser Studie ist die Frage nach der Beziehung der lehr/lernbezogenen Überzeugungen Transmission, Konstruktion, Partizipation und Schülerorientierung mit epistemologischen Überzeugungen. Dabei wurde erwartet, dass Transmission mit naiven epistemologischen Überzeugungen und Konstruktion mit fortgeschrittenen epistemologischen Überzeugungen zusammenhängen. Zudem wurde angenommen, dass Partizipation mit fortgeschrittenen epistemologischen Überzeugungen in Verbindung steht und Schülerorientierung dagegen unabhängig vom Konstrukt epistemologischer Überzeugungen ist. Mittels korrelativer Zusammenhänge zwischen den vier Skalen der lehr-/lernbezogenen Überzeugungen und den Skalen der epistemologischen Überzeugungen (Struktur, Sicherheit und Quelle des Wissens) wurden diese Hypothesen statistisch überprüft (Tab. 16). Zudem wurde für den Einfluss des Zeitpunkts im Studium kontrolliert, indem die Variable „Zeitpunkt im Studium“ als Kontrollvariable in die Partialkorrelation aufgenommen wurde.

\section{Tabelle 17}

Korrelationskoeffizienten der Überzeugungen zum Lehren und Lernen und den Skalen sowie der Gesamtskala zu epistemologischen Überzeugungen

\begin{tabular}{lcccc}
\hline & Partizipation & Konstruktion & Transmission & Schülerorientierung \\
\hline $\begin{array}{l}\text { Epistemologische } \\
\text { Überzeugungen }\end{array}$ & -.05 & $-.35^{* *}$ & $.29 * *$ & -.06 \\
$\begin{array}{l}\text { Struktur des } \\
\text { Wissens }\end{array}$ & -.07 & $-.33^{* *}$ & $.28^{* *}$ & -.06 \\
$\begin{array}{l}\text { Sicherheit des } \\
\text { Wissens }\end{array}$ & .01 & $-.22^{*}$ & .14 & -.06 \\
Quelle des Wissens & -.04 & $-.22^{*}$ & .11 & -.12 \\
\hline$* * p<.01 * p<.05$. & & & &
\end{tabular}


Die Interkorrelationen bestätigen die formulierten Erwartungen weitestgehend. Konstruktion hing negativ mit den Skalen epistemologischer Überzeugungen zusammen. Es zeigten sich signifikante Korrelationskoeffizienten im mittleren Bereich zwischen Konstruktion und der Gesamtskala epistemologischer Überzeugungen $(r=-.35, p<.01)$ sowie den Skalen „Struktur des Wissens“ ( $r=-.33, p<.01)$, „Sicherheit des Wissens“ ( $r=-.22, p$ $<.05)$ und „Quelle des Wissens“ ( $r=-.22, p<.05)$, wobei die letzten beiden Koeffizienten etwas niedriger ausfielen. Erwartungsgemäß wurden positive Korrelationen zwischen Transmission und der Gesamtskala epistemologischer Überzeugungen $(r=.29, p<.01)$ sowie der Skala „Struktur des Wissens“ ( $r=.28, p<.01)$ ermittelt. Die Korrelationskoeffizienten zwischen den epistemologischen Überzeugungen und den Überzeugungen Partizipation und Schülerorientierung verfehlten die Signifikanzgrenze. Dies kann als ein Hinweis auf die Distinktheit der Konstrukte aufgefasst werden.

\subsubsection{Unterschiede in den Überzeugungen zum Lehren und Lernen in Abhängigkeit vom Zeitpunkt im Studium}

Weiterhin wurde in der vorliegenden Studie der Einfluss des Zeitpunkts im Studium auf die vier lehr-/lernbezogenen Überzeugungen analysiert. Dafür wurden die Teilnehmer nach der Anzahl der studierten Semester in drei Gruppen aufgeteilt: Die Gruppe „Anfang des Studiums“ bildeten die Studierenden, die sich in den ersten zwei Semestern befanden; die Gruppe „Mitte des Studiums“ umfasste die Studierenden im dritten bis fünften Semester und die Gruppe am „Ende des Studiums“ bildeten die Studierenden mit mindestens sechs Semestern. Die Mittelwerte und Standardabweichungen der vier Skalen für diese drei Gruppen sind in Tabelle 18 dargestellt. 


\section{Tabelle 18}

Mittelwerte $(M)$ und Standardabweichungen $(S D)$ der vier Überzeugungen für die drei Gruppen: Studierende am Anfang, in der Mitte und am Ende des Studiums

\begin{tabular}{lcccccc}
\hline & \multicolumn{5}{c}{ Studierende } \\
& am Anfang $(N=62)$ & $\begin{array}{c}\text { in der Mitte }(N=17) \\
\text { des Studiums }\end{array}$ & am Ende $(N=27)$ \\
\cline { 2 - 7 } & $M$ & $S D$ & $M$ & $S D$ & $M$ & $S D$ \\
\hline Partizipation & 4.28 & .48 & 4.29 & .54 & 4.35 & .51 \\
Konstruktion & 3.66 & .45 & $3.50 *$ & .43 & $3.86^{*}$ & .51 \\
Transmission & 4.19 & .51 & 4.19 & .43 & 4.22 & .54 \\
Schülerorientierung & 3.73 & .55 & 3.58 & .70 & 3.72 & .50 \\
\hline$* p<.05$ & & & & &
\end{tabular}

Die deskriptiven Unterschiede zwischen den Studierenden der drei Gruppen wurden mittels einer multivariaten Varianzanalyse (MANOVA) auf Signifikanz überprüft. Entsprechend den Erwartungen zeigte sich ein signifikanter Unterschied in der Überzeugung Konstruktion $(F(2)=3.25, p<.05, \eta 2=.06)$. Anschließend wurden mit dem paarweisen Gruppenvergleich mittels des Tukey-Tests die Unterschiede zwischen den einzelnen Gruppen „Anfang“, „Mitte“ und „Ende des Studiums“ analysiert. Die Ergebnisse zeigten, dass, verglichen mit Studierenden in der Mitte des Studiums, Studierende am Ende des Studiums signifikant höhere Werte in der Überzeugung Konstruktion zeigten (Cohen's $d=.76$ ). Allerdings muss auch hier auf die kleine Stichprobengröße der Studierenden der drei Gruppen hingewiesen werden. Es befanden sich nur 17 Studierende in der Mitte des Studiums, somit ist auch hier die Testpower als stark eingeschränkt zu beurteilen.

Zusätzlich berechnete Regressionen zwischen der Anzahl der studierten Semester und den Werten in den vier Überzeugungen bestätigen dieses Ergebnis. Es zeigten sich signifikante Zusammenhänge zwischen der Anzahl studierter Semester und den Werten in der Überzeugung Konstruktion $(\beta=.24, p<.05)$ sowie auch zwischen der Anzahl studierter Semester und den Werten in der Überzeugung Partizipation $(\beta=.23, p<.05)$. 


\subsection{Diskussion}

Das Anliegen der vorliegenden Studie bestand darin, die Beziehung zwischen epistemologischen und lehr-lernbezogenen Überzeugungen $\mathrm{zu}$ untersuchen. Die zentrale Fragestellung lautete, ob es einen Zusammenhang zwischen den epistemologischen und den lehr-/lernbezogenen Überzeugungen Transmission, Konstruktion, Partizipation und Schülerorientierung gibt. Eine weitere Frage bezog sich auf die Unterschiede in den lehr/lernbezogenen Überzeugungen in Abhängigkeit vom Zeitpunkt im Studium. Zudem wurde der Fragebogen Q4TB eingesetzt, der in einer früheren Studie bereits validiert wurde und im Rahmen dieser Studie weitere Validierungshinweise erzielte.

\subsubsection{Fragebogen zur Messung der vier Lehrerüberzeugungen zum Lehren und Lernen}

Der zur Beantwortung der Hauptfrage eingesetzte Fragebogen Q4TB wurde zur Messung der vier lehr-/lernbezogenen Überzeugungen - Transmission, Konstruktion, Partizipation und Schülerorientierung - entwickelt und bereits in einer früheren Validierungsstudie analysiert (Studie 2). Er wies gute psychometrische Eigenschaften auf, sodass mit seiner Hilfe eine reliable und valide Erfassung der vier Lehr-Lern-Überzeugungen möglich war (Studie 2: Schlichter et al., in Vorbereitung). Im Rahmen der vorliegenden Studie wurden erneut Validierungshinweise für diesen Fragebogen gesichert. Die durchgeführten Faktorenanalysen bestätigten die 4-Faktoren-Struktur und die vier postulierten Überzeugungen. Die gebildeten Skalen wiesen gute interne Konsistenzen auf: Cronbachs Alphas der Skalen lagen im Bereich zwischen $\alpha=.80$ und $\alpha=.90$. Die Interkorrelationsmuster der vier Skalen deckten folgende Zusammenhänge zwischen den vier Überzeugungen auf: Während die meisten Korrelationen im mittleren und oberen mittleren Bereich ausfielen, schienen die Überzeugungen Transmission und Konstruktion sowie Transmission und Partizipation nicht zusammenzuhängen. Die Korrelationskoeffizienten zwischen den Überzeugungen Partizipation, Konstruktion und Schülerorientierung wiesen eine engere Beziehung dieser drei Überzeugungen untereinander auf und zeigten ebenfalls eine Verbindung zwischen Transmission und Schülerorientierung. Diese Korrelationsmuster bestätigen die Ergebnisse der Studie zur Konstruktion und Validierung des Fragebogens (Studie 2). Die Überzeugungen Partizipation, Konstruktion und Schülerorientierung befinden sich in einer engeren Beziehung zueinander, während Transmission und Konstruktion distinkt zu sein scheinen. 
Ähnlich wie in der ersten Studie kann die Vermutung geäußert werden, dass die Erklärung für dieses Korrelationsmuster in den Inhalten der Überzeugungen liegt. Während Transmission den Fokus auf Lerninhalte richtet, legen die anderen drei den Fokus auf die Schülerinnen und Schüler, auf die Lehrer-Schüler-Beziehung oder auf die Lerngemeinschaft. Möglicherweise deutet die engere Beziehung zwischen den drei Überzeugungen auf eine übergeordnete Überzeugungsdimension hin. Die Struktur der Überzeugungen ließe sich dann in zwei distinkten Lehr-Lern-Überzeugungen höherer Ordnung vorstellen - auf der einen Seite die lerninhaltsbezogene Transmission und auf der anderen Seite eine Vorstellung, bei der das Lernen nicht aus der Sicht des Lehrers und mit dem Fokus auf den Lernstoff, sondern aus der Schülersicht betrachtet wird: Was geht im Kopf des Schülers vor (konstruktivistische Vorstellungen)? Welche Lernereignisse sind in der Lerngemeinschaft wichtig (partizipatorische Vorstellungen) und wie kann die Beziehung des Lehrers zum Schüler aussehen, um der Schülerpersönlichkeit entsprechend das Lernen $\mathrm{zu}$ fördern (schülerorientierte Vorstellungen)?

Die Analysen der geschlechts- und fächerkombinationsspezifischen Unterschiede in den Überzeugungen bestätigten die Sensitivität des Fragebogens gegenüber diesen Effekten. Studentinnen zeigten höhere Werte in der Überzeugung Partizipation, was die Ergebnisse der Validierungsstudie bestätigte, und tendenziell höhere Werte in Transmission, was den Ergebnissen der Studie von Collins et al. (2001) entsprach. Des Weiteren wiesen Studierende der Geisteswissenschaften höhere Werte in der Überzeugung Konstruktion auf - verglichen mit Studierenden eines geisteswissenschaftlichen und naturwissenschaftlichen Faches. Dieses Ergebnis bestätigte das Ergebnis der Studie von Collins et al. (2001). Dagegen schienen Studierende der Naturwissenschaften die Überzeugung Transmission zu bevorzugen, was ebenfalls die Ergebnisse der Studien von Collins et al. (2001) und Norton et al. (2005) bestätigte.

An dieser Stelle soll zur Stichprobenzusammensetzung kritisch angemerkt werden, dass sich diese aus 30 männlichen und 78 weiblichen Probanden sowie 10 Studierenden der Naturwissenschaften, 78 der Geisteswissenschaften und 22 Studierenden mit der Fächerkombination aus beiden Fachgruppen zusammensetzte. Die Unterschiede in der Untergruppengröße sowie die Probandenanzahl in den Untergruppen wirkten sich einschränkend auf die Testpower aus. 
6.7.2 Zusammenhang zwischen epistemologischen Überzeugungen und den lehr/lernbezogenen Überzeugungen Transmission, Konstruktion, Partizipation und Schülerorientierung

Beide Konstrukte - lehr-/lernbezogene und epistemologische Überzeugungen werden als Facetten professioneller Lehrerkompetenzen definiert (Kunter et al., 2011) und sind somit für die Lehrerforschung und Lehrerbildung von Bedeutung. Gleichwohl wurden in der bisherigen Forschung Diskrepanzen in der Operationalisierung dieser Konstrukte festgestellt (Dubberke et al., 2008). Daraus resultierte die mangelnde Aufmerksamkeit für den Zusammenhang epistemologischer Überzeugungen und lehr-/lernbezogener Überzeugungen. Vor allem in Bezug auf die beiden bislang wenig untersuchten Überzeugungen Partizipation und Schülerorientierung trifft diese Feststellung zu. In der vorliegenden Studie wurde versucht, diesem Forschungsbedarf nachzugehen.

Die zentrale Frage der vorliegenden Studie bezog sich auf den Zusammenhang zwischen epistemologischen Überzeugungen und lehr-/lernbezogenen Überzeugungen Transmission und Konstruktion - sowie vor allem auf die wenig untersuchten Überzeugungen Partizipation und Schülerorientierung. Die Ergebnisse der vorliegenden Untersuchung bestätigten zum großen Teil die theoretischen Annahmen. So konnten für die Überzeugungen Transmission und Konstruktion Korrelationsmuster ermittelt werden, die die auf der Basis bisheriger Forschung formulierten Erwartungen bestätigten. Hohe Werte in der Überzeugung Transmission gingen mit naiven epistemologischen Überzeugungen einher, während hohe Werte in Konstruktion mit fortgeschrittenen epistemologischen Überzeugungen zusammenhingen. Diese Ergebnisse stehen im Einklang mit der Studie von Chan und Elliott (2004), die eine Verbindung zwischen traditionellen Lehr-/Lernvorstellungen und naiven epistemologischen Überzeugungen sowie zwischen konstruktivistischen Lehr/Lernvorstellungen und fortgeschrittenen epistemologischen Überzeugungen feststellten.

Entsprechend der formulierten Hypothese schien die lehr-/lernbezogene Überzeugung Schülerorientierung nicht mit den epistemologischen Überzeugungen zusammenzuhängen. Die Schülerorientierung richtet das Hauptaugenmerk auf die Schülerpersönlichkeit mit ihren Bedürfnissen und stellt somit motivationale Aspekte des Lernens in den Vordergrund, sodass kognitive Aspekte wie das Wissen als weniger wichtig erscheinen. Daher lag die Vermutung nahe, die Schülerorientierung hänge nicht mit epistemologischen Überzeugungen zusammen. Die Ergebnisse der vorliegenden Studie bestätigen diese Beziehung. 
Entgegen den Erwartungen konnte kein Zusammenhang zwischen der Überzeugung Partizipation und den epistemologischen Überzeugungen identifiziert werden. Ausgehend vom Lerngemeinschaftsansatz (Lave \& Wenger, 1991), in dem die Wissenskonstruktion bzw. die Aushandlung eines gemeinsamen Wissensverständnisses innerhalb einer Lerngemeinschaft einen zentralen Punkt darstellt, wurde im Vorfeld der Studie vermutet, dass die Überzeugung Partizipation positiv mit fortgeschrittenen epistemologischen Überzeugungen zusammenhängt, die zum Beispiel das Wissen als komplex und unsicher verstehen sowie statt einer Expertengläubigkeit eine kritische Einstellung gegenüber der Expertenmeinung aufweisen. Das erzielte Ergebnis konnte diese Vermutungen nicht bestätigen. Die Überzeugung Partizipation schien mit epistemologischen Überzeugungen in keinem Zusammenhang zu stehen. Daher kann die Annahme der „Überzeugungssyndrome“, wie sie in der COACTIV-Studie präsentiert werden, kritisch gesehen werden. Es ist also denkbar, dass die angenommenen charakteristischen Muster der epistemologischen und lehr/lernbezogenen Überzeugungen nicht für die Überzeugungen Partizipation und Schülerorientierung zutreffen. Daher sollte eine integrative Betrachtung der epistemologischen und lehr-/lernbezogenen Überzeugungen abgelegt werden und eine separate Konzeptualisierung der Begriffe sowie der entsprechenden Untersuchungsdesigns angestrebt werden. Trotz der empirisch demonstrierten Beziehung zwischen epistemologischen Überzeugungen und den Überzeugungen Transmission und Konstruktion kann bei den Überzeugungen Partizipation und Schülerorientierung die Annahme getroffen werden, dass diese beiden Überzeugung inhaltlich unabhängig von den Vorstellungen von Wissen sind und sich auf andere wissens- und lerninhaltsunabhängige Aspekte beziehen.

Es können allerdings auch Erklärungen für die nicht identifizierten Zusammenhänge zwischen epistemologischen Überzeugungen und der Überzeugung Partizipation sowie der Schülerorientierung vermutet werden, die im Untersuchungsdesign begründet liegen können. Da die Stichprobe mit $N=110$ Probanden relativ klein war, konnten die weniger starken Korrelationen zwischen epistemologischen Überzeugungen und den Überzeugungen Partizipation und Schülerorientierung möglicherweise nicht signifikant werden. Schließlich kann es möglich sein, dass in der Konzipierung der Überzeugung Partizipation, so wie sie in dem verwendeten Fragebogen getroffen wurde, die Idee der Wissenskonstruktion innerhalb einer Lerngemeinschaft nicht genügend Aufmerksamkeit bekommen hat und sich die partizipatorische Überzeugung eher über Ideen der Gemeinschaft mit deren Identifikationsmotiven und der gegenseitigen Unterstützungsmöglichkeit darstellt, so dass 
diese Darstellung der partizipatorischen Überzeugung nur wenige Berührungspunkte mit der Wissensvorstellung hat und dadurch die Zusammenhänge nicht signifikant wurden.

Zusammenfassend kann festgehalten werden, dass die Ergebnisse der vorliegenden Studie wie folgt ausfielen: Transmissive lehr-/lernbezogene Überzeugungen werden mit einem Wissensverständnis als einfach, sicher, stabil und einem Vertrauen in die Expertenmeinung in Verbindung gebracht und konstruktivistische lehr-/lernbezogene Überzeugungen mit einem Wissensverständnis als komplex, unsicher und veränderbar sowie mit einer kritischen Einstellung gegenüber der Expertenmeinung. Dagegen geben die fehlenden Zusammenhänge zwischen den lehr-/lernbezogenen Überzeugungen Partizipation und Schülerorientierung und den epistemologischen Überzeugungen einen Hinweis auf die Distinktheit dieser Konstrukte.

\subsubsection{Unterschiede in den Überzeugungen zum Lehren und Lernen in Abhängigkeit vom Zeitpunkt im Studium}

Die Ergebnisse der vorliegenden Untersuchung zeigten Unterscheide in den Überzeugungen zum Lehren und Lernen in Abhängigkeit vom Zeitpunkt im Studium. In Übereinstimmung mit den Ergebnissen der Studie 2 der vorliegenden Arbeit, in welcher Masterstudierende im Vergleich zu Bachelorstudierenden die Überzeugungen Partizipation präferierten, konnte in der vorliegenden Studie ein Anstieg der Werte in Partizipation mit der steigenden Anzahl studierter Semester nachgewiesen werden. Zudem wurden bei den Studierenden am Ende des Studiums höhere Werte in Konstruktion festgestellt. Dies kann als ein Hinweis darauf aufgefasst werden, dass sich die Studierenden im Verlauf des Studiums immer mehr Kenntnisse über Konstruktivismus sowie den Lerngemeinschaftsansatz (Lave \& Wenger, 1991) aneigneten und dementsprechend ihre Ansichten in Richtung konstruktivistischer und partizipativer Lehr-Lern-Überzeugungen änderten. 


\subsection{Grenzen der Studie und zukünftige Forschungsfragen}

Die hier präsentierte Studie hat ihre Grenzen und wirft Fragen für die weitere Forschung auf. So sind die Zusammensetzung und die Größe der vorliegenden Stichprobe nicht optimal. Vor allem in Hinblick auf die Hauptfrage der Studie bezüglich des Zusammenhangs zwischen epistemologischen und lehr-/lernbezogenen Überzeugungen wäre eine größere Stichprobe $(N$ der vorliegenden Studie $=110)$ vorteilhaft, um diese Frage mit weiteren Methoden (z.B. Strukturgleichungsmodellen) analysieren zu können.

Des Weiteren konnte die Hauptfrage aufgrund der nicht zufriedenstellenden Reliabilitäten des eingesetzten SEQ nicht ausreichend beantwortet werden. Vor diesem Hintergrund wäre der Einsatz weiterer Instrumente zur Messung epistemologischer Überzeugungen zu empfehlen. Weitere Analysen des Zusammenhangs zwischen epistemologischen und den lehr-/lernbezogenen Überzeugungen - Transmission, Konstruktion, Partizipation und Schülerorientierung - sind daher wünschenswert.

Zudem wäre eine längsschnittliche Untersuchung im Kontext der Frage nach dem kausalen Zusammenhang epistemologischer Überzeugungen und lehr-/lernbezogener Überzeugungen vorteilhaft. Damit könnten unter anderem die Ergebnisse der Studie von Chan \& Elliott (2004), die einen kausalen Effekt naiver epistemologischer Überzeugungen auf Transmission demonstrierten, verifiziert werden.

Weiterhin wäre es für die zukünftige Forschung notwendig, die mithilfe des neuen Fragebogens Q4TB messbar gemachten lehr-/lernbezogenen Überzeugungen auch bei Lehrkräften zu untersuchen. Die vorliegende Studie sowie die erste Studie zur Konstruktion und Evaluation des neu entwickelten Fragebogens Q4TB wurden ausschließlich mit Lehramtsstudierenden durchgeführt. Eine Befragung der Lehrkräfte war bislang nicht möglich und wird als dringend empfehlenswert erachtet.

Neben der weiteren Validierung könnte eine Erhebung mit Lehrkräften im Beruf Hinweise für Lehrkräfte selbst und für die Ausbilder im Lehrerberuf liefern. Diese könnten auch im Rahmen professioneller Weiterbildung genutzt werden. Als wichtig wird erachtet, dass sich angehende und erfahrene Lehrkräfte ihrer lehr-/lernbezogenen und auch epistemologischen Überzeugungen bewusst werden. Besonders im Rahmen der Diskussion um Lehrerkompetenzen werden Lehrerüberzeugungen als eine Chance aufgefasst, das Lehrerhandeln besser verstehen und erklären zu können. Daher wird die weitere Forschung von Lehrerüberzeugungen verstärkt begrüßt. 


\section{$7 \quad$ Zusammenfassende Schlussdiskussion und Ausblick}

Im folgenden Kapitel werden zunächst die zentralen Ergebnisse vor dem Hintergrund der Forschungsfragen der drei Studien (Kapitel 4-6) zusammengefasst (Abschnitt 7.1). Dabei beziehen sich die Fragen 1 und 2 auf die erste Studie (Kapitel 4), die Fragen 3 bis 6 auf die zweite Studie (Kapitel 5) und die Fragen 7 und 8 auf die dritte Studie (Kapitel 6). Daran anschließend werden die Ergebnisse integrativ diskutiert (Abschnitt 7.2), die Grenzen der Arbeit aufgezeigt und davon ausgehend mögliche Fragen für die zukünftige Forschung abgeleitet (Abschnitt 7.3).

\subsection{Zusammenfassung der zentralen Ergebnisse}

Ziel der vorliegenden Arbeit war es, Lehrerüberzeugungen zum Lehren und Lernen detailliert zu untersuchen. Im Rahmen von drei Studien sollten vier Überzeugungen zum einen mithilfe von qualitativen Methoden analysiert werden und zum anderen mithilfe eines neu entwickelten Fragenbogens zur Messung der vier Lehrerüberzeugungen zum Lehren und Lernen (Q4TB) messbar gemacht werden. Die Arbeit beinhaltete folgende Fragestellungen:

1. Inwieweit lassen sich die in der einschlägigen Literatur identifizierten Überzeugungen Transmission, Konstruktion, Partizipation und Schülerorientierung in der Selbstauskunft der (angehenden) Lehrkräfte zu ihren Ansichten zum Lehren und Lernen identifizieren und

2. inwieweit unterscheiden sich Lehrkräfte $\mathrm{zu}$ verschiedenen Zeitpunkten der Lehrerausbildung und Lehrerlaufbahn in ihren Überzeugungen zum Lehren und Lernen?

3. Lassen sich die vier Überzeugungen zum Lehren und Lernen und/oder ihre Facetten mithilfe eines quantitativen Messinstruments erfassen? Lässt sich die 4-FaktorenStruktur mittels einer exploratorischen Faktorenanalyse bestätigen? Sind die gebildeten Skalen reliabel?

4. In welchem Zusammenhang stehen die vier Überzeugungen zu Lehr-Lern-Begriffen, $\mathrm{zu}$ Erziehungszielen und zu allgemeinen epistemologischen Überzeugungen?

5. Unterscheiden sich die Lehr-Lern-Überzeugungen in Abhängigkeit vom Geschlecht und/oder vom studierten Fach der Lehramtsstudierenden? 
6. Unterscheiden sich die Lehr-Lern-Überzeugungen von Bachelor- und Masterstudierenden sowie von Studierenden am Anfang, in der Mitte und am Ende des Studiums?

7. Gibt es einen Zusammenhang zwischen epistemologischen Überzeugungen und den vier lehr-/lernbezogenen Überzeugungen Transmission, Konstruktion, Partizipation und Schülerorientierung?

Die zentralen Ergebnisse zu den Fragen 1 und 2 beziehen sich auf die Befunde der in Kapitel 4 dargestellten Interviewstudie.

(1) Inwieweit lassen sich die in der einschlägigen Literatur identifizierten Überzeugungen Transmission, Konstruktion, Partizipation und Schülerorientierung in der Selbstauskunft der (angehender) Lehrkräfte zu ihren Ansichten zum Lehren und Lernen identifizieren?

Entsprechend den formulierten Erwartungen wurden in den mittels Inhaltsanalyse ausgewerteten Interviews die Überzeugungen Transmission und Konstruktion vorgefunden. In den Ausführungen der Interviewten ließen sich zudem Partizipation und Schülerorientierung identifizieren. Allerdings wurden die vier Lehr-Lern-Überzeugungen unterschiedlich detailliert thematisiert. Dies wurde mithilfe der quantifizierenden Analyse der Interviews demonstriert, bei welcher die Anzahl von Einzelaussagen (Analyseeinheiten) in einem Interview und die Anzahl einzelner Aspekte einer Überzeugung (gebildete Unterkategorien) bestimmt wurden. Die Überzeugung Transmission dominierte in den Interviews sowohl in Hinblick auf Einzelaussagen als auch auf einzelne Aspekte. In den Einzelinterviews sprach die Mehrheit der Interviewten grundsätzlich von allen vier Überzeugungen, aber dennoch wurden im Mittel pro Interview häufiger Aussagen über Transmission als über die anderen Überzeugungen geäußert. Dieses erste Ergebnis zeigte, dass - verglichen mit den übrigen drei - die traditionellere Überzeugung Transmission in den Vorstellungen der interviewten Lehrkräfte zum Lehren und Lernen überwog.

Weiterhin wurde für jede Person die dominierende Überzeugung bestimmt, um festzustellen, inwieweit eine der vier Überzeugungen mehr Zuspruch unter den Interviewten fand. Insgesamt zeigten diese Analysen, dass die Mehrheit der Interviewten (32 von 50) über eine dominierende Überzeugung verfügte. 12 Interviewte bevorzugten die Überzeugung Transmission, dagegen nur acht die Überzeugung Partizipation, sechs die Überzeugung 
Konstruktion und ebenfalls sechs die Überzeugung Schülerorientierung. Somit demonstrierten auch die Analysen der dominierenden Überzeugung erneut das Überwiegen der Überzeugung Transmission in den Lehrervorstellungen.

(2) Inwieweit unterscheiden sich Lehrkräfte $\mathrm{zu}$ unterschiedlichen Zeitpunkten der Lehrerausbildung und Lehrerlaufbahn in ihren Überzeugungen zum Lehren und Lernen?

Inwieweit verschiedene Zeitpunkte in der Lehrerausbildung und Lehrerlaufbahn für die Lehr-Lern-Überzeugungen von Bedeutung sind, konnte aus unterschiedlichen Perspektiven beleuchtet werden: 2.1) bei der Analyse dominierender Überzeugungen, 2.2) im Hinblick auf den Umfang der Interviews sowie 2.3) bei der prozentuellen Analyse der Aussagen eines Interviews.

(2.1) In Abhängigkeit vom Zeitpunkt in der Lehrerausbildung und Lehrerlaufbahn schienen die Interviewten unterschiedliche Überzeugungen zu bevorzugen. In der Mitte des Studiums überwogen Konstruktion und Partizipation (2 von 10 Studierenden hatten diese Überzeugungen als ihre dominierenden); am Ende des Studiums dominierte bei den Studierenden Konstruktion (3 von 10). Referendare zeigten eine Präferenz für Transmission und Partizipation (je 2 von 10). Auffallend war die Ähnlichkeit in den Mustern dominierender Überzeugungen von Studierenden am Anfang des Studiums und von erfahrenen Lehrkräften: Beide bevorzugten Transmission (4 von 10 bzw. 5 von $10)$.

(2.2) Unterschiede im Umfang der Interviews machten deutlich, dass erfahrene Lehrkräfte, aber auch Referendare mehr über ihre Einsichten zum Lehren und Lernen berichten konnten als ihre zukünftigen Kolleginnen und Kollegen. Interviews mit Studierenden aller drei Zeitpunkte im Studium enthielten signifikant weniger Aussagen als die der Referendare und erfahrenen Lehrkräfte.

(2.3) Es zeigten sich auch Unterschiede bei der prozentualen Analyse der Aussagen zwischen den Lehrkräften zu unterschiedlichen Zeitpunkten ihrer 
Lehrerausbildung und Lehrerlaufbahn. Der Prozentsatz der einer bestimmten Überzeugung zugeordneten Aussagen wurde mit dem Prozentsatz der anderen Überzeugungen verglichen, um die Muster der Überzeugungsverläufe der fünf Gruppen miteinander zu vergleichen. Die Ergebnisse zeigten, dass Studierende am Anfang des Studiums transmissive und schülerorientierte Überzeugungen hatten, welche während des Studiums an Bedeutung verloren und mit dem Einstieg in den Beruf wieder präsenter wurden. Konstruktion und Partizipation zeigten ein entgegengesetztes Muster: Am Anfang des Studiums waren diese Überzeugungen weniger präsent, stiegen aber im Verlauf des Studiums stark an und fielen mit dem Einstieg in den Lehrerberuf wieder ab. Eine multivariate Varianzanalyse mit anschließenden Kontrasttests zeigte einen signifikanten Unterschied zwischen den fünf Gruppen in den Überzeugungen Konstruktion und Schülerorientierung. Diese zwei Überzeugungen veränderten sich demnach bedeutend in Abhängigkeit vom Zeitpunkt des Studiums und vom Zeitpunkt in der Lehrerlaufbahn. Anders als die Schülerorientierung, die im Verlauf des Studiums ihre Präferenz verlor, gewann die Konstruktion im Verlauf des Studiums an Beliebtheit. Allerdings verlor sie diese wieder mit dem Einstieg in den Lehrerberuf, während die Schülerorientierung erneut im Beruf an Bedeutung gewann.

Die oben genannten Ergebnisse bestätigen die Annahme, dass sich Lehramtsstudierende zu verschiedenen Zeitpunkten in der Lehrerausbildung und Lehrkräfte zu verschiedenen Zeitpunkten in der Lehrerlaufbahn in ihren Überzeugungen zum Lehren und Lernen unterscheiden. In Abhängigkeit von diesen Zeitpunkten weisen sie unterschiedliche Werte in allen vier Überzeugungen auf. Zudem können erfahrene Lehrkräfte mehr Aussagen über ihre Einsichten zum Lehren und Lernen machen als ihre zukünftigen Kolleginnen und Kollegen. Sie tendieren aber ebenso wie die Studierenden am Anfang des Studiums dazu, Transmission zu bevorzugen.

Die Fragen 3 bis 6 beziehen sich auf die Befunde der in Kapitel 5 dargestellten Studie zur Konstruktion und Validierung des Fragebogens zur Messung der vier Überzeugungen zum Lehren und Lernen - Transmission, Konstruktion, Partizipation und Schülerorientierung.

(3) Lassen sich die vier Überzeugungen zum Lehren und Lernen und/oder deren Facetten mithilfe eines quantitativen Messinstruments erfassen? Kann die 4-Faktoren-Struktur 
mittels einer exploratorischen Faktorenanalyse bestätigt werden? Sind die gebildeten Skalen reliabel?

Im Rahmen der zweiten Studie wurde ein Fragebogen entwickelt, um die vier LehrLern-Überzeugungen Transmission, Konstruktion, Partizipation und Schülerorientierung valide und reliabel zu messen. Die Ergebnisse der exploratorischen Faktorenanalysen bestätigten die theoretisch postulierte 4-Faktoren-Struktur. Die Reliabilitätsanalyse zeigte gute Werte sowohl für die vier als auch für die vierzehn gebildeten Skalen, die verschiedene Facetten der vier Überzeugungen darstellen. Um Aussagen über die vier Lehr-LernÜberzeugungen zu treffen, wurden weitere Analysen mit den vier Skalen durchgeführt. Obwohl die Anzahl der Probanden relativ gering war $(N=276)$, erreichten die Reliabilitäten gute Werte ( $\alpha$ zwischen .72 und .88). Somit konnten die Lehr-Lern-Überzeugungen bei Lehramtsstudierenden reliabel gemessen werden. Die Interkorrelationen zwischen den vier Skalen demonstrierten, dass sich die Überzeugungen Partizipation, Konstruktion und Schülerorientierung in einer engen Beziehung zueinander befanden, aber die Verbindung zwischen Transmission zu Partizipation sowie Schülerorientierung weniger ausgeprägt war. Zudem schienen die beiden Überzeugungen Transmission und Konstruktion distinkt zu sein, da sie nicht miteinander korrelierten. Somit setzte sich die Transmission von den anderen drei Überzeugungen ab.

(4) In welchem Zusammenhang stehen die vier Überzeugungen mit Lehr-Lern-Begriffen, mit Erziehungszielen und mit allgemeinen epistemologischen Überzeugungen?

Die Ergebnisse der Zusammenhangsanalysen deuteten insgesamt auf eine gute Konstruktvalidität des Instruments hin. Die theoretische Annahme, dass es einen positiven Zusammenhang zwischen der Überzeugung Transmission und einem instruktionsorientierten Lehrbegriff sowie zwischen der Konstruktion und der Vorstellung von Lernen als Verstehen gibt, wurde bestätigt. Die Überzeugungen Partizipation und Schülerorientierung wiesen ähnliche Korrelationen zu Lehr-Lern-Begriffe auf wie die Überzeugung Konstruktion. Zusammenhangsanalysen mit Erziehungszielen ließen auf eine enge Beziehung der Überzeugung Transmission mit Leistungs- und Anstrengungsbereitschaft, Ordnung und Disziplin sowie Kenntnissen in den Hauptfächern erkennen. Diese Erziehungsziele griffen konzeptuelle Inhalte von Transmission auf. Partizipation schien mit Erziehungszielen wie beispielsweise sozialer Verantwortungsbereitschaft, angemessenen Umgangsformen und 
Kenntnissen, die auf den Beruf vorbereiten, zusammenzuhängen. Diese Verbindungen ließen ebenfalls konzeptuelle Inhalte von Partizipation erkennen, da der hinter dieser Überzeugung stehende Lerngemeinschaftsansatz die Vorbereitung auf den Beruf als zentrales Lernziel beschreibt (Lave \& Wenger, 1991). Die Ergebnisse der Zusammenhangsanalysen wiesen somit auf die konvergente Konstruktvalidität des Fragebogens hin.

Auch Analysen des Zusammenhangs zwischen den vier Lehr-Lern-Überzeugungen und allgemeinen epistemologischen Überzeugungen, gemessen mit einer kurzen Skala, bestätigten die theoretischen Annahmen: Transmission wies eine signifikant positive Korrelation mit Dualismus auf, einer der epistemologischen Überzeugungen, die als weniger elaboriert zu verstehen ist. Dagegen hing die Überzeugung Konstruktion mit Relativismus zusammen, was einer elaborierteren epistemologischen Überzeugung entspricht.

(5) Unterscheiden sich die Überzeugungen angehender Lehrkräfte in Abhängigkeit vom Geschlecht und/oder vom studierten Fach?

Vergleiche der Lehr-Lern-Überzeugungen von Studentinnen mit den Überzeugungen ihrer männlichen Kommilitonen konnten die Sensitivität des Fragebogens gegenüber geschlechtsspezifischen Unterschieden demonstrieren: Studentinnen wiesen signifikant höhere Werte in Partizipation auf, was auf ihre Präferenz für diese Überzeugung schließen lässt. Zusätzlich zum geschlechtsspezifischen Effekt wurde auch ein fächerkombinationsspezifischer Effekt bei der Überzeugung Konstruktion nachgewiesen. Verglichen mit der Gruppe der Geisteswissenschaften und mit den Studierenden, die jeweils ein naturwissenschaftliches und ein geisteswissenschaftliches Fach studierten, wiesen Studierende der Naturwissenschaften die höchsten Werte in der Überzeugung Konstruktion auf. Mit diesen Analysen konnte eine ausreichende Sensitivität des neu entwickelten Fragebogens gegenüber geschlechts- und fächerkombinationsspezifischen Unterschieden demonstriert werden. Demnach ist es möglich, mit diesem Fragebogen zwischen weiblichen und männlichen sowie zwischen Probanden verschiedener Disziplinen zu unterscheiden.

(6) Unterscheiden sich die Lehr-Lern-Überzeugungen zwischen Bachelor- und Masterstudierenden sowie zwischen Studierenden am Anfang, in der Mitte und am Ende des Studiums? 
In Studie 2 wurden die Lehr-Lern-Überzeugungen von Bachelorstudierenden mit denen von Masterstudierenden verglichen. Das Ergebnis zeigte höhere Werte in der Überzeugung Transmission bei Bachelorstudierenden und höhere Werte in Partizipation bei Masterstudierenden. Dies mag als Hinweis auf eine Entwicklung der Lehr-LernÜberzeugungen in Verlauf des Studiums zu werten sein. Die bei den Bachelorstudierenden relativ verbreitete Überzeugung Transmission verlor ihre Beliebtheit unter Masterstudierenden, dagegen wurden die Werte hinsichtlich Partizipation bei Masterstudierenden höher.

Auch in Studie 3 wurden Lehr-Lern-Überzeugungen von Studierenden zu unterschiedlichen Zeitpunkten in der Lehrerausbildung miteinander verglichen. Hier zeigten Studierende am Ende des Studiums signifikant höhere Werte in der Überzeugung Konstruktion. Zusätzlich berechnete Regressionen zwischen der Semesteranzahl und den Werten in den vier Überzeugungen zeigten signifikante Zusammenhänge der Semesteranzahl mit den Überzeugungen Konstruktion und Partizipation. Somit ergab sich erneut ein Hinweis auf die Veränderung der Lehr-Lern-Überzeugungen, und zwar stieg die Beliebtheit von Konstruktion und Partizipation im Verlauf des Studiums an.

(7) Gibt es einen Zusammenhang zwischen epistemologischen Überzeugungen und den vier lehr-/lernbezogenen Überzeugungen Transmission, Konstruktion, Partizipation und Schülerorientierung?

Im Zentrum der dritten Studie stand die Frage nach dem Zusammenhang zwischen den vier Lehr-Lern-Überzeugungen und den epistemologischen Überzeugungen. Hierfür wurden mithilfe der Skalen des EBQ nach Schommer (1990, 1992) epistemologische Überzeugungen und mithilfe des Q4TB (Schlichter et al., in Vorbereitung) die vier Lehr-Lern-Überzeugungen Transmission, Konstruktion, Partizipation und Schülerorientierung gemessen. Die Ergebnisse der Korrelationsanalysen bestätigten die Ergebnisse der bisherigen Studien weitestgehend. Die Überzeugung Konstruktion hing negativ mit den Skalen „Struktur des Wissens“, „Sicherheit des Wissens“ und „Quelle des Wissens“, sowie der Gesamtskala epistemologischer Überzeugungen, welche naive Epistemologien wiedergeben, zusammen. Dagegen zeigten sich signifikante positive Korrelationen zwischen Transmission und der Skala „Struktur des Wissens“ sowie der Gesamtskala. Die Korrelationskoeffizienten zwischen den epistemologischen Überzeugungen und den Überzeugungen Partizipation und Schülerorientierung verfehlten die Signifikanzgrenze. 
Zusammenfassend lässt sich festhalten, dass die Korrelationsmuster die formulierten Erwartungen bestätigten: Eine hohe Zustimmung zur Überzeugung Konstruktion ging mit elaborierteren epistemologischen Überzeugungen einher und eine hohe Zustimmung zur Transmission mit naiven epistemologischen Überzeugungen. Entsprechend den Vermutungen hing Schülerorientierung nicht mit epistemologischen Überzeugungen zusammen. Wider Erwarten hingen Partizipation und epistemologische Überzeugungen nicht zusammen, was als Hinweis auf die Distinktheit der Konstrukte aufgefasst werden kann. 


\subsection{Integrative Diskussion}

Ziel der vorliegenden Arbeit war es herauszufinden, inwieweit sich die vier Überzeugungen zum Lehren und Lernen in den Einsichten angehender und erfahrener Lehrkräfte zum Lehren und Lernen identifizieren lassen und inwieweit sich die vier Überzeugungen mittels eines quantitativen Messinstruments erfassen lassen. Dabei bedurften besonders die Überzeugungen Partizipation und Schülerorientierung größerer Aufmerksamkeit, da sie in der bisherigen Lehr-Lern-Forschung kaum bzw. vorwiegend qualitativ untersucht wurden. Die empirische Forschung demonstrierte bereits die Bedeutung transmissiver und konstruktivistischer Lehrerüberzeugungen für das Lehrerhandeln und auch für die Schülerleistung (Brunner et al., 2006; Dubberke et al., 2008; Peterson et al., 1989; Staub \& Stern, 2002) ebenso wurden entsprechende Instrumente zur Messung dieser zwei Überzeugungen entwickelt (Dubberke et al., 2008; OECD, 2009). Dagegen wurden andere Überzeugungen, wie Partizipation und Schülerorientierung, weitestgehend vernachlässigt, sodass bisher unklar blieb, inwieweit sich diese beispielweise auf Lehrerhandeln und Schülerleistung auswirken. Gleichwohl existieren theoretische (Klieme et al., 2006; Sfard, 1998; Lave \& Wenger, 1991) und empirische Hinweise, die die Bedeutung dieser Lehr-LernÜberzeugungen unterstreichen (Alger, 2009; Brookhart \& Freeman, 1992; Rathbone \& Pierce, 1990; Weinstein, 1989; Wideen et al., 1998). Deshalb wurde für die vorliegende Arbeit die Ausweitung des Konstrukts von Überzeugungen auf diese zwei Lehr-LernÜberzeugungen für notwendig erachtet: Es sollten über das bereits untersuchte Überzeugungspaar Transmission-Konstruktion hinaus auch partizipatorische und schülerorientierte Lehr-Lern-Überzeugungen analysiert werden. Dafür wurden in der ersten empirischen Studie Interviews durchgeführt (1).

Auf der Grundlage dieser Interviewstudie sowie auf der Basis theoretischer Überlegungen und in Anlehnung an bereits existierende Instrumente (bei Transmission, Konstruktion und teilweise Schülerorientierung) wurde ein Fragebogen entwickelt, der im Rahmen der zweiten und dritten Studie eingesetzt wurde. Die zentrale Frage der zweiten Studie bezog sich somit auf die Messbarkeit der vier Überzeugungen mithilfe des Q4TB: auf die faktorielle Struktur der entwickelten Items sowie auf die Validität und Sensitivität des Fragebogens gegenüber geschlechts- und fächerkombinationsspezifischen Unterschieden (2).

Außerdem wurde in der ersten Interviewstudie analysiert, in welcher Konstellation sich die vier Lehr-Lern-Überzeugungen während der Lehrerlaufbahn identifizieren lassen. Hierfür wurden - anders als in vielen bisherigen Untersuchungen - Lehramtsstudierende zu 
drei unterschiedlichen Zeitpunkten ihres Studiums sowie Referendare und Lehrkräfte mit mindestens fünf Jahren Lehrerfahrung interviewt. Dies ermöglichte eine differenzierte Betrachtung verschiedener Zeitpunkte der Lehrerlaufbahn mit deren Bedeutung für die LehrLern-Überzeugungen. Mit diesem querschnittlichen Design war es zwar nicht möglich, Veränderungen der Lehr-Lern-Überzeugungen zu untersuchen, aber es konnten Hinweise darauf gewonnen werden. Der Frage nach der Bedeutung der vier Überzeugungen zu verschiedenen Zeitpunkten in der Lehrerausbildung wurde ebenfalls in der zweiten und dritten Studie nachgegangen, und zwar insofern, als dass Lehr-Lern-Überzeugungen von Bachelor- und Masterstudierenden sowie von Studierenden am Anfang, in der Mitte und am Ende ihres Studiums miteinander verglichen wurden (3).

Im Rahmen der dritten Studie wurde die Frage nach dem Zusammenhang zwischen epistemologischen und lehr-/lernbezogenen Überzeugungen geklärt. Die Forschung lieferte bereits Hinweise auf Zusammenhänge epistemologischer Überzeugungen mit lehr/lernbezogenen Überzeugungen, allerdings lediglich für Transmission und Konstruktion. Da die übrigen Lehr-Lern-Überzeugungen bislang nicht quantitativ untersucht wurden, war die Beantwortung der Frage nach dem Zusammenhang zwischen naiven bzw. fortgeschrittenen epistemologischen Überzeugungen und partizipatorischen sowie schülerorientierten Überzeugungen bislang nicht möglich. Mithilfe des Q4TB konnten diese Zusammenhänge analysiert werden (4). Im Folgenden sollen die vier für die vorliegende Arbeit zentralen Aspekte in Bezug auf deren Gesamtertrag diskutiert werden. 
(1) Qualitative Analysen der vier Überzeugungen zum Lehren und Lernen

Ein zentraler Punkt der vorliegenden Arbeit bestand in der detaillierten qualitativen Analyse der Lehr-Lern-Überzeugungen Transmission und Konstruktion und vor allem der wenig untersuchten Überzeugungen Partizipation und Schülerorientierung. Zunächst wurde eine Übersicht über die in der Forschung bekannten Lehr-Lern-Überzeugungen erstellt. Die bei der Literaturrecherche identifizierten Überzeugungen und Konzeptionen wurden einander gegenübergestellt und ihre Inhalte miteinander verglichen. Damit konnten diese vielen Vorstellungen, die oft fast identische Inhalte beschrieben, $\mathrm{zu}$ einigen wenigen zusammengefasst werden. Dies wiederum ermöglichte eine Reduktion der Anzahl verschiedener Überzeugungen ohne einer inhaltlichen Reduzierung (siehe Anhang A).

Die Ergebnisse dieser qualitativen Studie belegen insgesamt, dass nicht nur Transmission und Konstruktion in den Vorstellungen der Lehrkräfte zum Lehren und Lernen, sondern auch mehrere Facetten der Überzeugungen Partizipation und Schülerorientierung vorzufinden sind.

(2) Messung der vier Überzeugungen zum Lehren und Lernen mithilfe eines Fragebogens

Ein weiterer zentraler Punkt der vorliegenden Arbeit bestand darin, die vier Überzeugungen zum Lehren und Lernen messbar zu machen. Im Rahmen der zweiten Studie wurde das neu entwickelte Instrument zur Erfassung von Transmission, Konstruktion, Partizipation und Schülerorientierung validiert. Die durchgeführten Faktoren- und Reliabilitätsanalysen zeigten, dass es mithilfe dieses Instruments möglich ist, die vier Überzeugungen faktoriell valide und reliabel $\mathrm{zu}$ messen. Zudem ist es mithilfe des Fragebogens möglich, geschlechts- und fächerkombinationsspezifische Unterschiede in den Lehrerüberzeugungen aufzudecken, was ebenfalls für Validität des Instruments spricht.

(3) Bedeutung der Lehr-Lern-Überzeugungen zu verschiedenen Zeitpunkten in der Lehrerlaufbahn

Viele bisherige Studien, welche die Unterschiede in den Lehr-Lern-Überzeugungen in Abhängigkeit vom Zeitpunkt der Lehrerlaufbahn untersucht haben, beschränkten sich auf ein oder zwei Messzeitpunkte. Längsschnittstudien gab es ausschließlich für die Zeit der universitären Ausbildung. In der vorliegenden Interviewstudie (Studie 1) wurden fünf 
Zeitpunkte der Lehrerlaufbahn beleuchtet: Es wurden Studierende am Anfang, in der Mitte und am Ende des Lehramtsstudiums sowie Referendare und Lehrkräfte mit mindestens fünf Jahren Berufserfahrung untersucht. Die Ergebnisse gaben Hinweise darauf, dass sich LehrLern-Überzeugungen sowohl während der universitären Ausbildung als auch danach im Lehrerberuf verändern. Die Unterschiede in den Verlaufsmustern der Überzeugungen zu den fünf Zeitpunkten demonstrierten einen Anstieg der Werte in der Überzeugung Konstruktion während der Lehrerausbildung sowie einen Rückgang dieser mit dem Einstieg in den Lehrerberuf und einen Rückgang der Werte der Überzeugung Schülerorientierung während des Studiums sowie einen Anstieg dieser nach dem Studium. Im Einklang mit diesen Ergebnissen wurden Unterschiede in den Lehr-Lern-Überzeugungen zwischen Bachelor- und Masterstudierenden im Rahmen der ersten Fragebogen-Studie (Studie 2) sowie zwischen Studierenden am Anfang und am Ende des Studiums im Rahmen der zweiten FragebogenStudie (Studie 3) identifiziert. Es zeigten sich hohe Werte in der Überzeugung Transmission bei Studierenden am Anfang des Studiums sowohl in der Interviewstudie (Studie 1) als auch in der ersten Fragebogenstudie (Studie 2). In beiden Studien verringerten sich die Werte für Transmission im Verlauf des Studiums bzw. zum Ende des Studiums hin. Dieses Ergebnis bestätigte Ergebnisse bisheriger Studien, die ebenfalls einen Rückgang von transmissiven Überzeugungen im Studium zeigten (Brookhart \& Freeman, 1992; Calderhead, 1996; Richardson, 1996; Wan $\mathrm{Ng}$ et al., 2010). Im Einklang mit den Ergebnissen früherer Untersuchungen (Hollingsworth, 1989; Leavy et al., 2007) waren die Werte der Überzeugung Konstruktion in allen drei Untersuchungen der vorliegenden Arbeit unter den Studierenden am Anfang des Studiums relativ niedrig. Sie stiegen im Verlauf des Studiums an, was sowohl die Interviewstudie als auch die zweite Fragebogenstudie (Studie 3) demonstrierten. Diese Veränderungsmuster wurden in allen drei Studien erzielt, sodass sie deren Ergebnisse gegenseitig bestätigen. Zwei weitere Veränderungen in den Lehr-Lern-Überzeugungen konnten jeweils in einer der drei Studien beobachtet werden: In der Interviewstudie (Studie 1) wurde festgestellt, dass die Überzeugung Schülerorientierung immer weniger Zustimmung im Verlauf des Studiums fand, was den Ergebnissen der Studien von Nettle (1998) und Rust (1994) entsprach, aber nicht jedoch durch die Fragebogenstudien (Studien 2 und 3) bestätigt werden konnte. Zudem wurde in der ersten Fragebogenstudie (Studie 2) festgestellt, dass nicht nur Konstruktion, sondern auch Partizipation im Verlauf des Studiums an Beliebtheit gewannen. Dies wiederum bestätigte das Ergebnis der Studie von Martínez et al. (2001). Allerdings stammten die zitierten Studien aus dem angloamerikanischen Raum sowie aus Spanien und Irland. Es könnte durchaus denkbar sein, dass die Lehrerausbildung sowie Lehr- 
Lern-Situationen und schulische Umwelten der verschiedenen Länder mit ihren kulturellen sozioökonomischen und historisch-politischen - Spezifitäten der Bildungssysteme einen Einfluss auf die Lehr-Lern-Überzeugungen ausüben können. Es ließen sich jedoch trotz dieser kulturellen Hintergründe ähnliche Veränderungsmuster in den Lehr-Lern-Überzeugungen im Verlauf des Studiums und auch der Lehrerlaufbahn beobachten.

Betrachtet man die Überzeugungsmuster der Studierenden auf der einen Seite sowie die der Lehrkräfte auf der anderen Seite, scheint auf längere Sicht Konstruktion immer weniger und Transmission immer mehr an Popularität zu gewinnen. Dieses Ergebnis der Interviewstudie steht im Einklang mit dem Ergebnis der Studie von Martínez et al. (2001), in der angehende Lehrkräfte im Vergleich zu erfahrenen Lehrkräften mehr konstruktivistische und partizipatorische Ideen äußerten. Allerdings fällt auf, dass trotz dieser positiven Veränderungen der Lehr-Lern-Überzeugungen im Verlauf des Studiums eine überraschende Ähnlichkeit in den Überzeugungsmustern erfahrener Lehrkräfte mit denen von Studierenden am Anfang des Studiums bestand. Diese Entwicklung kann als das Ausbleiben eines Langzeiteffekts der Lehrerausbildung kritisch angesehen werden. Obwohl neue Lehr-LernKonzepte wie Konstruktivismus oder der Lerngemeinschaftsansatz im Verlauf des Studiums Zugang zu den Überzeugungen zukünftiger Lehrkräfte fanden, bekam mit der beruflichen Praxis Transmission wieder die Oberhand. Martínez et al. (2001) kritisierten in diesem Kontext transmissive Überzeugungen als traditionell, was mit den Worten von Dann et al. (1981) einer immer konservativer werdenden Einstellung von angehenden Lehrkräften entspricht. Korthagen (2010) kritisiert aus diesem Grund die Lehrerausbildung in den USA, die zwar erfolgreich im Lehren von innovativen und vielversprechenden Lehr-LernKonzepten sei, die aber dennoch die Schulen nicht erreiche, sodass noch immer das traditionelle behavioristische Lernmodel den Schulalltag dominiere (siehe auch Resnick \& Hall, 1998). Der Kontrast zwischen der universitären Ausbildung und schulischer Praxis, zwischen theoretischem Wissen und praktischen Kenntnissen wird als Ursache für dieses Ausbleiben des längerfristigen Effekts gesehen (Broekkamp \& van Hout-Wolters, 2007). In den Augen einer angehenden Lehrkraft kann das akademische Wissen innerhalb des neuen schulischen Kontextes sogar in Konflikt mit der spezifischen Schulkultur und den schulischen Prioritäten geraten (Cooney, 1985; Raymond, 1997; Ruthven, 2002). So wird der Übergang von der Universität zum schulischen Berufsfeld als kritisch, mit Unsicherheiten und Diskrepanzen behaftet, beschrieben und als „Realitätsschock“ (Dann et al., 1978) bezeichnet. Dieser Realitätsschock zwingt schließlich angehende Lehrkräfte dazu, ihre innovativen Überzeugungen zugunsten von traditionellen Lehr-Lern-Vorstellungen aufzugeben und ihre 
progressiven Ideen kontinuierlich gegen traditionelle auszutauschen (Veenman, 1984; Skott, 2002).

(4) Zusammenhang zwischen epistemologischen und den lehr-/lernbezogenen

Überzeugungen Transmission, Konstruktion, Partizipation und Schülerorientierung

Der vierte zentrale Aspekt der vorliegenden Arbeit bezog sich auf den Zusammenhang zwischen epistemologischen und lehr-/lernbezogenen Überzeugungen. Die Ergebnisse der zweiten Fragebogenstudie (Studie 3) zeigten im Einklang mit der bisherigen Forschung, dass offenbar eine Verbindung zwischen naiven epistemologischen Überzeugungen und der LehrLern-Überzeugung Transmission besteht. So wurden bereits in der Validierungsstudie des Fragebogens (Studie 2) Hinweise darauf vorgefunden, dass eine transmissive Lehr-LernÜberzeugung mit einer Vorstellung von Wissen als falsch oder richtig (dualistische epistemologische Überzeugungen) und einem instruktionsorientierten Lehrbegriff zusammenhängt. In diesem Zusammenhang sprachen Olafson und Schraw (2006) sowie Seidel et al. (2008) davon, dass Lehrkräfte mit transmissiven Überzeugungen ihre Schülerinnen und Schüler als passive Rezipienten von fest definierten Wissenseinheiten sehen und daher sie selbst eine aktive Rolle im Unterricht übernehmen. Im Einklang damit zeigten sich in der hier präsentierten ersten Fragebogenstudie (Studie 2) Hinweise darauf, dass Lehrkräfte mit transmissiven Überzeugungen die Erziehungsziele in Ordnung und Disziplin sowie in der Vermittlung solider Kenntnisse sehen. Die zweite Fragebogenstudie (Studie 3) bestätigte die Ergebnisse von Chan und Elliott (2004) und Cheng et al. (2009), dass eine transmissive Lehr-Lern-Überzeugung mit naiven epistemologischen Überzeugungen zusammenhängt und eine konstruktivistische Überzeugung mit fortgeschrittenen epistemologischen Überzeugungen. Die Verbindung von Konstruktion und einem Lernbegriff als Verstehen sowie einem lernbegleitungsorientierten Lehrbegriff wurde in der ersten Fragebogenstudie (Studie 2) gezeigt, was wiederum das Ergebnis von Seidel et al. (2008) bestätigt.

Für den Zusammenhang der Lehr-Lern-Überzeugungen Partizipation und Schülerorientierung konnten im Vorfeld Erwartungen ausgehend von theoretischen Überlegungen formuliert werden, da diese Überzeugungen bisher nicht quantitativ untersucht worden waren. Mit der Erfassung der beiden Überzeugungen mithilfe des neuen Fragebogens Q4TB wurde zum ersten Mal versucht, diese Frage zu klären. In der ersten Fragebogenstudie (Studie 2) konnten Hinweise auf einen Zusammenhang zwischen Partizipation und 
Schülerorientierung und dem Lernbegriff „Lernen als Verstehen“ sowie dem lernbegleitungsorientierten Lehrbegriff gefunden werden. Daher wurde in der dritten Studie ein Zusammenhang zwischen der Lehr-Lern-Überzeugung Partizipation und fortgeschrittenen epistemologischen Überzeugungen erwartet. Diese Erwartung war zudem theoretisch begründet. Die Überzeugung Partizipation findet ihren theoretischen Hintergrund im Lerngemeinschaftsansatz (Lave \& Wenger, 1991) wieder. In diesem Ansatz wird das Wissen als gemeinschaftliches Gut, welches von den Mitgliedern einer (Lern-)Gemeinschaft konstruiert bzw. ausgehandelt wird, verstanden. Daher lag die Vermutung nahe, dass die Überzeugung Partizipation positiv mit fortgeschrittenen epistemologischen Überzeugungen zusammenhängt, die zum Beispiel das Wissen als komplex und unsicher verstehen sowie statt einer Expertengläubigkeit eine kritische Einstellung gegenüber der Expertenmeinung aufweisen.

Entgegen den Erwartungen konnte kein Zusammenhang zwischen Schülerorientierung sowie zwischen Partizipation und den epistemologischen Überzeugungen identifiziert werden. Eine mögliche Erklärung bei der Überzeugung Schülerorientierung kann in den Inhalten dieser Schülerorientierung gesehen werden- diese richtet nämlich den Fokus auf die Schülerinnen und Schüler. Diese Betonung der Schülerseite scheint konzeptuell unabhängig von Epistemologien zu sein, sodass die Schülerorientierung eher nicht mit der Vorstellung vom Wissen zusammenhängt. Bei der Überzeugung Partizipation kann es möglich sein, dass in ihrer Konzipierung, so wie sie in dem verwendeten Fragebogen getroffen wurde, die Idee der Wissenskonstruktion innerhalb einer Lerngemeinschaft nicht genügend Aufmerksamkeit bekommen hat und sich die partizipatorische Überzeugung eher über Ideen der Gemeinschaft mit deren Identifikationsmotiven und der gegenseitigen Unterstützungsmöglichkeit darstellt, so dass diese Darstellung der partizipatorischen Überzeugung nur wenige Berührungspunkte mit der Wissensvorstellung hat und dadurch kein Zusammenhang zwischen Partizipation und epistemologischen Überzeugungen identifiziert werden konnte. Es können allerdings auch Erklärungen für die nicht identifizierten Zusammenhänge zwischen epistemologischen Überzeugungen und der Überzeugung Partizipation sowie der Schülerorientierung vermutet werden, die im Untersuchungsdesign begründet liegen können. Da die Stichprobe mit $N=110$ Probanden relativ klein war, konnten die weniger starken Korrelationen zwischen epistemologischen Überzeugungen und den Überzeugungen Partizipation und Schülerorientierung möglicherweise nicht signifikant werden.

Mit dem Ergebnis des fehlenden Zusammenhangs zwischen epistemologischen Überzeugungen und Partizipation sowie Schülerorientierung kann die Annahme der 
„Überzeugungssyndrome“, wie sie in der COACTIV-Studie präsentiert werden, widerlegt werden. Es ist also nicht der Fall, dass die Überzeugungen zum Lehren und Lernen und die epistemologischen Überzeugungen in charakteristischen Mustern zusammenfallen. Gerade die Überzeugungen Partizipation und Schülerorientierung zeichneten sich durch fehlende Zusammenhänge zu epistemologischen Überzeugungen aus. Daher sollte für die zukünftige Forschung eine integrative Betrachtung der epistemologischen und lehr-/lernbezogenen Überzeugungen, wie sie in der COACTIV-Studie durchgehalten wird, abgelegt und eine separate Konzeptualisierung der Begriffe sowie entsprechende Untersuchungsdesigns angestrebt werden. Trotz der empirisch demonstrierten Beziehung zwischen epistemologischen Überzeugungen und den Überzeugungen Transmission und Konstruktion kann bei den Überzeugungen Partizipation und Schülerorientierung die Annahme getroffen werden, dass diese beiden Überzeugung inhaltlich unabhängig von den Vorstellungen von Wissen sind und sich auf andere wissens- und lerninhaltsunabhängige Aspekte beziehen.

\subsection{Grenzen der Arbeit und Ansätze für die weitere Forschung}

Abschließend soll auf einige Grenzen der vorliegenden Arbeit hingewiesen werden. Zum einen erfolgte die Konzipierung der vier Lehr-Lern-Überzeugungen deduktiv. Zwar wurden die Interviews als Hilfe bei der Bildung von den Facetten der Überzeugungen verwendet, jedoch wurden die zentralen Inhalte der Überzeugungen auf der Basis der bisherigen Literatur bestimmt. Somit konnten in der vorliegenden Arbeit nur die Überzeugungen untersucht werden, die in der bisherigen Literatur bekannt waren bzw. erwähnt wurden. Es war somit nicht möglich, neue Überzeugungen zu untersuchen, was jedoch auch nicht der Zielsetzung der vorliegenden Arbeit entsprach. Für zukünftige Studien mit dem Vorhaben, neuartige, noch nicht untersuchte Konzeptionen zu analysieren, wäre eine induktive Vorgehensweise bei der Interviewauswertung notwendig.

Ein möglicher Kritikpunkt betrifft die Auswertungsmethode der Interviews. Die Zuordnung der Einzelaussagen zu den Kategorien erfolgte durch zwei (trainierte) Auswerter, was ein Reliabilitätsproblem darstellen kann. Diesem Problem wurde entgegengewirkt, indem die beiden Auswerter ein detailliertes Segmentierungs- und Kodierungssystem mit Anweisungen und Ankerbeispielen hatten. Die durchgeführten Interrater-Reliabilitäten zeigten eine gute Übereinstimmung der beiden Auswerter. Allerdings erfolgte die Entscheidung darüber, inwieweit eine Kategorie einer Überzeugung zugeordnet werden kann, auf der theoretischen Basis. An diesem Punkt wäre eine zusätzliche Maßnahme denkbar, um 
die Validität der Untersuchung zu erhöhen. Denkbar wäre es auch, Experten nach ihrer Meinung bezüglich des erarbeiteten Kategoriensystems zu befragen. Im Allgemeinen wäre es für die zukünftige qualitative Forschung vorteilhaft, mehrere Untersucher in den Auswertungsprozess einzubeziehen und unter anderem auch Experten im jeweiligen Themengebiet zu befragen.

Des Weiteren ist an dieser Stelle die Stichprobe der Studien zu kritisieren. Zwar nahmen an der Interviewstudie neben den Lehramtsstudierenden auch Referendare und erfahrene Lehrkräfte teil, jedoch wurden im Rahmen der Fragebogenstudien keine Lehrkräfte im Beruf befragt. Um Aussagen über Lehrerüberzeugungen $\mathrm{zu}$ machen, müsste diese Personengruppe noch verstärkt in die Studien eingeplant werden. Dies war im Rahmen der vorliegenden Studie leider nicht möglich. Insgesamt fällt auf, dass in vielen Studien aus ökonomischen Gründen Lehramtsstudierende befragt werden. Diese Gruppe erreicht man meist einfacher als Personengruppen aus dem Schulkontext, wie zum Beispiel Lehrkräfte im Beruf. Gleichwohl wäre für die zukünftige Forschung wünschenswert, mehr Lehrkräfte im Beruf zu erreichen.

Die folgende kritische Anmerkung betrifft ebenfalls die Stichprobe. Die Interviewergebnisse zeigten, dass sich Lehr-Lern-Überzeugungen in Abhängigkeit vom Zeitpunkt in der Lehrerausbildung und vom Zeitpunkt in der Lehrerlaufbahn unterscheiden. Es wurden verschiedene Verläufe der vier Überzeugungen demonstriert. Allerdings basierten die Ergebnisse von zwei der drei Studien auf Untersuchungen mit Studierenden, da im Rahmen der Fragebogenstudien keine Lehrkräfte befragt wurden. Weiterhin war die Größe der Studierendengruppen zu unterschiedlichen Studienzeitpunkten recht unterschiedlich: Bachelorstudierende $=145$, Masterstudierende $=50$, Studierende am Anfang des Studiums $=$ 62, in der Mitte des Studiums $=17$ und am Ende des Studiums $=27$. Für zukünftige Forschung scheint deshalb eine größer angelegte Befragung notwendig, die sowohl gleich große Gruppen der Studierenden zu unterschiedlichen Zeitpunkten ihres Studiums, als auch mehr Lehrkräfte erreicht. Mit einer höheren Anzahl der Probanden in den Gruppen könnte eine bessere Repräsentativität erreicht werden. Dieser Kritik kann auch die Größe der Untergruppen in Bezug auf die Fächerkombination unterzogen werden. In der zweiten Studie wurden 15 Studierende der Naturwissenschaften, 116 Studierende der Geisteswissenschaften sowie 48 Studierende beider Fächer befragt. Auch in der dritten Studie war die Gruppe der Studierenden der Naturwissenschaften unterrepräsentiert: Naturwissenschaften $=10$, Geisteswissenschaften $=78$ Studierende und 22 Studierende mit beiden Fächern. Somit wäre 
auch unter diesem Gesichtspunkt eine größer angelegte Studie wünschenswert, um die Fragen nach den fächerkombinationsspezifischen Unterschieden zu klären.

Weiterhin ist zum Studiendesign anzumerken, dass alle drei Studien Querschnittsvergleiche darstellen. Die Ergebnisse dieser Studien können streng genommen nicht als Entwicklung der Überzeugungen interpretiert werden, sondern geben lediglich Hinweise auf diese. Um Entwicklungen der Lehr-Lern-Überzeugungen analysieren zu können, wären Längsschnittstudien notwendig, welche Lehrkräfte vom Anfang ihrer Lehrerausbildung über die Zeit des Studiums und die Referendariatszeit hinaus bis in die Berufsjahre hinein begleiten.

Ein weiterer Kritikpunkt betrifft den in der dritten Studie eingesetzten Fragebogen nach Schommer zur Messung epistemologischer Überzeugungen. Dieser Fragebogen zeigte gerade bei den lernspezifischen Skalen - Lernfähigkeit und Lerngeschwindigkeit - keine zufriedenstellenden Reliabilitätswerte ( $\alpha=.40$ bzw. $\alpha=.25$ ). Der Fragebogen wurde bereits in vielen Untersuchungen eingesetzt, allerdings wurde seine faktorielle Struktur nicht selten kritisiert (z.B. Hofer, 2002). In einigen Studien wurde eine alternative faktorielle Lösung erzielt, wie beispielweise in der Studie von Quian und Alvermann (1995), in der lediglich zwei der fünf Faktoren - Lernfähigkeit und Lerngeschwindigkeit - bestätigt wurden und zwei Faktoren zu einem gemeinsamen Faktor zusammenfielen (Struktur und Sicherheit des Wissens). Für die zukünftige Forschung wäre eine Wiederholung der Studie mit dem Einsatz eines zusätzlichen Instruments zur Messung von epistemologischen Überzeugungen wünschenswert.

Eine Stärke der vorliegenden Arbeit liegt in der Verbindung qualitativer und quantitativer Methoden. In der ersten Studie wurden die neu zu untersuchenden Lehr-LernÜberzeugungen mithilfe von Interviews mit angehenden und erfahrenen Lehrkräften analysiert, um festzustellen, ob diese Konstrukte in den Lehrerüberzeugungen existieren, ob die interviewten Lehrkräfte von sich aus Inhalte aller dieser Konstrukte äußern. Zudem wurden diese Interviewdaten nach der Methode von Chi (1998) quantifiziert, um für den Gruppenvergleich statistische Tests (z.B. die Varianzanalyse) anwenden zu können. Die Ergebnisse der Interviewstudie bestätigten die Annahme, dass in den Lehreransichten alle vier Lehr-Lern-Überzeugungen präsent sind. So konnte die Entwicklung des neuen Fragebogens nicht nur aus theoretischen Überlegungen legitimiert werden.

Zudem geht das Design der Interviewstudie mit den fünf Zeitpunkten der Lehrerausbildung und Berufslaufbahn über bisherige Studien hinaus, da diese nur zwei Gruppen von Lehrkräften miteinander verglichen (z.B. Martínez et al., 2001). So konnten in 
der vorliegenden Arbeit Unterschiede in den Lehr-Lern-Überzeugungen auf drei Zeitpunkte in der Lehrerausbildung sowie auf die Referendariatszeit und die Zeit im Lehrerberuf mit mindestens fünf Jahren Lehrerfahrung, bezogen werden. Eine Längsschnittstudie wäre indessen für die zukünftige Forschung notwendig. Diese könnte präzisere Aussagen über die Entwicklung von Lehrerüberzeugungen formulieren. 


\section{$8 \quad$ Zusammenfassung}

Lehrerüberzeugungen zum Lehren und Lernen wird eine Bedeutung für das unterrichtliche Handeln von Lehrkräften und für die Schülerleistung zugeschrieben (vgl. Dubberke et al., 2008; Fennema et al., 1990; Hartinger et al., 2006; Köller et al., 2000; Schönfeld, 1983, 2000; Stipek et al., 2001). Die empirische Forschung lieferte bereits Hinweise darauf, dass transmissive Lehr-Lern-Überzeugungen von Lehrkräften weniger positive Effekte auf die Schülerleistung im Fach Mathematik haben als konstruktivistische (Dubberke et al., 2011; Peterson et al., 1989; Staub \& Stern, 2002). Da sich die quantitative Forschung bisher ausschließlich auf das Überzeugungspaar Transmission vs. Konstruktion fokussierte (z.B. Kunter et al., 2011; Peterson et al., 1989; Staub \& Stern, 2002; OECD, 2009), wurden andere Sichtweisen auf das Lehren und Lernen vernachlässigt. Daher sollten in der vorliegenden Arbeit neben den Überzeugungen Transmission und Konstruktion auch partizipatorische und schülerorientierte Lehr-Lern-Überzeugungen untersucht werden.

Im ersten Schritt wurden bei der Analyse der einschlägigen Literatur verschiedene Überzeugungen identifiziert und einander gegenübergestellt. Für weitere Analysen wurden die vier Lehr-Lern-Überzeugungen - Transmission, Konstruktion, Partizipation und Schülerorientierung - ausgewählt, die unter ähnlichen Beschreibungen und Bezeichnungen in fast allen vorgefundenen Studien präsent waren. So konnte angenommen werden, dass neben Transmission und Konstruktion auch partizipatorische und schülerorientierte Überzeugungen in der Selbstauskunft angehender und berufserfahrener Lehrkräfte zum Lehren und Lernen identifiziert werden können. Da sowohl Partizipation als auch Schülerorientierung bisher fast ausschließlich qualitativ untersucht wurden, gab es dementsprechend bis dato keine Messinstrumente und keine Studien, die die Bedeutung dieser Überzeugungen für Lehrkräfte $\mathrm{zu}$ unterschiedlichen Zeitpunkten ihrer beruflichen Karriere untersucht haben. Daher stellte sich die Frage, inwieweit sich die vier Überzeugungen - Transmission, Konstruktion, Partizipation und Schülerorientierung - mithilfe eines quantitativen Messinstruments reliabel und valide messen lassen.

Die bisherige Forschung zur Entwicklung von Lehrerüberzeugungen berichtete von Unterschieden in den Lehr-Lern-Überzeugungen sowohl in Abhängigkeit vom Zeitpunkt in der Lehrerausbildung als auch vom Zeitpunkt in der Lehrerlaufbahn. Lehrerüberzeugungen am Anfang ihrer Ausbildung wurden als transmissiv und schülerorientiert beschrieben (Black \& Ammon, 1992; Brookhart \& Freeman, 1992; Calderhead, 1996; Richardson, 1996; Russel, 1988). Im Verlauf des Studiums schienen sich diese in Richtung konstruktivistischer 
Einsichten zu entwickeln (Hollingsworth, 1989; Nettle, 1998). Allerdings basierten diese Erkenntnisse auf qualitativen Untersuchungen, die einen oder zwei Zeitpunkte in der Lehrerlaufbahn beleuchteten. Daher bestand das Forschungsdesiderat in der Untersuchung von Lehrerüberzeugungen $\mathrm{zu}$ mehreren Zeitpunkten der Lehrerausbildung und Lehrerlaufbahn.

Sowohl epistemologische Überzeugungen als auch Überzeugungen zum Lehren und Lernen werden als Facetten professioneller Handlungskompetenz von Lehrkräften aufgefasst (Baumert \& Kunter, 2006) und als wichtige Aspekte für das Lehrerhandeln erachtet (Hashweh, 1985, 1996; Maggioni \& Parkinson, 2008; Olafson \& Schraw, 2006; Schraw \& Olafson, 2008). Die empirische Forschung zum Zusammenhang beider Konstrukte bestätigte einerseits die Annahme, dass naive epistemologische Überzeugungen wie Glauben an eine angeborene Lernfähigkeit oder an die Einfachheit des Wissens mit der traditionellen LehrLern-Vorstellung zusammenhängen und fortgeschrittene epistemologische Überzeugungen wie Lernen als Prozess zu konstruktivistischen Vorstellungen von Lehren und Lernen führen (Chan \& Elliott, 2004; Cheng et al., 2009). Allerdings wurden auch gegensätzliche Zusammenhänge vorgefunden (Cheng et al., 2009). Da weder für Partizipation noch für Schülerorientierung ein quantitatives Messinstrument vorlag, konnte deren Beziehung zu epistemologischen Überzeugungen bisher nicht untersucht werden. Die Untersuchung dieser Beziehung stellte also ein weiteres Forschungsdesiderat dar, welchem in der vorliegenden Arbeit nachgegangen wurde.

Zur Beantwortung dieser Fragen wurden Daten mithilfe von qualitativen und quantitativen Methoden erhoben: Zum einen wurden Lehramtsstudierende, Referendare und berufserfahrene Lehrkräfte $\mathrm{zu}$ ihren Lehr-Lern-Überzeugungen interviewt. Die inhaltsanalytisch ausgewerteten Daten wurden nach der Methode von Chi (1998) quantifiziert, sodass die fünf Gruppen mithilfe quantitativer Auswertungsmethoden (z.B. mehrfaktorielle Varianzanalysen) miteinander verglichen werden konnten. Zum anderen wurden Lehramtsstudierende an zwei deutschen Universitäten mit dem auf der Grundlage der Interviews sowie in Anlehnung an existierende Instrumente entwickelten Fragebogen zur Messung der vier Lehr-Lern-Überzeugungen befragt. In allen drei Studien wurden Lehr-LernÜberzeugungen von Studierenden zu unterschiedlichen Zeitpunkten in der Lehrerausbildung miteinander verglichen. In der Interviewstudie konnten zudem Lehr-Lern-Überzeugungen von Referendaren und erfahrenen Lehrkräften ananlysiert werden.

Insgesamt sprechen die Befunde aus der Interviewstudie (Studie 1) dafür, dass Lehrkräfte in ihren Ansichten zum Lehren und Lernen neben den Überzeugungen 
Transmission und Konstruktion auch partizipative und schülerorientierte Ideen haben. Sie trafen dennoch mehr auf Transmission bezogene Aussagen, sodass, verglichen mit den übrigen drei Überzeugungen, diese traditionelle Überzeugung insgesamt die am meisten verbreitete Überzeugung in der untersuchten Stichprobe war. In Abhängigkeit vom Zeitpunkt des Studiums (drei Zeitpunkte) sowie der Lehrerlaufbahn (Referendariat, 5 Jahre Lehrerfahrung) zeigten sich unterschiedliche Muster vor allem bei den Überzeugungen Konstruktion und Schülerorientierung. Anders als Schülerorientierung, welche im Verlauf des Studiums ihre Präferenz bei den zukünftigen Lehrkräften verlor, gewann Konstruktion im Verlauf des Studiums an Beliebtheit, verlor diese jedoch wieder mit dem Einstieg in den Beruf, während Schülerorientierung im Beruf wieder bedeutender wurde. Zudem schienen die Überzeugungsmuster erfahrener Lehrkräfte denen von Lehramtsstudierenden am Anfang des Studiums ähnlich zu sein. Beide zeigten niedrigere Werte in Konstruktion und Partizipation sowie hohe Werte in Transmission und Schülerorientierung. Da dieses Ergebnis mit der qualitativen Erhebungsmethode (Interviewstudie) zustande kam, wurde in der zweiten und der dritten Studie eine Replikation dieses Ergebnisses mithilfe einer quantitativen Erhebungsmethode angestrebt. Dafür war die Entwicklung eines neuen Instrumentes zur Erfassung der vier Lehr-Lern-Überzeugungen notwendig.

Um die Frage der Reliabilität und Konstruktvalidität des neu entwickelten Fragebogens zu beantworten, wurden die Daten zu Lehr-Lern-Überzeugungen von Lehramtsstudierenden an zwei deutschen Universitäten mit dem Fragebogen erhoben. Die Ergebnisse der exploratorischen Faktorenanalysen bestätigten die theoretisch postulierte 4-FaktorenStruktur mit den Faktoren Transmission, Konstruktion, Partizipation und Schülerorientierung. Die Skalen zeigten gute Reliabilitäten. Insgesamt konnten die Lehr-Lern-Überzeugungen bei Lehramtsstudierenden reliabel und valide gemessen werden. Zudem deuteten die Ergebnisse auf eine gute Konstruktvalidität des eingesetzten Instruments und auf eine ausreichende Sensitivität gegenüber geschlechts- und fächerkombinationsspezifischen Unterschieden in den Überzeugungen. Die Frage nach der Beziehung der vier Überzeugungen Transmission, Konstruktion, Partizipation und Schülerorientierung zueinander verdeutlichte, dass sich die Überzeugung Transmission von den übrigen drei absetzte und Konstruktion, Partizipation und Schülerorientierung zueinander enger standen als zu Transmission.

In allen drei Studien stand die Frage nach der Bedeutung der Überzeugungen zu verschiedenen Zeitpunkten in der Lehrerausbildung und Lehrerlaufbahn im Zentrum. Wie bereits geschildert, wurden in der ersten Studie die Lehr-Lern-Überzeugungen von Lehramtsstudierenden zu drei Zeitpunkten miteinander verglichen: am Anfang $(n=10)$, in der 
Mitte $(n=10)$ und am Ende ihres Lehramtsstudiums ( $n=10)$, sowie Referendare $(n=10)$ und berufserfahrene Lehrkräfte $(n=10)$. In der zweiten Studie wurden Überzeugungen von Bachelorstudierenden $(n=145)$ mit den Überzeugungen von Masterstudierenden $(n=50)$ verglichen. In der dritten Studie wurden die Überzeugungen von Lehramtsstudierenden am Anfang ihres Studiums $(n=62)$, mit denen in der Mitte $(n=17)$ und am Ende ihres Studiums $(n=27)$ verglichen. Insgesamt demonstrierten die Ergebnisse der beiden Fragebogen-Studien, dass die Überzeugungen Konstruktion und Partizipation im Verlauf des Studiums an Beliebtheit gewannen. Im Vergleich zu Bachelorstudierenden (Studie 2), die in der Überzeugung Transmission hohe Werte erzielten, zeigten Masterstudierende höhere Werte in der Überzeugung Partizipation. In der dritten Studie erzielten die Studierenden am Ende des Studiums signifikant höhere Werte in der Überzeugung Konstruktion. Durchgeführte Regressionsanalysen belegten zudem, dass mit steigender Semesteranzahl die Werte in Konstruktion und Partizipation anstiegen. In der Interview-Studie zeigten sich ähnliche Muster in Konstruktion im Verlauf des Studiums sowie gegensätzliche Entwicklungen der Überzeugung Schülerorientierung. Allerdings wurden schülerorientierte Überzeugungen im Beruf wieder wichtiger, während Konstruktion an Bedeutung verlor. Insgesamt geben die Ergebnisse der drei Studien Hinweise darauf, dass sich die Lehr-Lern-Überzeugungen sowohl in Abhängigkeit vom Zeitpunkt in der Lehrerausbildung als auch vom Zeitpunkt in der Lehrerlaufbahn verändern: Die Überzeugungen Konstruktion und Partizipation gewinnen im Verlauf der Lehrerausbildung an Präferenz und die Überzeugungen Transmission und Schülerorientierung finden wieder mehr Zuspruch mit dem Einstieg in den Lehrerberuf.

In Studie 3 stand die Frage nach dem Zusammenhang zwischen epistemologischen und lehr-/lernbezogenen Überzeugungen im Zentrum. Hierfür wurden mithilfe der Skalen des „Epistemological Beliefs Questionnaire“ (EBQ) (Schommer, 1990, 1992) epistemologische Überzeugungen und mithilfe des „Fragebogens zur Messung der vier Lehrerüberzeugungen zum Lehren und Lernen“ (The Questionnaire of Four Teachers' Beliefs - Q4TB) (Schlichter et al., in Vorbereitung) die Überzeugungen Transmission, Konstruktion, Partizipation und Schülerorientierung erhoben. Insgesamt deuten die Ergebnisse der Studie 3 darauf hin, dass eine hohe Zustimmung zu Konstruktion mit elaborierteren epistemologischen Überzeugungen und eine hohe Zustimmung zur Transmission mit naiven epistemologischen Überzeugungen einhergingen. Des Weiteren schien die Überzeugung Schülerorientierung nicht mit epistemologischen Überzeugungen zusammenzuhängen, was die theoretische Annahme bestätigte. Anders als erwartet zeigte sich auch zwischen den epistemologischen Überzeugungen und der Partizipation eine Null-Korrelation. 
Zusammenfassend lässt sich festhalten, dass im Rahmen der vorliegenden Arbeit zum ersten Mal über die zwei Lehr-Lern-Überzeugungen Transmission und Konstruktion hinaus die Überzeugungen Partizipation und Schülerorientierung mit qualitativen und quantitativen Methoden untersucht wurden und verschiedene Muster in diesen Lehr-Lern-Überzeugungen $\mathrm{zu}$ unterschiedlichen Zeitpunkten sowohl während der Lehrerausbildung als auch der Lehrerlaufbahn aufgezeigt werden konnten. Der zentrale Ertrag der Arbeit stellt die Entwicklung des Fragebogens zur Messung der vier Lehr-Lern-Überzeugungen dar, mit dessen Hilfe sich Transmission und Konstruktion, aber auch Partizipation und Schülerorientierung reliabel und valide messen lassen. Dieser Fragebogen kann in der zukünftigen Forschung zur Klärung der Fragen nach der Bedeutung der vier Überzeugungen für schulische Lernkontexte, wie beispielsweise für das Lehrerhandeln und Schülerlernen, beitragen. Insbesondere kann der Q4TB in der beruflichen Praxis zu diagnostischen Zwecken eingesetzt werden. Mit seiner Hilfe können Lehr-Lern-Überzeugungen von Lehrkräften ökonomisch und zeitsparend bestimmt werden. Mit der Kenntnis eigener Lehr-LernÜberzeugungen können Lehrkräfte Rückschlüsse auf ihren Unterricht ziehen und über die eigene Unterrichtspraxis sowie die Beziehung $\mathrm{zu}$ ihren Schülerinnen und Schülern reflektieren. 


\section{Literaturverzeichnis}

Aebli, H. (1983). Zwölf Grundformen des Lehrens: Eine allgemeine Didaktik auf psychologischer Grundlage. Stuttgart: Klett.

Aguirre, J., \& Speer, N. M. (2000). Examining the relationship between beliefs and goals in teacher practice. Journal of Mathematical Behavior, 18(3), 327-356.

Alexander, P. A., \& Dochy, F. (1995). Conceptions of knowledge and beliefs: A comparison across varying cultural and educational communities. American Educational Research Journal, 32(2), 413-442.

Alger, C. L. (2009). Secondary teachers' conceptual metaphors of teaching and learning: Changes over the career span. Teaching and Teacher Education, 25, 743-751.

Ames, C., \& Archer, J. (1988). Achievement goals in the classroom: Students' learning strategies and motivation processes. Journal of Educational Psychology, 80(3), 260267.

Anderman, E., \& Young, A. (1994). Motivation and strategy use in science: Individual differences and classroom effects. Journal of Research in Science Teaching, 31, 811831.

Anderson, J., Reder, L. M., \& Simon, H. A. (1997). Situative versus cognitive perspectives: Form versus substance. Educational Researcher, 26(1), 18-21.

Assor, A., Kaplan, H., \& Roth, G. (2002). Choice is good, but relevance is excellent: Autonomy-enhancing and suppressing teacher behaviors predicting students' engagement in schoolwork. British Journal of Educational Psychology, 72(2), 261278 .

Bandura, A. (1986). Social foundations of thought and action: A social cognitive theory. Engelwood Cliffs, NJ: Prentice-Hall.

Bauer, J., Drechsel, B., Möller, J., Prenzel, M., Retelsdorf, J., Rösler, L. et al. (2009). Skalen und Items des BMBF-Projektes: PaLea - Panel zum Lehramtsstudium. Skalen und Items Bachelorfragebogen 1. Welle.

Bauer, J., Drechsel, B., Retelsdorf, J., Sporer, T., Rösler, L., Prenzel, M. et al. (2010). Panel zum Lehramtsstudium - PaLea: Entwicklungsverläufe zukünftiger Lehrkräfte im Kontext der Reform der Lehrerbildung. Beiträge zur Hochschulforschung, 32, 34-55.

Baumert, J, Blum, W., Brunner, M., Dubberke, T., Jordan, A., Klusmann, U. et al. (2008). Professionswissen von Lehrkräften, kognitiv aktivierender Mathematikunterricht und die Entwicklung von mathematischer Kompetenz (COACTIV): Dokumentation der 
Erhebungsinstrumente. Nr. 83 Materialien aus der Bildungsforschung. Berlin: MaxPlank-Institut für Bildungsforschung.

Baumert, J, Bos, W., Brockmann, J., Gruehn, S., Klieme, E., Köller, O. et al. (2000). TIMMS III Deutschland. Der Abschlussbericht. Zusammenfassung ausgewählter Ergebnisse der Dritten Internationalen Mathematik- und Naturwissenschaftsstudie zur mathematischen und naturwissenschaftlichen Bildung am Ende der Schullaufbahn. Berlin.

Baumert, J., \& Kunter, M. (2006). Stichwort: Professionelle Kompetenz von Lehrkräften. Zeitschrift für Erziehungswissenschaft, 9(4), 469-520.

Belenky, M. F., Clinchy, B. M., Goldberger, N. R., \& Tarule, J. M. (1986). Women's ways of knowing: The development of the self, voice, and mind. New York: Basic Books.

Bell, P., \& Linn, M. C. (2002). In B. K. Hofer, \& P. R. Pintrich (Eds.), Personal epistemology: The psychology of beliefs about knowledge and knowing (pp. 321-346). Mahwah, NJ: Erlbaum.

Benson, G. D. (1989). Epistemology and science curriculum. Journal of Curriculum Studies, $21,329-344$.

Berliner, D. C. (1988). The development of expertise in pedagogy. Paper presented at the meeting of the American Association of Colleges for Teacher Education, New Orleans, LA.

Black, A., \& Ammon, P. (1992). A developmental-constructivist approach to teacher education. Journal of Teacher Education, 44(1), 27-37.

Borko, H., \& Livingston, C. (1989). Cognition and improvisation: Differences in mathematics instruction by expert and novice teachers. American Educational Research Journal, 26(4), 473-498.

Borko, H., \& Putnam, R. T. (1996). Learning to teach. In D. C. Berliner \& R. C. Calfee (Eds.), Handbook of educational psychology (pp. 673-708). New York: Macmillan.

Bortz, J. (2005). Statistik für Human- und Sozialwissenschaftler (6., vollständig überarbeitete und aktualisierte Aufl.). Berlin: Springer.

Boulton-Lewis, G. M., Smith, D. J. H., McCrindle, A. R., Burnett, P. C., \& Campbell, K. J. (2001). Secondary teachers' conceptions of teaching and learning. Learning and Instruction, 11, 35-51.

Bransford, J., Darling-Hammond, L., \& LePage, P. (2005). Introduction. In L. DarlingHammond, \& J. Bransford (Eds.), Preparing teachers for a changing world (pp. 139). San Francisco: CA: Jossey-Bass. 
Braten, I., \& Stromso, H. I. (2005). The relationship between epistemological beliefs, implicit theories of intelligence, and self-regulated learning among Norwegian postsecondary students. British Journal of Educational Psychology, 75, 539-565.

Bereiter, C. (1994). Constructivism, socioculturalism, and Popper's World 3. Educational Researcher, 23(7), 13-20.

Bielaczyc, K., \& Collins, A. (1983). Learning communities in classrooms: A reconceptualization of educational practice. In C. M. Reigeluth (Ed.), Instructional design theories and models. (Vol. II, pp. 269-292). Mahwah, NJ: Lawrence Erlbaum Associates.

Broekkamp, H., \& van Hout-Wolters, B. (2007). The gap between educational research and practice: A literature review, symposium, and questionnaire. Educational Research and Evaluation, 13(3), 203-220.

Bromme, R. (1981). Das Denken von Lehrern bei der Unterrichtsvorbereitung. Eine empirische Untersuchung von kognitiven Prozessen von Mathematiklehrern. Weinheim, Basel: Beltz.

Bromme, R. (1994). Beyond subject matter: A psychological topology of teachers' professional knowledge. In R. Biehler, R.W. Schnotz, R. Sträßer, \& B. Winkelmann (Eds.), Mathematics didactics as a scientific discipline: The state of the art (pp. 7788). Dordrecht: Kluwer.

Bromme, R. (1997). Kompetenzen, Funktionen und unterrichtliches Handeln des Lehrers. In F. E. Weinert (Hrsg.), Enzyklopädie der Psychologie: Pädagogische Psychologie, Bd. 3. Psychologie des Unterrichts und der Schule (S. 177-212). Göttingen.

Bromme, R., Klienhues, D., \& Stahl, E. (2008). Knowledge and epistemological beliefs: An intimate but complicate relationship. In M. S. Khiene (Ed.), Knowing, knowledge, and beliefs: Epistemological studies across diverse cultures (pp. 423-441). New York: Springer.

Bromme, R., \& Rheinberg, F. (2006). Lehrende in Schulen. In A. Krapp \& B. Weidenmann (Eds.), Pädagogische Psychologie (5. Aufl., S. 296-334). Weinheim: Beltz.

Brookhart, S. M., \& Freeman, D. J. (1992). Characteristics of entering teacher candidates. Review of Educational Research, 62, 37-60.

Brown, J. S., Collins, J. B., \& Duguid, P. (1989). Situated cognition and the culture of learning. Educational Researcher, 18(1), 32-42.

Brown, C. A., \& Cooney, T. J. (1982). Research on teacher education: A philosophical orientation. Journal of Research and Development in Education, 15(4), 13-18. 
Brunner, M., Kunter, M., Krauss, S., Klusmann, U., Baumert, J., Blum, W. et al. (2006). Die professionelle Kompetenz von Mathematiklehrkräften: Konzeptualisierung, Erfassung und Bedeutung für den Unterricht. Eine Zwischenbilanz des COACTIV-Projektes. In M. Prenzel \& L. Allolio-Näcke (Hrsg.), Untersuchungen zur Bildungsqualität der Schule. Abschlussbericht des DFG-Schwerpunktprogramms (S. 54-82). Münster: Waxmann.

Buehl, M. M., \& Alexander, P. A. (2001). Beliefs about academic knowledge. Educational Psychology Review, 13(4), 385-418.

Buehl, M. M., Alexander, P. A., \& Murphy, P. K. (2002). Beliefs about schooled knowledge: Domain specific or domain general? Contemporary Educational Psychology, 27, 415443.

Burkhardt, H., \& Schoenfeld, A. H. (2003). Improving educational research: Toward a more useful, more influential, and better-funded enterprise. Educational Research, 32(9), 314.

Calderhead, J. (1981). Stimulated Recall: A method for research on teaching. British Journal of Educational Psychology, 51, 211-217.

Calderhead, J., \& Robson, M. (1991). Images of teaching: Student teachers' early conceptions of classroom practice. Teaching and Teacher Education, 1(7), 1-8.

Calderhead, J. (1996). Beliefs and knowledge. In D. C. Berliner \& R. C. Calfee (Eds.), Handbook of educational psychology (pp. 709-725). New York: Macmillan.

Cano, F. (2005). Epistemological beliefs and approaches to learning: Their change through secondary school and their influence on academic performance. British Journal of Educational Psychology, 75(2), 203-221.

Chan, K.-W., \& Elliott, R. G. (2004). Relational analysis of personal epistemology and conceptions about teaching and learning. Teaching and Teacher Education, 20(8), 817-831.

Cheng, M. H., Chan, K.-W., Tang, S. Y., \& Cheng, A. Y. (2009). Pre-service teacher education students' epistemological beliefs and their conceptions of teaching. Teaching and Teacher Education, 25, 319-327.

Chi, M. T. (1997). Quantifying qualitative analyses of verbal data: A practical guide. The Journal of the Learning Sciences, 6(3), 271-315.

Chi, M. T., Glaser, R., \& Farr, M. (Eds.). (1988). The nature of expertise. Hillsdale, NJ: Erlbaum. 
Clark, C. M. (1988). Asking the right questions about teacher preparation: Contribution of research on teaching thinking. Educational Researcher, 17(2), 5-12.

Clark, C. M., \& Peterson, P. L. (1986). Teachers' thought processes. In M. C. Wittrock (Ed.), Handbook of research on teaching (3rd. ed., pp. 255-296). New York: Macmillan.

Clausen, M., Reusser, K., \& Klieme, E. (2003). Unterrichtsqualität auf der Basis hochinferenter Unterrichtsbeurteilungen. Ein Vergleich zwischen Deutschland und der deutschsprachigen Schweiz. Unterrichtswissenschaft, 31(2), 122-141.

Cobb, P., Wood, T., Yackel, E., Nicholls, J., Wheatley, G., Trigatti, B. et al. (1991). Assessment of a problem-centered second-grade mathematics project. Journal of Research in Mathematics Education, 22, 3-29.

Cochran-Smith, M., \& Zeichner, K. (Eds.). (2005). Studying teacher education. The report of the AERA panel on research and teacher education. Mahwach: Lawrence Erlbaum.

Cohen, D. (1990). A revolution in the classroom: The case of Mrs. Oublier. Education Evaluation and Policy Analysis, 12(3), 311-329.

Cole, A. L. (1989). Making explicit implicit theories of teaching: Starting points in preservice programs. Paper presented at the American Educational Research Association, San Francisco, CA.

Collins, A., Brown, J. S., \& Newman, S. E. (1989). Cognitive apprenticeship: Teaching the craft of reading, writing, and mathematics. In L. B. Resnick (Ed.), Knowing, learning, and instruction: Essays in honor of Robert Glaser (pp. 453-494). Hillsdale, NJ: Erlbaum.

Collins, J. B., Jarvis-Selinger, S., \& Pratt, D. D. (2002). How do perspectives on teaching vary across disciplinary majors for student enrolled in teacher preparation? Verfügbar unter: http://teachingperspectives.com/PDF/howdoteachers.pdf (10.08.2012).

Cooney, T. J. (1985). A beginning teacher's view of problem solving. Journal for Research in Mathematics Education, 16, 324-336.

Cunningham, J. W., \& Fitzgerald, J. (1996). Epistemology and reading. Reading Research Quarterly, 31, 36-60.

Davis, E. A. (1997). Students' epistemological beliefs about science and learning. Paper presented at the American Educational Research Association, Chicago, IL.

Dahl, T. I., Bals, M., \& Turi, A. L. (2005). Are students' beliefs about knowledge and learning associated with their reported use of learning strategies? British Journal of Educational Psychology, 75, 257-273. 
Dahlgren, M. A., \& Chiriac, E. H. (2009). Learning for professional life: Student teachers' and graduated teachers' views of learning, responsibility and collaboration. Teaching and Teacher Education, 25, 991-999.

Daniels, Z. (2008). Entwicklung schulischer Interessen im Jugendalter. In: D. Rost (2008): Pädagogische Psychologie und Entwicklungspsychologie (Bd. 69). Münster/New York: Waxmann.

Dann, H.-D., Cloetta, B., Müller-Fohrbrodt, G., \& Helmreich, R. (1978). Umweltbedingungen innovativer Kompetenz. Eine Längsschnittuntersuchung zur Sozialisation von Lehrern in Ausbildung und Beruf. Stuttgart: Klett-Cotta.

Dann, H.-D., Müller-Fohrbrodt, G., \& Cloetta, B. (1981). Sozialisation junger Lehrer im Beruf: „Praxisshock“ drei Jahre später. Zeitschrift für Entwicklungspsychologie und Pädagogische Psychologie, 13(3), 251-262.

Darling-Hammond, L., \& Bransford, J. (2005). Preparing teachers for a changing world. What teachers should learn and be able to do. San Francisco, CA: Jossey-Bass.

DeBacker, T. K., \& Crowson, H. M. (2006). Influences on cognitive engagement: Epistemological beliefs and need for closure. British Journal of Educational Psychology, 76, 535-551.

Deci, E. L., \& Ryan, R. M. (1985). Intrinsic motivation and self-determination in human behavior. New York: Plenum Press.

Deci, E. L., \& Ryan, R. M. (1987). The support of autonomy and the control of behavior. Journal of Personality and Social Psychology, 53, 1024-1037.

Deci, E. L., \& Ryan, R. M. (1993). Die Selbstbestimmungstheorie der Motivation und ihre Bedeutung für die Pädagogik. Zeitschrift für Pädagogik, 39, 223-228.

De Corte, E. (2004). Mainstreams and perspectives in research on learning (mathematics) from instruction. Applied Psychology, 53(2), 279-310.

Dewey, J. (1933). How to think. Boston: D.C. Heath.

Dubberke, T., Kunter, M., Mc Elvany, N., Brunner, M., \& Baumert, J. (2008). Lerntheoretische Überzeugungen von Mathematiklehrkräften: Einflüsse auf die Unterrichtsgestaltung und den Lernerfolg von Schülerinnen und Schülern. Zeitschrift für Pädagogische Psychologie, 22, 193-206.

Duell, O., \& Schommer-Aikins, M. (2001). Measures of people's beliefs about knowledge and learning. Educational Psychology Review, 13(4), 419-449.

Dunkin, M. J., \& Precians, R. P. (1992). Award-winning university teachers' concepts of teaching. Higher Education, 24, 483-502. 
Duschl, R. A. (1990). Restructuring science education. New York: Teachers College Press.

Dweck, C. S., \& Legget, E. L. (1988). A social-cognitive approach to motivation and personability. Psychological Review, 95, 256-273.

Elder, A. D. (2002). Characterizing fifth grade students' epistemological beliefs in science. In B. K. Hofer, \& P. R. Pintrich (Eds.), Personal epistemology: The psychology of beliefs about knowledge and knowing (pp. 347-363). Mahwah, NJ: Erlbaum.

Elliot, A. J. (1999). Approach and avoidance motivation and achievement goals. Educational Psychologist, 34(3), 169-189.

Ernest, P. (1989). The knowledge, beliefs, and attitudes of the mathematics teacher: A model. Journal of Education for Teaching, 15(1), 13-33.

Etchberger, M. L., \& Shaw, K. L. (1992). Teacher change as a progression of transitional image: A chronology of a developing constructivist teacher. School Science and Mathematics, 92, 411-417.

Fang, Z. (1996). A review of research on teacher beliefs and practices. Educational Research, $38(1), 47-65$.

Feiman-Nemser, S., \& Remillard, J. (1996). Perspectives on learning to teach. In F. B. Murray (Ed.), The teacher educator's handbook: Building a knowledge base for the preparation of teachers (pp. 63-91). San Francisco, CA: Jossey Bass.

Fend, H. (2006). Neue Theorie der Schule: Einführung in das Verstehen von Bildungssystemen (Bd. 1). Wiesbaden: VS Verlag für Sozialwissenschaften.

Fennema, E., Carpenter, T. P., \& Loef, M. (1990). Teacher belief scale: Cognitively guided instruction project. Madison: University of Wisconsin.

Fennema, E., \& Loef Franke, M. (1992). Teachers' knowledge and its impact. In D. A. Grouws (Ed.), Handbook of research on mathematics teaching and learning (pp. 147164). New York: Macmillan.

Fenstermacher, G. D. (1979). A philosophical consideration of recent research in teacher effectiveness. In L. S. Shulman (Ed.), Review of Research in Education $\left(6^{\text {th }}\right.$ ed., pp. 157-185). Itaska, IL: Peacock.

Fenstermacher, G. D. (1994). The knower and the known: The nature of knowledge in research on teaching. In L. Darling-Hammmond (Ed.), Review of Research in Education (Vol. 20, pp. 3-56). Waschington, DC: American Educational Research Association.

Fielstein, L., \& Phelps, P. (2001). Introduction to teaching: Rewards and realities. Belmont, CA: Wadsworth. 
Fischler, H. (2001). Verfahren zur Erfassung von Lehrer-Vorstellungen zum Lehren und Lernen in den Naturwissenschaften. Zeitschrift für Didaktik der Naturwissenschaften, $7,105-120$.

Garrett-Ingram, C. (1997). Something to believe in: The relationship between epistemological beliefs and study strategies. Paper presented at the American Educational Research Association, Chicago, IL.

Gill, M. G., Ashton, P. T., \& Algina, J. (2004). Beliefs about Schooled Knowledge: Domain General or Domain Specific? Contemporary Educational Psychology, 27, 415-443.

Ginsburg, H., \& Opper, S. (1991). Piagets Theorie der geistigen Entwicklung (6. Aufl.). Stuttgart: Klett-Cotta.

Greeno, J. G. (1997). On claims that answer the wrong questions. Educational Researcher, $26(1), 5-17$.

Greeno, J. G., Collins, A., \& Resnick, L. B. (1996). Cognition and learning. In D. C. Berliner \& R. C. Calfee (Eds.), Handbook of educational psychology (pp. 15-46). New York: Macmillan.

Grigutsch, S., Raatz, U., \& Törner, G. (1998). Einstellungen gegenüber Mathematik bei Mathematiklehrern. Journal für Mathematikdidaktik, 19(1), 3-45.

Gow, L., \& Kember, D. (1993). Conceptions of teaching and their relationship to student learning. British Journal of Educational Psychology, 63, 20-33.

Grossman, P. (2008). Responding to our critics: From crisis to opportunity in research on teacher education. Journal of Teacher Education, 59, 10-23.

Gruber, H., \& Renkl, A. (1995). Die Entwicklung epistemologischer Überzeugungen bei Studierenden. Beitrag zur 37. Tagung Experimentell Arbeitenden Psychologen in Bochum.

Gruehn, S. (2000). Unterricht und schulisches Lernen. Münster: Waxmann.

Hänsel, D. (1975). Die Anpassung des Lehrers . Zur Sozialisation in der Berufspraxis. Basel: Weinheim.

Hall, V. C., Chiarello, K. S., \& Edmondson, B. (1996). Deciding where knowledge comes from depends on where you look. Journal of Educational Psychology, 88, 305-313.

Hammer, D., \& Elby, A. (2003). Tapping epistemological resources for learning physics. Journal of the Learning Sciences, 12(1), 53-90.

Handal, B. (2003). Teacher's mathematical beliefs: A review. The Mathematic Educator, 13(2), 47-57. 
Hanke, U., \& Schwarz, L. (2011). Übersetzung des „Teaching Perspectives Inventory“ ins Deutsche und Validierung. Vortrag auf der Sektionstagung Empirische Bildungsforschung 2011 (AEPF), Bamberg.

Hartinger, A., Kleickmann, T., \& Hawelka, B. (2006). Der Einfluss von Lehrervorstellungen zum Lernen und Lehren auf die Gestaltung des Unterrichts und auf motivationale Schülervariablen. Zeitschrift der Erziehungswissenschaft, 9(1), 110-126.

Harvey, O. J. (1986). Beliefs systems and attitudes toward the death penalty and other punishments. Journal of Psychology, 54, 143-159.

Hashweh, M. Z. (1985). An exploratory study of teacher knowledge and teaching: The effects of science teachers' knowledge of subject-matter and their conceptions of learning on their teaching. Unpublished doctoral dissertation, Stanford University, California.

Hashweh, M. Z. (1996). Effects of science teacher's epistemological beliefs in teaching. Journal of Research in Science Teaching, 33(1), 47-63.

Hattie, J. (2009). Visible learning: A synthesis of over 800 meta-analyses relating to achievement. London: Routledge.

Helsper, W. (2004). Antinomien, Widersprüche, Paradoxien: Lehrerarbeit - ein unmögliches Geschäft? Eine strukturtheoretisch-rekonstruktive Perspektive auf das Lehrerhandeln. In B. Koch-Priewe, F.-U. Kolbe, \& J. Wildt (Hrsg.), Grundlagenforschung und mikrodidaktische Reformansätze zur Lehrerbildung (S. 49-98). Bad Heilbrunn.

Hofer, B. K., \& Pintrich, P. R. (1997). The development of epistemological theories: beliefs about knowledge and knowing and their relation to learning. Review of Educational Research, 67, 88-140.

Hofer, B. K. (2000). Dimensionality and disciplinary differences in personal epistemology. Contemporary Educational Psychology, 25, 378-405.

Hofer, B. K. (2001). Personal epistemology research: Implications for learning and teaching. Journal of Educational Psychology Review, 13(4), 353-383.

Hofer, B. K., \& Pintrich, P. R. (Eds.). (2002). Personal epistemology. Mahwah, NJ: Erlbaum

Hollingsworth, S. (1989). Prior beliefs and cognitive change in learning to teach. American Educational Research Journal, 26, 160-189.

Howard, B. C., McGee, S., Schwartz, N., \& Purcell, S. (2000). The experience of constructivism: Transforming teacher epistemology. Journal of Research on Computing in Education, 32(4), 455-465. 
Huitt, W. (2009). Humanism and Open Education. Educational Psychology Interactive. Valdosta, GA: Valdosta State University. Retrieved from http://www.edpsycinteractive.org/topics/affect/humed.html

Jehng, J. J., Johnson, S. D., \& Anderson, R. C. (1993). Schooling and students' epistemological beliefs about learning. Contemporary Educational Psychology, 18, 23-35.

Kagan, D. M. (1992). Implication of research on teacher belief. Educational Psychologist, 27(10), 65-90.

Kagan, D. M. (1992). Professional growth among beginning and pre-service teachers. Review of Educational Research, 62, 129-169.

Kardash, C. A. M., \& Scholes, R. J. (1996). Effects on preexisting beliefs, epistemological beliefs, and need for cognition on interpretation of controversial issues. Journal of Educational Psychology, 88(2), 260-271.

Kember, D. (1997). A reconceptualisation of the research into university academics' conceptions of teaching. Learning and Instruction, 7(3), 255-275.

Kember, D., \& Kwan, K. (2000). Lecturers' approaches to teaching and their relationship to conceptions of good teaching. Instructional Science, 28, 469-490.

Kitchener, R. F. (2002). Folk epistemology: An introduction. New Ideas in Psychology, 20 (pp. 89-105).

Kincheloe, J., Slattery, P., \& Steinberg, S. (2000). Contextualizing teaching. New York: Longman.

King, P. M., \& Kitchener, K. S. (1994). Developing reflective judgment: Understanding and Promoting Intellectual Growth and Critical Thinking in Adolescents and Adults. San Francisco, CA: Jossey-Bass.

Klafki, W. (1996). Neue Studien zur Bildungstheorie und Didaktik (5 Aufl.). Weinheim: Beltz.

Klauer, K. J., \& Leutner, D. (2007). Lehren und Lernen: Einführung in die Instruktionspsychologie. Weinheim: Beltz PVU.

Klieme, E., Lipowsky, F., Rakoczy, K., \& Ratzka, N. (2006). Qualitätsdimensionen und Wirksamkeit von Mathematikunterricht. In M. Prenzel, \& L. Allolio-Näcke (Hrsg.), Untersuchungen zur Bildungsqualität von Schule, Abschlussbericht des DFGSchwerpunktprogramms (S. 127-146). Münster: Waxmann.

KMK. (1977). Empfehlungen zur Arbeit in der gymnasialen Oberstufe. Bildung und Erziehung, 31 (S. 561-574). 
Koch, J. J. (1972). Lehrer-Studium und Beruf. Einstellungswandel in den ersten beiden Phasen der Ausbildung. Ulm: Süddeutsche Verlagsgesellschaft.

Korthagen, F. A. J. (2010). Situated learning theory and pedagogy of teacher education: Toward an integrative view of teacher behavior and teacher learning. Teaching and Teaching Education, 26, 96-106.

Köller, O., Baumert, J., \& Neubrand, J. (2000). Epistemologische Überzeugungen und Fachverständnis im Mathematik- und Physikunterricht. In J. Baumert, W. Bos \& R. H. Lehmann (Hrsg.), TIMSS III: Dritte internationale Mathematik- und Naturwissenschaftsstudie: Mathematische und naturwissenschaftliche Bildung am Ende der Schullaufbahn (Bd. 2): Mathematische und physikalische Kompetenzen am Ende der gymnasialen Oberstufe (S. 229-270). Opladen: Leske + Budrich.

Köller, O., Watermann, R., Trautwein, U., \& Lüdtke, O. (2004). Wege zu Hochschulreife in Baden-Württemberg. TOSCA - Eine Untersuchung an allgemein bildenden Schulen und beruflichen Gymnasien. Opladen: Leske + Budrich.

Kolbe, F.-U. (2004). Verhältnis von Wissen und Handeln. In S. Blömeke, P. Reinhold, G. Tulodziecki, \& J. Wirld (Hrsg.), Handbuch Lehrerbildung (S. 206-232). Bad Heilbrunn.

Krettenauer, T. (2005). Die Erfassung des Entwicklungsniveaus epistemologischer Überzeugungen und das Problem der Übertragbarkeit von Interviewverfahren in standardisierte Fragebogenmethoden. Zeitschrift für Entwicklungspsychologie und Pädagogische Psychologie, 37(2), 69-79.

Kuhn, D. (1999). Metacognitive development. In L. Balter \& C. S. Tamis-LeMonda (Eds.), Child psychology: A handbook of contemporary issues (pp. 259-286). Philadelphia: Psychology Press.

Kuhn, D., Cheney, R., \& Weinstock, M. (2000). The development of epistemological understanding. Cognitive Development, 15(3), 309-328

Kuhn, D., \& Park, S. H. (2005). Epistemological understanding and the development of intellectual values. International Journal of Educational Research, 43(3), 111-124.

Kunter, M., Klusmann, U., Dubberke, T., Baumert, J., Blum, W., \& Brunner, M. (2007). Linking aspects of teacher competence to their instruction. Results from the COACTIV project. In M. Prenzel (Ed.), Studies on the educational quality of schools. The final report on the DFG Priority Program (pp. 39-59). Münster: Waxmann. 
Kunter, M., Baumert, J., Blum, W., Klusmann, U., Krauss, S., \& Neubrand, M. (2011). Professionelle Kompetenz von Lehrkräften. Ergebnisse des Forschungsprogramms COACTIV. Münster: Waxmann.

Lakoff, G., \& Johnson, W. (1980). Metaphors we live by. Chicago: University of Chicago Press.

Lampert, M. (1990). When the problem is not the question and the solution is not the answer: Mathematical knowing and teaching. American Educational Research Journal, 27, 29-63.

Law, Y.-K., Chan, C. K. K., \& Sachs, J. (2008). Beliefs about learning, self-regulated strategies and text comprehension among Chinese children. British Journal of Educational Psychology, 78, 51-73.

Lasley, T. J. (1980). Preservice teacher beliefs about teaching. Journal of Teacher Education, $31(4), 38-41$.

Lau, F. K., \& Nie, Y. (2008). Interplay between personal goals and classroom goal structures in predicting student outcomes: A multilevel analysis of person-context interactions. Journal of Educational Psychology, 100, 15-29.

Lave, J., \& Wenger, E. (1991). Situated learning: Legitimate peripheral participation. New York: Cambridge University Press.

Leavy, A. M., McSorley, F. A., \& Bote, L. A. (2007). An examination of what metaphor construction reveals about the evolution of preservice teachers' beliefs about teaching and learning. Teaching and Teacher Education, 23, 1217-1235.

Lederman, N. G. (1992). Students' and teachers' conceptions of the nature of science: A review of the research. Journal of Research in Science Teaching, 29, 331-359.

Lehrer, K. (1990). Theory of knowledge. San Francisco, CA: Westview Books.

Lemos, M. S. (1999). Students' goal and self-regulation in the classroom. International Journal of Educational Research, 31, 471-485.

Leschinsky, A., \& Cortina, K. S. (2008). Zur sozialen Einbettung bildungspolitischer Trends in der Bundesrepublik. In K. S. Cortina, J. Baumert, A. Leschinsky, K. U. Mayer, \& L. Trommer (Hrsg.), Das Bildungswesen in der Bundesrepublik Deutschland (S. 21-51). Reinbeck: Rowohlt Taschenbuch Verlag.

Levin, B., \& He, Y. (2008). Investigating the content and sources of teacher candidates' personal practical theories (PPTS). Journal of Teacher Education, 59(1), 55-68.

Levitt, K. E. (2001). An analysis of elementary teachers' beliefs regarding the teaching and learning of science. Science Education, 86, 1-22. 
Lipowsky, F., Thußbas, C., Klieme, E., Reusser, K., \& Pauli, C. (2003). Professionelles Lehrerwissen, selbstbezogene Kognitionen und wahrgenommene Schulumwelt Ergebnisse einer kulturvergleichenden Studie deutscher und Schweizer Mathematiklehrkräfte. Unterrichtswissenschaft, 31(3), 206-237.

Lübeck, D. (2009). Lehransätze in der Hochschullehre. Dissertation zu Erlangung des akademischen Grades Doktor der Philosophie (Dr. phil.). Freie Universität Berlin.

Maggioni, L., \& Parkinson, M. M. (2008). The role of teacher epistemic cognition, epistemic beliefs, and calibration in instruction. Educational Psychology Review, 20, 445-461.

Magolda, M. B. B. (1996). Epistemological development in graduate and professional education. Review of Higher Education, 19(3), 283-304.

Mahlios, M., \& Maxson, M. (1998). Metaphors as structures for elementary and secondary preservice teachers' thinking. International Journal of Educational Research, 29, 227240.

Marland, P. (1995). Implicit theories of teaching. In L. W. Anderson (Ed.), International encyclopedia of teaching and teacher education (pp. 131-136). New York: Pergamon.

Marland, P. (1998). Teachers' practical theories: Implications for preservice teacher education. Asia-Pacific Journal of Teacher Education \& Development, 1(2), 15-23.

Martin, E., \& Balla, M. (1991). Conceptions of teaching and implications for learning. In B. Wright, Research and development in higher education, 13 (pp. 298-304). Campbelltown, NSW: Higher Education Research and Development Society of Australasia (HERDSA).

Martínez, M., Sauleda, N., \& Huber, G. (2001). Metaphors as blueprints of thinking about teaching and learning. Teaching and Teacher Education, 17, 965-977.

Marton, F., Dall'Alba, G., \& Beaty, E. (1993). Conceptions of learning. International Journal of Educational Research, 19, 277-300.

McComas, W. F., \& Olson, J. K. (1998). The nature of science in international science education standards documents. In W. F. McComas (Ed.), The nature of science in science education: Rationales and strategies (pp. 41-52). The Netherland: Kluwer Academic Publisher.

McLeod, D. B. (1992). Research on affect in mathematics: A reconceptualization. In D. A. Grouws (Ed.), Handbook of research on mathematics teaching and learning (pp. 575596). New York: MacMillan 
Meirink, J. A., Meijer, P. C., Verloop, N., \& Bergen, T. C. M. (2009). Understanding teacher learning in secondary education: The relations of teacher activities to changed beliefs about teaching and learning. Teaching and Teacher Education, 25, 89-100.

Meyer, J., \& Eley, M. (2003). A factor analysis of the approach to teaching inventory. Paper presented at the 10th EARLI, Padua (Italy). Retrieved from http://www.celts.monash.edu.au/assets/other/earli03-meyer-and-eley-paper.pdf

Millar, R. (1989). Bending the evidence: The relationship between theory and experiment in science education. In R. Millar (Ed.), Doing science: Images of science in science education (pp. 38-61). New York: the Falmer Press.

Morgan, A., \& Beaty, E. (1997). The world of the learner. In F. Marton, D. J. Hounsell, \& N. J. Entwistle (Eds.), The experience of learning (pp. 217-237). Edinburgh: Scottish Academic Press.

Morine-Dershimer, G., \& Kent, T. (1999). The complex nature and sources of teachers' pedagogical knowledge. In J. Gess-Newsome, \& N. G. Lederman (Eds.), Examining pedagogical content knowledge. The construct and its implications for science education (pp. 21-50). Dordrecht.

Muis, K. R., Bendixen, L. D., \& Haerle, F. (2006). Domain-generality and domain-specifity in personal epistemology research: Philosophical and empirical reflections in the development of a theoretical framework. Educational Psychology Review, 18, 3-54.

Munby, H. (1987). Metaphor and teacher's knowledge. Research in the Teaching of English, 21(4), 377-397.

Murray, K., \& MacDonald, R. (1997). The disjunction between lecturers' conceptions of teaching and their claimed educational practice. Higher Education, 33, 331-349.

Nespor, J. (1987). The role of beliefs in the practice of teaching. Journal of Curriculum Studies, 19, 317-328.

Nettle, E. B. (1996). The development and use of scales for measuring student teachers' beliefs about teaching (Unpublished doctoral thesis). The University of New South Wales, Sydney.

Nettle, E. B. (1998). Stability and change in the beliefs of student teachers during practice teaching. Teaching and Teacher Education, 14(2), 193-204.

Ng, W., Nicholas, H., \& Williams, A. (2010). School experience influences on pre-service teachers' evolving beliefs about effective teaching. Teaching and Teacher Education, 26, 278-289. 
Nisbett, R. E., \& Wilson, T. D. (1977). Telling More Than We Can Know: Verbal Reports on Mental Processes. Psychological Review, 84(3), 231-259.

Nisbett, R., \& Ross, L. (1980). Human inference: Strategies and shortcoming of social judgment. NJ: Prentice-Hall: Englewood Cliffs.

Norton, L., Richardson, J. T. E., Hartley, J., Newstead, S., \& Mayes, J. (2005). Teachers beliefs and intentions concerning teaching in higher education. Higher Education, 50, $537-571$.

OECD (2009). Creating Effective Teaching and Learning Environments: First results from TALIS 2008. Retrieved from http://www.oecd.org/dataoecd/17/51/43023606.pdf

Oevermann, U. (1996). Theoretische Skizze einer revidierten Theorie professionalisierten Handelns. In A. Combe, \& W. Helsper (Hrsg.), Pädagogische Professionalität (pp. 70-82). Frankfurt a.M.

Olafson, L., \& Schraw, G. (2006). Teachers' beliefs and practices within and across domains. International Journal of Educational Research, 45, 71-84.

Op't Eynde, P., De Corte, E., \& Verschaffel, L. (2006). Epistemic dimensions of students' mathematics-related beliefs systems. International Journal of Educational Research, $45,57-70$.

Oser, F. (1998). Ethos - Die Vermenschlichung des Erfolgs. Zur Psychologie der Berufsmoral von Lehrpersonen. Opladen.

Oser, F. (2009). Moral jenseits von organisierter Erlaubtbarkeit: Zur inneren und äußeren Effizienz eines professionellen Ethos. In O. Zlatkin-Troitschanskaia, K. Beck, D. Sembill, R. Nickolaus \& R. H. Mulder (Hrsg.), Lehrerprofessionalität - Bedingungen, Genese, Wirkungen und Messung (S. 389-400). Weinheim: Beltz.

Oser, F., \& Oelkers, J. (2001). Die Wirksamkeit der Lehrerbildungssysteme. Von der Allroundbildung zur Ausbildung professioneller Standards. Zürich: Verlag Rüegger.

Oxford, R., Tomlinson, S., Barcelos, A., Harrington, C., Lavine, R., Saleh, A. et al. (1998). Clashing metaphors about classroom teachers: toward a systematic typology for the language teaching field. System, 26, 3-50.

Pajares, M. F. (1992). Teachers' beliefs and educational research: Cleaning up a messy construct. Review of Educational Research, 3, 62(3), 307-332.

Palincsar, A. S., \& Brown, A. L. (1984). Reciprocal teaching of comprehension-fostering and comprehension-monitoring activities. Cognition \& Instruction, 1, 117-175.

Paulick, I., Watermann, R., \& Nückles, M. (2011). Zielorientierungen und schulisches Lernen am Grundschulübergang. Unterrichtswissenschaft, 39(4), 365-384. 
Perry, W. G. (1970). Forms of intellectual and ethical development in the college years. New York: Holt, Rinehart \& Winston.

Peterson, P. L., Fennema, E., Carpenter, T. P., \& Loef, M. (1989). Teachers' pedagogical content beliefs in mathematics. Cognition \& Instruction, 6(1), 1-40.

Phan, H. P. (2008). Predicting change in epistemological beliefs, reflective thinking und learning styles: A longitudinal study. British Journal of Educational Psychology, 78, 75-93.

Piaget, J. (1978). Das Weltbild des Kindes. München: dtv/Klett-Cotta.

Pillow, B. H. (1999). Epistemological development in adolescence and adulthood: A multidimensional framework. Genetic, Social, and General Psychology Monographs, $125,413-432$.

Pintrich, P. R. (1990). Implication of psychological research on student learning and college teaching for teacher education. In W. R. Houston (Ed.), Handbook of Research on Teacher Education (pp. 826-857). New York: Macmillan.

Pollock, J. L. (1986). Contemporary theories of knowledge. Savage, MD: Rowman \& Littlefield.

Postareff, L., \& Lindblom-Ylänne, S. (2008). Variation in teachers' description of teaching: Broadening the understanding of teaching in higher education. Learning and Instruction, 18, 109-120.

Potari, D., \& Georgiadou-Kabouridis, B. (2009). A primary teacher's mathematics teaching: The development of beliefs and practice in different "supportive" contexts. Journal of Mathematics Teacher Education, 12, 7-25.

Pratt, D. D. (1992). Conceptions of teaching. Adult Education Quarterly, 42(4), 203-220.

Pratt, D. D. (2002). Good Teaching: One Size Fits All? New Directions for Adult and Continuing Education, 93, 5-15.

Pratt, D. D. (2005). Five perspectives on teaching in adult and higher education. Reprinted edition with corrections. Malabar, Florida: Krieger Publishing

Pratt, D. D., Collins, J. B., \& Jarvis-Selinger, S. (2001). Development and use of the teaching perspectives inventory (TPI). Paper presented at the Annual Meeting of the American Educational Research Association (AERA). Retrieved from http://www.teachingperspectives.com/PDF/development1.pdf

Prenzel, M. (Ed). (2007). Studies on the Educational Quality of Schools. The final report on the DFG Priority Programme. Münster: Waxmann. 
Quian, G., \& Alvermann, D. (1995). Role of epistemological beliefs and learned helplessness in secondary school students' learning science concepts from text. Journal of Educational Psychology, 87(2), 282-292.

Quian, G., \& Alvermann, D. (2000). Relationship between epistemological beliefs and conceptual change learning. Reading Writing Quarterly, 16(1), 59-74.

Radtke, F.-O. (2004). Der Eigensinn pädagogischer Professionalität jenseits von Innovationshoffnungen und Effizienzerwartungen. Übergangene Einsichten aus der Wissensverwendungsforschung für die Organisation universitärer Lehrerbildungsforschung. In B. Koch-Priewe, F.-U. Kolbe, \& J. Wildt (Hrsg.), Grundlagenforschung und mikrodidaktische Reformansätze zur Lehrerbildung (S. 99149). Bad Heilbrunn.

Rathbone, C., \& Pierce, J. (1989). Accommodation of personal teaching style as a function of the student teaching experience. Paper presented at the Northeast Educational Research Organization, Middleown, NY.

Raymond, A. M. (1997). Inconsistency between a beginning elementary school teacher's mathematics beliefs and teaching practice. Journal for Research in Mathematics Education, 28, 550-576.

Resnick, L. B., \& Hall, M. W. (1998). Learning organizations for sustainable education reform. Daedalus, 127, 89-118.

Reusser, K. (2008). Empirisch fundierte Didaktik: Didaktisch fundierte Unterrichtsforschung. Zeitschrift für Erziehungswissenschaft (Sonderheft 9), 219-238.

Richardson, V. (1996). The role of attitudes and beliefs in learning to teach. In J. Sikula (Ed.), Handbook of research on teaching (pp. 376-391). New York: Macmillan.

Richardson, V. (2003). Preservice teachers' beliefs. In J. Raths \& A. McAninch (Ed.), Teacher belief and teacher education. Advantages in teacher education (pp. 1-22). Greenwich, CT: Information Age Publishers.

Rogers, C. R. (1969). Freedom to learn. New York: MacMillan.

Rogers, C. R., \& Freiberg, H. J. (1994). Freedom to learn (3rd ed.). New York: MacMillan.

Rogoff, B. (1990). Apprenticeship in Thinking: Cognitive Development in Social Context. Oxford, UK: Oxford University Press.

Rokeach, M. (1968). Beliefs, attitudes, and values. San Francisco: Jossey-Bass

Rost, D. H. (2007). Interpretation und Bewertung pädagogisch-psychologischer Studien. Eine Einführung. Weinheim: Beltz. 
Rukavina, I., \& Daneman, M. (1996). Integration and its effects in acquiring knowledge about competing from text. Journal of Educational Psychology, 88(2), 272-287.

Russel, T. (1988). From preservice teacher education to first year of teaching: A study of theory and practice. In J. Calderhead (Ed.), Teachers' Professional Learning (pp. 1334). London: Falmer Press.

Rust, F. O. (1994). The first year of teaching: It's not what they expected. Teaching and Teacher Education, 10(2), 205-217.

Ruthven, K. (2002). Linking researching with teaching: Toward synergy of scholarly and craft knowledge. In L. English (Ed.), Handbook of international research in mathematics education (pp. 581-598). NJ: Lawrence Erlbaum.

Ryan, A., \& Patrick, H. (2001). The classroom social environment and changes in adolescents' motivation and engagement during middle school. American Educational Research Journal, 38(2), 437-460.

Saban, A., Kocbeker, B. N., \& Saban, A. (2007). Prospective teachers' conceptions of teaching and learning revealed through metaphor analysis. Learning and Instruction, $17,123-139$.

Saban, A. (2010). Prospective teachers' metaphorical conceptualizations of learner. Teaching and Teacher Education, 26, 290-305.

Salhab, M. (2003). Interdisziplinäres Textverstehen am Beispiel ethnischer Schutzkonzepte in der Stammzellenforschung. (Unveröffentlichte Diplomarbeit). Albert-LudwigsUniversität, Freiburg.

Scardamalia, M., \& Bereiter, C. (1987). Knowledge telling and knowledge transforming in written composition. In S. Rosenberg (Ed.), Advances in applied psycholinguistics: Vol. 2. Reading, writing, and language learning (pp. 142-175). Cambridge: Cambridge University Press.

Schiefele, U., Moschner, B., \& Husstegge, R. (2002). Skalenhandbuch SMILE-Projekt. Unveröffentlichtes Manuskript, Universität Bielefeld, Universität Bielefeld.

Schommer, M. (1990). Effects of beliefs about the nature of knowledge on comprehension. Journal of Educational Psychology, 82(3), 498-504

Schmidt, A. (1991). Das Gymnasium im Aufwind. Entwicklung, Struktur, Probleme seiner Oberstufe. Aachen: Hahner Verlagsgesellschaft.

Schommer, M. (1992). Predictors of epistemological beliefs: Comparison adults with only a secondary education to adults with post-secondary education. Paper presented at the Midwestern Educational Research Association, Chicago, IL. 
Schommer, M. (1993). Epistemological development and academic performance among secondary students. Journal of Educational Psychology, 85(3), 406-411.

Schommer, M. (1994a). An emerging conceptualization of epistemological beliefs and their role in learning. In R. Garner, \& A. P. Alexander (Eds.), Beliefs about text and about text instruction (pp. 25-39). Hillsdale, NJ: Lawrence Erlbaum.

Schommer, M. (1994b). Synthesizing epistemological belief of research: Tentative understandings and provocative confusions. Educational Psychology Review, 6(4), $293-319$.

Schommer, M. (1998). The influence of age and education on epistemological beliefs. British Journal of Educational Psychology, 68, 551-562.

Schommer, M., Clavert, C., Ganglietti, G., \& Baja, A. (1997). The development of epistemological beliefs among secondary students: A longitudinal study. Journal of Educational Psychology, 89(1), 37-40.

Schommer, M., Crouse, A., \& Rhodes, N. (1992). Epistemological beliefs and mathematical text comprehension: Believing it is simple does not make it so. Journal of Educational Psychology, 84(4), 435-443.

Schoenfeld, A. H. (1983). Beyond the purely cognitive: Belief systems, social cognitions, and metacognitions as driving forces in intellectual performance. Cognitive Science, 7, $329-363$.

Schoenfeld, A. H. (1985). Mathematical problem solving. CA: San Diego Academic Press.

Schraw, G., Bendixen, L. D., \& Dunkle, M. E. (2002). Development and validation of the epsitemic belief inventory (EBI). In B. K. Hofer, \& P. R. Pintrich (Eds.), Personal epistemology: The psychology of beliefs about knowledge and knowing (pp. 261-275). Mahwah, NJ: Erlbaum.

Schraw, G., Dunkle, M. E., \& Bendixen, L. D. (1995). Cognitive Processes in Well-Defined and Ill-Defined Problem Solving. Applied Cognitive Psychology, 9, 523-538.

Schraw, G., \& Olafson, L. (2002). Teachers' epistemological worldviews and educational practice. Issues in Education, 8, 99-148.

Schraw, G., \& Olafson, L. (2008). Assessing Teachers' Epistemological and Ontological Worldviews. In M. S. Khine (Ed.), Knowing, Knowledge and Beliefs: Epistemological Studies 25 across Diverse Cultures. Springer.

Seidel, T., Schwindt, K., Rimmele, R., \& Prenzel, M. (2008). Konstruktivistische Überzeugungen von Lehrpersonen: Was bedeuten sie für den Unterricht? Zeitschrift für Erziehungswissenschaft (Sonderheft 9, Perspektiven der Didaktik). (S. 259-276). 
Sfard, A. (1998). On two metaphors for learning and the dangers of choosing just one. Educational Researcher, 27(2), 4-13.

Shulman, L. S. (1986). Those who understand: Knowledge growth in teaching. Educational Researcher, 15(2), 4-14.

Shulman, L. S. (1987). Knowledge and teaching: Foundation of the new reform. Harvard Educational Review, 57(1), 1-22.

Sigel, I. E. (1985). A conceptual analysis of beliefs. In I. E. Sigel (Ed.), Parental beliefs systems: The psychological consequences for children (pp. 345-371). Hillsdale, NJ: Erlbaum.

Sinatra, G. M. (2001). Knowledge, beliefs, and learning. Educational Psychology Review, 13(4), 321-323.

Skinner, B. F. (1973). Wissenschaft und menschliches Verhalten. Science and Human Behavior. München: Kindler.

Skinner, B. F. (1978). Was ist Behaviorismus? Reinbeck: Rowohlt Taschenbuch Verlag.

Skott, J. (2002). Belief research and the actual and virtual communities of a novice teacher's practice. In A. D. Cockburn \& E. Nardi (Eds.), Proceedings of the 26th Annual Conference of the International Group for the Psychology of Mathematics Education (Vol. 4). Norwich.

Speer, N. M. (2005). Issues of methods and theory in the study of mathematics teachers' professed and attributed beliefs. Educational Studies in Mathematics, 58, 361-391.

Stahl, E., \& Bromme, R. (2007). The CAEB: An instrument for measuring connotative aspects of epistemological beliefs. Learning and Instruction, 17, 773-785.

Stathopoulou, C., \& Vosniadou, S. (2007). Exploring the relationship between physics-related epistemological beliefs and physics understanding. Contemporary Educational Psychology, 32, 255-281.

Staub, F. C., \& Stern, E. (2002). The nature of teachers' pedagogical content beliefs matters for students' achievement gains: Quasi-experimental evidence from elementary mathematics. Journal of Educational Psychology, 94(2), 344-355.

Sternberg, R. J., \& Horvath, J. H. (Eds.). (1999). Tacit knowledge in professional practice. Mahwah, NJ: Erlbaum.

Stipek, D. J. (1996). Motivation and instruction. In D. Berliner, \& R. Calfee (Eds.), Handbook of educational psychology (pp. 85-113). New York: Macmillan.

Stipek, D. J. (1998). Motivation to learn: From theory to practice (3rd ed.). Needham Heights, MA: Allyn and Bacon. 
Stipek, D. J., Givvin, K. B., Salmon, J. M., \& MacGyvers, V. L. (2001). Teachers' beliefs and practices related to mathematics instruction. Teaching and Teacher Education, 17(2), 213-226.

Stipek, D. J. (2004). Teaching practices in kindergarten and first grade: Different strokes for different folks. Early Childhood Research Quarterly, 19, 548-568.

Stofflett, R. T., \& Stoddart, T. (1991). The effect of the content instruction on elementary teacher candidates use of conceptual change strategies. Paper presented at the annual meeting of the American Educational Research Association, Chicago.

Terhart, E. (Hrsg.) (2000). Perspektiven der Lehrerausbildung. Abschlussbericht der von der Kultusministerkonferenz eingesetzten Kommission. Weinheim, Basel: Beltz.

Thompson, A. (1984). The relationship of teachers' conceptions of mathematics and mathematics teaching to instructional practice. Educational Studies in Mathematics, $15,105-127$.

Thompson, A. (1985). Experience, problem solving, and learning mathematics: Consideration in developing mathematics curricula. In E. Silver (Ed.), Teaching and learning mathematical problem solving: Multiple research perspectives (pp. 189-236). Hillsdale, NJ: Erlbaum.

Thompson, A. (1992). Teachers' beliefs and conceptions: A synthesis of the research. In D.

A. Grouws (Ed.), Handbook of research on mathematics teaching and learning (pp. 127-146). New York: MacMillan.

Tillema, H. H. (1998). Stability and change in student teachers' beliefs about teaching. Teachers and Teaching: Theory and Practice, 4, 217-285.

Trautwein, U., \& Lüdtke, O. (2004). Wissenschaftspropädeutik in der gymnasialen Oberstufe. In O. Köller, R. Watermann, U. Trautwein \& O. Lüdtke (Hrsg.), Wege zur Hochschulreife in Baden-Württemberg: TOSCA - eine Untersuchung an allgemein bildenden und beruflichen Gymnasien (S. 351-366). Opladen: Leske + Budrich.

Trautwein, U., \& Lüdtke, O. (2007). Epistemological beliefs, school achievement, and college major: A large-scale longitudinal study on the impact of certainty beliefs. Contemporary Educational Psychology, 32(3), 348-366.

Treagust, D. F., Duit, R., \& Fraser, B. J. (1996). Overview: Research on students' preinstructional conceptions - The driving force for improving teaching and learning in science and mathematics. In D. F. Treagust, R. Duit, \& B. J. Fraser (Eds.), Improving Teaching and Learning in Science and Mathematics (pp. 1-16). New York. 
Trigwell, K., Prosser, M., \& Taylor, P. (1994). Qualitative differences in approaches to teaching first year university science. Higher Education, 27, 75-84.

Trigwell, K., \& Prosser, M. (2004). Development and use of the approaches to teaching inventory. Educational Psychology Review, 16(4), 409-424.

Trigwell, K., Prosser, M., \& Ginns, P. (2005). Phenomenographic pedagogy and a revised Approaches to Teaching Inventory. Higher Education Research and Development, 24(4), 349-360.

Tsai, C. C. (2006). Reinterpreting and reconstructing science: Teacher's view changes toward the nature of science by courses of science education. Teaching and Teacher Education, 22(3), 363-375.

Van Driel, J. H., Bulte, A. M. W., \& Verloop, N. (2007). The relations between teachers' general beliefs about teaching and learning and their domain specific curricular beliefs. Learning and Instruction, 17, 156-171.

Veenman, S. (1984). Perceived problems of beginning teachers. Review of Educational Research, 54, 143-178.

Verloop, N., Van Driel, J. H., \& Meijer, P. C. (2001). Chapter 1: Teacher knowledge and the knowledge base of teaching. International Journal of Educational Research, 35, 441461.

Voss, T., Kleickmann, T., Kunter, M. \& Hachfeld, A. (2011). Überzeugungen von Mathematiklehrkräften. In M. Kunter, J. Baumert, W. Blum, U. Klusmann, S. Krauss, \& M. Neubrand (Hrsg.): Professionelle Kompetenz von Lehrkräften. Ergebnisse des Forschungsprogramms COACTIV. (S. 235-257) Münster: Waxmann.

Waldis, M., Grob, U., Pauli, C., \& Reusser, K. (2010). Der schweizerische Mathematikunterricht aus der Sicht von Schülerinnen und Schülern und in der Perspektive hochinferenter Beobachterurteile. In K. Reusser, C. Pauli \& M. Waldis (Hrsg.): Unterrichtsgestaltung und Unterrichtqualität: Ergebnisse einer internationalen und schweizerischen Videostudie zum Mathematikunterricht. (S. 209251) Münster: Waxmann.

Weinstein, C. (1989). Teacher education students' perceptions of teaching. Journal of Teacher Education, 40(2), 53-60.

Weinstein, C. S. (1990). Prospective elementary teachers' beliefs about teaching: Implication for teacher education. Teaching and Teacher Education, 6(3), 279-290

Wentzel, K. R. (1997). Student motivation in the middle school: The role of perceived pedagogical caring. Journal of Educational Psychology, 89, 411-419. 
Wideen, M., Mayer-Smith, J., \& Moon, B. (1998). A critical analysis of the research on learning to teach: Making the case for an ecological perspective on inquiry. Review of Educational Research, 68(2), 130-178.

Wilcox-Herzog, A. (2002). Is there a link between teachers' beliefs and behaviors? Early Education and Development, 13, 79-106.

Winteler, A. (2001). Lehrende an Hochschulen. In: A. Krapp \& B. Weidenmann (Hrsg.), Pädagogische Psychologie (S. 332-345). Weinheim: Beltz PVU.

White, R. T., \& Gunstone, R. F. (1992). Probing understanding. London: Flamer Press.

White, B. C. (2000). Pre-service teachers' epistemology viewed through perspectives on problematic classroom situations. Journal of Education for Teaching, 26, 279-305.

Wolters, C., Yu, S., \& Pintrich, P. (1996). The relation between goal orientation and students' motivational beliefs and self-regulated learning. Learning and Individual Differences, $8,211-238$.

Wood, T., Cobb, P., \& Yackel, E. (1991). Change in teaching mathematics: A case study. American Educational Research Journal, 28(3), 587-616.

Woolfolk-Hoy, A., Davis, H., \& Pape, S. J. (2006). Teacher knowledge and beliefs. In P. A. Alexander \& P. H. Winne (Eds.), Handbook of educational psychology (2nd ed., pp. 715-737). Mahwah, NJ: Erlbaum.

Zeichner, K., \& Tabachnick, B. R. (1981). Are the effects of university teacher education washed out by the school experience? Journal of Teacher Education, 3(3), 7-11.

Zeichner, K., \& Liston, D. P. (1987). Teaching student teachers to reflect. Harvard Educational Review, 57, 23-47.

Zhang, L. F., \& Watkins, D. (2001). Cognitive development and student approaches to learning: An investigation of Perry's theory with Chinese and U.S. university students. Higher Education, 41, 239-261. 


\section{Anhang A: Überblick über die verschiedenen Überzeugungen in ihrer Relation zueinander}

\begin{tabular}{|c|c|c|c|c|c|c|c|c|}
\hline \multirow[b]{2}{*}{$\begin{array}{l}\text { Quelle } \\
\text { Alger (2009) } \\
\text { Conceptual } \\
\text { metaphors of } \\
\text { teaching and } \\
\text { learning }\end{array}$} & \multicolumn{8}{|l|}{ Überzeugung } \\
\hline & $\begin{array}{l}\text { Teaching as } \\
\text { molding } \\
\text { (teacher-centered) } \\
\text { I am seated at a } \\
\text { potter's wheel with } \\
\text { a lump of clay. I } \\
\text { carefully mold the } \\
\text { clay into a well } \\
\text { shaped and } \\
\text { beautiful vase. } \\
\text { Sometimes it takes } \\
\text { pushing and } \\
\text { prodding to get the } \\
\text { vase to develop. }\end{array}$ & \multicolumn{3}{|c|}{$\begin{array}{l}\text { Teaching as } \\
\text { transmitting } \\
\text { (teacher-centered) } \\
\text { I have a large sum } \\
\text { of money, which I } \\
\text { deposit into a series } \\
\text { of accounts. The } \\
\text { goal is to deposit as } \\
\text { much money as I } \\
\text { can into each } \\
\text { account so that each } \\
\text { account has a high } \\
\text { balance. }\end{array}$} & $\begin{array}{l}\text { Teaching as } \\
\text { nurturing } \\
\text { (teacher-centered) } \\
\text { It is a sunny day. I } \\
\text { see myself holding a } \\
\text { watering can and } \\
\text { carefully attending } \\
\text { to my seedlings. I } \\
\text { make sure that the } \\
\text { soil, water, and } \\
\text { climate are rich and } \\
\text { right for each } \\
\text { seedling so that each } \\
\text { will develop and } \\
\text { blossom }\end{array}$ & \multicolumn{2}{|c|}{$\begin{array}{l}\text { Teaching as providing tools } \\
\text { (student-centered) } \\
\text { I wear a large tool belt. As each worker constructs his } \\
\text { or her house, I provide the builder with the tools he or } \\
\text { she will need to be successful in completing the } \\
\text { project. }\end{array}$} & $\begin{array}{l}\text { Teaching as } \\
\text { engaging in } \\
\text { community } \\
\text { (student-centered) } \\
\text { I am part of a } \\
\text { community that is } \\
\text { building a house. We } \\
\text { collectively decided } \\
\text { that we need a house } \\
\text { and then we design } \\
\text { and build it together. }\end{array}$ \\
\hline $\begin{array}{l}\text { Boulton- } \\
\text { Lewis, et al } \\
\text { (2001) } \\
\text { Conceptions } \\
\text { of teaching }\end{array}$ & \multicolumn{2}{|c|}{$\begin{array}{l}\text { Transmission of content/skills } \\
\text { (teacher-and-content-focus) } \\
\text { teaching as imparting information or } \\
\text { skills, } \\
\text { teacher \& content is in focus vs. } \\
\text { student is in the background }\end{array}$} & \multicolumn{2}{|c|}{$\begin{array}{l}\text { Development of skills/ } \\
\text { understanding } \\
\text { (teacher-to-student-focus) } \\
\text { teacher directs the learning } \\
\text { process, } \\
\text { students as participants, focus } \\
\text { on students' achieving the } \\
\text { teacher's level of skill }\end{array}$} & & $\begin{array}{l}\text { Facilitating of } \\
\text { understanding } \\
\text { (teacher-student } \\
\text { interaction focus) } \\
\text { both teacher \& students } \\
\text { are involved in the } \\
\text { process of working } \\
\text { together to construct } \\
\text { personal meaning }\end{array}$ & $\begin{array}{l}\text { Transformation of student } \\
\text { (student focus) } \\
\text { teacher organizes the } \\
\text { situation to provide the } \\
\text { stimulus \& then apparently } \\
\text { fading into the background } \\
\text { for students to take action to } \\
\text { develop themselves, to } \\
\text { extend cognitively/ } \\
\text { behaviorally/affectively }\end{array}$ & \\
\hline $\begin{array}{l}\text { Boulton- } \\
\text { Lewis et al. } \\
\text { (2001) } \\
\text { Conceptions } \\
\text { of learning }\end{array}$ & \multicolumn{2}{|c|}{$\begin{array}{l}\text { Acquisition and reproduction of } \\
\text { content/skills } \\
\text { (content focus) } \\
\text { Learning is for student to take in ( } \& \\
\text { use) information/skills by } \\
\text { listening/repetition }\end{array}$} & \multicolumn{2}{|c|}{$\begin{array}{l}\text { Development \& application } \\
\text { of skills/understanding } \\
\text { (competence focus) } \\
\text { Learning is for student to be } \\
\text { equipped/able//understand/appl } \\
\text { y by doing practical } \\
\text { tasks/relating it to own } \\
\text { experience/putting it into } \\
\text { practices/solving problems }\end{array}$} & & $\begin{array}{l}\text { Development of } \\
\text { understanding } \\
\text { (meaning focus) } \\
\text { Learning is when } \\
\text { students } \\
\text { develop/discover/constru } \\
\text { ct mental } \\
\text { frameworks/concepts/un } \\
\text { derstanding by } \\
\text { discussion/ group } \\
\text { work/thinking/ } \\
\text { metacognition }\end{array}$ & $\begin{array}{l}\text { Transformation of learners } \\
\text { (growth focus) } \\
\text { Learning is when students } \\
\text { grow/change/develop } \\
\text { cognitively/ behaviorally/ } \\
\text { affectively by } \\
\text { exploring/discovering/risking/ } \\
\text { questioning/resoning/critizing } \\
\text { /succeeding }\end{array}$ & \\
\hline
\end{tabular}




\begin{tabular}{|c|c|c|c|c|c|c|c|c|}
\hline Quelle & \multicolumn{8}{|c|}{ Überzeugungen } \\
\hline $\begin{array}{l}\text { Dunkin \& } \\
\text { Precians } \\
(1992) \\
\text { Teachers' } \\
\text { concepts of } \\
\text { teaching }\end{array}$ & \multicolumn{2}{|c|}{$\begin{array}{l}\text { Structuring learning } \\
\text { Teachers caters for contents, which are } \\
\text { important to be learned for examination } \\
\text { purpose, helps the students to learn, so that } \\
\text { they get good notes. He encounters the } \\
\text { feelings by the student, that the contents are } \\
\text { new, so it's necessary to relate the new } \\
\text { material to the previous experience. }\end{array}$} & $\begin{array}{l}\text { Motivating learning } \\
\text { The most important } \\
\text { thing is to get the } \\
\text { students motivated } \\
\text { and make them } \\
\text { enthusiastic about } \\
\text { the subject. First of } \\
\text { all is got to pass on } \\
\text { owns love of the } \\
\text { subject. }\end{array}$ & \multicolumn{4}{|c|}{$\begin{array}{l}\text { Encouraging activity and self-directed learning } \\
\text { involve the students in preparation for courses; through conversation with } \\
\text { students teacher relates to particular situation rather than give standards } \\
\text { answers, } \\
\text { students want to learn, the teacher minimize the fear of making mistakes, } \\
\text { because real scientists not always know the right answer. }\end{array}$} & $\begin{array}{l}\text { Establishing interpersonal } \\
\text { relationship } \\
\text { Relation to student in a } \\
\text { collaboration manner like real } \\
\text { scientists discussing with } \\
\text { somebody, who knows more } \\
\text { than one self and working } \\
\text { together. } \\
\text { The teachers as a person \& } \\
\text { not a fact-machine. } \\
\text { The teacher should arrange } \\
\text { some kind of personal relation } \\
\text { to students, if they need to } \\
\text { speak on an individual basic. }\end{array}$ \\
\hline $\begin{array}{l}\text { Kember } \\
\text { (1997) } \\
\text { Conceptions } \\
\text { of teaching }\end{array}$ & $\begin{array}{l}\text { Imparting } \\
\text { information } \\
\text { (content-oriented/ } \\
\text { teacher-centered) } \\
\text { Teaching as } \\
\text { presenting } \\
\text { information, as } \\
\text { lecturing \& } \\
\text { focusing upon the } \\
\text { note which are } \\
\text { prepared by the } \\
\text { teacher; } \\
\text { student as a passive } \\
\text { recipient of a body } \\
\text { of contents }\end{array}$ & $\begin{array}{l}\text { Transmitting } \\
\text { structured } \\
\text { knowledge } \\
\text { (content-oriented/ } \\
\text { teacher-centered) } \\
\text { Teaching as } \\
\text { transmitting of } \\
\text { structured \& arranged } \\
\text { presented knowledge } \\
\text { so that students have } \\
\text { more chance of } \\
\text { receiving the } \\
\text { information; } \\
\text { Student still } \\
\text { as a passive recipient }\end{array}$ & & $\begin{array}{l}\text { Student-teacher- } \\
\text { interaction/ } \\
\text { apprenticeship } \\
\text { The transitional } \\
\text { conception between } \\
\text { teacher- \& student- } \\
\text { focused conceptions; } \\
\text { the importance of an } \\
\text { interaction between } \\
\text { teacher \& student }\end{array}$ & $\begin{array}{l}\text { Facilitation } \\
\text { understanding } \\
\text { (Student- } \\
\text { centered/learning- } \\
\text { oriented) } \\
\text { focus on students } \\
\text { teaching as helping } \\
\text { students to learn; } \\
\quad \text { Importance } \\
\text { of students' learning } \\
\text { outcomes rather than } \\
\text { defining contents; } \\
\text { The students as } \\
\text { individuals rather than } \\
\text { as an audience to be } \\
\text { lectured }\end{array}$ & $\begin{array}{l}\text { Cor } \\
\text { intel } \\
\text { deve } \\
\text { (Stu } \\
\text { cent } \\
\text { orier } \\
\text { chan } \\
\text { conc } \\
\text { argu } \\
\text { appl } \\
\text { conf } \\
\text { diffe } \\
\text { what } \\
\text { what } \\
\text { happ } \\
\text { holis } \\
\text { proc } \\
\text { estal } \\
\text { relat } \\
\text { teacl }\end{array}$ & $\begin{array}{l}\text { eptual change/ } \\
\text { ctual } \\
\text { pment } \\
\text { nt- } \\
\text { ed/learning- } \\
\text { d) } \\
\text { ing students' } \\
\text { ptions by } \\
\text { g about things, } \\
\text { ng ideas, being } \\
\text { nted by } \\
\text { nces between } \\
\text { ne thinks \& } \\
\text { ctually } \\
\text { ns; } \\
\text { c development } \\
\text { s resulting from } \\
\text { ishing } \\
\text { nship between } \\
\text { r \& students }\end{array}$ & \\
\hline $\begin{array}{l}\text { Martínez et al. } \\
\text { (2001) } \\
\text { Metaphorical } \\
\text { conceptions } \\
\text { of learning } \\
\text { and teaching }\end{array}$ & \multicolumn{2}{|c|}{$\begin{array}{l}\text { Behaviorist/ empiricist view } \\
\text { teacher as a transmitter of knowledge, } \\
\text { knowledge as externally determined product, } \\
\text { student as recipient }\end{array}$} & \multicolumn{4}{|c|}{$\begin{array}{l}\text { Cognitive view } \\
\text { learning as a schema construction, } \\
\text { student as actively constructor of not fixed knowledge, } \\
\text { which is individually and actively constructing by transforming } \\
\text { old schemata into new ones or by inductively developing new } \\
\text { schemata from the series of varying g experiences } \\
\text { teacher as a facilitator }\end{array}$} & \multicolumn{2}{|c|}{$\begin{array}{l}\text { Situative/ sociohistoric view } \\
\text { learning as a product of authentic participation } \\
\text { in the activities of a community of practitioners, } \\
\text { learning by dialogic exchanges and activity and } \\
\text { collaboration, } \\
\text { knowledge as distributed among individuals in a } \\
\text { social community }\end{array}$} \\
\hline
\end{tabular}




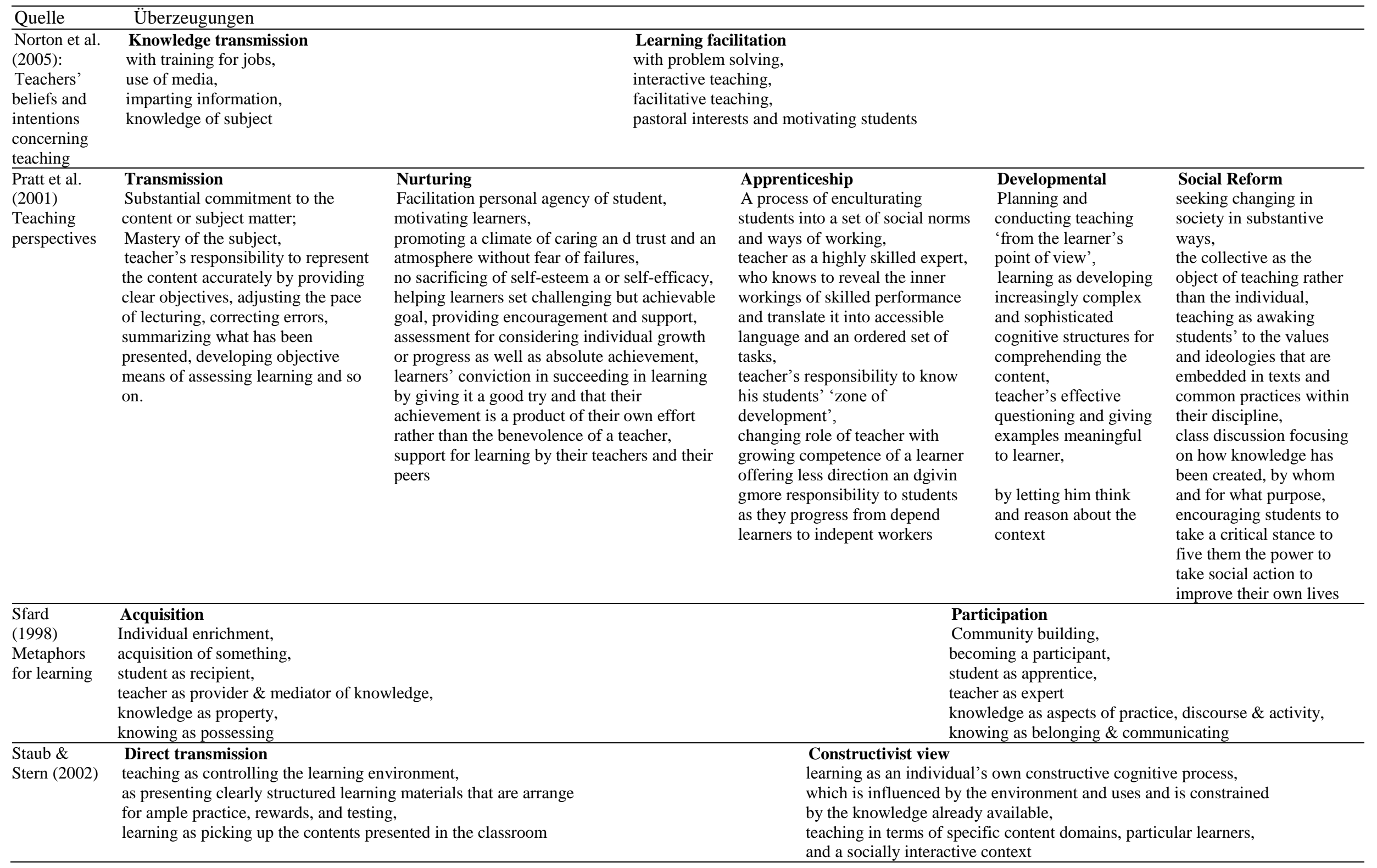




\begin{tabular}{|c|c|c|c|c|c|}
\hline Quelle & Überzeugungen & & & & \\
\hline $\begin{array}{l}\text { Trigwell et } \\
\text { al. (1994) } \\
\text { Approaches } \\
\text { to teaching }\end{array}$ & $\begin{array}{l}\text { A teacher-focused strategy } \\
\text { with the intention of } \\
\text { transmitting information to } \\
\text { students } \\
\text { teaching as transmitting facts } \\
\text { and transmitting demonstrated } \\
\text { skills, but not the relationship } \\
\text { between the facts or the skills, } \\
\text { prior knowledge of students as } \\
\text { not considered, } \\
\text { learning without being active } \\
\text { in the teaching-learning } \\
\text { process }\end{array}$ & $\begin{array}{l}\text { A teacher-focused strategy with } \\
\text { the intention of acquiring } \\
\text { concepts by students } \\
\text { teaching as helping students' } \\
\text { concepts of the discipline and the } \\
\text { relationship between them, } \\
\text { gaining of these concepts by the } \\
\text { students by being told or shown } \\
\text { the concepts and their } \\
\text { relationships, } \\
\text { learning as recall of facts and } \\
\text { solving problems as well as the } \\
\text { ability to relate concepts and } \\
\text { solve transfer problems }\end{array}$ & $\begin{array}{l}\text { A teacher/student interaction } \\
\text { strategy with the intention of } \\
\text { students acquirement of the } \\
\text { concepts } \\
\text { teaching as an interaction between } \\
\text { the students and the teacher with } \\
\text { the aim to help students acquire } \\
\text { the discipline based concepts and } \\
\text { the relationships between them, } \\
\text { gaining of the disciplinary } \\
\text { knowledge through active } \\
\text { engagement in the teaching- } \\
\text { learning process }\end{array}$ & $\begin{array}{l}\text { A student-focused } \\
\text { strategy with the intention } \\
\text { of students' development } \\
\text { of their conceptions } \\
\text { focus on students and their } \\
\text { learning, } \\
\text { teaching as helping students } \\
\text { to further develop the world } \\
\text { view or conceptions they } \\
\text { already hold, } \\
\text { student as a constructor of } \\
\text { knowledge in order to } \\
\text { develop the conceptions }\end{array}$ & $\begin{array}{l}\text { A student-focused strategy } \\
\text { with the intention of } \\
\text { students' changing of their } \\
\text { conceptions } \\
\text { teaching as helping students } \\
\text { cha change their world views } \\
\text { or conceptions of the } \\
\text { phenomena, } \\
\text { learning as constructing own } \\
\text { knowledge, } \\
\text { learning as re-construction of } \\
\text { the own knowledge to } \\
\text { produce a new world view or } \\
\text { conceptions }\end{array}$ \\
\hline
\end{tabular}


Anhang B: Mittelwerte (M) und Standardabweichungen (SD) der Items der 14-Faktoren-Lösung aus der Studie 2

Unterrichten bedeutet für mich...

$M \quad S D$

... dass die Schülerinnen und Schüler einander fragen und gegenseitig diese Fragen beantworten.

$2.78 \quad .61$

... dass sich die Schülerinnen und Schüler über die Lerninhalte austauschen.

... die Schülerinnen und Schüler gegenseitig Sachverhalte

erklären und erläutern zu lassen.

... dass die Schülerinnen und Schüler sich gegenseitig beim

Lernen unterstützen.

... das Wir-Gefühl in der Klasse zu fördern.

... das gemeinschaftlich geteilte Wissen in der Gruppe zu entwickeln.

...gemeinsames Wissen in einer Lerngemeinschaft aufzubauen.

... dass die Schülerinnen und Schüler voneinander lernen.

... in Lerngemeinschaft das Wissen zu teilen.

... das gemeinschaftliche Lernen unter den Schülerinnen und

Schülern zu fördern.

...zum Aufbau einer Lerngemeinschaft beizutragen.

... das Gemeinschaftsgefühl in der Klasse zu fördern.

... dass die Schülerinnen und Schüler das, was nicht verstanden wurde, einander erklären und diese Erklärungen auch einfordern.

... dass die Schülerinnen und Schüler in einen Diskurs über die Lerninhalte treten.

... zu den Schülerinnen und Schülern eine Beziehung aufzubauen. so dass die Lehrperson ihre Schülerinnen und Schüler kennt. 
... die Beziehungen zu den Schülerinnen und Schülern zu stärken.

um die Schülerpersönlichkeiten gut zu kennen und diese als

Personen wahrnehmen zu können.

... die Lebenswelt der Schülerinnen und Schüler mit ihren Sorgen

und Freuden kennen zu lernen. um die Lernprozesse besser

verstehen zu können.

... die Schülerinnen und Schüler besser zu kennen. damit diese

Kenntnisse der Lehrperson helfen. das Lernen der Schülerinnen

und Schüler in einem realen Kontext zu verstehen.

... den Schülerinnen und Schülern Stoffe und Themen zur

Auswahl zu geben.

... die Schülerinnen und Schüler selbst darüber bestimmen zu

lassen. mit welchen Themen sie sich beschäftigen möchten.

... zu den Schülerinnen und Schülern eine Beziehung aufzubauen. damit sie die Lehrperson als einen Menschen auffassen und von ihr als Personen aufgefasst werden.

... bei der Stoffauswahl auf individuelle Wünsche der

Schülerinnen und Schülern einzugehen.

... die Wünsche der Schülerinnen und Schüler soweit wie möglich zu erfüllen.

... die Schülerinnen und Schüler besser kennen zu lernen. um ihre

Lernergebnisse in den Kontext ihrer Lebenssituation einordnen und dadurch besser verstehen zu können.

... sich als Lehrkraft auch um die Probleme der Schülerinnen und Schüler zu kümmern.

... bei der Themenwahl die Interesse der Schülerinnen und

Schüler einzubeziehen.

... mit den Schülerinnen und Schülern zu reden. wenn ihnen etwas nicht gefällt.

... Lerninhalte zu präsentieren. so dass die von den Schülerinnen und Schülern gut aufgenommen werden können.

...Lerninhalte für die Schülerinnen und Schülern gut nachvollziehbar zu referieren.

... Lerninhalte sorgfältig zu erläutern. so dass die Schülerinnen und Schüler sich diese gut merken können.

... Lerninhalte zu präsentieren. so dass sie für die Schülerinnen und Schüler gut nachvollziehbar sind. 
... den Schülerinnen und Schülern zu demonstrieren. wie

Verfahren ausgeführt werden.

$2.95 \quad .66$

... Vorgehensweisen zu zeigen. damit sich die Schülerinnen und Schüler daran orientieren können.

$3.16 \quad .57$

... das Gelerntes regelmäßig zu überprüfen. Damit das Wissen der

Schülerinnen und Schüle gefestigt werden kann.

$3.29 \quad .63$

... Lerninhalte regelmäßig zu überprüfen. um sicher zu gehen. dass die Schülerinnen und Schüler sich diese gut eingeprägt haben.

... Lerninhalte sorgfältig und genau zu erklären.

... regelmäßige Übungsaufgaben zu vergeben. die den

Schülerinnen und Schülern helfen. das Gelernte zu festigen.

...Arbeitsschritte vorzuführen. damit die Schülerinnen und

Schüler diese gut nachahmen können.

... Lerninhalte regelmäßig zu wiederholen. damit die

Schülerinnen und Schüler sich diese gut einprägen können.

... die Schülerinnen und Schüler mit ihren eigenen Ergebnissen auch einmal bewusst in Irre laufen zu lassen. bis sie es selbst gemerkt haben.

... auch Fehler der Schülerinnen und Schüler zu akzeptieren und sie selbst weitermachen zu lassen.

... Schülerinnen und Schüler zu ermutigen. ihre eigenen Lösungswege zu suchen. auch wenn sie ineffizient sind.

... auch Fehler der Schülerinnen und Schüler zu akzeptieren und sie weiter machen zu lassen. bis sie selbst gemerkt haben. dass

etwas nicht stimmt.

... es hinzunehmen. dass zum Lernen auch Fehler gehören. denn dadurch lernen die Schülerinnen und Schüler überhaupt erst. 
Anhang C: Faktorladungsmatrix, gebildete Skalen (Partizipation - P;

Schülerorientierung - SO; Transmission - T; Konstruktion-K), Anzahl der

Items (n) und Cronbachs Alpha ( $\alpha$ ) aus der Studie 3

Unterrichten bedeutet für mich...

$\begin{array}{llll}\text { F } 1 & \text { F } 2 & \text { F } 3 & \text { F } 4\end{array}$

... dazu beizutragen. dass sich die Schulklasse zu einer

$\begin{array}{llll}.86 & .28 & -.01 & .03\end{array}$

Lerngemeinschaft entwickelt.

... das gemeinschaftliche Lernen unter den Schülern zu fördern.

$\begin{array}{llll}.81 & .14 & .14 & -.03\end{array}$

... zum Aufbau einer Lerngemeinschaft beizutragen.

.79

$.20 \quad .01$

.028

... dass die Schüler gemeinsam lernen.

.69

$.09 \quad .12$

... dafür zu sorgen, dass die Schüler sich als Mitglieder .65

$.22-.10$

einer Lerngemeinschaft erfahren können.

... darauf $\mathrm{zu}$ achten, dass jeder Einzelne Teil einer Lerngemeinschaft werden kann.

.65

.25

... das Gemeinschaftsgefühl der Klasse zu fördern.

.63

.03

... gegenseitigen Respekt und soziale Zugehörigkeit unter den Schülerinnen zu unterstützen.

$\begin{array}{llll}\mathbf{5 6} & .07 & .33 & -.02\end{array}$

... eine angstfreie Lernatmosphäre zu schaffen, die zum Erproben der eigenen Lernfähigkeiten motiviert.

.53

$.19 \quad .32$

... die soziale Wertschätzung in der Gruppe der .50

.25

Lernenden zu unterstützen.

... herausfordernde Lernsituationen zu schaffen, die die $\begin{array}{llll}.35 & .09 & .29 & .33\end{array}$ Schüler meistern können und die ihr Selbstvertrauen stärken.

... eine Lernumgebung zu schaffen, in der die Schüler ihre eigenen Lernwege verfolgen können.

$\begin{array}{llll}.17 & .66 & .17 & .10\end{array}$


... eine Lernumgebung zu schaffen, die genügend Raum

.26

$\begin{array}{lll}.64 & .18 & -.00\end{array}$

für die eigenen Vorstellungen der Schüler lässt.

... die Schüler mit ihren eigenen Vermutungen auch

.20

$\mathbf{6 4}-.06$

.02

eigene Lernwege gehen zu lassen.

... den Schülern Stoffe und Themen zur Auswahl zu

.58

$-.07$ geben.

... die Schüler mit ihren eigenen alltäglichen

Erfahrungen Neues entdecken zu lassen.

... auch Fehler der Schüler zu akzeptieren und sie

weitermachen $\mathrm{zu}$ lassen, bis sie selbst gemerkt haben, dass etwas nicht stimmt.

... die Schüler mit ihren eigenen Vermutungen auch

einmal in die Irre gehen zu lassen, bis sie es selbst gemerkt haben.

... bei der Stoffauswahl auch auf individuelle Wünsche der Schüler einzugehen.

... bei der Themenauswahl die Interessen der Schüler einzubeziehen.

... die Schüler selbst darüber bestimmen zu lassen, mit erfüllen. 
... den Schülern zu helfen wie ein Freund.

... selbst ein Teil der Lerngemeinschaft zu sein.

... dass ich zusammen mit meinen Schülern ein gemeinsames Ziel verfolge.

... für Lernsituationen $\mathrm{zu}$ sorgen, in denen sich die Schüler kompetent erleben.

... obwohl Experte, ein Mitglied der Lerngemeinschaft mit gleichen Orientierungen zu sein.

... obwohl Experte, ein Mitglied der Lerngemeinschaft mit gleichen Pflichten zu sein.

... Lerninhalte $\mathrm{zu}$ präsentieren, so dass sie für die Schüler gut nachvollziehbar sind.

... Lerninhalte sorgfältig zu erläutern, so dass die Schüler sich diese gut merken können.

... Lehrinhalte zu präsentieren, so dass sie von den Schülern gut aufgenommen werden können.

... Lerninhalte sorgfältig und genau zu erklären.

... Lerninhalte für die Schüler gut nachvollziehbar zu referieren.

... Arbeitsschritte vorzuführen, damit die Schüler diese gut nachahmen können.

... den Schülern zu demonstrieren, wie man vorzugehen hat.

... Lerninhalte regelmäßig zu wiederholen, damit die Schüler sich diese gut einprägen können.
.23

.18

.54

.15

.16

$-.01$

$.19 \quad-.08$

.54

.24

.08

.52

.07

.33

.48

.23

.24

.46

$-.08$

.04

.15

.77

.01

$-.09 \quad .10$

.77

$-.09 \quad .03 \quad .08$

.73

$\begin{array}{lll}.04 & -.07 & .24\end{array}$

.70

$\begin{array}{lll}-.07 & .07 & .01\end{array}$

.66

.20

$-.10 \quad .10$

.54

.28

$\begin{array}{ll}-.07 & -.09\end{array}$

.50

.13

$.11 \quad .06$

.48 
... Vorgehensweisen zu zeigen, damit sich die Schüler

$\begin{array}{lll}.36 & -.06 \quad .08\end{array}$

.38 daran orientieren können.

... den Unterrichtsstoff regelmäßig zusammenzufassen,

.08

.30

.24

.30 damit die Schüler diese gut nachahmen können.

Gebildete Skalen

$\mathrm{P} \quad \mathrm{K}$

K $\quad \mathrm{SO}$

$\mathrm{T}$

Anzahl der Items ( $n)$

10

11

$10 \quad 10$

Cronbachs $\alpha$

.90

.90

.82

.84

.80

*Kursiv markiertes Item wurde ausgeschlossen, weil es die Reliabilität der Skala verschlechterte. 


\section{Anhang D: Mittelwerte (M) und Standardabweichungen (SD) der Items aus}

\section{der Studie 3}

Unterrichten bedeutet für mich...

... dazu beizutragen. dass sich die Schulklasse zu

einer Lerngemeinschaft entwickelt.

... das gemeinschaftliche Lernen unter den Schülern

zu fördern.

... zum Aufbau einer Lerngemeinschaft beizutragen.

... dass die Schüler gemeinsam lernen.

... dafür zu sorgen, dass die Schüler sich als

Mitglieder einer Lerngemeinschaft erfahren können.

... darauf zu achten, dass jeder Einzelne Teil einer

Lerngemeinschaft werden kann.

... das Gemeinschaftsgefühl der Klasse zu fördern.

... gegenseitigen Respekt und soziale Zugehörigkeit

unter den Schülerinnen zu unterstützen.

... eine angstfreie Lernatmosphäre zu schaffen, die

zum Erproben der eigenen Lernfähigkeiten

motiviert.

... die soziale Wertschätzung in der Gruppe der

Lernenden zu unterstützen.

... eine Lernumgebung zu schaffen, in der die

Schüler ihre eigenen Lernwege verfolgen können.

... eine Lernumgebung zu schaffen, die genügend

Raum für die eigenen Vorstellungen der Schüler lässt.

... die Schüler mit ihren eigenen Vermutungen auch eigene Lernwege gehen zu lassen.
SD

.76
.76 
... den Schülern Stoffe und Themen zur Auswahl zu geben.

... die Schüler mit ihren eigenen alltäglichen

Erfahrungen Neues entdecken zu lassen.

... auch Fehler der Schüler zu akzeptieren und sie

weitermachen zu lassen, bis sie selbst gemerkt haben, dass etwas nicht stimmt.

... die Schüler mit ihren eigenen Vermutungen auch einmal in die Irre gehen zu lassen, bis sie es selbst gemerkt haben.

... bei der Stoffauswahl auch auf individuelle

Wünsche der Schüler einzugehen.

... bei der Themenauswahl die Interessen der

Schüler einzubeziehen.

... die Schüler selbst darüber bestimmen zu lassen,

mit welchen Themen sie sich beschäftigen möchten.

... Schülerantworten erst einmal zu akzeptieren und sie selbst weiter entdecken zu lassen.

... mit den Schülern zu reden, wenn ihnen etwas nicht gefällt.

... mir auch Zeit für die Schüler zu nehmen, wenn sie etwas mit mir bereden wollen.

... mich auch um die Probleme der Schüler zu

kümmern.

... die Wünsche der Schüler soweit wie möglich zu erfüllen.

... den Schülern zu helfen wie ein Freund. 
... dass ich zusammen mit meinen Schülern ein

gemeinsames Ziel verfolge.

... für Lernsituationen zu sorgen, in denen sich die

Schüler kompetent erleben.

... obwohl Experte, ein Mitglied der

Lerngemeinschaft mit gleichen Orientierungen zu sein.

... obwohl Experte, ein Mitglied der

Lerngemeinschaft mit gleichen Pflichten zu sein.

... Lerninhalte zu präsentieren, so dass sie für die

Schüler gut nachvollziehbar sind.

... Lerninhalte sorgfältig zu erläutern, so dass die

Schüler sich diese gut merken können.

... Lehrinhalte zu präsentieren, so dass sie von den

Schülern gut aufgenommen werden können.

... Lerninhalte sorgfältig und genau zu erklären.

... Lerninhalte für die Schüler gut nachvollziehbar zu referieren.

... Arbeitsschritte vorzuführen, damit die Schüler diese gut nachahmen können.

zusammenzufassen, damit die Schüler diese gut nachahmen können. 


\section{Eidesstattliche Erklärung}

Ich versichere, dass ich die eingereichte Dissertation „Lehrerüberzeugungen zum Lehren und Lernen“" selbstständig und ohne unerlaubte Hilfsmittel verfasst habe. Anderer als der von mir angegebenen Hilfsmittel und Schriften habe ich mich nicht bedient. Alle wörtlich oder sinngemäß den Schriften anderer Autoren entnommenen Stellen habe ich kenntlich gemacht.

Datum

Unterschrift 